Supporting Information

for

\title{
Redox Cyclization of Amides and Sulfonamides with Nitrous Oxide for Direct Synthesis of Heterocycles
}

Zhencheng Lai, Chaorong Wang, Jiaming Li, and Sunliang Cui* Institute of Drug Discovery and Design, College of Pharmaceutical Sciences, Zhejiang University, Hangzhou 310058, China

E-mail: slcui@zju.edu.cn

\section{Contents}

1 General Information

S2-S3

2 Typical Procedure for Synthesis of 3a

S4

3 Optimization and Typical Synthesis of 5a

S5-S6

4 Synthesis of 6

S6-S7

5 Typical Procedure for Synthesis of 7a

S7

6 Control Reaction

$7 \quad$ Characterization of Compounds

$\mathrm{S} 8-\mathrm{S} 30$

8 X-ray Crystallographic Data

S30-S32

9 References

S32

10 Copies of NMR Spectra

S33-S116 


\section{General Information}

The reaction temperatures are reported corresponding to the oil bath temperature. Reactions were monitored by thin layer chromatography (TLC) using silicycle pre-coated silica gel plates. Column chromatography was performed over silica gel (200-300mesh).

Melting points were measured with X-4 micro melting point apparatus. HRMS were performed on Agilent Technologies 6224 TOF LC/MS apparatus (ESI). ${ }^{1} \mathrm{H}$ NMR spectra and ${ }^{13} \mathrm{C}$ NMR spectra were recorded on a Bruker AV-600 spectrometer, Bruker AV-500 spectrometer or a WNMR-I-400 spectrometer in $\mathrm{CD}_{3} \mathrm{Cl}$ or DMSO-d 6 (contain internal TMS). Chemical shifts of ${ }^{1} \mathrm{H}$ NMR spectra were reported in ppm with the internal TMS signal at 0 ppm as a standard, and chemical shifts of ${ }^{13} \mathrm{C}$ NMR spectra were reported in ppm with the chloroform signal at $77.16 \mathrm{ppm}$ as a standard. The data is being reported as $(\mathrm{s}=$ singlet, $\mathrm{d}=$ doublet, $\mathrm{t}=$ triplet, $\mathrm{q}=$ quartet, $\mathrm{dd}=$ double doublet, $\mathrm{dt}=$ double of triplet, $\mathrm{m}=$ multiplet or unresolved, $\mathrm{br}=$ broad singlet, coupling constant(s) in Hz, integration).

Solvents and $N, N, N^{\prime}, N^{\prime}$-tetramethylethylenediamine (TMEDA) were obtained commercially and dried with sodium and $\mathrm{CaH}_{2}$ respectively. Potassium 2-methyl-2-butoxide was obtained commercially .

All starting material of amides and sulfonamides were shown in Figure S1 and Figure S2. 1a was synthesized according to the reported methods. ${ }^{1} \mathbf{1 b}-\mathbf{1 e}, \mathbf{1 h}-\mathbf{1 j}$, 11, 1m, 1o - 1r, 1z were prepared from commercially available benzoic acids and tert-butylamine according to the reported methods. ${ }^{2} \mathbf{1 s}-\mathbf{1 x}$ could be easily synthesized by benzoyl chloride and commercially available amines. ${ }^{3,4} \mathbf{1 y}$ was prepared by the reported methods. ${ }^{5}$

$\mathbf{4 a}-\mathbf{4 k}$ were synthesized according to the reported methods. ${ }^{6}$ 
<smiles>O=C(NOCCOCCOCCO)c1ccccc1</smiles>

$1 a$<smiles>CC(C)(C)NC(=O)c1ccccc1</smiles>

1b<smiles>CCCCNC(=O)c1ccc(C2=CCCC2)cc1</smiles><smiles>CC(C)(C)NC(=O)c1ccc(C#Cc2ccc(C(=O)NC(=O)c3ccc(C(=O)NC(C)(C)C)cc3)cc2)cc1</smiles><smiles>CCC(C)NC(=O)c1ccc(C(=O)NC(C)(C)C)cc1</smiles><smiles>CN(C)c1ccc(C(=O)Nc2ccc(C(=O)NC(C)(C)C)cc2)cc1</smiles><smiles>COc1ccccc1C(=O)NC(C)(C)C</smiles><smiles>CC(C)(C)NC(=O)c1ccc2ccccc2c1</smiles><smiles>CC(C)(C)NC(=O)c1ccncc1</smiles>

19<smiles>O=C(NC1CC1)c1ccccc1</smiles>

1u<smiles>Cc1ccc(C(=O)NC(C)(C)C)s1</smiles>

$1 \mathrm{r}$<smiles>CC(C)(NC(=O)c1ccccc1)c1ccccc1</smiles>

1v<smiles>O=C(Nc1ccccc1)c1ccccc1</smiles>

$1 \mathrm{~s}$<smiles>CC(C)NC(=O)c1ccccc1</smiles>

$1 \mathrm{t}$<smiles>CONC(=O)c1ccccc1</smiles>

$1 \mathrm{y}$<smiles>[Y7]c1cccc(F)c1C(=O)N[14C](=O)c1ccccc1</smiles>

Figure S1. Scope of Amides<smiles>CC(C)(C)NS(=O)(=O)c1ccccc1</smiles>

$4 a$<smiles>CC(C)(NS(=O)(=O)c1ccccc1)c1ccccc1</smiles>

$4 e$<smiles>CNS(=O)(=O)c1ccccc1</smiles>

4b<smiles>CC(C)NS(=O)(=O)c1ccccc1</smiles>

$4 c$<smiles>COc1ccc(NC(=O)c2ccccc2)cc1</smiles><smiles>CC(NS(=O)(=O)c1ccccc1)c1ccccc1</smiles>

$4 f$<smiles>Cc1ccc(S(=O)(=O)NC(C)(C)C)cc1</smiles>

$4 \mathrm{~g}$<smiles>O=S(=O)(NC1CC1)c1ccccc1</smiles>

4d<smiles>CC(C)(C)NS(=O)(=O)c1ccc(C(C)(C)C)cc1</smiles><smiles>COc1ccc(S(=O)(=O)NC(C)(C)C)cc1</smiles>

Figure S2. Scope of Sulfonamides 


\section{Typical Procedure for Synthesis of 3a}

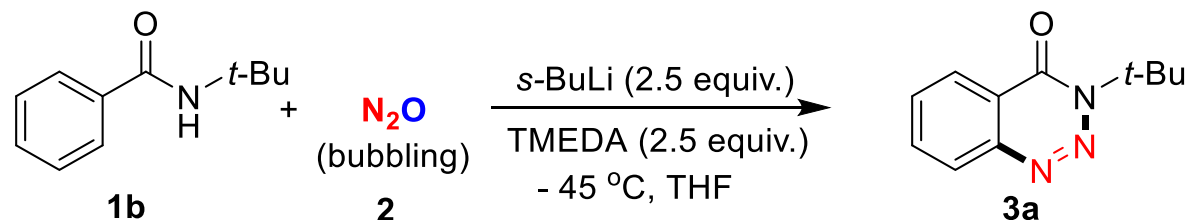

To a stirred solution of $N$-tert-butyl benzamide $\mathbf{1 b}(88 \mathrm{mg}, 0.5 \mathrm{mmol})$ and TMEDA (145 mg, $1.25 \mathrm{mmol})$ in dry THF $(3 \mathrm{~mL})$ at $-45^{\circ} \mathrm{C}$ under Argon was added dropwise sec-butyllithium (1.3 $\mathrm{M}$ in hexane, $0.97 \mathrm{~mL}, 1.25 \mathrm{mmol})$. After stirring at $-45^{\circ} \mathrm{C}$ for $2 \mathrm{hrs}, \mathrm{N}_{2} \mathrm{O} 2$ was bubbled to the cold $\left(-45^{\circ} \mathrm{C}\right)$ solution for $1 \mathrm{hr}$. Afterwards, the reaction mixture was quenched by the addition of saturated aqueous $\mathrm{NH}_{4} \mathrm{Cl}$, and the aqueous layer was extracted with EA (three times). The combined organic layer was dried over $\mathrm{Na}_{2} \mathrm{SO}_{4}$ and concentrated. The crude product was purified by column chromatography on silica gel eluted with PE/EA (v/v, 20:1) to give compound 3a (86 $\mathrm{mg}, 85 \%$ yield) as pale yellow solid.

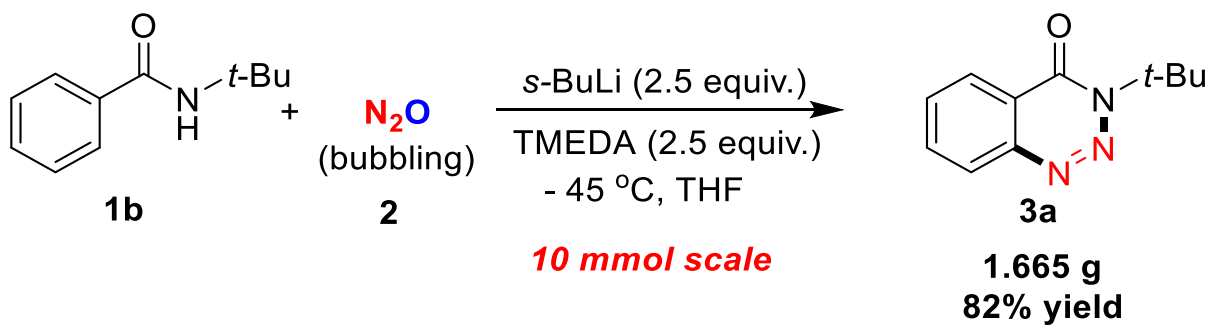

A three-necked round bottom flask equipped with magnetic stirrer bar was charged with $N$-tert-butyl benzamide $1 \mathrm{a}(1.77 \mathrm{~g}, 10 \mathrm{mmol})$, then evacuated and purged with Argon three times. Afterwards, flask was added a solution of TMEDA (2.9 g, $25 \mathrm{mmol})$ in dry THF (60 mL) via syringe. sec-butyllithium (1.3 $\mathrm{M}$ in hexane, $19.23 \mathrm{~mL}, 25 \mathrm{mmol}$ ) was added dropwise to the above mixture at $-45^{\circ} \mathrm{C}$, and stirred for 2 hrs. After bubbling $\mathrm{N}_{2} \mathrm{O} 2$ to the cold $\left(-45^{\circ} \mathrm{C}\right)$ solution for $1 \mathrm{hrs}$, the reaction mixture was quenched by the addition of saturated aqueous $\mathrm{NH}_{4} \mathrm{Cl}$, and the aqueous layer was extracted with EA (three times). The combined organic layer was dried over $\mathrm{Na}_{2} \mathrm{SO}_{4}$ and concentrated. The crude product was purified by column chromatography on silica gel eluted with PE/EA (v/v, 20:1) to give compound 3a (1.665 g, 82\% yield) as pale yellow solid. 


\section{Optimization and Typical Synthesis of 5a}

All optimization reactions were carried out on $0.5 \mathrm{mmol}$ scale. RLi and additive were used in $2 \mathrm{mmol}$ (4.0 equiv.). Yield refers to isolated product by column chromatography on silica gel eluted with PE/EA (v/v, 20:1).<smiles>O=S(=O)(N[131I])c1ccccc1</smiles>

$4 a$

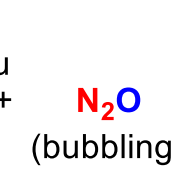

2

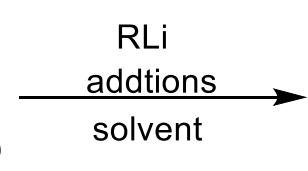

solvent<smiles>CC(C)(C)N1N=Nc2ccccc2S1(=O)=O</smiles>

$5 a$

\begin{tabular}{|c|c|c|c|c|c|}
\hline entry & RLi & additive & $\begin{array}{l}\text { temp. } \\
\left({ }^{\circ} \mathrm{C}\right)\end{array}$ & solvent & $\begin{array}{l}\text { yield } \\
(\%)\end{array}$ \\
\hline 1 & $s$-BuLi & TMEDA & -45 & THF & 18 \\
\hline 2 & $s$-BuLi & TMEDA & 0 & THF & $<10$ \\
\hline 3 & $s$-BuLi & TMEDA & 25 & THF & 0 \\
\hline 4 & $s$-BuLi & DMPU & -45 & THF & $<10$ \\
\hline 5 & n-BuLi & TMEDA & -45 & THF & $<10$ \\
\hline 6 & LDA & none & 0 & THF & 0 \\
\hline 7 & $n$-BuLi & $t$-BuOK & -78 & THF & 42 \\
\hline 8 & n-BuLi & $\mathrm{KOC}(\mathrm{Et}) \mathrm{Me}_{2}$ & -78 & THF & 48 \\
\hline 9 & n-BuLi & $t-\mathrm{BuOK}$ & -45 & THF & 21 \\
\hline 10 & $\begin{array}{l}n-\mathrm{BuLi} \\
(2.5 \mathrm{eq})\end{array}$ & $\begin{array}{c}\mathrm{KOC}(\mathrm{Et}) \mathrm{Me}_{2} \\
(2.5 \mathrm{eq})\end{array}$ & -78 & THF & 40 \\
\hline 11 & $\begin{array}{l}n-\mathrm{BuLi} \\
(6.0 \mathrm{eq})\end{array}$ & $\begin{array}{c}\mathrm{KOC}(\mathrm{Et}) \mathrm{Me}_{2} \\
\quad(6.0 \mathrm{eq})\end{array}$ & -78 & THF & 47 \\
\hline 12 & n-BuLi & $\mathrm{KOC}(\mathrm{Et}) \mathrm{Me}_{2}$ & -78 & MTBE & 53 \\
\hline 13 & n-BuLi & $\mathrm{KOC}(\mathrm{Et}) \mathrm{Me}_{2}$ & -78 & $\mathrm{Et}_{2} \mathrm{O}$ & 32 \\
\hline 14 & n-BuLi & $\mathrm{KOC}(\mathrm{Et}) \mathrm{Me}_{2}$ & -78 & 2-MeTHF & 68 \\
\hline
\end{tabular}

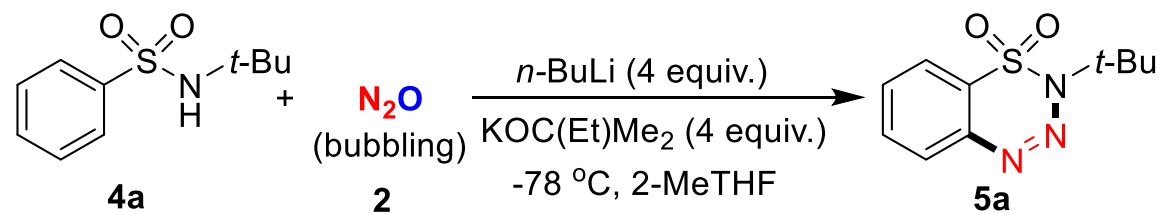

An oven-dried Schlenk tube equipped with magnetic stirrer bar was charged with $\mathrm{KOC}(\mathrm{Et}) \mathrm{Me}_{2}(252 \mathrm{mg}, 2 \mathrm{mmol})$, then evacuated and purged with argon three times. Then $1 \mathrm{~mL}$ anhydrous hexane was added by a syringe, and $n$-BuLi $(2.5 \mathrm{M}$ in hexane, $0.8 \mathrm{~mL}, 2.0 \mathrm{mmol}$ ) was added dropwise at room temperature and stirred for 30 mins.

To a stirred $-78{ }^{\circ} \mathrm{C}$ solution of $N$-(tert-butyl)benzenesulfonamide $4 \mathbf{a}$ (107 $\mathrm{mg}, 0.5$ 
mmol) in dry 2-MeTHF ( $1 \mathrm{~mL})$ under Argon was added dropwise the above mixture of $\mathrm{KOC}(\mathrm{Et}) \mathrm{Me}_{2}$ and $n$-BuLi. After stirring at $-78{ }^{\circ} \mathrm{C}$ for $1 \mathrm{hr}, \mathrm{N}_{2} \mathrm{O} 2$ was bubbled to the solution for $1 \mathrm{hr}$ at this temperature. Afterward, the reaction mixture was quenched by saturated aqueous $\mathrm{NH}_{4} \mathrm{Cl}$, and the aqueous layer was extracted with $\mathrm{EA}$ (three times). The combined organic layer was dried over $\mathrm{Na}_{2} \mathrm{SO}_{4}$ and concentrated. The crude product was purified by column chromatography on silica gel eluted with PE/EA (v/v, $20: 1)$ to give compound $\mathbf{5 a}(81 \mathrm{mg}, 68 \%$ yield $)$ as pale orange solid.

\section{Synthesis of 6}

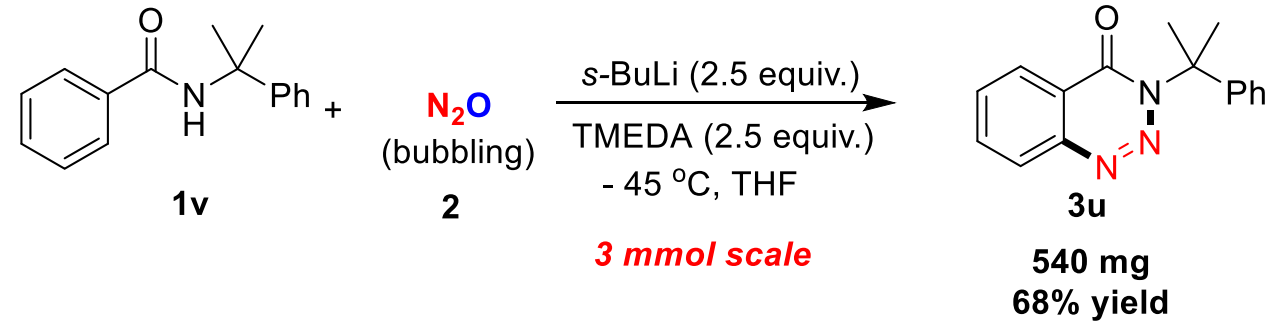

\section{Procedure for the synthesis of $3 u$}

A three-necked round bottom flask equipped with magnetic stirrer bar was charged with $1 \mathbf{u}(717 \mathrm{mg}, 3 \mathrm{mmol})$, then evacuated and purged with Argon three times. Afterwards, the flask was added a solution of TMEDA (879 $\mathrm{mg}, 7.5 \mathrm{mmol}$ ) in dry THF (18 mL) via syringe. sec-butyllithium (1.3M in hexane, $5.77 \mathrm{~mL}, 7.5 \mathrm{mmol})$ was added dropwise to the above mixture at $-45{ }^{\circ} \mathrm{C}$, and stirred for $2 \mathrm{hrs}$. After bubbling $\mathrm{N}_{2} \mathrm{O} 2$ to the coldsolution for $1 \mathrm{hr}$ at $-45{ }^{\circ} \mathrm{C}$, the reaction mixture was quenched by saturated aqueous $\mathrm{NH}_{4} \mathrm{Cl}$, and the aqueous layer was extracted with $\mathrm{EA}$ (three times). The combined organic layer was dried over $\mathrm{Na}_{2} \mathrm{SO}_{4}$ and concentrated. The crude product was purified by column chromatography on silica gel eluted with PE/EA (v/v, 20:1 to $10: 1$ ) to give compound $3 \mathbf{u}$ (540 $\mathrm{mg}, 68 \%$ yield) as pale yellow solid.

\section{Procedure for the synthesis of 6}<smiles>CC(C)(c1ccccc1)n1nnc2ccccc2c1=O</smiles>

3u

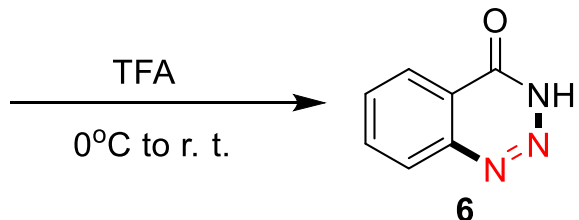

6 
A round-bottom flask equipped with magnetic stirrer bar was charged with $\mathbf{3 u}$ $(530 \mathrm{mg}, 2 \mathrm{mmol})$ and TFA $(2 \mathrm{~mL})$ at $0{ }^{\circ} \mathrm{C}$. The mixture was allowed to stand at room temperature overnight. After completion which was detected by TLC, the reaction was quenched by the saturated aqueous $\mathrm{NaHCO}_{3}$. Afterwards, the aqueous layer was extracted with EA (five times), and the combined organic layer was dried over $\mathrm{Na}_{2} \mathrm{SO}_{4}$ and concentrated. The crude product was purified by column chromatography on silica gel eluted with PE/EA (v/v, 4:1) to give compound 6 (241 mg, 82\% yield) as brown solid.

\section{Typical Procedure for the Synthesis of 7a}

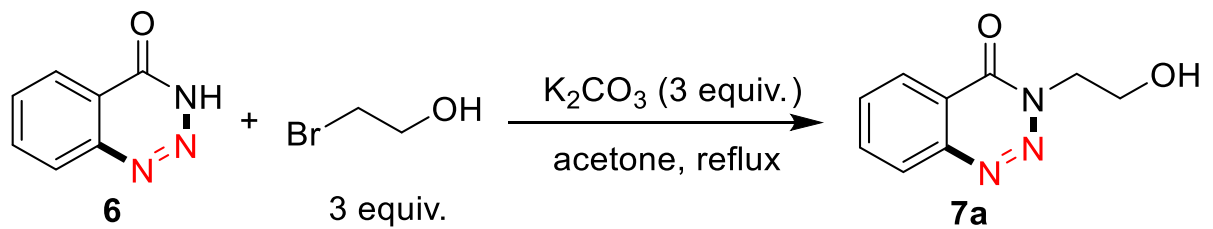

In a Schlenk tube was charged with $6(29 \mathrm{mg}, 0.2 \mathrm{mmol})$ and acetone $(1 \mathrm{~mL})$. The mixture was stirred at reflux for $14 \mathrm{~h}$. Afterward, the reaction solvent was evaporated under reduced pressure. The crude product was purified by column chromatography on silica gel eluted with $\mathrm{PE} / \mathrm{EA}(\mathrm{v} / \mathrm{v}=4: 1)$ to give compound $7 \mathbf{a}$ (36 $\mathrm{mg}, 95 \%$ yield) as white solid.

\section{Control Reaction}<smiles></smiles>

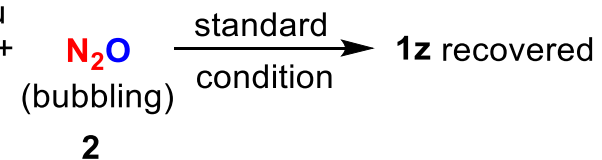

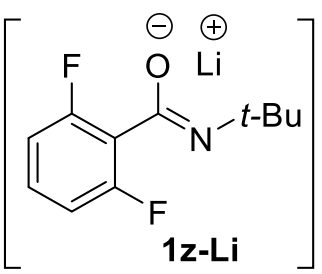

To a stirred solution of $N$-tert-butyl benzamide $\mathbf{1 z}(106 \mathrm{mg}, 0.5 \mathrm{mmol})$ and TMEDA (145 mg, $1.25 \mathrm{mmol})$ in dry THF $(3 \mathrm{~mL})$ at $-45{ }^{\circ} \mathrm{C}$ under Argon was added dropwise sec-butyllithium (1.3M in hexane, $0.97 \mathrm{~mL}, 1.25 \mathrm{mmol})$. After stirring at -45 ${ }^{\circ} \mathrm{C}$ for $2 \mathrm{hrs}, \mathrm{N}_{2} \mathrm{O} 2$ was bubbled to the cold solution for $1 \mathrm{hr}$ at $-45{ }^{\circ} \mathrm{C}$. Afterwards, the reaction mixture was quenched by saturated aqueous $\mathrm{NH}_{4} \mathrm{Cl}$, and the aqueous layer was extracted with EA (three times). The combined organic layer was dried over 
$\mathrm{Na}_{2} \mathrm{SO}_{4}$ and concentrated. Only $\mathbf{1 z}$ was observed in the residue and isolated in quantitative recovery.<smiles>COc1ccc(/N=N/c2ccc(OC)cc2)cc1</smiles>

To a stirred solution of 4-bromoanisole $(187 \mathrm{mg}, 1.0 \mathrm{mmol})$ in dry THF $(3 \mathrm{~mL})$ at $-45{ }^{\circ} \mathrm{C}$ under Argon was added dropwise $n$-BuLi (2.5 M in hexane, $0.48 \mathrm{~mL}, 1.2$ mmol). After stirring for 30 mins, $\mathrm{N}_{2} \mathrm{O} 2$ was bubbled to the solution for $1 \mathrm{hr}$ at $-45^{\circ} \mathrm{C}$. Afterwards, the reaction mixture was quenched by saturated aqueous $\mathrm{NH}_{4} \mathrm{Cl}$, and the aqueous layer was extracted with EA (three times). The combined organic layer was dried over $\mathrm{Na}_{2} \mathrm{SO}_{4}$ and concentrated. The crude product was purified by column chromatography on silica gel eluted with PE/EA (v/v, 30:1 to 20:1) to give compound 9 (131 mg, 55\% yield) as yellow solid.

\section{Characterization of Compounds}<smiles>CC(C)(C)n1nnc2ccccc2c1=O</smiles>

\section{3-(tert-butyl)benzo $[d][1,2,3]$ triazin-4(3H)-one}

3a: Pale yellow solid, m. p. $59.5-63.1{ }^{\circ} \mathrm{C}(86 \mathrm{mg}, 85 \%$ yield on $0.5 \mathrm{mmol}$ scale; $82 \%$ yield on $10 \mathrm{mmol}$ scale)

TLC: $\mathrm{R}_{\mathrm{f}}=0.3($ Petroleum ether/EtOAc $=20 / 1)$

${ }^{1}$ H NMR (500 MHz, CDCl $) \delta 8.32\left(\mathrm{dd}, J_{1}=8.0 \mathrm{~Hz}, J_{2}=1.0 \mathrm{~Hz}, 1 \mathrm{H}\right), 8.08(\mathrm{~d}, \boldsymbol{J}$ $=8.5 \mathrm{~Hz}, 1 \mathrm{H}), 7.92-7.87(\mathrm{~m}, 1 \mathrm{H}), 7.76-7.72(\mathrm{~m}, 1 \mathrm{H}), 1.80(\mathrm{~s}, 9 \mathrm{H})$.

${ }^{13}$ C NMR (100 MHz, CDCl 3$) \delta 156.1,143.7,134.4,131.8,127.5,124.9,120.7$, $64.9,28.5$. 
HRMS (ESI-TOF) m/z: $[\mathrm{M}+\mathrm{Na}]^{+}$Calcd for $\mathrm{C}_{11} \mathrm{H}_{13} \mathrm{~N}_{3} \mathrm{ONa}$ 226.0956; Found 226.0972 .

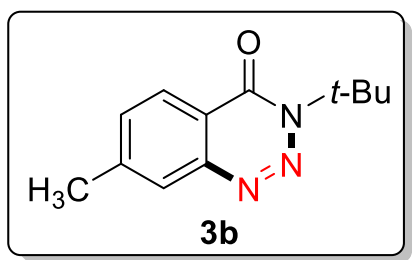

\section{3-(tert-butyl)-7-methylbenzo $[d][1,2,3]$ triazin-4(3H)-one}

3b: Pale yellow solid, m. p. $75.1-78.3{ }^{\circ} \mathrm{C}(96 \mathrm{mg}, 89 \%$ yield on $0.5 \mathrm{mmol}$ scale $)$

TLC: $\mathrm{R}_{\mathrm{f}}=0.3$ (Petroleum ether $\left./ \mathrm{EtOAc}=20 / 1\right)$

${ }^{1}$ H NMR (400 MHz, CDCl $) \delta 8.23(\mathrm{~d}, J=8.0 \mathrm{~Hz}, 1 \mathrm{H}), 7.89(\mathrm{~s}, 1 \mathrm{H}), 7.58(\mathrm{~d}, J$ $=8.0 \mathrm{~Hz}, 1 \mathrm{H}), 2.60(\mathrm{~s}, 3 \mathrm{H}), 1.83(\mathrm{~s}, 9 \mathrm{H})$.

${ }^{13}$ C NMR (100 MHz, CDCl 3 ) $\delta$ 156.2, 145.7, 143.9, 133.4, 127.0, 124.8, 118.4, 64.9, 28.6, 21.9.

HRMS (ESI-TOF) m/z: $[\mathrm{M}+\mathrm{Na}]^{+}$Calcd for $\mathrm{C}_{12} \mathrm{H}_{15} \mathrm{~N}_{3} \mathrm{ONa}$ 240.1113; Found 240.1124 .

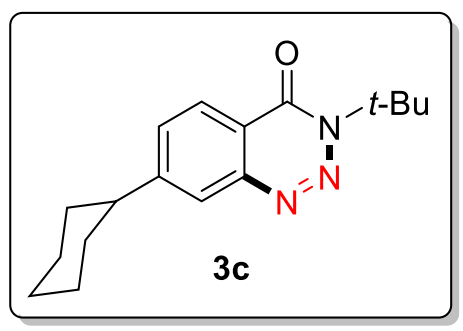

3-(tert-butyl)-7-cyclohexylbenzo $[d][1,2,3]$ triazin-4(3H)-one

3c: Pale yellow solid, m. p. $145.8-148.6^{\circ} \mathrm{C}(63 \mathrm{mg}, 44 \%$ yield on $0.5 \mathrm{mmol}$ scale $)$

TLC: $\mathrm{R}_{\mathrm{f}}=0.2($ Petroleum ether $/$ EtOAc $=20 / 1)$

${ }^{1} \mathbf{H}$ NMR $\left(600 \mathrm{MHz}, \mathbf{C D C l}_{3}\right) \delta 8.23(\mathrm{~d}, J=8.4 \mathrm{~Hz}, 1 \mathrm{H}), 7.89(\mathrm{~d}, J=1.2 \mathrm{~Hz}, 1 \mathrm{H})$, $7.61\left(\mathrm{dd}, J_{1}=8.4 \mathrm{~Hz}, J_{2}=1.2 \mathrm{~Hz}, 1 \mathrm{H}\right), 2.76-2.70(\mathrm{~m}, 1 \mathrm{H}), 1.97-1.88(\mathrm{~m}, 4 \mathrm{H}), 1.80$ (s, 9H), $1.55-1.28(\mathrm{~m}, 6 \mathrm{H})$. 
${ }^{13}$ C NMR (100 MHz, $\left.\mathbf{C D C l}_{3}\right) \delta$ 156.3, 155.6, 144.2, 131.6, 124.9, 124.9, 118.7, $64.8,44.8,34.1,28.6,26.7,25.9$.

HRMS (ESI-TOF) m/z: $[\mathrm{M}+\mathrm{Na}]^{+}$Calcd for $\mathrm{C}_{17} \mathrm{H}_{23} \mathrm{~N}_{3} \mathrm{ONa}$ : 308.1739; Found 308.1747 .

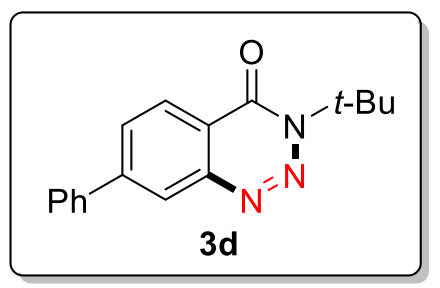

3-(tert-butyl)-7-phenylbenzo $[d][1,2,3]$ triazin-4(3H)-one

3d: Pale yellow solid, m. p. $81.3-82.7^{\circ} \mathrm{C}(85 \mathrm{mg}, 61 \%$ yield on $0.5 \mathrm{mmol}$ scale $)$

TLC: $\mathrm{R}_{\mathrm{f}}=0.4$ (Petroleum ether $\left./ \mathrm{EtOAc}=20 / 1\right)$

${ }^{1} \mathbf{H}$ NMR (600 MHz, CDCl 3$) \delta 8.35(\mathrm{~d}, J=8.4 \mathrm{~Hz}, 1 \mathrm{H}), 8.25(\mathrm{~s}, 1 \mathrm{H}), 7.95(\mathrm{~d}, J$ $=8.4 \mathrm{~Hz}, 1 \mathrm{H}), 7.69(\mathrm{~d}, J=7.8 \mathrm{~Hz}, 2 \mathrm{H}), 7.50(\mathrm{t}, J=7.4 \mathrm{~Hz}, 2 \mathrm{H}), 7.44(\mathrm{t}, J=7.2 \mathrm{~Hz}$, $1 \mathrm{H}), 1.82(\mathrm{~s}, 9 \mathrm{H})$.

${ }^{13}$ C NMR (100 MHz, $\left.\mathbf{C D C l}_{3}\right) \delta 156.0,147.5,144.3,138.8,130.8,129.2,128.9$, 127.5, 125.6, 125.3, 119.4, 65.0, 28.6.

HRMS (ESI-TOF) m/z: $[\mathrm{M}+\mathrm{Na}]^{+}$Calcd for $\mathrm{C}_{17} \mathrm{H}_{17} \mathrm{~N}_{3} \mathrm{ONa}$ 302.1269; Found 302.1271 .

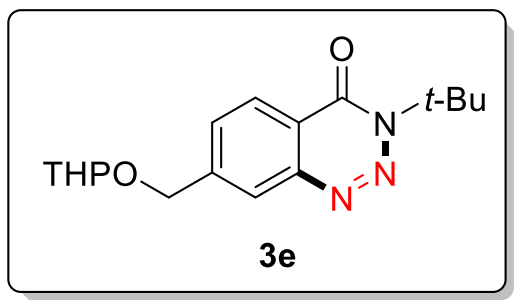

3-(tert-butyl)-7-(((tetrahydro-2H-pyran-2-l)oxy)methyl)benzo[d][1,2,3] triazin-4(3H)-one

3e: Yellow oil, (64 mg, 40\% yield on $0.5 \mathrm{mmol} \mathrm{scale)}$

TLC: $\mathrm{R}_{\mathrm{f}}=0.3($ Petroleum ether $/ \mathrm{EtOAc}=10 / 1)$ 
${ }^{1} \mathbf{H}$ NMR (400 MHz, CDCl $) \delta 8.27(\mathrm{~d}, J=8.0 \mathrm{~Hz}, 1 \mathrm{H}), 8.06(\mathrm{~d}, J=1.2 \mathrm{~Hz}, 1 \mathrm{H})$, $7.71\left(\mathrm{dd}, J_{1}=8.4, J_{2}=1.6 \mathrm{~Hz}, 1 \mathrm{H}\right), 4.96(\mathrm{~d}, J=13.6 \mathrm{~Hz}, 1 \mathrm{H}), 4.78-4.65(\mathrm{~m}, 2 \mathrm{H})$, $3.92-3.80(\mathrm{~m}, 1 \mathrm{H}), 3.58-3.50(\mathrm{~m}, 1 \mathrm{H}), 1.89-1.86(\mathrm{~m}, 1 \mathrm{H}), 1.79(\mathrm{~s}, 9 \mathrm{H}), 1.77-$ $1.65(\mathrm{~m}, 2 \mathrm{H}), 1.64-1.49(\mathrm{~m}, 3 \mathrm{H})$.

${ }^{13}$ C NMR (100 MHz, $\left.\mathbf{C D C l}_{\mathbf{3}}\right) \delta$ 156.1, 146.2, 143.9, 130.8, 125.4, 125.2, 119.8, $98.3,67.8,65.0,62.3,30.5,28.6,25.4,19.3$.

HRMS (ESI-TOF) m/z: $[\mathrm{M}+\mathrm{Na}]^{+}$Calcd for $\mathrm{C}_{17} \mathrm{H}_{23} \mathrm{~N}_{3} \mathrm{O}_{3} \mathrm{Na}$ 340.1637; Found 340.1648 .

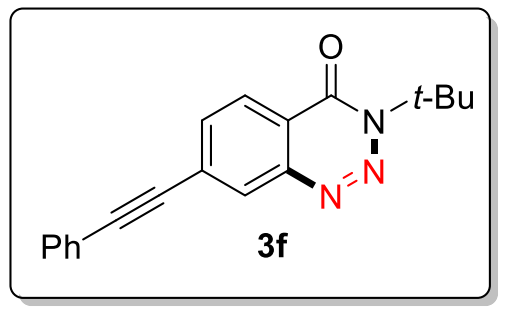

\section{3-(tert-butyl)-7-(phenylethynyl)benzo $[d][1,2,3]$ triazin-4(3H)-one}

3f: Orange solid, m. p. $91.9-94.8{ }^{\circ} \mathrm{C}(82 \mathrm{mg}, 54 \%$ yield on $0.5 \mathrm{mmol}$ scale $)$

TLC: $R_{\mathrm{f}}=0.5($ Petroleum ether $/$ EtOAc $=10 / 1)$

${ }^{1}$ H NMR (400 MHz, CDCl $) \delta 8.29(\mathrm{~d}, J=8.0 \mathrm{~Hz}, 1 \mathrm{H}), 8.22(\mathrm{~d}, J=1.2 \mathrm{~Hz}, 1 \mathrm{H})$, $7.83\left(\mathrm{dd}, J_{1}=8.4, J_{2}=1.6 \mathrm{~Hz}, 1 \mathrm{H}\right), 7.63-7.56(\mathrm{~m}, 2 \mathrm{H}), 7.43-7.37(\mathrm{~m}, 3 \mathrm{H}), 1.81(\mathrm{~s}$, 9H).

${ }^{13}$ C NMR (100 MHz, CDCl $) \delta 155.7,143.7,134.5,131.9,130.2,129.9,129.3$, 128.6, 125.2, 122.1, 119.8, 94.0, 87.6, 65.3, 28.5.

HRMS (ESI-TOF) m/z: $[\mathrm{M}+\mathrm{Na}]^{+}$Calcd for $\mathrm{C}_{19} \mathrm{H}_{17} \mathrm{~N}_{3} \mathrm{ONa} 326.1269$; Found 326.1274 .

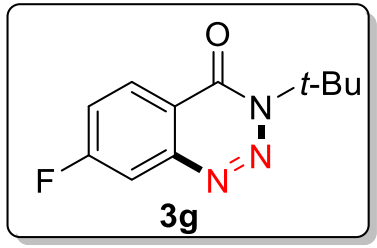

3-(tert-butyl)-7-fluorobenzo[d][1,2,3]triazin-4(3H)-one 
3g: Pale orange solid, m. p. $77.4-80.5^{\circ} \mathrm{C}(70 \mathrm{mg}, 64 \%$ yield on $0.5 \mathrm{mmol}$ scale)

TLC: $\mathrm{R}_{\mathrm{f}}=0.3($ Petroleum ether $/$ EtOAc $=20 / 1)$

${ }^{1}$ H NMR (500 MHz, CDCl $) \delta 8.29\left(\mathrm{dd}, J_{1}=9.0 \mathrm{~Hz}, J_{2}=5.5 \mathrm{~Hz}, 1 \mathrm{H}\right), 7.65(\mathrm{dd}$, $\left.J_{1}=8.4 \mathrm{~Hz}, J_{2}=2.5 \mathrm{~Hz}, 1 \mathrm{H}\right), 7.37-7.41(\mathrm{~m}, 1 \mathrm{H}), 1.74(\mathrm{~s}, 9 \mathrm{H})$.

${ }^{13}$ C NMR (125 MHz, CDCl3) $\delta$ 166.2, 164.2, 154.5, 144.7, 144.6, 119.9, 119.8, 116.7, 116.7, 111.8, 111.7, 64.5, 27.6.

HRMS (ESI-TOF) m/z: $[\mathrm{M}+\mathrm{Na}]^{+}$Calcd for $\mathrm{C}_{11} \mathrm{H}_{12} \mathrm{FN}_{3} \mathrm{ONa}$ 244.0862; Found 244.0872 .

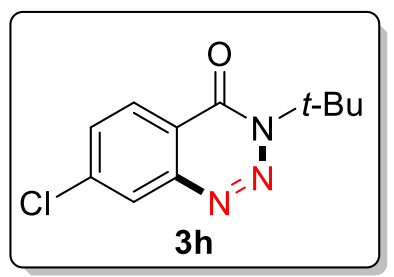

\section{3-(tert-butyl)-7-chlorobenzo[d][1,2,3]triazin-4(3H)-one}

3h: Pale orange solid, m. p. $82.0-83.2{ }^{\circ} \mathrm{C}(49 \mathrm{mg}, 42 \%$ yield on $0.5 \mathrm{mmol}$ scale $)$

TLC: $\mathrm{R}_{\mathrm{f}}=0.3($ Petroleum ether $/$ EtOAc $=20 / 1)$

${ }^{1}$ H NMR (500 MHz, CDCl 3$) \delta 8.26(\mathrm{~d}, J=8.5 \mathrm{~Hz}, 1 \mathrm{H}), 8.06(\mathrm{~d}, J=2.0 \mathrm{~Hz}, 1 \mathrm{H})$, $7.68\left(\mathrm{dd}, J_{1}=8.5 \mathrm{~Hz}, J_{2}=2.0 \mathrm{~Hz}, 1 \mathrm{H}\right), 1.79(\mathrm{~s}, 9 \mathrm{H})$.

${ }^{13}$ C NMR (125 MHz, $\left.\mathbf{C D C l}_{3}\right) \delta 155.5,144.4,140.8,132.4,126.9,126.8,119.16$, $65.5,28.5$.

HRMS (ESI-TOF) m/z: $[\mathrm{M}+\mathrm{Na}]^{+}$Calcd for $\mathrm{C}_{11} \mathrm{H}_{12} \mathrm{ClN}_{3} \mathrm{ONa} 260.0567$; Found 260.0569 .

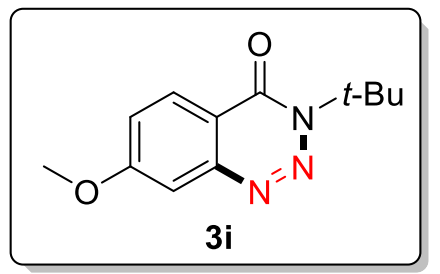

3-(tert-butyl)-7-methoxybenzo[d][1,2,3]triazin-4(3H)-one 
3i: White solid, m. p. $117.8-119.6{ }^{\circ} \mathrm{C}(80 \mathrm{mg}, 68 \%$ yield on $0.5 \mathrm{mmol}$ scale $)$

TLC: $\mathrm{R}_{\mathrm{f}}=0.4($ Petroleum ether $/$ EtOAc $=10 / 1)$

${ }^{1}$ H NMR (400 MHz, CDCl $) \delta 8.24(\mathrm{~d}, J=8.8 \mathrm{~Hz}, 1 \mathrm{H}), 7.43(\mathrm{~d}, J=2.0 \mathrm{~Hz}, 1 \mathrm{H})$, $7.31\left(\mathrm{dd}, J_{1}=8.8 \mathrm{~Hz}, J_{2}=2.4 \mathrm{~Hz}, 1 \mathrm{H}\right), 4.00(\mathrm{~s}, 3 \mathrm{H}), 1.83(\mathrm{~s}, 9 \mathrm{H})$.

${ }^{13}$ C NMR (100 MHz, CDCl $\left.\mathbf{3}\right) \delta 164.5,155.9,145.9,126.7,122.1,114.4,107.3$, $64.9,56.0,28.6$.

HRMS (ESI-TOF) m/z: $[\mathrm{M}+\mathrm{Na}]^{+}$Calcd for $\mathrm{C}_{12} \mathrm{H}_{15} \mathrm{~N}_{3} \mathrm{ONa}$ 256.1062; Found 256.1029 .

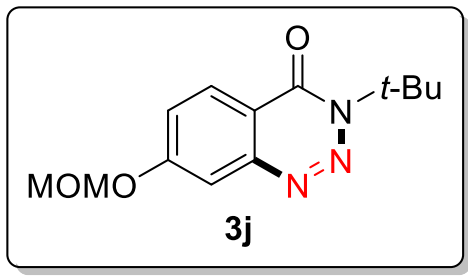

\section{3-(tert-butyl)-7-(methoxymethoxy)benzo[d] $[1,2,3]$ triazin-4(3H)-one}

3j: White gum, (94 mg, 72\% yield on $0.5 \mathrm{mmol} \mathrm{scale})$

TLC: $\mathrm{R}_{\mathrm{f}}=0.4($ Petroleum ether $/$ EtOAc $=10 / 1)$

${ }^{1}$ H NMR (400 MHz, CDCl 3$) \delta 8.27(\mathrm{~d}, J=8.8 \mathrm{~Hz}, 1 \mathrm{H}), 7.66(\mathrm{~d}, J=2.4 \mathrm{~Hz}, 1 \mathrm{H})$, $7.41\left(\mathrm{dd}, J_{1}=8.8 \mathrm{~Hz}, J_{2}=2.8 \mathrm{~Hz}, 1 \mathrm{H}\right), 5.36(\mathrm{~s}, 2 \mathrm{H}), 3.54(\mathrm{~s}, 3 \mathrm{H}), 1.82(\mathrm{~s}, 9 \mathrm{H})$.

${ }^{13}$ C NMR (100 MHz, $\left.\mathbf{C D C l}_{3}\right) \delta 161.9,155.9,145.7,126.8,122.4,115.1,110.9$, 94.4, 64.9, 56.5, 28.6 .

HRMS (ESI-TOF) m/z: $[\mathrm{M}+\mathrm{Na}]^{+}$Calcd for $\mathrm{C}_{13} \mathrm{H}_{17} \mathrm{~N}_{3} \mathrm{O}_{3} \mathrm{Na}$ 286.1168; Found 286.1192 .

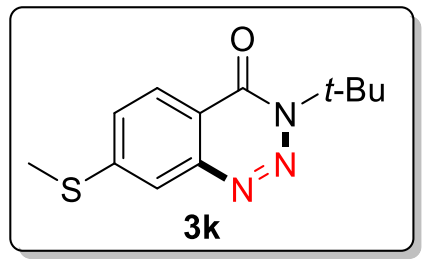

3-(tert-butyl)-7-(methylthio)benzo[d] $[1,2,3]$ triazin-4(3H)-one 
3k: Orange solid, m. p. $115.8-118.2{ }^{\circ} \mathrm{C}(69 \mathrm{mg}, 56 \%$ yield on $0.5 \mathrm{mmol}$ scale $)$

TLC: $\mathrm{R}_{\mathrm{f}}=0.4($ Petroleum ether/EtOAc $=10 / 1)$

${ }^{1}$ H NMR (400 MHz, CDCl $) \delta 8.18(\mathrm{~d}, J=8.8 \mathrm{~Hz}, 1 \mathrm{H}), 7.78(\mathrm{~d}, J=2.0 \mathrm{~Hz}, 1 \mathrm{H})$, $7.57\left(\mathrm{dd}, J_{1}=8.4 \mathrm{~Hz}, J_{2}=2.0 \mathrm{~Hz}, 1 \mathrm{H}\right), 2.64(\mathrm{~s}, 3 \mathrm{H}), 1.83(\mathrm{~s}, 9 \mathrm{H})$.

${ }^{13}$ C NMR (100 MHz, $\left.\mathbf{C D C l}_{3}\right) \delta 155.9,148.4,144.3,129.5,124.9,121.2,117.2$, $65.1,28.6,14.9$.

HRMS (ESI-TOF) m/z: $[\mathrm{M}+\mathrm{Na}]^{+}$Calcd for $\mathrm{C}_{12} \mathrm{H}_{15} \mathrm{~N}_{3} \mathrm{OSNa} 272.0834$; Found 272.0838 .

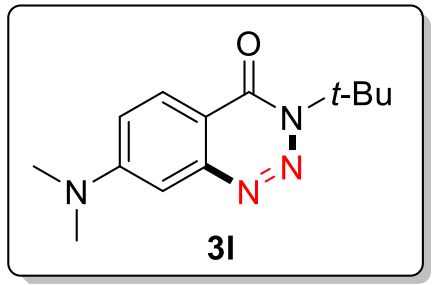

\section{3-(tert-butyl)-7-(dimethylamino)benzo $[d][1,2,3]$ triazin-4(3H)-one}

31: Light yellow solid, m. p. $124.7-126.2{ }^{\circ} \mathrm{C}(83 \mathrm{mg}, 67 \%$ yield on $0.5 \mathrm{mmol}$ scale $)$ TLC: $R_{\mathrm{f}}=0.4($ Petroleum ether $/$ EtOAc $=2 / 1)$

${ }^{1}$ H NMR (600 MHz, CDCl 3$) \delta 8.12(\mathrm{~d}, J=8.4 \mathrm{~Hz}, 1 \mathrm{H}), 7.08-7.05(\mathrm{~m}, 2 \mathrm{H})$, $3.13(\mathrm{~s}, 6 \mathrm{H}), 1.79(\mathrm{~s}, 9 \mathrm{H})$.

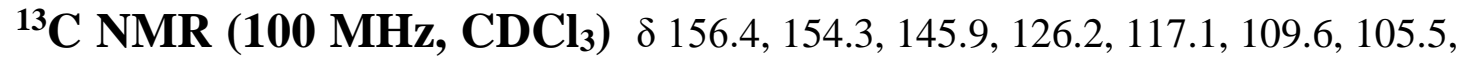
$64.4,40.3,28.8$.

HRMS (ESI-TOF) m/z: $[\mathrm{M}+\mathrm{Na}]^{+}$Calcd for $\mathrm{C}_{13} \mathrm{H}_{18} \mathrm{~N}_{4} \mathrm{ONa}$ 269.1378; Found 269.1365 .

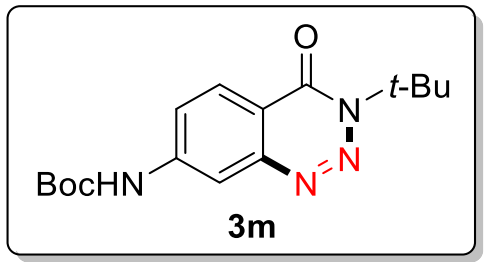

tert-butyl 
(3-(tert-butyl)-4-oxo-3,4-dihydrobenzo $[d][1,2,3]$ triazin-7-yl)carbamat

e

3m: Pale yellow solid, m. p. $185.2-185.8^{\circ} \mathrm{C}(88 \mathrm{mg}, 55 \%$ yield on $0.5 \mathrm{mmol}$ scale $)$

TLC: $\mathrm{R}_{\mathrm{f}}=0.6($ Petroleum ether $/$ EtOAc $=5 / 1)$

${ }^{1} \mathbf{H}$ NMR (400 MHz, CDCl $) \delta 8.22(\mathrm{~d}, J=8.8 \mathrm{~Hz}, 1 \mathrm{H}), 8.12(\mathrm{~d}, J=2.4 \mathrm{~Hz}, 1 \mathrm{H})$, $7.76(\mathrm{dd}, J=8.4,2.0 \mathrm{~Hz}, 1 \mathrm{H}), 7.20$ (s, 1H), 1.79 (s, 9H), 1.53 (s, 9H).

${ }^{13}$ C NMR (100 MHz, CDCl $) \delta 155.9,152.1,145.1,144.4,126.3,122.4,115.5$, $113.9,81.8,64.9,28.6,28.3$.

HRMS (ESI-TOF) m/z: $[\mathrm{M}+\mathrm{H}]^{+}$Calcd for $\mathrm{C}_{16} \mathrm{H}_{23} \mathrm{~N}_{4} \mathrm{O}_{3}$ 319.1770; Found 319.1776 .

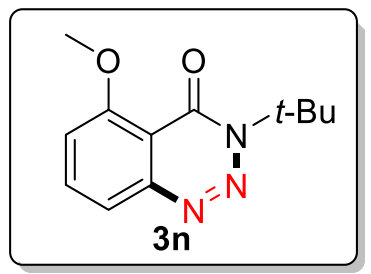

\section{3-(tert-butyl)-5-methoxybenzo[d][1,2,3]triazin-4(3H)-one}

3n: Pale yellow solid, m. p. $109.3-112.8^{\circ} \mathrm{C}(63 \mathrm{mg}, 54 \%$ yield on $0.5 \mathrm{mmol}$ scale $)$

TLC: $\mathrm{R}_{\mathrm{f}}=0.3$ (Petroleum ether $/$ EtOAc $\left.=10 / 1\right)$

${ }^{1} \mathbf{H}$ NMR (400 MHz, CDCl 3 ) $\delta 8.21(\mathrm{~d}, J=9.2 \mathrm{~Hz}, 1 \mathrm{H}), 7.40(\mathrm{~d}, J=2.4 \mathrm{~Hz}, 1 \mathrm{H})$, $7.28\left(\mathrm{dd}, J_{1}=8.8 \mathrm{~Hz}, J_{2}=2.4 \mathrm{~Hz}, 1 \mathrm{H}\right), 3.97(\mathrm{~s}, 3 \mathrm{H}), 1.79(\mathrm{~s}, 9 \mathrm{H})$.

${ }^{13}$ C NMR (100 MHz, CDCl $) \delta 164.5,155.9,145.9,126.7,122.1,114.4,107.3$, $64.9,56.0,28.6$.

HRMS (ESI-TOF) m/z: $[\mathrm{M}+\mathrm{Na}]^{+}$Calcd for $\mathrm{C}_{12} \mathrm{H}_{15} \mathrm{~N}_{3} \mathrm{O}_{2} \mathrm{Na}$ 256.1062; Found 256.1056 . 


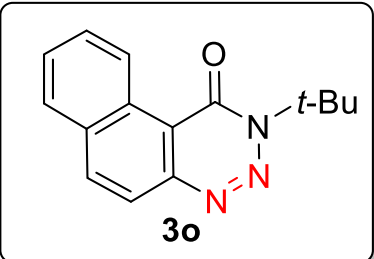

\section{2-(tert-butyl)naphtho[2,1-d] $[1,2,3]$ triazin-1(2H)-one}

3o: Orange solid, m. p. $92.8-94.2{ }^{\circ} \mathrm{C}(90 \mathrm{mg}, 71 \%$ yield on $0.5 \mathrm{mmol}$ scale $)$

TLC: $\mathrm{R}_{\mathrm{f}}=0.2($ Petroleum ether $/$ EtOAc $=20 / 1)$

${ }^{1}$ H NMR (400 MHz, CDCl 3 ) $\delta 9.97$ (d, $J=8.4$ Hz, 1H), $8.33-8.26$ (m, 1H), $8.12-8.06(\mathrm{~m}, 1 \mathrm{H}), 8.02(\mathrm{~d}, J=8.0 \mathrm{~Hz}, 1 \mathrm{H}), 7.89-7.75(\mathrm{~m}, 2 \mathrm{H}), 1.94(\mathrm{~s}, 9 \mathrm{H})$.

${ }^{13}$ C NMR (100 MHz, CDCl 3$) \delta 157.0,144.7,136.2,133.9,129.3,129.0,128.9$, $128.8,128.5,124.2,115.4,65.9,28.7$.

HRMS (ESI-TOF) m/z: $[\mathrm{M}+\mathrm{Na}]^{+}$Calcd for $\mathrm{C}_{15} \mathrm{H}_{15} \mathrm{~N}_{3} \mathrm{ONa}$ 276.1113; Found 276.1125 .

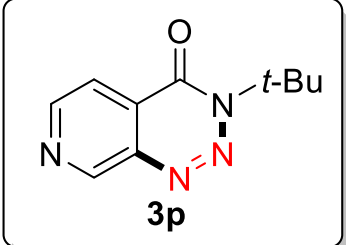

\section{3-(tert-butyl)pyrido[3,4-d][1,2,3]triazin-4(3H)-one}

3p: Orange solid, m. p. $59.8-62.9^{\circ} \mathrm{C}$ ( $46 \mathrm{mg}, 45 \%$ yield on $0.5 \mathrm{mmol}$ scale)

TLC: $R_{\mathrm{f}}=0.5($ Petroleum ether $/$ EtOAc $=5 / 1)$

${ }^{1}$ H NMR (400 MHz, CDCl $) \delta 9.53(\mathrm{~s}, 1 \mathrm{H}), 8.95(\mathrm{~d}, J=5.2 \mathrm{~Hz}, 1 \mathrm{H}), 8.11(\mathrm{~d}, J$ $=5.6 \mathrm{~Hz}, 1 \mathrm{H}), 1.81(\mathrm{~s}, 9 \mathrm{H})$.

${ }^{13}$ C NMR (100 MHz, CDCl 3 ) $\delta$ 154.8, 151.5, 150.9, 138.2, 125.6, 117.1, 65.9, 28.4 .

HRMS (ESI-TOF) m/z: $[\mathrm{M}+\mathrm{H}]^{+}$Calcd for $\mathrm{C}_{10} \mathrm{H}_{13} \mathrm{~N}_{4} \mathrm{O}$ 205.1089; Found 205.1099. 


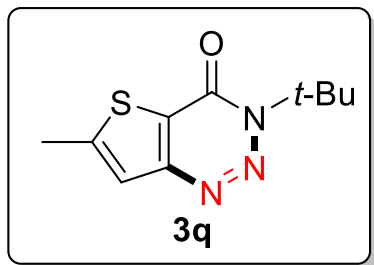

\section{3-(tert-butyl)-6-methylthieno[3,2-d][1,2,3] triazin-4(3H)-one}

3q: White solid, m. p. $70.2-73.3{ }^{\circ} \mathrm{C}(45 \mathrm{mg}, 40 \%$ yield on $0.5 \mathrm{mmol}$ scale $)$

TLC: $\mathrm{R}_{\mathrm{f}}=0.2($ Petroleum ether $/$ EtOAc $=20 / 1)$

${ }^{1}$ H NMR (400 MHz, CDCl 3 ) $\delta 7.20$ (s, 1H), 2.59 (s, 3H), 1.74 (s, 9H).

${ }^{13}$ C NMR (100 MHz, $\left.\mathbf{C D C l}_{3}\right) \delta$ 154.2, 153.8, 150.8, 126.9, 121.9, 65.9, 28.6, 16.6 .

HRMS (ESI-TOF) m/z: $[\mathrm{M}+\mathrm{Na}]^{+}$Calcd for $\mathrm{C}_{10} \mathrm{H}_{13} \mathrm{~N}_{3} \mathrm{OSNa} 246.0677$; Found 246.0675 .

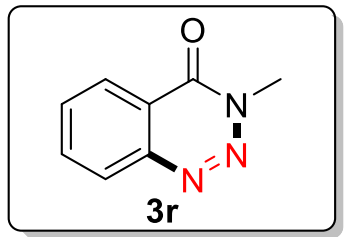

\section{3-methylbenzo $[d][1,2,3]$ triazin-4(3H)-one}

3r: White solid, m. p. $117.8-119.6{ }^{\circ} \mathrm{C}(59 \mathrm{mg}, 73 \%$ yield on $0.5 \mathrm{mmol}$ scale $)$

TLC: $\mathrm{R}_{\mathrm{f}}=0.4($ Petroleum ether $/$ EtOAc $=5 / 1)$

${ }^{1}$ H NMR (600 MHz, CDCl $)_{3} \delta 8.32\left(\mathrm{dd}, J_{1}=8.4 \mathrm{~Hz}, J_{2}=1.0 \mathrm{~Hz}, 1 \mathrm{H}\right), 8.11(\mathrm{~d}$, $J=8.4 \mathrm{~Hz}, 1 \mathrm{H}), 7.94-7.88(\mathrm{~m}, 1 \mathrm{H}), 7.79-7.74(\mathrm{~m}, 1 \mathrm{H}), 4.03(\mathrm{~s}, 3 \mathrm{H})$.

${ }^{13}$ C NMR (100 MHz, $\left.\mathbf{C D C l}_{3}\right) \delta$ 155.9, 144.5, 134.7, 132.3, 128.3, 124.9, 119.7, 37.3.

HRMS (ESI-TOF) m/z: $[\mathrm{M}+\mathrm{H}]^{+}$Calcd for $\mathrm{C}_{8} \mathrm{H}_{8} \mathrm{~N}_{3} \mathrm{O}$ 162.0667; Found 162.0675 . 


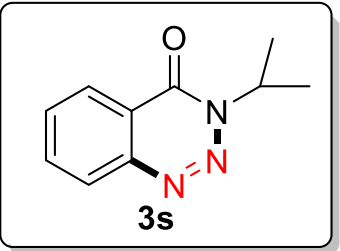

\section{3-isopropylbenzo[d][1,2,3] triazin-4(3H)-one}

3s: White solid, m. p. $52.8-53.2{ }^{\circ} \mathrm{C}(77 \mathrm{mg}, 82 \%$ yield on $0.5 \mathrm{mmol}$ scale $)$

TLC: $\mathrm{R}_{\mathrm{f}}=0.3($ Petroleum ether $/$ EtOAc $=10 / 1)$

${ }^{1} \mathbf{H}$ NMR (400 MHz, CDCl $) \delta 8.33\left(\mathrm{dd}, J_{1}=8.0 \mathrm{~Hz}, J_{2}=1.2 \mathrm{~Hz}, 1 \mathrm{H}\right), 8.12(\mathrm{dd}$, $\left.J_{1}=8.0 \mathrm{~Hz}, J_{2}=0.4 \mathrm{~Hz}, 1 \mathrm{H}\right), 7.94-7.88(\mathrm{~m}, 1 \mathrm{H}), 7.79-7.73(\mathrm{~m}, 1 \mathrm{H}), 5.46-5.39$ $(\mathrm{m}, 1 \mathrm{H}), 1.57(\mathrm{~d}, J=6.8 \mathrm{~Hz}, 6 \mathrm{H})$.

${ }^{13}$ C NMR (100 MHz, CDCl 3$) \delta 155.1,144.0,134.7,132.1,128.1,125.2,119.6$, 49.6, 21.6.

HRMS (ESI-TOF) m/z: $[\mathrm{M}+\mathrm{H}]^{+}$Calcd for $\mathrm{C}_{10} \mathrm{H}_{12} \mathrm{~N}_{3} \mathrm{O}$ 190.0980; Found 190.0986 .

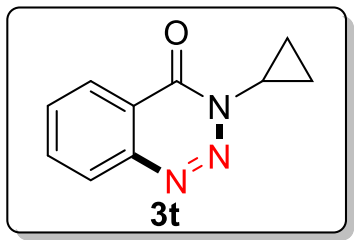

\section{3-cyclopropylbenzo[d] $[1,2,3]$ triazin-4(3H)-one}

3t: Pale yellow solid, m. p. $86.8-90.1^{\circ} \mathrm{C}(67 \mathrm{mg}, 72 \%$ yield on $0.5 \mathrm{mmol}$ scale $)$

TLC: $\mathrm{R}_{\mathrm{f}}=0.2($ Petroleum ether $/$ EtOAc $=20 / 1)$

${ }^{1}$ H NMR (400 MHz, CDCl 3 ) $\delta 8.39(\mathrm{~d}, J=7.2 \mathrm{~Hz}, 1 \mathrm{H}), 8.16(\mathrm{~d}, J=7.6 \mathrm{~Hz}, 1 \mathrm{H})$, $8.04-7.89(\mathrm{~m}, 1 \mathrm{H}), 7.88-7.72(\mathrm{~m}, 1 \mathrm{H}), 4.12-3.89(\mathrm{~m}, 1 \mathrm{H}), 1.38-1.17(\mathrm{~m}, 4 \mathrm{H})$.

${ }^{13}$ C NMR (100 MHz, $\left.\mathbf{C D C l}_{3}\right) \delta$ 156.6, 143.8, 134.8, 132.3, 128.2, 124.9, 119.3, 31.6, 6.6.

HRMS (ESI-TOF) m/z: $[\mathrm{M}+\mathrm{H}]^{+}$Calcd for $\mathrm{C}_{10} \mathrm{H}_{10} \mathrm{~N}_{3} \mathrm{O}$ 188.0824; Found 188.0832 


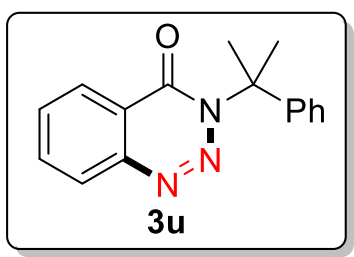

\section{3-(2-phenylpropan-2-yl)benzo[d][1,2,3]triazin-4(3H)-one}

3u: Pale yellow solid, m. p. $87.5-88.7^{\circ} \mathrm{C}(92 \mathrm{mg}, 69 \%$ yield on $0.5 \mathrm{mmol}$ scale; $68 \%$ in $3 \mathrm{mmol}$ scale)

TLC: $\mathrm{R}_{\mathrm{f}}=0.3($ Petroleum ether $/ \mathrm{EtOAc}=20 / 1)$

${ }^{1} \mathbf{H}$ NMR (400 MHz, CDCl $) \delta 8.24-8.09$ (m, 2H), $7.97-7.88$ (m, 1H), $7.76-$ $7.66(\mathrm{~m}, 1 \mathrm{H}), 7.35-7.21(\mathrm{~m}, 5 \mathrm{H}), 2.13(\mathrm{~s}, 6 \mathrm{H})$.

${ }^{13}$ C NMR (100 MHz, $\left.\mathbf{C D C l}_{3}\right) \delta 155.1,145.4,144.0,134.7,132.0,128.5,127.8$, $126.9,125.3,124.3,120.8,67.9,29.1$.

HRMS (ESI-TOF) m/z: $[\mathrm{M}+\mathrm{Na}]^{+}$Calcd for $\mathrm{C}_{16} \mathrm{H}_{15} \mathrm{~N}_{3} \mathrm{ONa}$ 288.1113; Found 288.1121 .

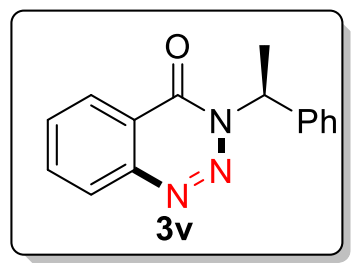

\section{(S)-3-(1-phenylethyl)benzo[d] $[1,2,3]$ triazin-4(3H)-one}

3v: Pale yellow solid, m. p. $57.7-59.9^{\circ} \mathrm{C}(78 \mathrm{mg}, 62 \%$ yield on $0.5 \mathrm{mmol}$ scale $)$

TLC: $\mathrm{R}_{\mathrm{f}}=0.3($ Petroleum ether $/$ EtOAc $=10 / 1)$

${ }^{1} \mathbf{H}$ NMR (600 MHz, CDCl $) \delta 8.32\left(\mathrm{dd}, J_{1}=8.4 \mathrm{~Hz}, J_{2}=1.1 \mathrm{~Hz}, 1 \mathrm{H}\right), 8.13(\mathrm{~d}, \mathrm{~J}$ $=8.4 \mathrm{~Hz}, 1 \mathrm{H}), 7.93-7.89(\mathrm{~m}, 1 \mathrm{H}), 7.79-7.74(\mathrm{~m}, 1 \mathrm{H}), 7.56-7.51(\mathrm{~m}, 2 \mathrm{H}), 7.35-$ $7.32(\mathrm{~m}, 2 \mathrm{H}), 7.29-7.25(\mathrm{~m}, 1 \mathrm{H}), 6.44(\mathrm{q}, J=7.2 \mathrm{~Hz}, 1 \mathrm{H}), 2.02(\mathrm{~d}, J=7.2 \mathrm{~Hz}, 3 \mathrm{H})$.

${ }^{13}$ C NMR (150 MHz, $\left.\mathbf{C D C l}_{3}\right) \delta$ 155.2, 143.9, 140.5, 134.8, 132.2, 128.6, 128.1, $128.0,127.5,125.3,119.7,56.2,20.2$.

HRMS (ESI-TOF) m/z: $[\mathrm{M}+\mathrm{Na}]^{+}$Calcd for $\mathrm{C}_{15} \mathrm{H}_{13} \mathrm{~N}_{3} \mathrm{ONa}$ 274.0956; Found 
<smiles>COc1ccc(-n2nnc3ccccc3c2=O)cc1</smiles>

\section{3-(4-methoxyphenyl)benzo $[d][1,2,3]$ triazin-4(3H)-one}

3w: Pale yellow solid, m. p. $153.5-154.8{ }^{\circ} \mathrm{C}(80 \mathrm{mg}, 63 \%$ yield on $0.5 \mathrm{mmol}$ scale $)$

TLC: $\mathrm{R}_{\mathrm{f}}=0.2($ Petroleum ether/DCM $=2 / 1)$

${ }^{1} \mathbf{H}$ NMR (400 MHz, CDCl 3 ) $\delta 8.43\left(\mathrm{dd}, J_{1}=8.0 \mathrm{~Hz}, J_{2}=1.2 \mathrm{~Hz}, 1 \mathrm{H}\right), 8.21(\mathrm{~d}, J$ $=8.0 \mathrm{~Hz}, 1 \mathrm{H}), 8.02-7.94(\mathrm{~m}, 1 \mathrm{H}), 7.86-7.80(\mathrm{~m}, 1 \mathrm{H}), 7.58-7.52(\mathrm{~m}, 2 \mathrm{H}), 7.09-$ $7.01(\mathrm{~m}, 2 \mathrm{H}), 3.88(\mathrm{~s}, 3 \mathrm{H})$.

${ }^{13}$ C NMR (100 MHz, $\left.\mathbf{C D C l}_{3}\right) \delta$ 159.9, 155.4, 143.8, 135.0, 132.7, 131.7, 128.5, 127.3, 125.6, 120.4, 114.3, 55.6.

HRMS (ESI-TOF) m/z: $[\mathrm{M}+\mathrm{Na}]^{+}$Calcd for $\mathrm{C}_{14} \mathrm{H}_{11} \mathrm{~N}_{3} \mathrm{O}_{2} \mathrm{Na}$ 276.0749; Found 276.0741 .

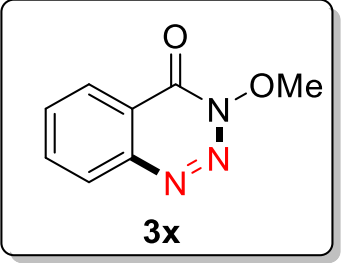

\section{3-methoxybenzo $[d][1,2,3]$ triazin-4(3H)-one}

3x: White solid, m. p. $217.3-220.0{ }^{\circ} \mathrm{C}(45 \mathrm{mg}, 51 \%$ yield on $0.5 \mathrm{mmol}$ scale $)$

TLC: $\mathrm{R}_{\mathrm{f}}=0.2($ Petroleum ether/EtOAc $=5 / 1)$

${ }^{1} \mathbf{H}$ NMR (400 MHz, CDCl $) \delta 8.38\left(\mathrm{dd}, J_{1}=8.0 \mathrm{~Hz}, J_{2}=2.4 \mathrm{~Hz}, 1 \mathrm{H}\right), 8.20(\mathrm{dd}$, $\left.J_{1}=8.0 \mathrm{~Hz}, J_{2}=2.4 \mathrm{~Hz}, 1 \mathrm{H}\right), 8.06-7.93(\mathrm{~m}, 1 \mathrm{H}), 7.88-7.72(\mathrm{~m}, 1 \mathrm{H}), 4.27(\mathrm{~s}, 3 \mathrm{H})$.

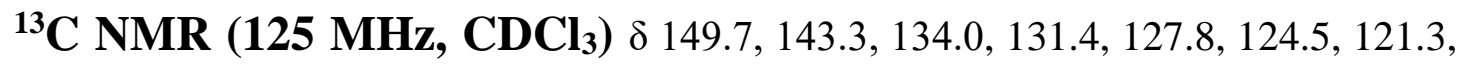
64.7. 
HRMS (ESI-TOF) m/z: $[\mathrm{M}+\mathrm{Na}]^{+}$Calcd for $\mathrm{C}_{8} \mathrm{H}_{7} \mathrm{~N}_{3} \mathrm{O}_{2} \mathrm{Na}$ 200.0436; Found 200.0433.

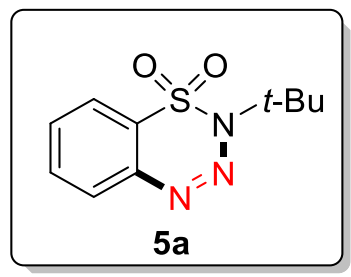

\section{2-(tert-butyl)-2H-benzo[e][1,2,3,4]thiatriazine 1,1-dioxide}

5a: Pale orange solid, m. p. $78.4-82.3^{\circ} \mathrm{C}(81 \mathrm{mg}, 68 \%$ yield on $0.5 \mathrm{mmol}$ scale $)$

TLC: $\mathrm{R}_{\mathrm{f}}=0.3($ Petroleum ether $/ \mathrm{EtOAc}=20 / 1)$

${ }^{1}$ H NMR (400 MHz, CDCl $) \delta 8.02-7.94(\mathrm{~m}, 2 \mathrm{H}), 7.87-7.80(\mathrm{~m}, 1 \mathrm{H}), 7.76-$ $7.70(\mathrm{~m}, 1 \mathrm{H}), 1.81(\mathrm{~s}, 9 \mathrm{H})$.

${ }^{13}$ C NMR (100 MHz, $\left.\mathbf{C D C l}_{3}\right) \delta$ 141.1, 133.9, 132.2, 128.5, 126.2, 120.3, 67.8, 30.4 .

HRMS (ESI-TOF) m/z: $[\mathrm{M}+\mathrm{Na}]^{+}$Calcd for $\mathrm{C}_{10} \mathrm{H}_{13} \mathrm{~N}_{3} \mathrm{O}_{2} \mathrm{SNa}$ 262.0626; Found 262.0625 .

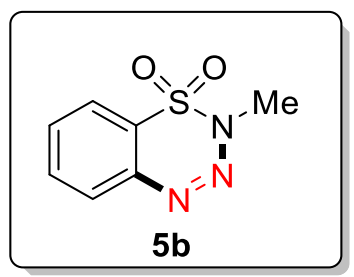

\section{2-methyl-2H-benzo[e][1,2,3,4]thiatriazine 1,1-dioxide}

5b: Orange solid, m. p. $68.3-71.8^{\circ} \mathrm{C}(45 \mathrm{mg}, 46 \%$ yield on $0.5 \mathrm{mmol}$ scale $)$

TLC: $\mathrm{R}_{\mathrm{f}}=0.1($ Petroleum ether $/ \mathrm{EtOAc}=10 / 1)$

${ }^{1}$ H NMR (400 MHz, CDCl $) \delta 8.09-8.04$ (m, 2H), 7.95 - 7.90 (m, 1H), 7.85 $7.79(\mathrm{~m}, 1 \mathrm{H}), 3.94(\mathrm{~s}, 3 \mathrm{H})$.

${ }^{13}$ C NMR (100 MHz, CDCl 3 ) $\delta$ 141.7, 134.2, 132.7, 129.4, 125.3, 120.5, 33.9. 
HRMS (ESI-TOF) m/z: $[\mathrm{M}+\mathrm{Na}]^{+}$Calcd for $\mathrm{C}_{7} \mathrm{H}_{7} \mathrm{~N}_{3} \mathrm{O}_{2} \mathrm{SNa}$ 220.0157; Found 220.0156 .

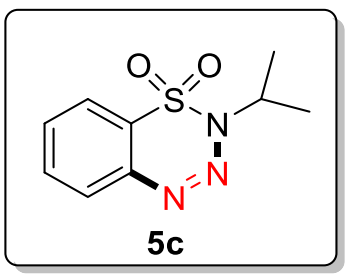

\section{2-isopropyl-2H-benzo[e][1,2,3,4] thiatriazine 1,1-dioxide}

5c: Yellow solid, m. p. $92.5-95.0{ }^{\circ} \mathrm{C}(57 \mathrm{mg}, 51 \%$ yield on $0.5 \mathrm{mmol}$ scale $)$

TLC: $\mathrm{R}_{\mathrm{f}}=0.2($ Petroleum ether $/$ EtOAc $=10 / 1)$

${ }^{1}$ H NMR (400 MHz, CDCl $) \delta 8.09-8.02(\mathrm{~m}, 2 \mathrm{H}), 7.94-7.87(\mathrm{~m}, 1 \mathrm{H}), 7.82-$ $7.76(\mathrm{~m}, 1 \mathrm{H}), 4.98$ (hept, $J=6.8 \mathrm{~Hz}, 1 \mathrm{H}), 1.70$ (s, 3H), 1.68 (s, 3H).

${ }^{13}$ C NMR (100 MHz, $\left.\mathbf{C D C l}_{3}\right) \delta$ 141.5, 134.0, 132.3, 129.1, 125.3, 120.4, 50.7, 23.1.

HRMS (ESI-TOF) m/z: $[\mathrm{M}+\mathrm{Na}]^{+}$Calcd for $\mathrm{C}_{9} \mathrm{H}_{11} \mathrm{~N}_{3} \mathrm{O}_{2} \mathrm{SNa}$ 248.0470; Found 248.0474 .

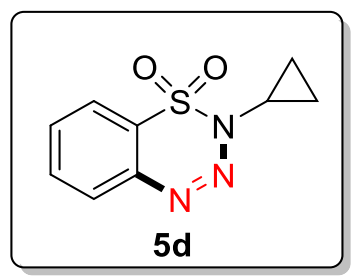

\section{2-cyclopropyl-2H-benzo[e][1,2,3,4]thiatriazine 1,1-dioxide}

5d: Orange solid, m. p. $80.1-83.9^{\circ} \mathrm{C}(51 \mathrm{mg}, 45 \%$ yield on $0.5 \mathrm{mmol}$ scale $)$

TLC: $\mathrm{R}_{\mathrm{f}}=0.2($ Petroleum ether $/$ EtOAc $=10 / 1)$

${ }^{1}$ H NMR (400 MHz, CDCl $)$ ) 8.07 - 7.98 (m, 2H), 7.91 - 7.85 (m, 1H), 7.81 $7.74(\mathrm{~m}, 1 \mathrm{H}), 3.63-3.50(\mathrm{~m}, 1 \mathrm{H}), 1.27-1.16(\mathrm{~m}, 4 \mathrm{H})$.

${ }^{13}$ C NMR (100 MHz, $\left.\mathbf{C D C l} 3\right) \delta$ 141.1, 134.2, 132.7, 129.4, 125.9, 120.6, 28.6, 6.6. 
HRMS (ESI-TOF) m/z: $[\mathrm{M}+\mathrm{Na}]^{+}$Calcd for $\mathrm{C}_{9} \mathrm{H}_{9} \mathrm{~N}_{3} \mathrm{O}_{2} \mathrm{SNa}$ 246.0313; Found 246.0316 .

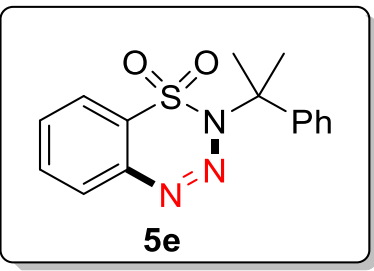

\section{2-(2-phenylpropan-2-yl)-2H-benzo[e][1,2,3,4]thiatriazine 1,1-dioxide}

5e: Pale yellow solid, m. p. $90.0-92.2^{\circ} \mathrm{C}(63 \mathrm{mg}, 42 \%$ yield on $0.5 \mathrm{mmol}$ scale $)$

TLC: $\mathrm{R}_{\mathrm{f}}=0.3($ Petroleum ether $/ \mathrm{EtOAc}=20 / 1)$

${ }^{1}$ H NMR (400 MHz, CDCl $) \delta 8.00-7.92(\mathrm{~m}, 2 \mathrm{H}), 7.89-7.80(\mathrm{~m}, 1 \mathrm{H}), 7.78-$ $7.68(\mathrm{~m}, 1 \mathrm{H}), 7.42(\mathrm{~d}, J=7.6 \mathrm{~Hz}, 2 \mathrm{H}), 7.37-7.21(\mathrm{~m}, 3 \mathrm{H}), 2.20$ (s, 6H).

${ }^{13}$ C NMR (100 MHz, CDCl 3 ) $\delta$ 145.4, 141.0, 133.9, 132.4, 128.7, 128.4, 127.5, $126.5,125.5,120.3,70.9,30.3$.

HRMS (ESI-TOF) m/z: $[\mathrm{M}+\mathrm{Na}]^{+}$Calcd for $\mathrm{C}_{15} \mathrm{H}_{15} \mathrm{~N}_{3} \mathrm{O}_{2} \mathrm{SNa}$ 324.0783; Found 324.0786 .

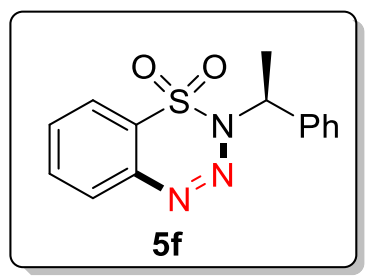

\section{(S)-2-(1-phenylethyl)-2H-benzo[e][1,2,3,4]thiatriazine 1,1-dioxide}

5f: Pale orange solid, m. p. $83.2-85.0^{\circ} \mathrm{C}(80 \mathrm{mg}, 56 \%$ yield on $0.5 \mathrm{mmol}$ scale $)$

TLC: $\mathrm{R}_{\mathrm{f}}=0.3($ Petroleum ether/EtOAc $=8 / 1)$

${ }^{1}$ H NMR (400 MHz, CDCl 3 ) $\delta 8.07-7.94(\mathrm{~m}, 2 \mathrm{H}), 7.89-7.81(\mathrm{~m}, 1 \mathrm{H}), 7.79-$

$7.71(\mathrm{~m}, 1 \mathrm{H}), 7.55(\mathrm{~d}, J=7.2 \mathrm{~Hz}, 2 \mathrm{H}), 7.40-7.28(\mathrm{~m}, 3 \mathrm{H}), 5.92(\mathrm{q}, J=7.2 \mathrm{~Hz}, 1 \mathrm{H})$, $2.09(\mathrm{~d}, J=7.2 \mathrm{~Hz}, 3 \mathrm{H})$. 
${ }^{13}$ C NMR (100 MHz, CDCl $) \delta$ 141.5, 139.8, 134.1, 132.4, 129.2, 128.7, 128.4, 127.6, 125.4, 120.5, 56.6, 21.4 .

HRMS (ESI-TOF) m/z: $[\mathrm{M}+\mathrm{Na}]^{+}$Calcd for $\mathrm{C}_{14} \mathrm{H}_{13} \mathrm{~N}_{3} \mathrm{O}_{2} \mathrm{SNa}$ 310.0626; Found 310.0629 .

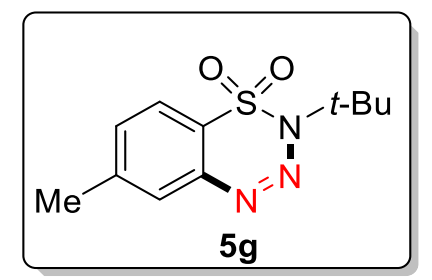

2-(tert-butyl)-6-methyl-2H-benzo[e][1,2,3,4] thiatriazine 1,1-dioxide

5g: Pale orange solid, m. p. $75.5-78.5^{\circ} \mathrm{C}(65 \mathrm{mg}, 51 \%$ yield on $0.5 \mathrm{mmol}$ scale $)$

TLC: $\mathrm{R}_{\mathrm{f}}=0.5($ Petroleum ether $/$ EtOAc $=20 / 1)$

${ }^{1} \mathbf{H}$ NMR (400 MHz, CDCl $) \delta 7.93(\mathrm{~d}, J=8.0 \mathrm{~Hz}, 1 \mathrm{H}), 7.81$ (s, 1H), 7.59 (d, $J$ $=8.0 \mathrm{~Hz}, 1 \mathrm{H}), 2.60(\mathrm{~s}, 3 \mathrm{H}), 1.86(\mathrm{~s}, 9 \mathrm{H})$.

${ }^{13}$ C NMR (100 MHz, $\left.\mathbf{C D C l}_{3}\right) \delta$ 145.2, 141.1, 133.2, 128.4, 123.5, 120.2, 67.6, 30.4, 21.7.

HRMS (ESI-TOF) $\mathbf{m} / \mathbf{z}:[\mathrm{M}+\mathrm{Na}]^{+}$Calcd for $\mathrm{C}_{11} \mathrm{H}_{15} \mathrm{~N}_{3} \mathrm{O}_{2} \mathrm{SNa}$ 276.0783; Found 276.0781 .

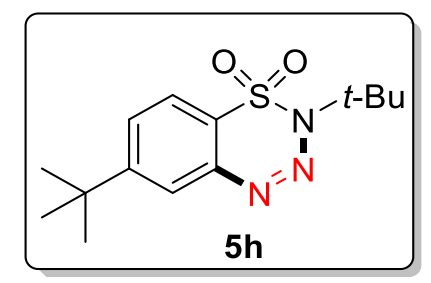

\section{2,6-di-tert-butyl-2H-benzo[e][1,2,3,4]thiatriazine 1,1-dioxide}

5h: Pale yellow solid, m. p. $85.4-87.6{ }^{\circ} \mathrm{C}(72 \mathrm{mg}, 49 \%$ yield on $0.5 \mathrm{mmol}$ scale $)$

TLC: $\mathrm{R}_{\mathrm{f}}=0.5($ Petroleum ether/EtOAc $=20 / 1)$

${ }^{1}$ H NMR (400 MHz, CDCl $) \delta 8.02-7.87(\mathrm{~m}, 2 \mathrm{H}), 7.76(\mathrm{~d}, J=8.0 \mathrm{~Hz}, 1 \mathrm{H})$, $1.81(\mathrm{~s}, 9 \mathrm{H}), 1.40(\mathrm{~s}, 9 \mathrm{H})$. 
${ }^{13}$ C NMR (100 MHz, $\left.\mathbf{C D C l}_{3}\right) \delta$ 158.3, 141.2, 129.8, 125.1, 123.4, 120.1, 67.5, $35.5,31.00,30.4$.

HRMS (ESI-TOF) m/z: $[\mathrm{M}+\mathrm{Na}]^{+}$Calcd for $\mathrm{C}_{14} \mathrm{H}_{21} \mathrm{~N}_{3} \mathrm{O}_{2} \mathrm{SNa}$ 318.1252; Found 318.1252 .

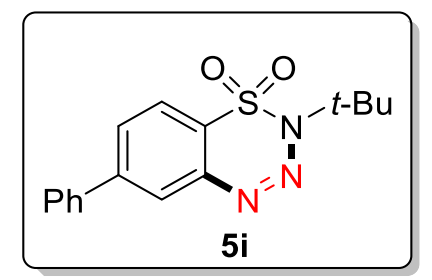

2-(tert-butyl)-6-phenyl-2H-benzo[e][1,2,3,4] thiatriazine 1,1-dioxide

5i: Pale orange solid, m. p. $91.9-95.4^{\circ} \mathrm{C}(83 \mathrm{mg}, 53 \%$ yield on $0.5 \mathrm{mmol}$ scale $)$

TLC: $\mathrm{R}_{\mathrm{f}}=0.3($ Petroleum ether $/ \mathrm{EtOAc}=20 / 1)$

${ }^{1}$ H NMR (400 MHz, CDCl $) \delta 8.16(\mathrm{~s}, 1 \mathrm{H}), 8.06(\mathrm{~d}, J=8.0 \mathrm{~Hz}, 1 \mathrm{H}), 7.94(\mathrm{~d}, J$ $=7.6 \mathrm{~Hz}, 1 \mathrm{H}), 7.66(\mathrm{~d}, J=7.2 \mathrm{~Hz}, 2 \mathrm{H}), 7.57-7.45(\mathrm{~m}, 3 \mathrm{H}), 1.84(\mathrm{~s}, 9 \mathrm{H})$.

${ }^{13}$ C NMR (100 MHz, $\left.\mathbf{C D C l}_{3}\right) \delta$ 147.3, 141.6, 138.2, 130.8, 129.4, 129.2, 127.4, $126.6,124.5,120.9,67.8,30.5$.

HRMS (ESI-TOF) m/z: $[\mathrm{M}+\mathrm{Na}]^{+}$Calcd for $\mathrm{C}_{16} \mathrm{H}_{17} \mathrm{~N}_{3} \mathrm{O}_{2} \mathrm{SNa}$ 338.0939; Found 338.0939 .

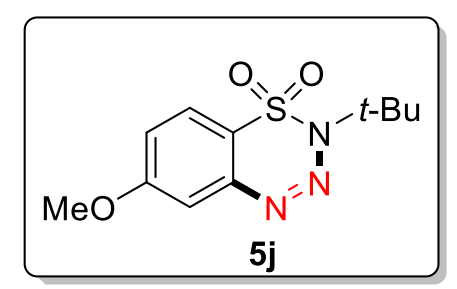

2-(tert-butyl)-6-methoxy-2H-benzo[e][1,2,3,4] thiatriazine 1,1-dioxide

5i: Pale yellow solid, m. p. $121.9-125.5^{\circ} \mathrm{C}(66 \mathrm{mg}, 49 \%$ yield on $0.5 \mathrm{mmol}$ scale $)$

TLC: $\mathrm{R}_{\mathrm{f}}=0.2($ Petroleum ether $/$ EtOAc $=10 / 1)$

${ }^{1} \mathbf{H}$ NMR $\left(\mathbf{6 0 0} \mathbf{M H z}, \mathbf{C D C l}_{3}\right) \delta 7.88(\mathrm{~d}, J=9.0 \mathrm{~Hz}, 1 \mathrm{H}), 7.36(\mathrm{~d}, J=1.8 \mathrm{~Hz}, 1 \mathrm{H})$, $7.23\left(\mathrm{dd}, J_{1}=9.0 \mathrm{~Hz}, J_{2}=1.8 \mathrm{~Hz}, 1 \mathrm{H}\right), 3.94(\mathrm{~s}, 3 \mathrm{H}), 1.80(\mathrm{~s}, 9 \mathrm{H})$. 
${ }^{13}$ C NMR (150 MHz, $\left.\mathbf{C D C l}_{3}\right) \delta 163.7,143.1,122.1,120.2,118.6,110.6,67.6$, $56.1,30.4$.

HRMS (ESI-TOF) $\mathbf{m} / \mathbf{z}:[\mathrm{M}+\mathrm{Na}]^{+}$Calcd for $\mathrm{C}_{11} \mathrm{H}_{15} \mathrm{~N}_{3} \mathrm{O}_{3} \mathrm{SNa}$ 292.0732; Found 292.0733.

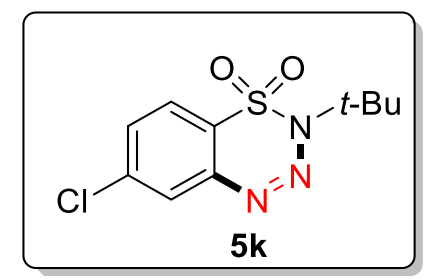

\section{2-(tert-butyl)-6-chloro-2H-benzo[e][1,2,3,4] thiatriazine 1,1-dioxide}

5k: Orange solid, m. p. $81.1-84.0{ }^{\circ} \mathrm{C}(49 \mathrm{mg}, 36 \%$ yield on $0.5 \mathrm{mmol}$ scale $)$

TLC: $\mathrm{R}_{\mathrm{f}}=0.5($ Petroleum ether $/ \mathrm{EtOAc}=20 / 1)$

${ }^{1} \mathbf{H}$ NMR (400 MHz, CDCl 3$) \delta 8.00(\mathrm{~d}, J=2.0 \mathrm{~Hz}, 1 \mathrm{H}), 7.97(\mathrm{~d}, J=8.8 \mathrm{~Hz}, 1 \mathrm{H})$, $7.73\left(\mathrm{dd}, J_{1}=8.8 \mathrm{~Hz}, J_{1}=2.0 \mathrm{~Hz}, 1 \mathrm{H}\right), 1.84(\mathrm{~s}, 9 \mathrm{H})$.

${ }^{13}$ C NMR (100 MHz, $\left.\mathbf{C D C l}_{3}\right) \delta$ 141.9, 140.3, 132.4, 128.1, 124.3, 122.0, 68.4, 30.4 .

HRMS (ESI-TOF) m/z: $\left[\mathrm{M}+\mathrm{Na}^{+}\right.$Calcd for $\mathrm{C}_{10} \mathrm{H}_{12} \mathrm{ClN}_{3} \mathrm{O}_{2} \mathrm{SNa}$ 296.0236; Found 296.0240.

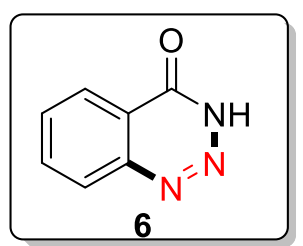

\section{benzo $[d][1,2,3]$ triazin-4(3H)-one}

6: Brown solid, m. p. $210.5-213.0{ }^{\circ} \mathrm{C}(241 \mathrm{mg}, 82 \%$ yield on $2.0 \mathrm{mmol}$ scale $)$

TLC: $\mathrm{R}_{\mathrm{f}}=0.2($ Petroleum ether $/$ EtOAc $=4 / 1)$

${ }^{1}$ H NMR (400 MHz, DMSO-d $\left.\boldsymbol{d}_{6}\right) \delta 15.00(\mathrm{~s}, 1 \mathrm{H}), 8.29-8.16(\mathrm{~m}, 2 \mathrm{H}), 8.10(\mathrm{t}, J$ $=7.2 \mathrm{~Hz}, 1 \mathrm{H}), 7.93(\mathrm{t}, J=7.2 \mathrm{~Hz}, 1 \mathrm{H})$. 
${ }^{13}$ C NMR (150 MHz, DMSO-d 6 ) $\delta$ 156.1, 144.7, 135.9, 133.0, 128.3, 124.7, 120.7 .

HRMS (ESI-TOF) m/z: $[\mathrm{M}+\mathrm{Na}]^{+}$Calcd for $\mathrm{C}_{7} \mathrm{H}_{5} \mathrm{~N}_{3} \mathrm{ONa}$ 170.0330; Found 170.0330 .

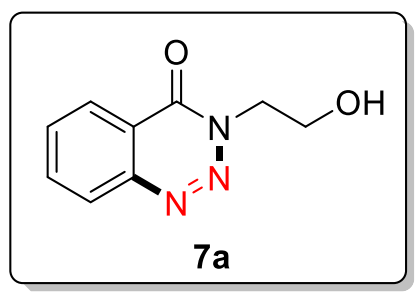

\section{3-(2-hydroxyethyl)benzo $[d][1,2,3]$ triazin- $4(3 H)$-one}

7a: White solid, m. p. $111.3-113.0^{\circ} \mathrm{C}(36 \mathrm{mg}, 95 \%$ yield on $0.2 \mathrm{mmol}$ scale $)$

TLC: $\mathrm{R}_{\mathrm{f}}=0.1($ Petroleum ether $/$ EtOAc $=5 / 1)$

${ }^{1} \mathbf{H}$ NMR (400 MHz, CDCl 3$) \delta 8.30\left(\mathrm{dd}, J_{1}=8.0 \mathrm{~Hz}, J_{2}=0.8 \mathrm{~Hz}, 1 \mathrm{H}\right), 8.12(\mathrm{~d}, J$ $=8.4 \mathrm{~Hz}, 1 \mathrm{H}), 7.97-7.89(\mathrm{~m}, 1 \mathrm{H}), 7.80-7.76(\mathrm{~m}, 1 \mathrm{H}), 4.65(\mathrm{t}, J=5.2 \mathrm{~Hz}, 2 \mathrm{H}), 4.12$ (t, $J=5.2 \mathrm{~Hz}, 2 \mathrm{H}), 2.89(\mathrm{~s}, 1 \mathrm{H})$.

${ }^{13}$ C NMR (100 MHz, $\left.\mathbf{C D C l}_{3}\right) \delta$ 156.5, 144.2, 135.1, 132.6, 128.3, 125.1, 119.6, 61.3, 52.6.

HRMS (ESI-TOF) m/z: $\left[\mathrm{M}+\mathrm{Na}^{+}\right.$Calcd for $\mathrm{C}_{9} \mathrm{H}_{9} \mathrm{~N}_{3} \mathrm{O}_{2} \mathrm{Na}$ 214.0592; Found 214.0590.

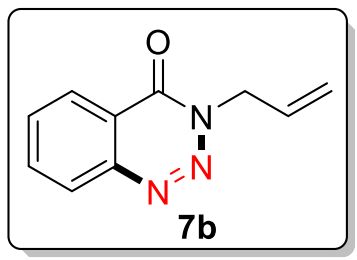

\section{3-allylbenzo $[d][1,2,3]$ triazin-4(3H)-one}

7b: Pale yellow solid, m. p. $66.3-68.9^{\circ} \mathrm{C}(36 \mathrm{mg}, 97 \%$ yield on $0.2 \mathrm{mmol}$ scale $)$

TLC: $\mathrm{R}_{\mathrm{f}}=0.7$ (Petroleum ether/EtOAc $\left.=4 / 1\right)$ 
${ }^{1} \mathbf{H}$ NMR (400 MHz, CDCl $) \delta 8.36(\mathrm{~d}, J=7.6 \mathrm{~Hz}, 1 \mathrm{H}), 8.16(\mathrm{~d}, J=8.0 \mathrm{~Hz}, 1 \mathrm{H})$, $7.99-7.89(\mathrm{~m}, 1 \mathrm{H}), 7.84-7.74(\mathrm{~m}, 1 \mathrm{H}), 6.15-6.00(\mathrm{~m}, 1 \mathrm{H}), 5.42-5.22(\mathrm{~m}, 2 \mathrm{H})$, $5.08(\mathrm{~d}, J=5.6 \mathrm{~Hz}, 2 \mathrm{H})$.

${ }^{13}$ C NMR (100 MHz, $\left.\mathbf{C D C l}_{3}\right) \delta$ 155.2, 144.3, 134.8, 132.3, 131.4, 128.3, 125.0, 119.8, 119.2, 52.1 .

HRMS (ESI-TOF) m/z: $[\mathrm{M}+\mathrm{Na}]^{+}$Calcd for $\mathrm{C}_{10} \mathrm{H}_{9} \mathrm{~N}_{3} \mathrm{ONa}$ 210.0643; Found 210.0642 .

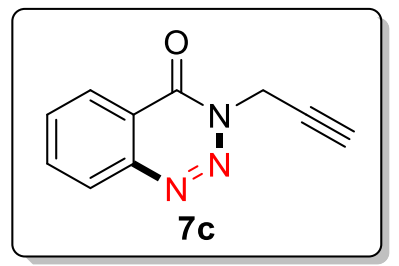

\section{3-(prop-2-yn-1-yl)benzo[d][1,2,3]triazin-4(3H)-one}

7c: White solid, m. p. $117.0-119.5^{\circ} \mathrm{C}$ ( $35 \mathrm{mg}, 94 \%$ yield on $0.2 \mathrm{mmol}$ scale)

TLC: $\mathrm{R}_{\mathrm{f}}=0.6($ Petroleum ether/EtOAc $=4 / 1)$

${ }^{1}$ H NMR (400 MHz, CDCl $) \delta 8.39(\mathrm{~d}, J=8.0 \mathrm{~Hz}, 1 \mathrm{H}), 8.19(\mathrm{~d}, J=8.0 \mathrm{~Hz}, 1 \mathrm{H})$, $8.00-7.60(\mathrm{~m}, 1 \mathrm{H}), 7.86-7.81(\mathrm{~m}, 1 \mathrm{H}), 5.24(\mathrm{~d}, J=2.0 \mathrm{~Hz}, 2 \mathrm{H}), 2.40(\mathrm{~s}, 1 \mathrm{H})$.

${ }^{13}$ C NMR (100 MHz, $\left.\mathbf{C D C l}_{3}\right) \delta 154.9,144.3,135.15,132.7,128.6,125.2,119.9$, $76.9,73.3,39.4$

HRMS (ESI-TOF) m/z: $[\mathrm{M}+\mathrm{Na}]^{+}$Calcd for $\mathrm{C}_{10} \mathrm{H}_{7} \mathrm{~N}_{3} \mathrm{ONa}$ 208.0487; Found 208.0484 .

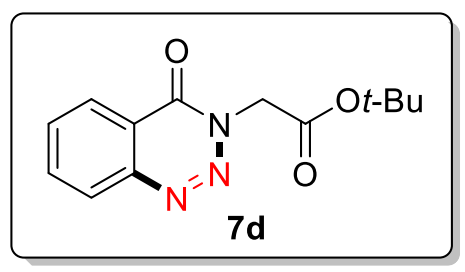

tert-butyl 2-(4-oxobenzo $[d][1,2,3]$ triazin-3(4H)-yl)acetate

7d: White solid, m. p. $86.5-87.9^{\circ} \mathrm{C}(44 \mathrm{mg}, 85 \%$ yield on $0.2 \mathrm{mmol}$ scale $)$ 
TLC: $\mathrm{R}_{\mathrm{f}}=0.4($ Petroleum ether $/$ EtOAc $=10 / 1)$

${ }^{1} \mathbf{H}$ NMR (400 MHz, CDCl $) \delta 8.35\left(\mathrm{dd}, J_{1}=8,0, J_{2}=1.2 \mathrm{~Hz}, 1 \mathrm{H}\right), 8.16(\mathrm{~d}, J=$ $8.0 \mathrm{~Hz}, 1 \mathrm{H}), 7.99-7.90(\mathrm{~m}, 1 \mathrm{H}), 7.83-7.75(\mathrm{~m}, 1 \mathrm{H}), 5.09$ (s, 2H), 1.48 (s, 9H).

${ }^{13}$ C NMR (100 MHz, CDCl $) \delta$ 166.1, 155.6, 144.4, 135.1, 132.6, 128.5, 125.2, $119.8,83.1,51.5,28.0$.

HRMS (ESI-TOF) m/z: $[\mathrm{M}+\mathrm{Na}]^{+}$Calcd for $\mathrm{C}_{13} \mathrm{H}_{15} \mathrm{~N}_{3} \mathrm{O}_{3} \mathrm{Na}$ 284.1011; Found 284.1010.<smiles>[NH2+]CCn1nnc2ccccc2c1=O</smiles>

\section{2-(2-(4-oxobenzo $[d][1,2,3]$ triazin-3(4H)-yl)ethyl)isoindoline-1,3-dione}

7e: White solid, m. p. $218.7-219.5$ (40mg, $62 \%$ yield on $0.2 \mathrm{mmol}$ scale)

TLC: $\mathrm{R}_{\mathrm{f}}=0.3($ Petroleum ether/EtOAc $=4 / 1)$

${ }^{1} \mathbf{H}$ NMR $(500$ MHz, CDCl 3$) \delta 8.34\left(\mathrm{dd}, J_{1}=8.0 \mathrm{~Hz}, J_{2}=1.0 \mathrm{~Hz}, 1 \mathrm{H}\right), 8.09(\mathrm{~d}, J$ $=7.5 \mathrm{~Hz}, 1 \mathrm{H}), 7.95-7.90(\mathrm{~m}, 1 \mathrm{H}), 7.82-7.75(\mathrm{~m}, 3 \mathrm{H}), 7.71-7.66(\mathrm{~m}, 2 \mathrm{H}), 4.92-$ $4.63(\mathrm{~m}, 2 \mathrm{H}), 4.27-4.22(\mathrm{~m}, 2 \mathrm{H})$.

${ }^{13}$ C NMR (100 MHz, $\left.\mathbf{C D C l}_{3}\right) \delta 168.2,155.9,144.2,134.9,134.1,132.5,131.9$, 128.4, 125.1, 123.4, 119.7, 48.6, 36.5.

HRMS (ESI-TOF) m/z: [M $+\mathrm{Na}]^{+}$Calcd for $\mathrm{C}_{17} \mathrm{H}_{12} \mathrm{~N}_{4} \mathrm{O}_{3} \mathrm{Na}$ 343.0807; Found 343.0806 .

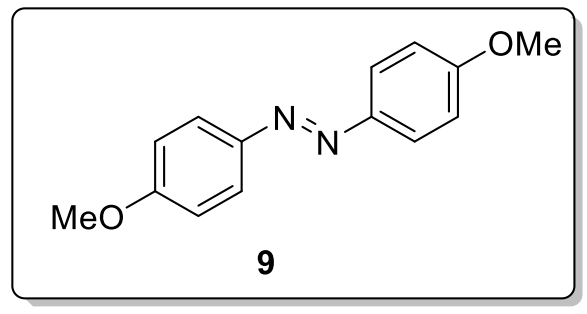

(E)-1,2-bis(4-methoxyphenyl)diazene 
9: Yellow solid, m. p.159.4 - $161.9^{\circ} \mathrm{C}(67 \mathrm{mg}, 55 \%$ yield on $1.0 \mathrm{mmol}$ scale)

TLC: $\mathrm{R}_{\mathrm{f}}=0.6($ Petroleum ether $/ \mathrm{EtOAc}=20 / 1)$

${ }^{1}$ H NMR (500 MHz, CDCl $) \delta 7.81(\mathrm{~d}, J=9.0 \mathrm{~Hz}, 4 \mathrm{H}), 6.93(\mathrm{~d}, J=9.0 \mathrm{~Hz}, 4 \mathrm{H})$, $3.81(\mathrm{~s}, 6 \mathrm{H})$.

${ }^{13}$ C NMR (125 MHz, CDCl $) \delta 160.5,146.0,123.3,113.1,54.5$.

HRMS (ESI-TOF) m/z: $[\mathrm{M}+\mathrm{Na}]^{+}$Calcd for $\mathrm{C}_{14} \mathrm{H}_{14} \mathrm{~N}_{2} \mathrm{O}_{2} \mathrm{Na}$ 265.0953; Found 265.0949.

\section{X-ray Crystallographic Data}<smiles>CC(C)(C)n1nnc2ccccc2c1=O</smiles>

$3 a$

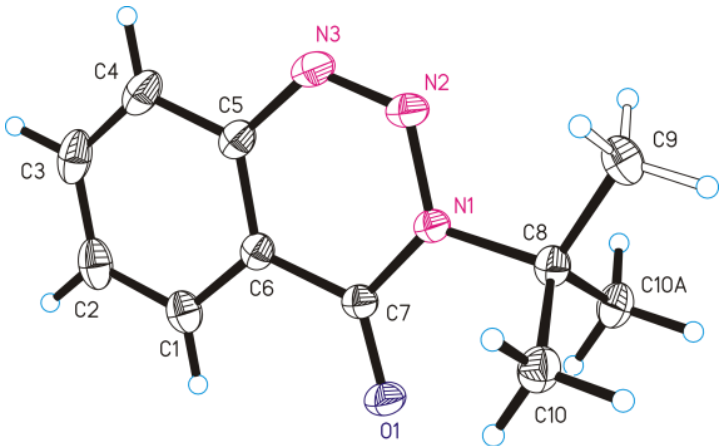

Figure S3. X-ray Crystallographic Data for Compound 3a<smiles>CC(C)(C)n1nnc2cnccc2c1=O</smiles>

$3 p$

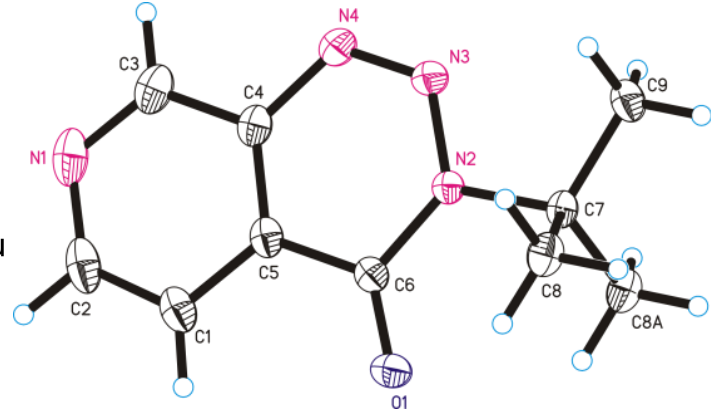

Figure S4. X-ray Crystallographic Data for Compound 3p<smiles>O=c1c2ccccc2nnn1C1CC1</smiles>

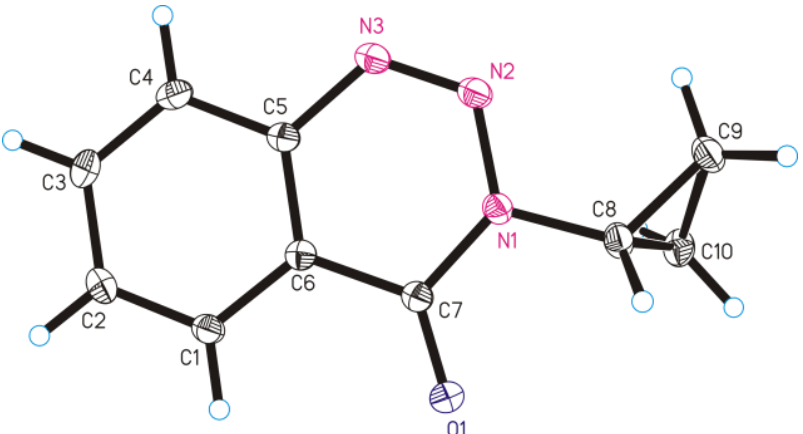

Figure S5. X-ray Crystallographic Data for Compound 3t 


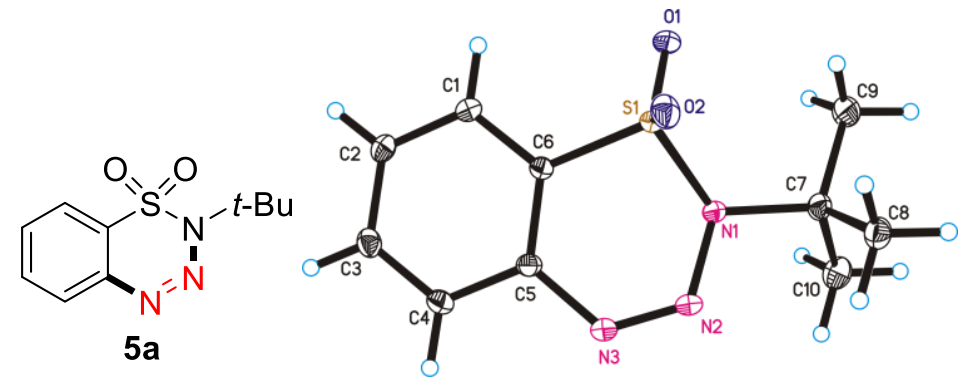

Figure S6. X-ray Crystallographic Data for Compound 5a

Table S1. Crystal data and structure refinements for 3a, 3p, 3t and 5a

\begin{tabular}{|c|c|c|c|c|}
\hline Compound & $\mathbf{3 a}$ & $3 p$ & $3 t$ & $5 \mathbf{a}$ \\
\hline CCDC code & 1959698 & 1972989 & 1969707 & 1972557 \\
\hline Empirical formula & $\mathrm{C}_{11} \mathrm{H}_{13} \mathrm{~N}_{3} \mathrm{O}$ & $\mathrm{C}_{10} \mathrm{H}_{12} \mathrm{~N}_{4} \mathrm{O}$ & $\mathrm{C}_{10} \mathrm{H}_{9} \mathrm{~N}_{3} \mathrm{O}$ & $\mathrm{C}_{10} \mathrm{H}_{13} \mathrm{~N}_{3} \mathrm{O}_{2} \mathrm{~S}$ \\
\hline Formula weight & 203.24 & 204.24 & 187.20 & 239.29 \\
\hline Crystal system & orthorhombic & monoclinic & monoclinic & monoclinic \\
\hline Space group & $\mathrm{Pn} \mathrm{ma}$ & $\mathrm{P} 121 / \mathrm{m} 1$ & P 1211 & P $121 / n$ \\
\hline Hall group & $-\mathrm{P} 2 \mathrm{ac} 2 \mathrm{n}$ & $-P 2 y b$ & $\mathrm{P} 2 \mathrm{yb}$ & $-\mathrm{P} 2 \mathrm{yn}$ \\
\hline $\mathrm{a} / \mathrm{A}$ & $8.785(3)$ & $10.660(5)$ & $5.1731(16)$ & $7.390(3)$ \\
\hline $\mathrm{b} / \AA$ & $6.828(2)$ & $6.728(3)$ & $15.782(7)$ & $7.882(3)$ \\
\hline c/Å & $17.553(7)$ & $14.479(6)$ & $5.2846(17)$ & $19.510(9)$ \\
\hline$\alpha /^{\circ}$ & 90 & 90 & 90 & 90 \\
\hline$\beta /{ }^{\circ}$ & 90 & $93.675(17)$ & $92.440(16)$ & $97.958(18)$ \\
\hline$\gamma /{ }^{\circ}$ & 90 & 90 & 90 & 90 \\
\hline Volume & $1052.8(6)$ & $1036.4(9)$ & $431.1(3)$ & $1125.5(8)$ \\
\hline $\mathrm{Z}$ & 4 & 4 & 2 & 4 \\
\hline Temperature/K & $170 \mathrm{~K}$ & $170(2)$ & $170(2)$ & $170(2)$ \\
\hline X-ray wavelength/Å & 0.71073 & 0.71073 & 0.71073 & 0.71073 \\
\hline $2 \theta$ range for data collection $/^{\circ}$ & 3.29 to 27.08 & 2.45 to 27.09 & 2.58 to 27.10 & 2.80 to 27.13 \\
\hline Crystal color & colourless & colourless & colourless & colourless \\
\hline$\rho$ Calcd $g / \mathrm{m}^{3}$ & 1.282 & 1.309 & 1.442 & 1.412 \\
\hline$\mu / \mathrm{mm}^{-1}$ & 0.086 & 0.090 & 0.098 & 0.277 \\
\hline Max. transmission & 0.7455 & 0.7455 & 0.7455 & 0.7455 \\
\hline Min. transmission & 0.6982 & 0.6918 & 0.6973 & 0.6412 \\
\hline F_000 & 432 & 432 & 196 & 504 \\
\hline Crystal_size $/ \mathrm{mm}^{3}$ & $0.26 \times 0.16 \times 0.12$ & $0.38 \times 0.13 \times 0.08$ & $0.49 \times 0.42 \times 0.23$ & $0.39 \times 0.28 \times 0.26$ \\
\hline Radiation & MoKla & MoKla & MoKla & MoKla \\
\hline Independent reflections $\mathrm{I}>2 \sigma$ & 1109 & 1943 & 1758 & 2303 \\
\hline
\end{tabular}




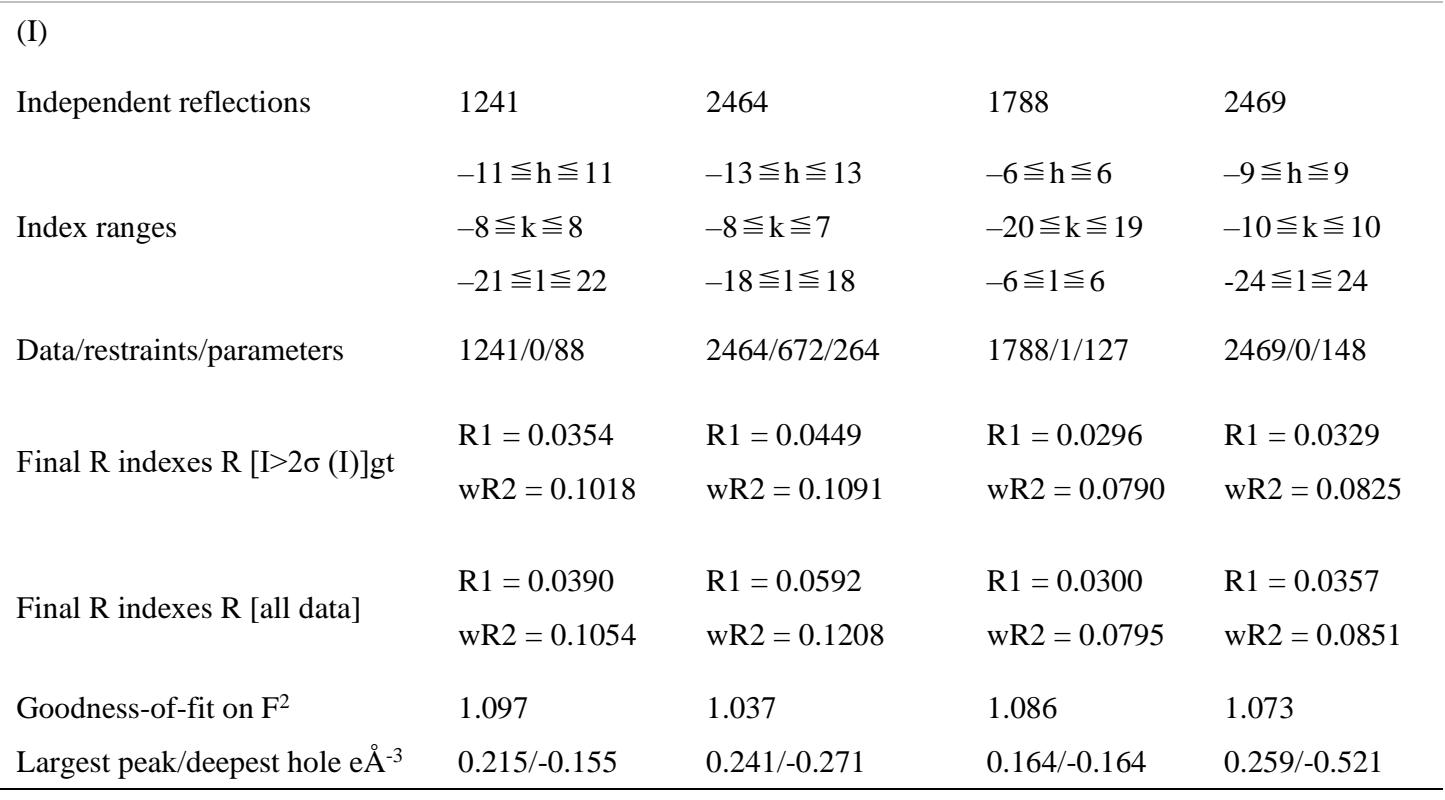

\section{References}

1. Guimond, N.; Gorelsky, S. I.; Fagnou, K., Rhodium(III)-catalyzed heterocycle synthesis using an internal oxidant: improved reactivity and mechanistic studies. J. Am. Chem. Soc. 2011, 133 (16), 6449-6457.

2. Shi, Y.; Zhang, L.; Lan, J.; Zhang, M.; Zhou, F.; Wei, W.; You, J., Oxidative C-H/C-H Cross-Coupling Reactions between N-Acylanilines and Benzamides Enabled by a $\mathrm{Cp}^{*}$-Free $\mathrm{RhCl}_{3} / \mathrm{TFA}$ Catalytic System. Angew. Chem. Int. Ed. 2018, 57 (29), 9108-9112.

3 .Chen, T.-T.; Wang, A.-E.; Huang, P.-Q., Chemoselective Synthesis of $\alpha$-Amino- $\alpha$-cyanophosphonates by Reductive Gem-Cyanation-Phosphonylation of Secondary Amides. Org. Lett. 2019, 21 (10), 3808-3812.

4. Xie, Y.; Pan, H.; Xiao, X.; Li, S.; Shi, Y., Organocatalytic asymmetric biomimetic transamination of aromatic ketone to optically active amine. Org. Biomo. Chem. 2012, 10 (45), 8960-8962.

5. Wang, G.-W.; Yuan, T.-T.; Li, D.-D., One-Pot Formation of $\mathrm{C}-\mathrm{C}$ and $\mathrm{C}-\mathrm{N}$ Bonds through Palladium-Catalyzed Dual C-H Activation: Synthesis of Phenanthridinones. Angew. Chem. Int. Ed. 2011, 50 (6), 1380-1383.

6. Meyer, D.; Jangra, H.; Walther, F.; Zipse, H.; Renaud, P., A third generation of radical fluorinating agents based on $N$-fluoro- $N$-arylsulfonamides. Nat. Commun. 2018, 9 (1), 4888-4897.

7. Griebenow, N.; Meyer, T., Solid-Phase Synthesis of 1,2-Diketones via Acetylene Oxidation: A Versatile Diversity Platform for the Combinatorial Synthesis of Heterocycles. Synlett 2010, 2010 (17), 2639-2643.

8. Ochiai, H.; Uetake, Y.; Niwa, T.; Hosoya, T., Rhodium-Catalyzed Decarbonylative Borylation of Aromatic Thioesters for Facile Diversification of Aromatic Carboxylic Acids. Angew. Chem. Int. Ed. 2017, 56 (9), 2482-2486.

9. Jones, M. W.; Mantovani, G.; Blindauer, C. A.; Ryan, S. M.; Wang, X.; Brayden, D. J.; Haddleton, D. M., Direct peptide bioconjugation/PEGylation at tyrosine with linear and branched polymeric diazonium salts. J. Am. Chem. Soc. 2012, 134 (17), 7406-7413. 


\section{Copies of NMR Spectra}

${ }^{1} \mathrm{H}$ - NMR spectrum of compound $\mathbf{3 a}\left(500 \mathrm{MHz}, \mathrm{CDCl}_{3}\right)$

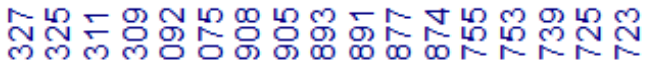

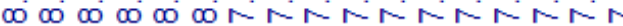

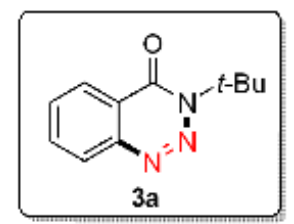

\section{ilit}

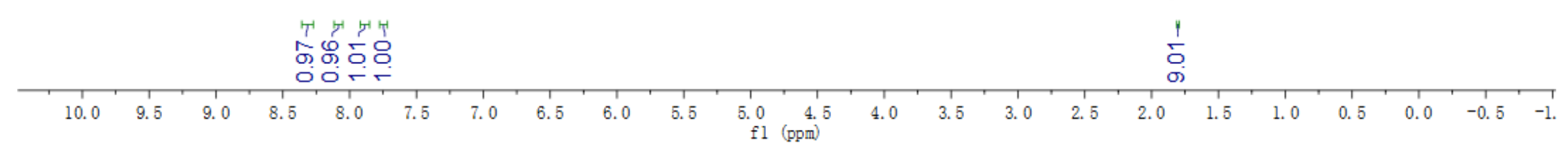


${ }^{13} \mathrm{C}$ - NMR spectrum of compound $\mathbf{3 a}\left(100 \mathrm{MHz}, \mathrm{CDCl}_{3}\right)$

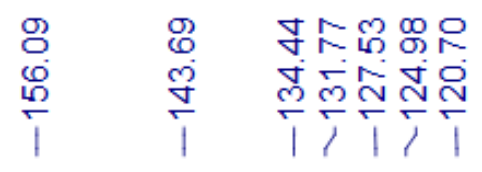

क)

م.
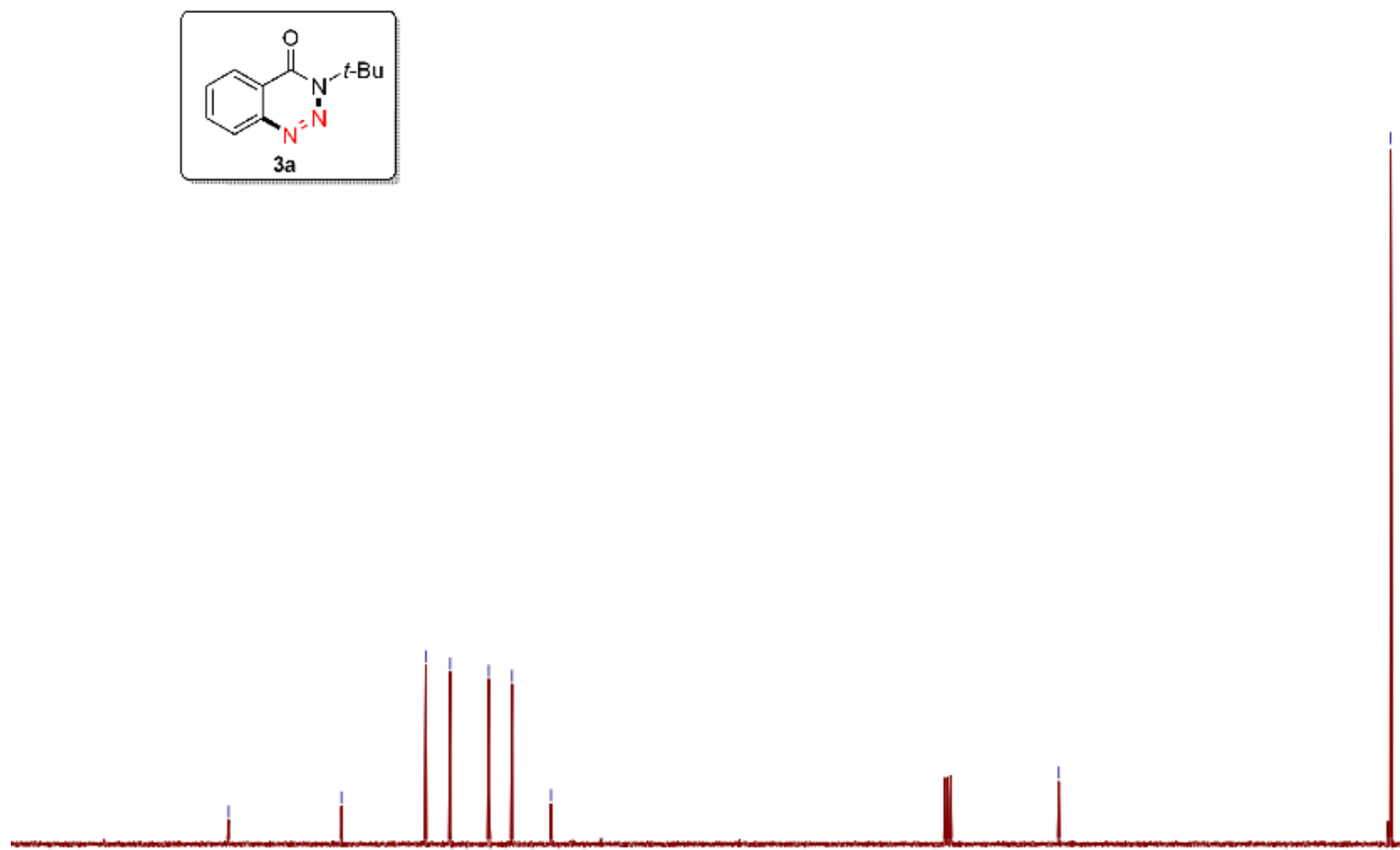
${ }^{1} \mathrm{H}$ - NMR spectrum of compound $\mathbf{3 b}\left(400 \mathrm{MHz}, \mathrm{CDCl}_{3}\right)$

ल)

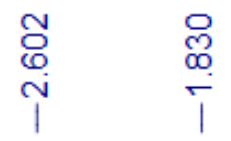
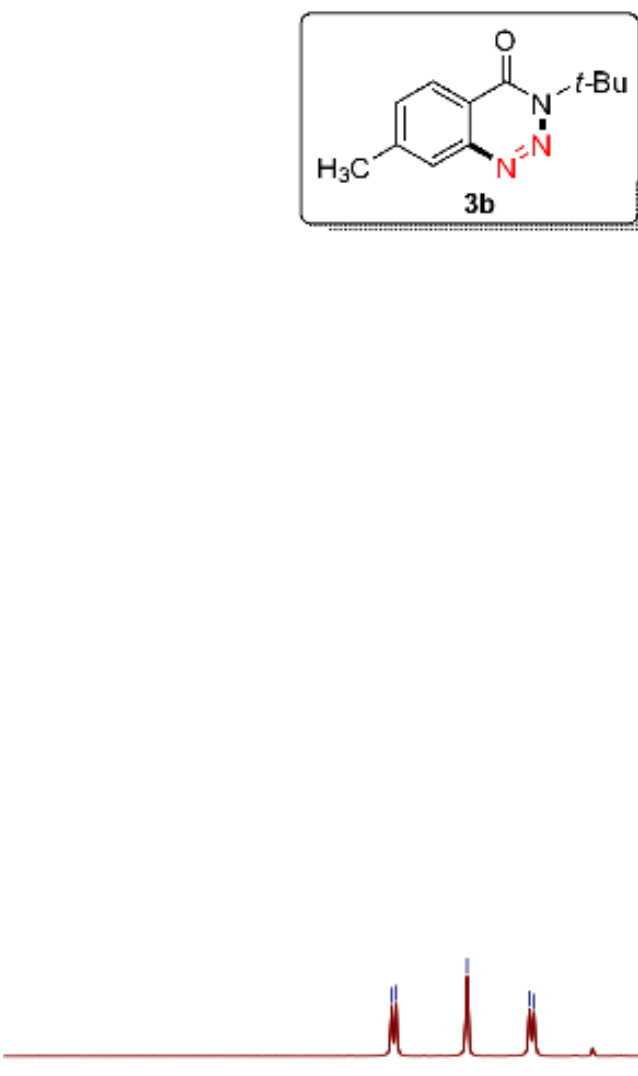

웅

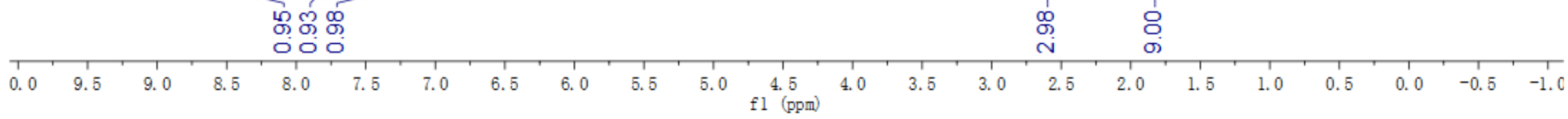


${ }^{13} \mathrm{C}$ - NMR spectrum of compound $\mathbf{3 b}\left(100 \mathrm{MHz}, \mathrm{CDCl}_{3}\right)$
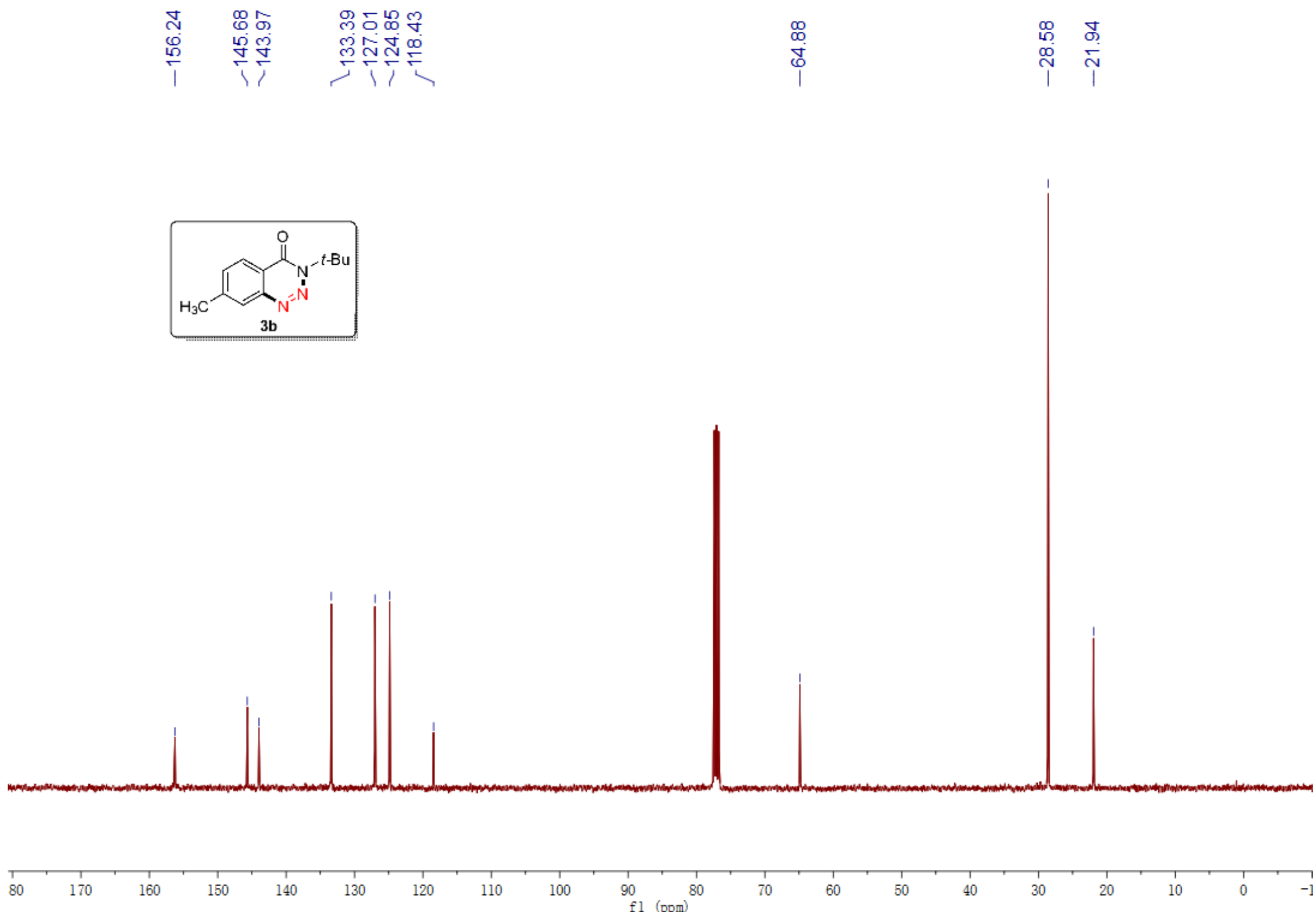
${ }^{1} \mathrm{H}$ - NMR spectrum of compound $3 \mathbf{c}\left(600 \mathrm{MHz}, \mathrm{CDCl}_{3}\right)$

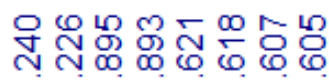

$\infty 0 m-1$

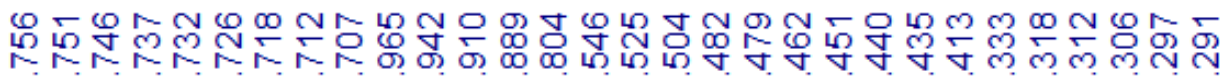

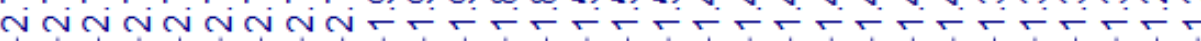
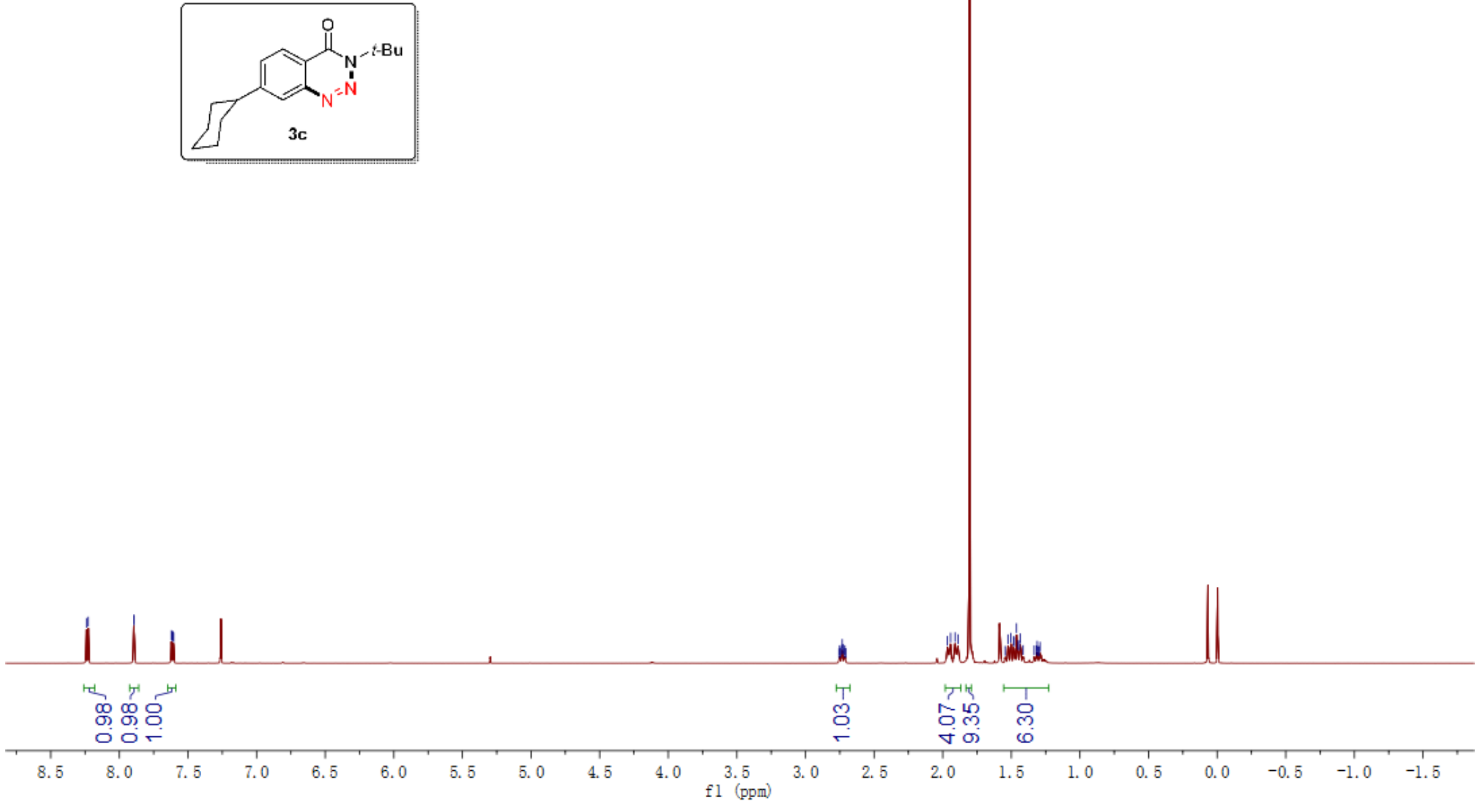
${ }^{13} \mathrm{C}$ - NMR spectrum of compound $3 \mathbf{c}\left(100 \mathrm{MHz}, \mathrm{CDCl}_{3}\right)$

$$
\text { 沜 }
$$
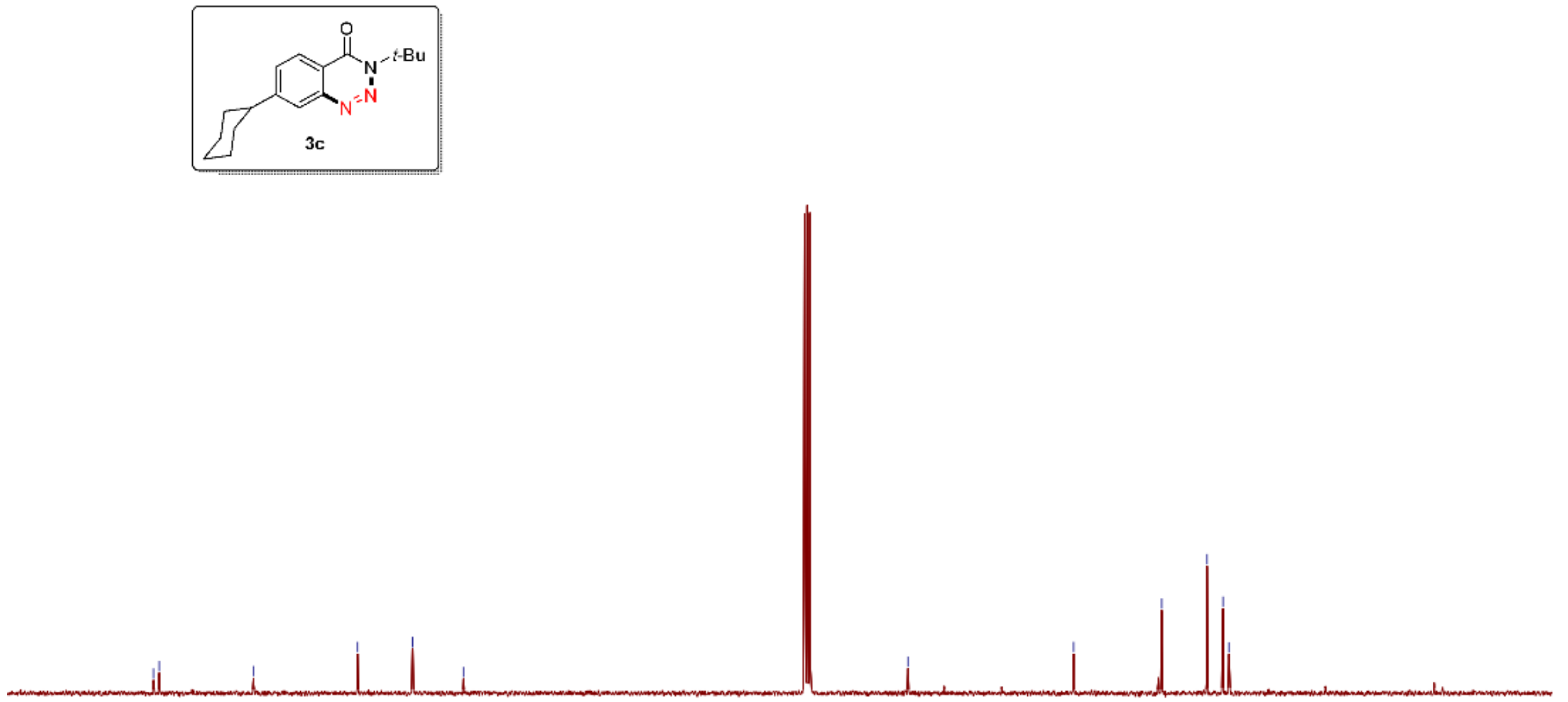
${ }^{1} \mathrm{H}$ - NMR spectrum of compound $\mathbf{3 d}\left(600 \mathrm{MHz}, \mathrm{CDCl}_{3}\right)$

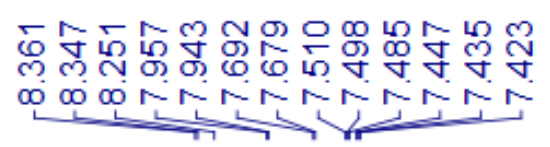

$\underset{\infty}{\infty}$

i
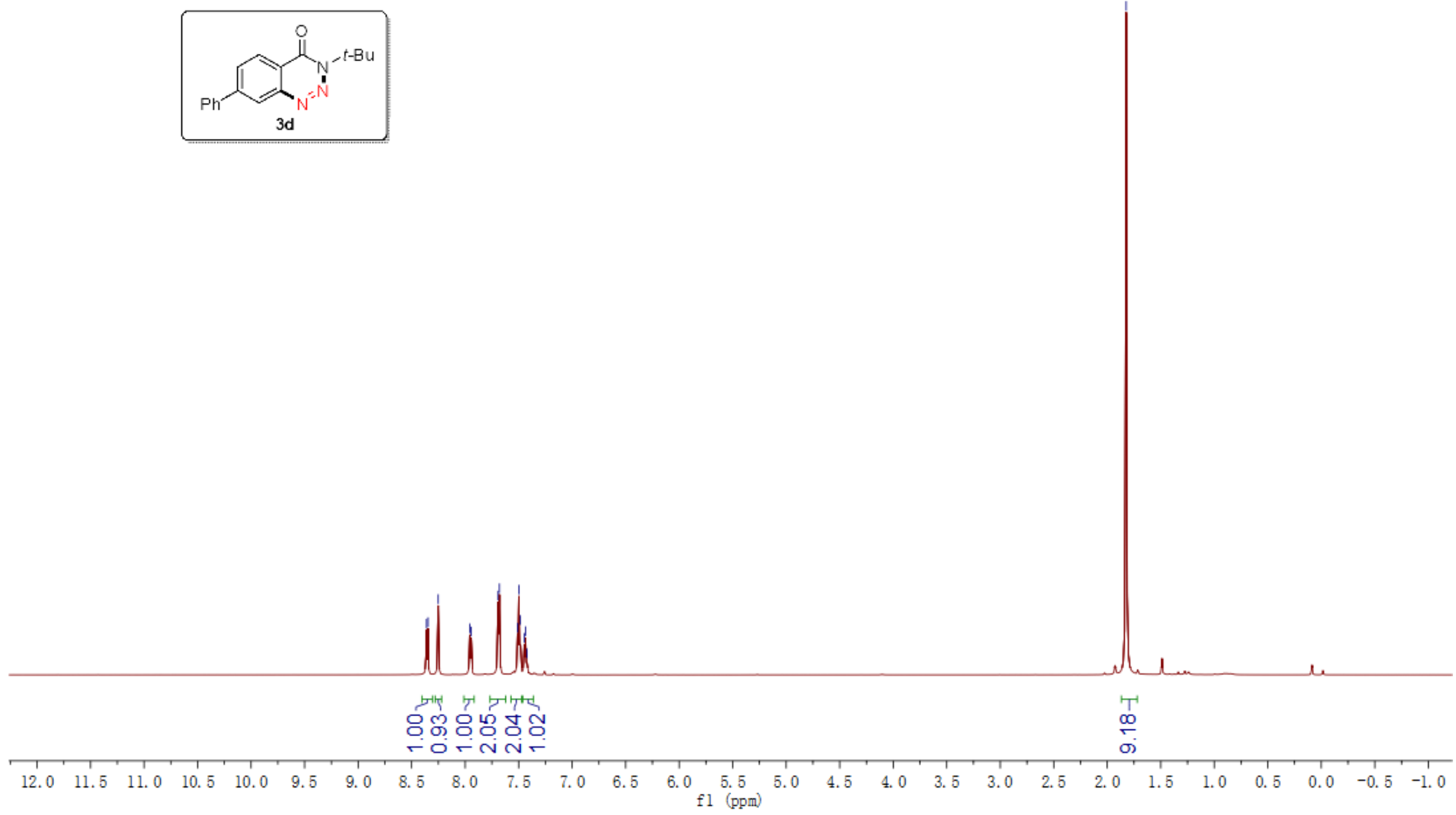
${ }^{13} \mathrm{C}-\mathrm{NMR}$ spectrum of compound $3 \mathbf{d}\left(100 \mathrm{MHz}, \mathrm{CDCl}_{3}\right)$

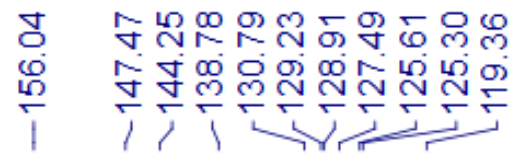

$\underset{\substack{0 \\ 0}}{1}$

ำ

ind
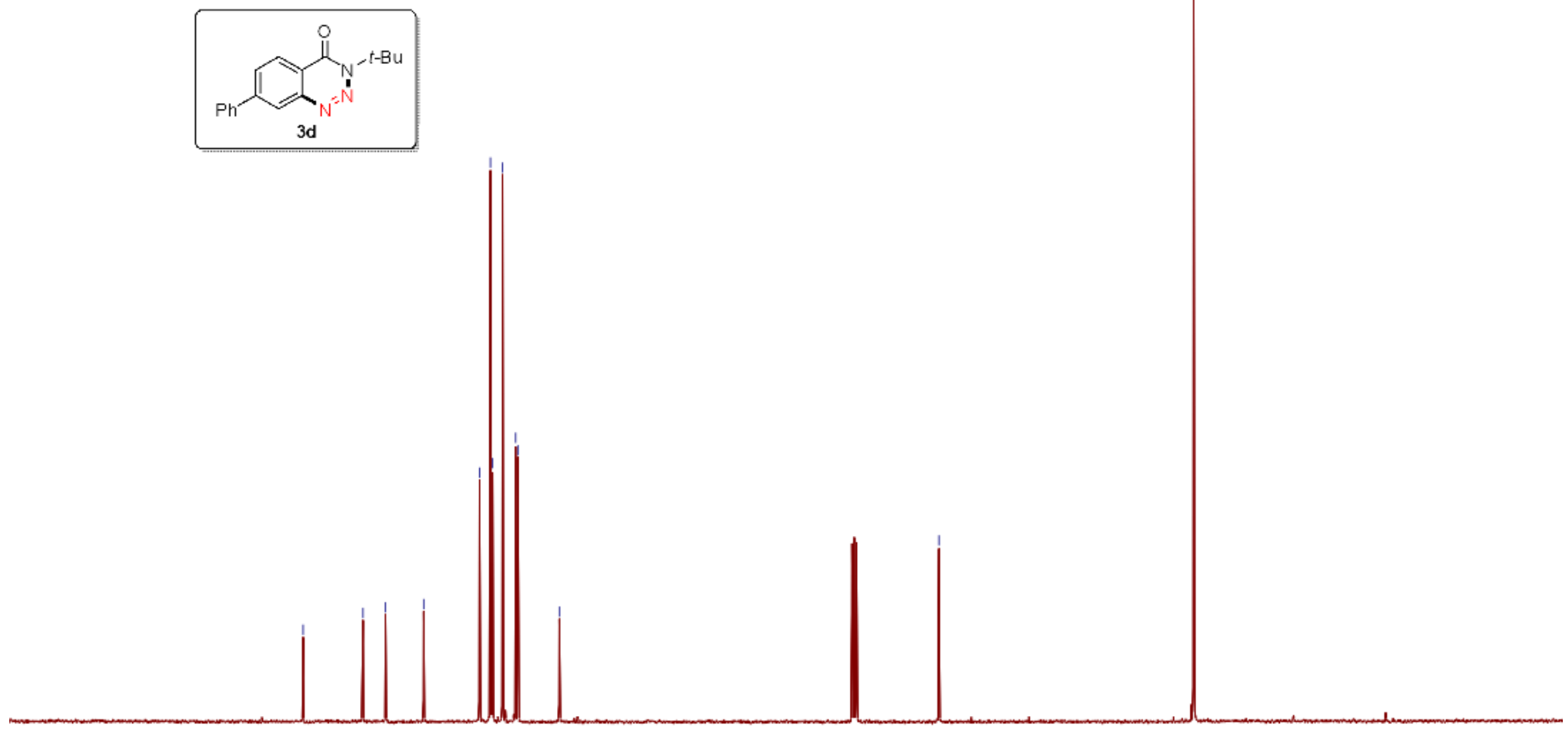

(1) 
${ }^{1} \mathrm{H}$ - NMR spectrum of compound $\mathbf{3 e}\left(400 \mathrm{MHz}, \mathrm{CDCl}_{3}\right)$
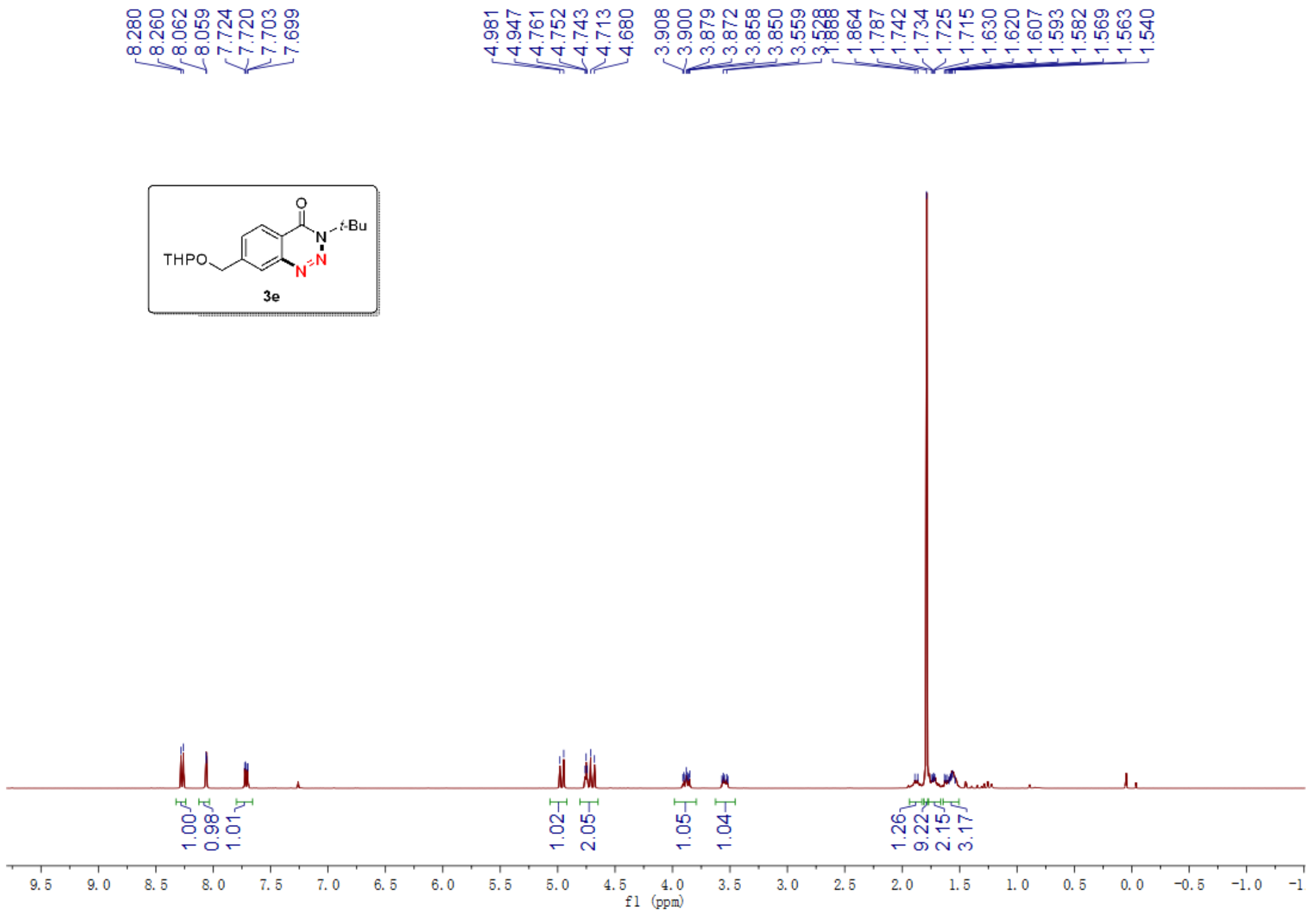

S41 
${ }^{13} \mathrm{C}$ - NMR spectrum of compound $3 \mathbf{e}\left(100 \mathrm{MHz}, \mathrm{CDCl}_{3}\right)$

\begin{tabular}{|c|c|c|c|c|}
\hline 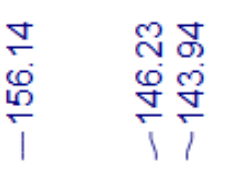 & 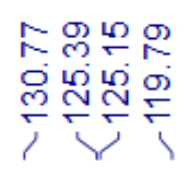 & $\begin{array}{l}0 \\
\text { m. } \\
\infty \\
\infty \\
1\end{array}$ & 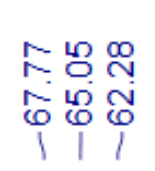 & 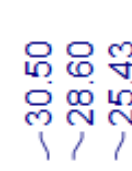 \\
\hline
\end{tabular}
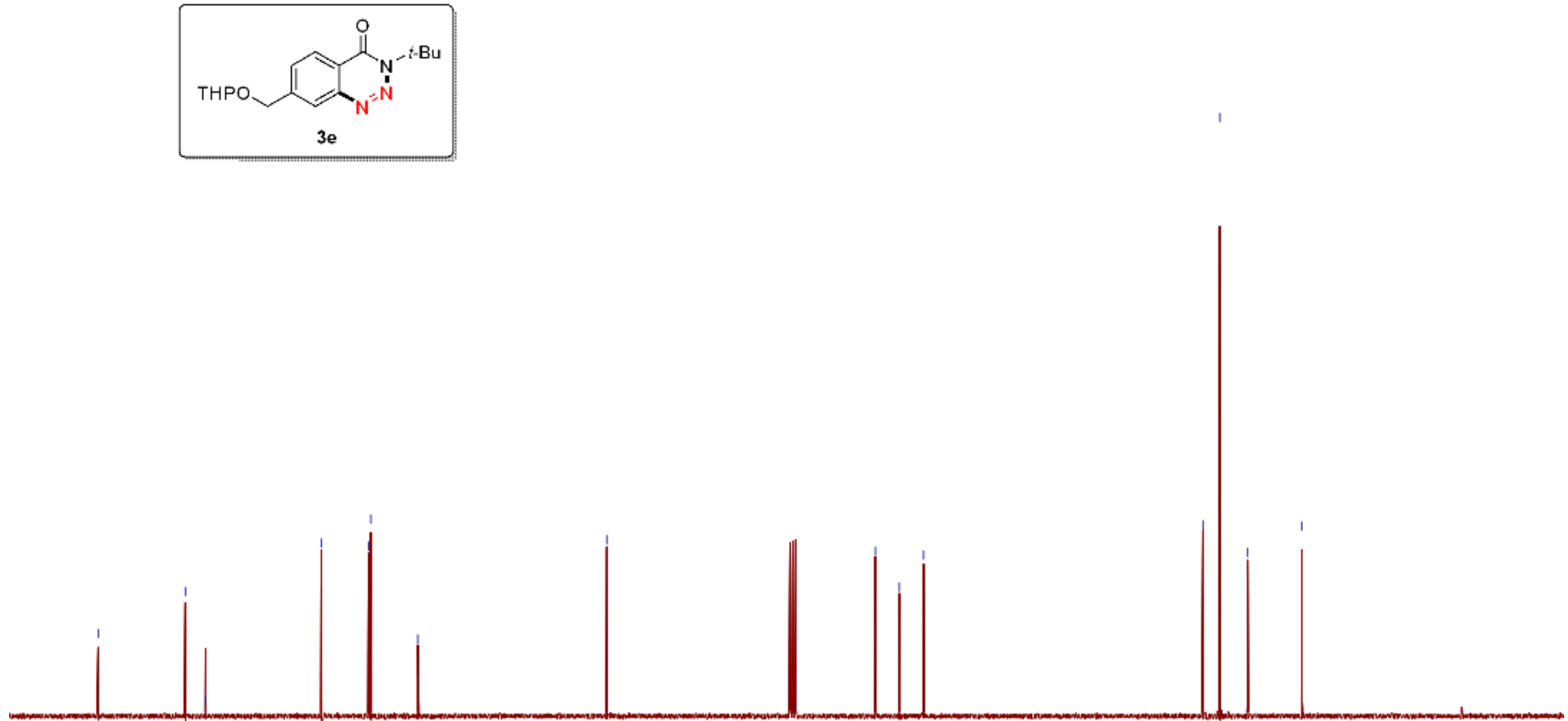
${ }^{1} \mathrm{H}$ - NMR spectrum of compound $\mathbf{3 f}\left(400 \mathrm{MHz}, \mathrm{CDCl}_{3}\right)$

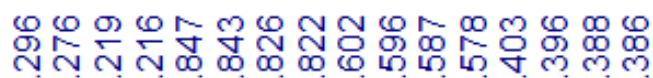
$\infty \infty \infty \infty \cdots+\cdots$
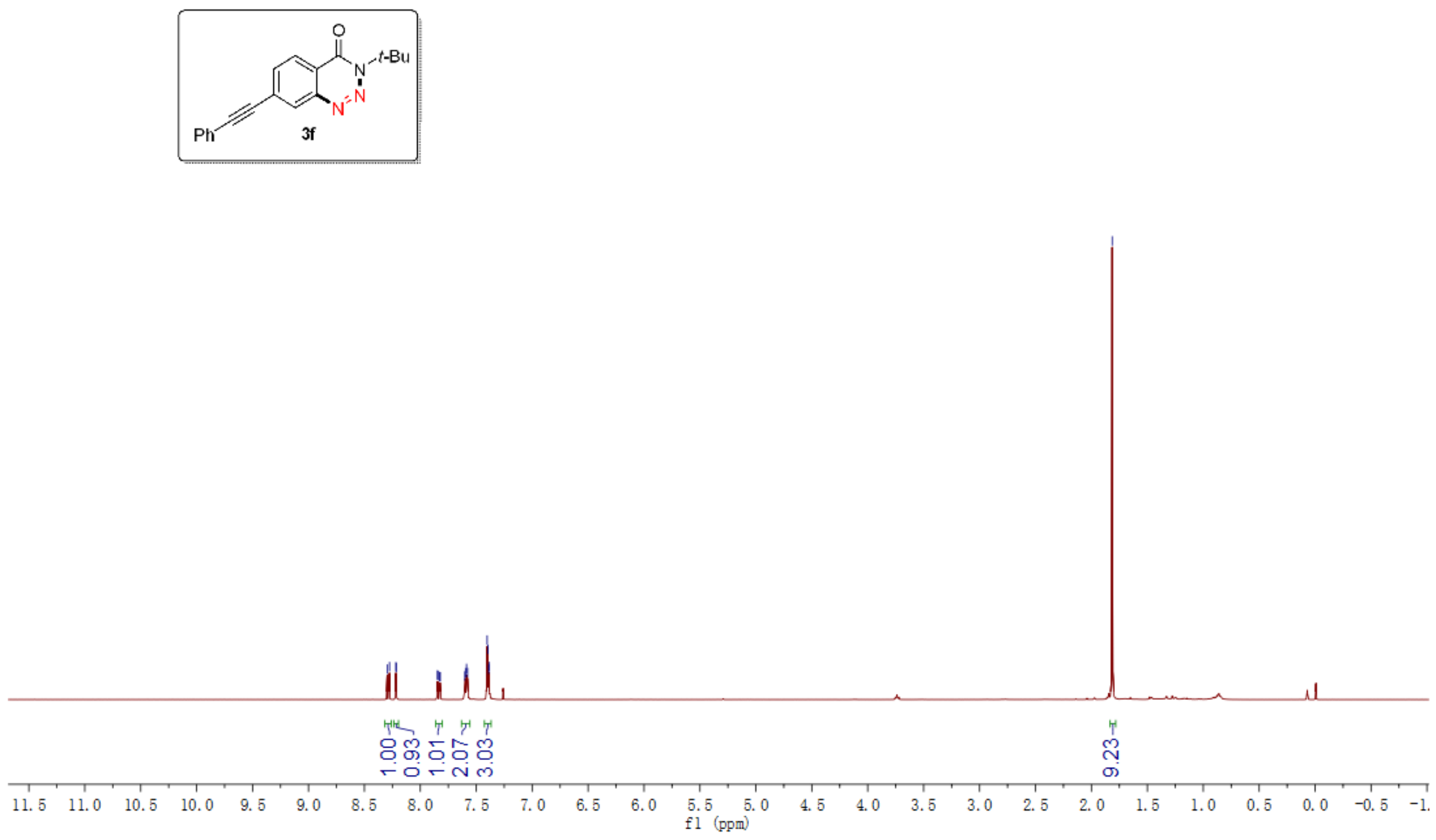
${ }^{13} \mathrm{C}$ - NMR spectrum of compound $3 f\left(100 \mathrm{MHz}, \mathrm{CDCl}_{3}\right)$

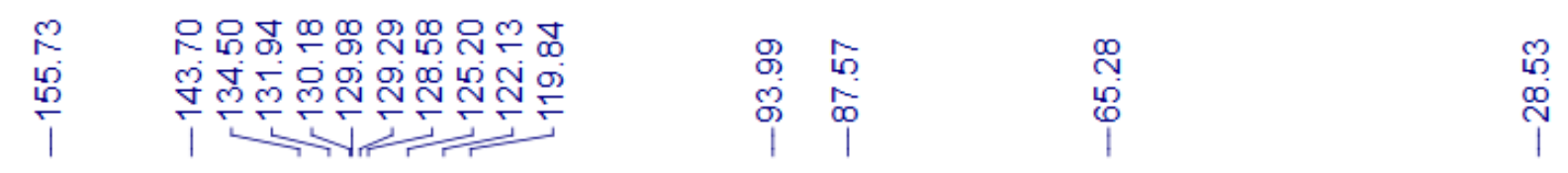
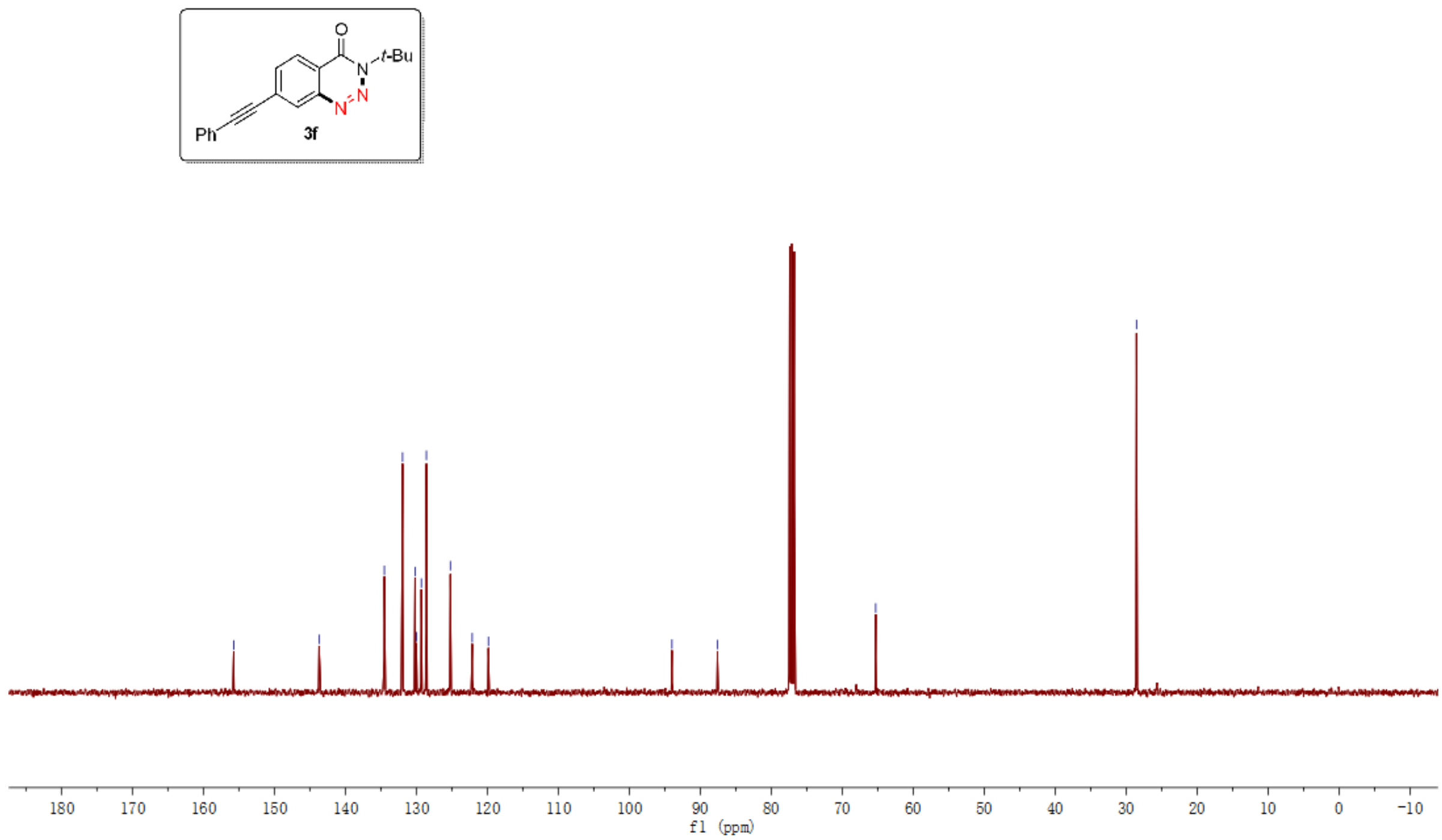
${ }^{1} \mathrm{H}$ - NMR spectrum of compound $\mathbf{3 g}\left(500 \mathrm{MHz}, \mathrm{CDCl}_{3}\right)$

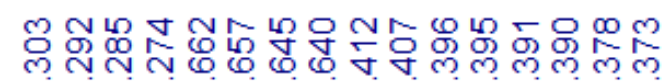

$\infty \infty \infty \infty \cdots+1$
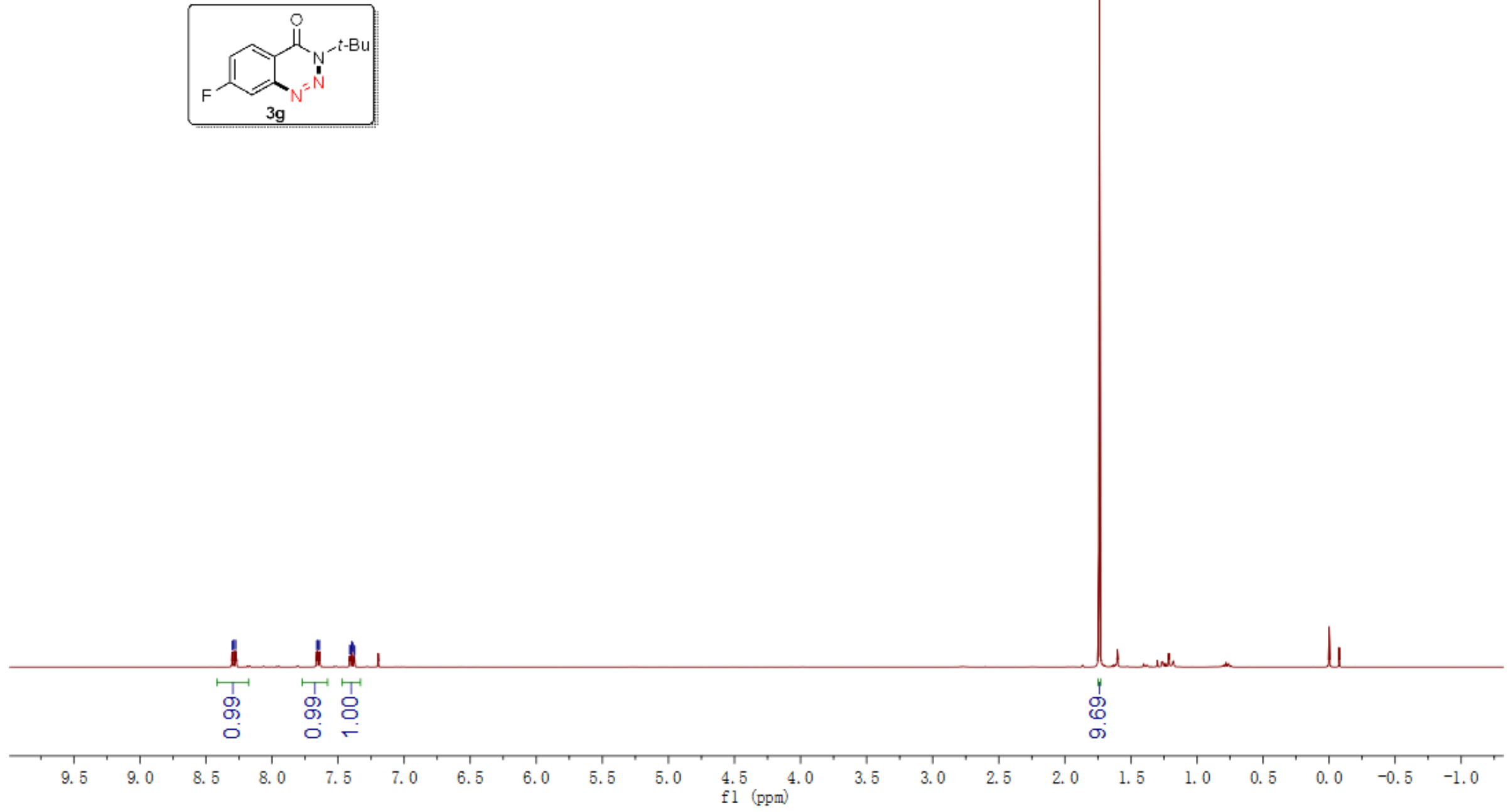
${ }^{13} \mathrm{C}$ - NMR spectrum of compound $\mathbf{3 g}\left(125 \mathrm{MHz}, \mathrm{CDCl}_{3}\right)$

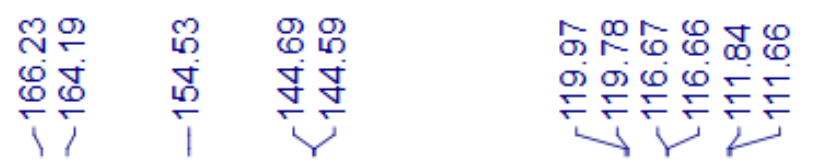
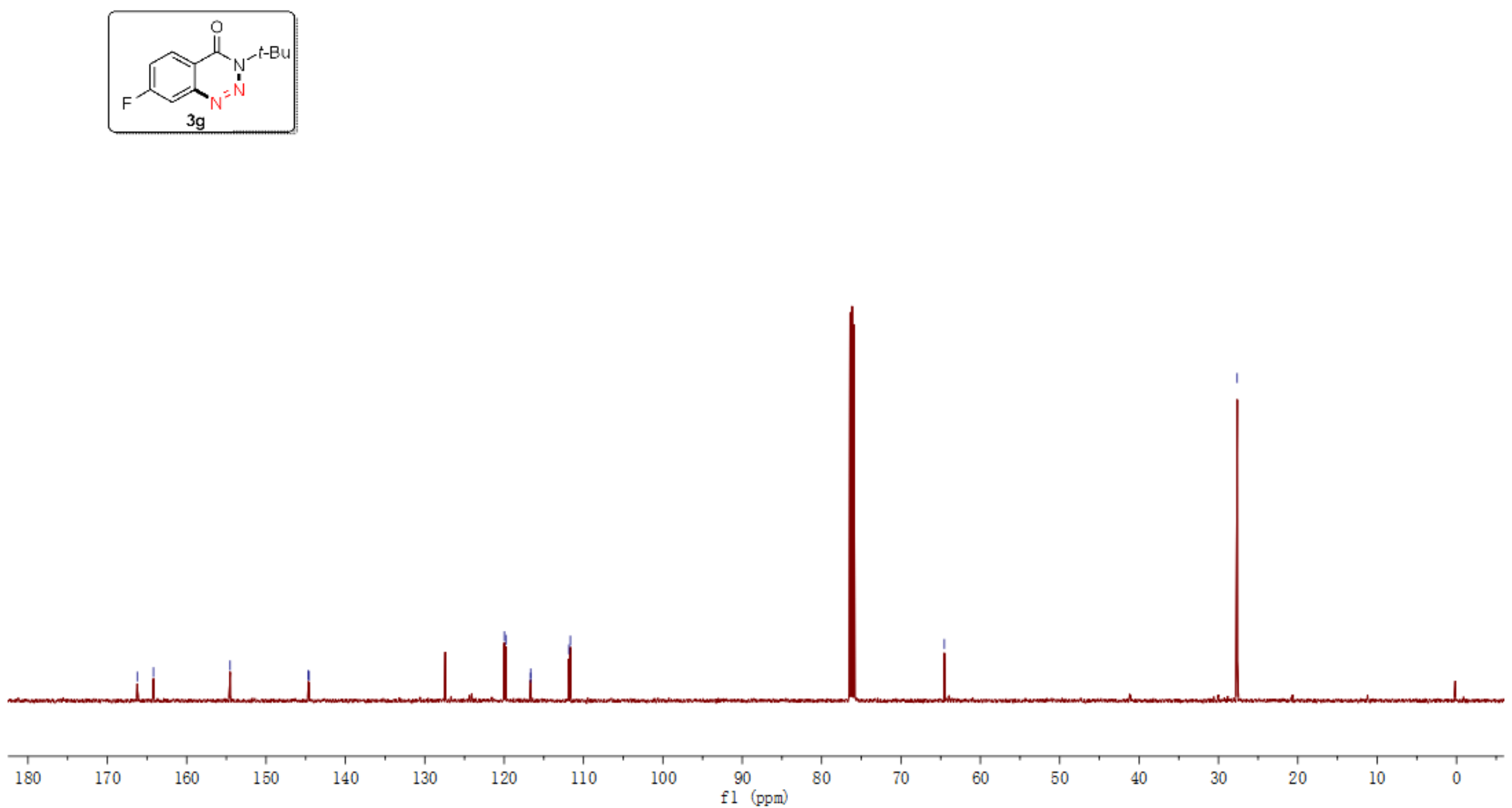
${ }^{1} \mathrm{H}$ - NMR spectrum of compound $\mathbf{3 h}\left(500 \mathrm{MHz}, \mathrm{CDCl}_{3}\right)$

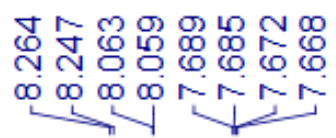

$\stackrel{\leftrightarrow}{\stackrel{\circ}{\circ}}$
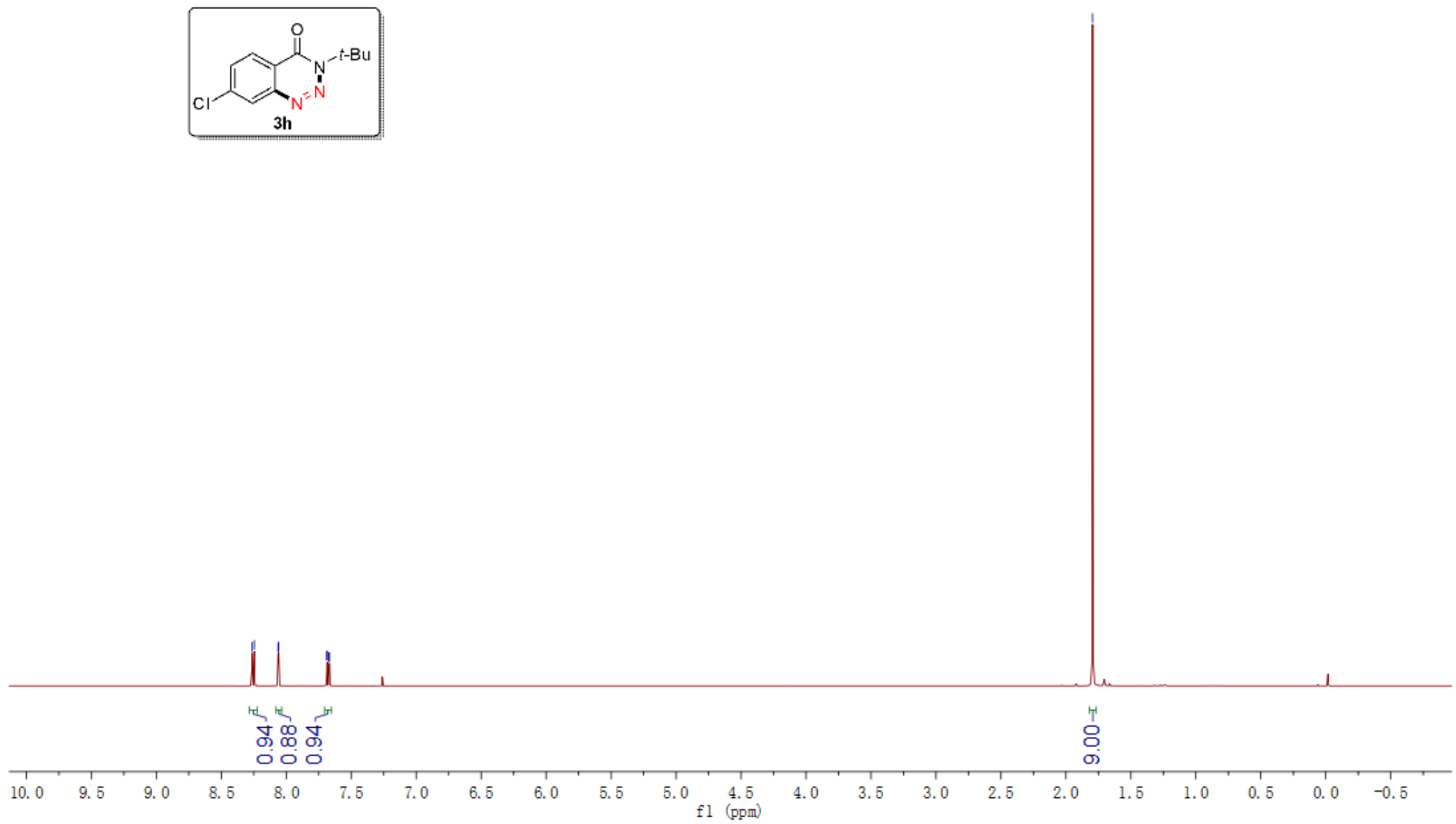
${ }^{13} \mathrm{C}$ - NMR spectrum of compound $\mathbf{3 h}\left(125 \mathrm{MHz}, \mathrm{CDCl}_{3}\right)$

咅

0
0
0
0
1

$\stackrel{\infty}{q}$
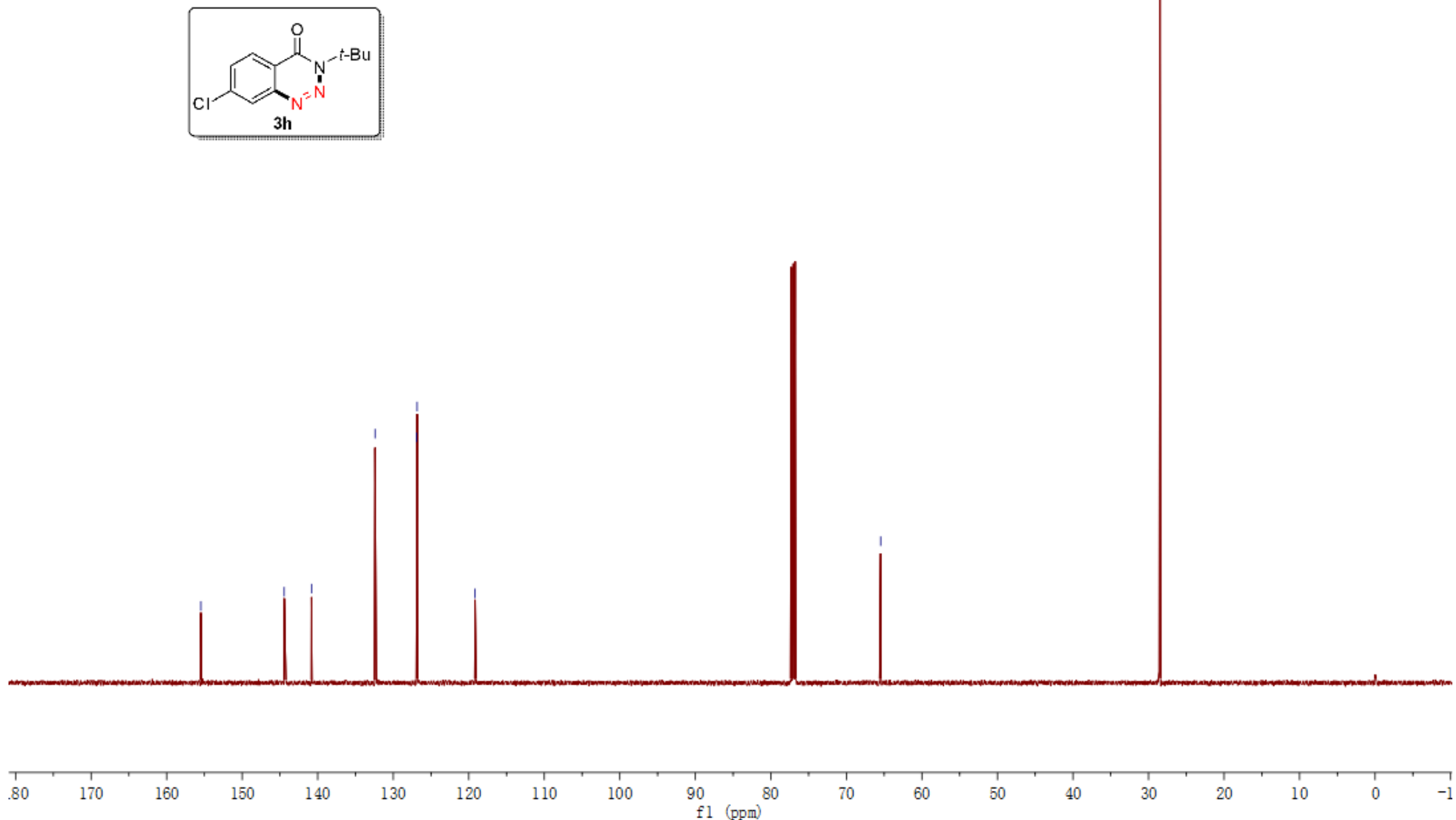
${ }^{1} \mathrm{H}$ - NMR spectrum of compound $3 \mathbf{i}\left(400 \mathrm{MHz}, \mathrm{CDCl}_{3}\right)$

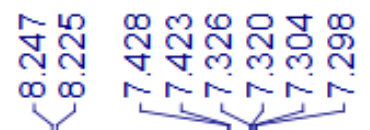

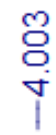

No
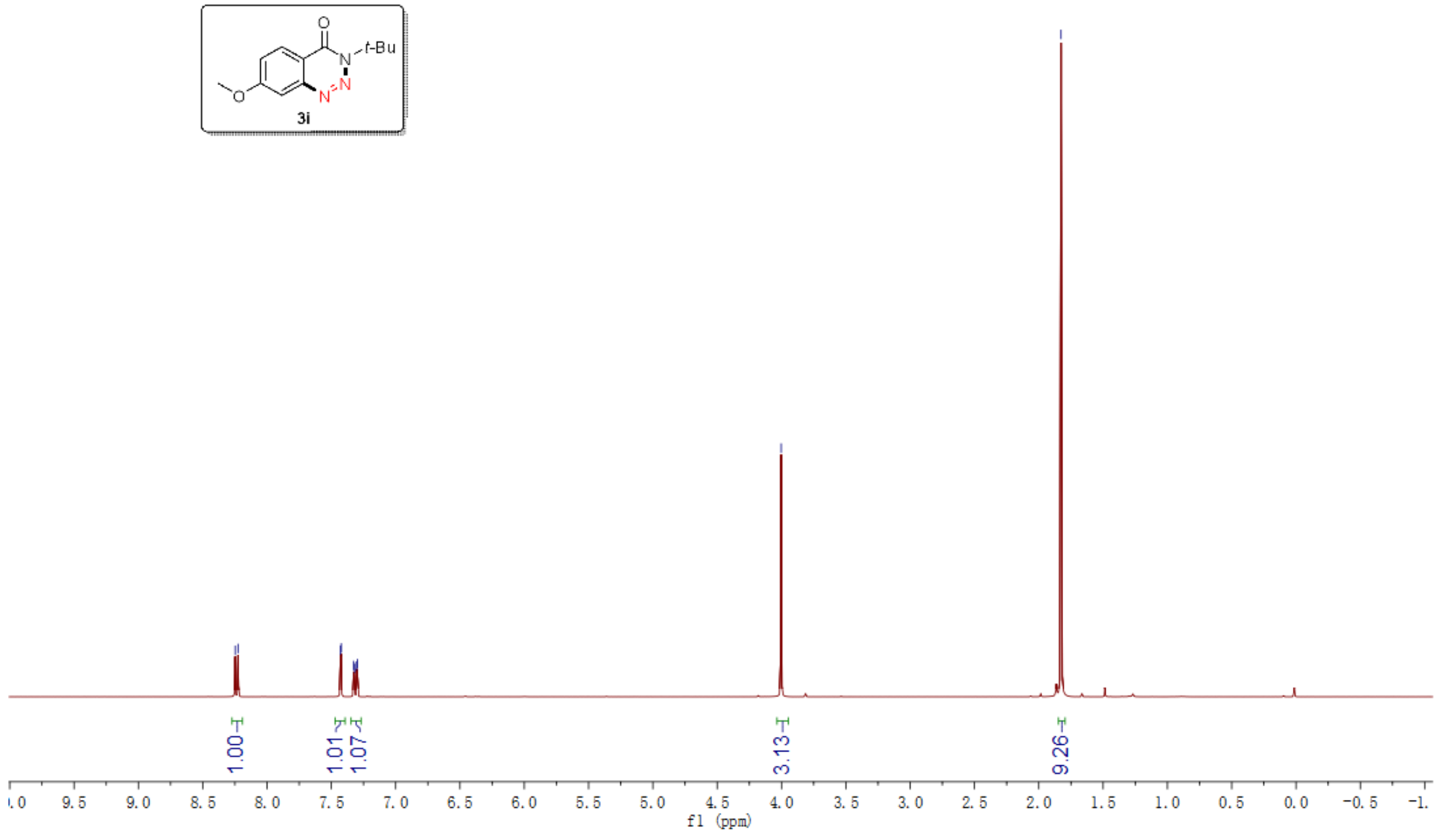
${ }^{13} \mathrm{C}$ - NMR spectrum of compound $3 \mathbf{i}\left(100 \mathrm{MHz}, \mathrm{CDCl}_{3}\right)$

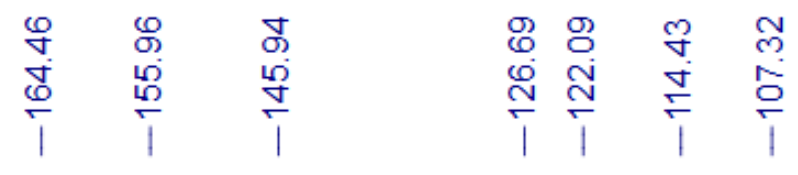
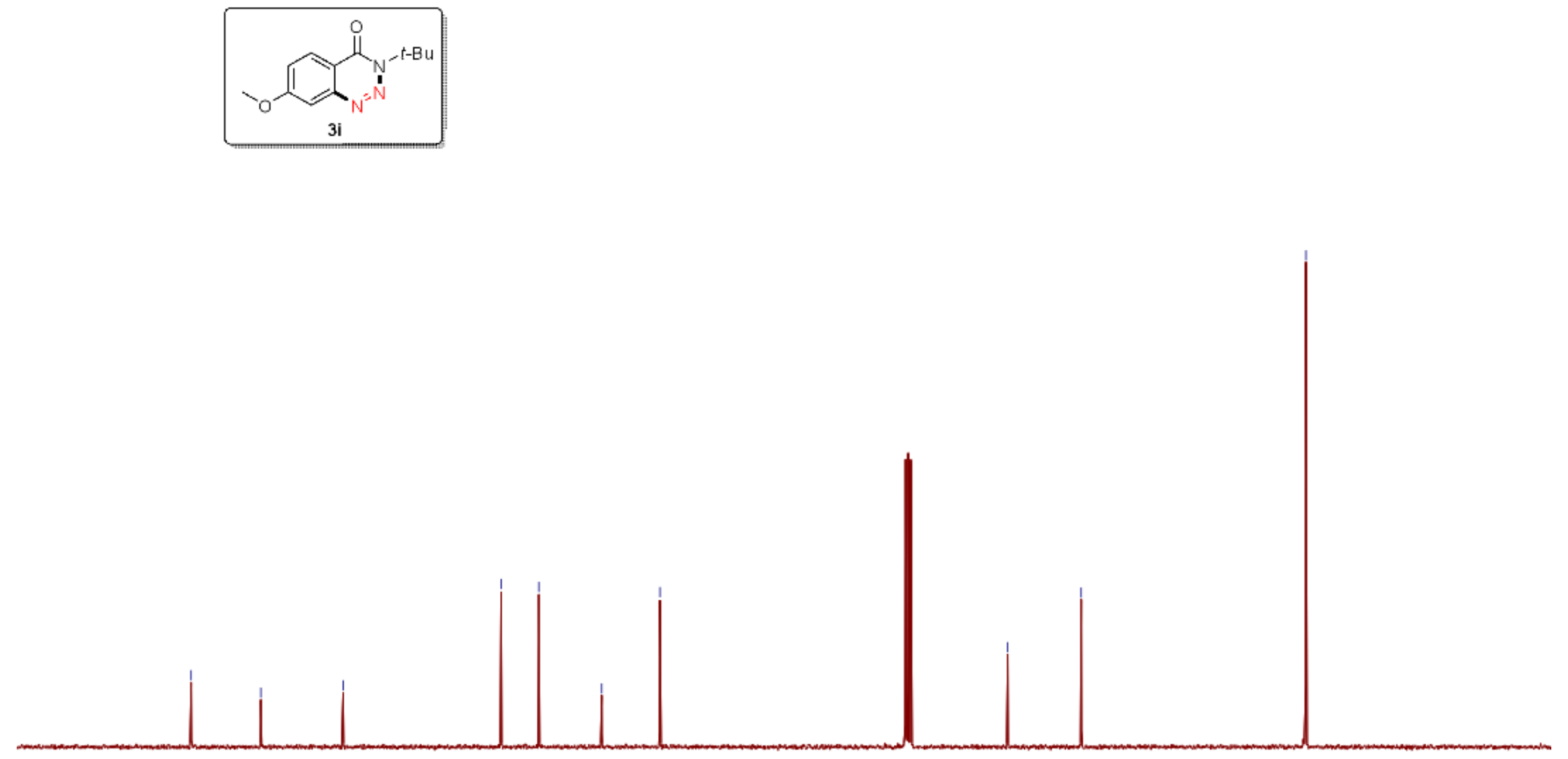
${ }^{1} \mathrm{H}$ - NMR spectrum of compound $\mathbf{3 j}$ (400 MHz, $\mathrm{CDCl}_{3}$ )

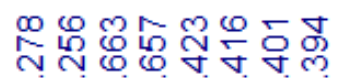

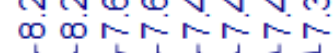
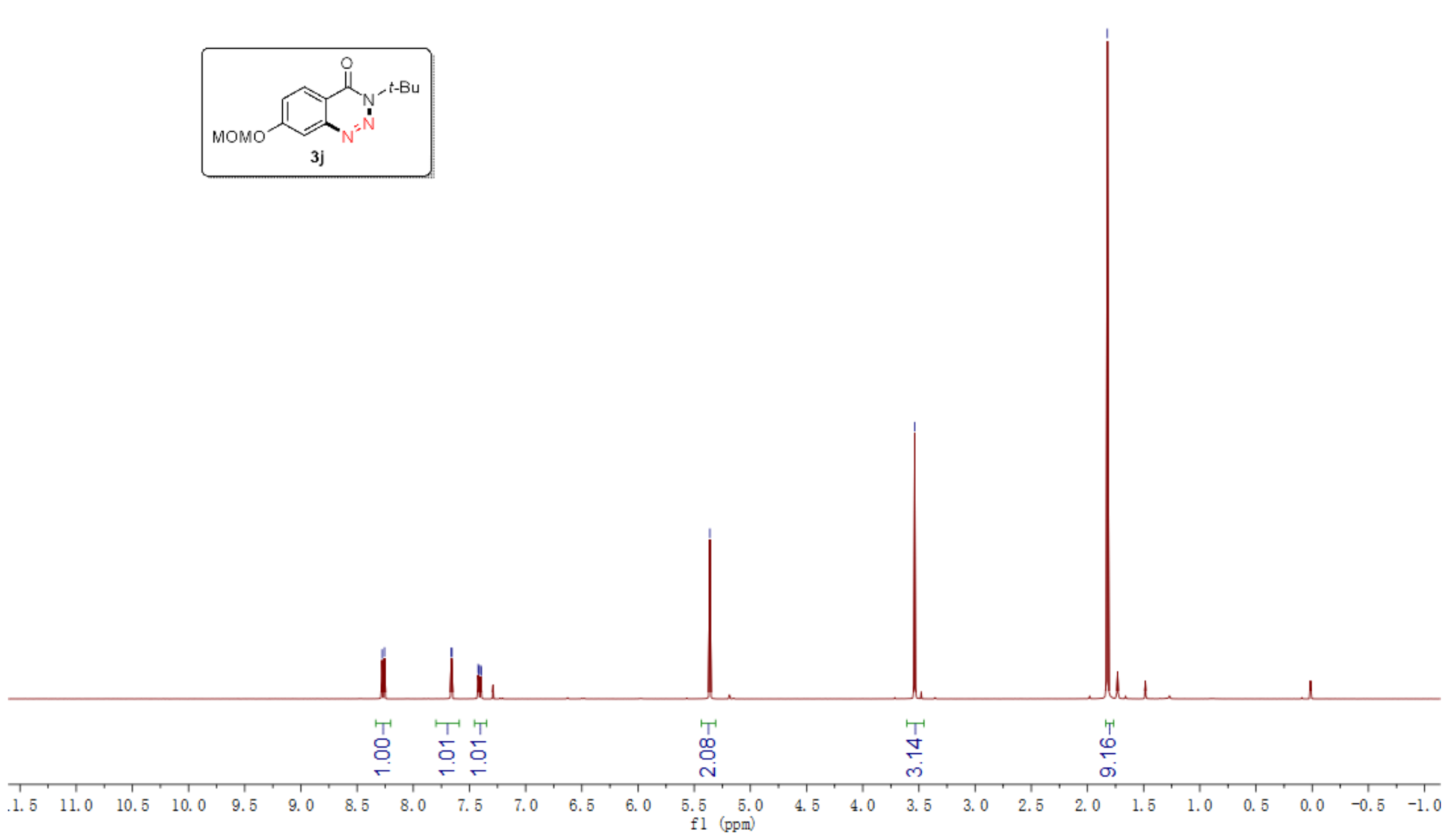
${ }^{13} \mathrm{C}$ - NMR spectrum of compound $\mathbf{3 j}\left(100 \mathrm{MHz}, \mathrm{CDCl}_{3}\right)$

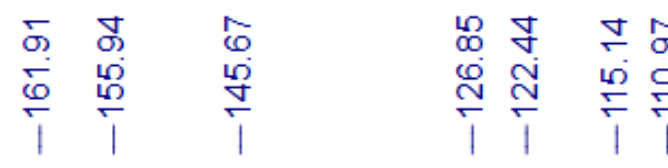

$\underset{\substack{\text { P } \\ \text { i }}}{\mathfrak{m}}$

क.

!

$\bar{\omega}$



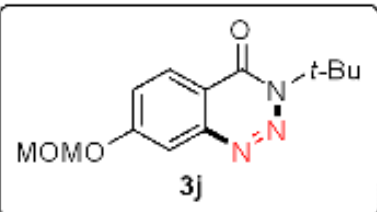

3j

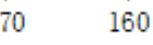

150 
${ }^{1} \mathrm{H}$ - NMR spectrum of compound $\mathbf{3 k}\left(400 \mathrm{MHz}, \mathrm{CDCl}_{3}\right.$ )

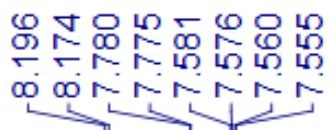

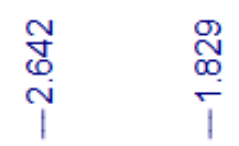
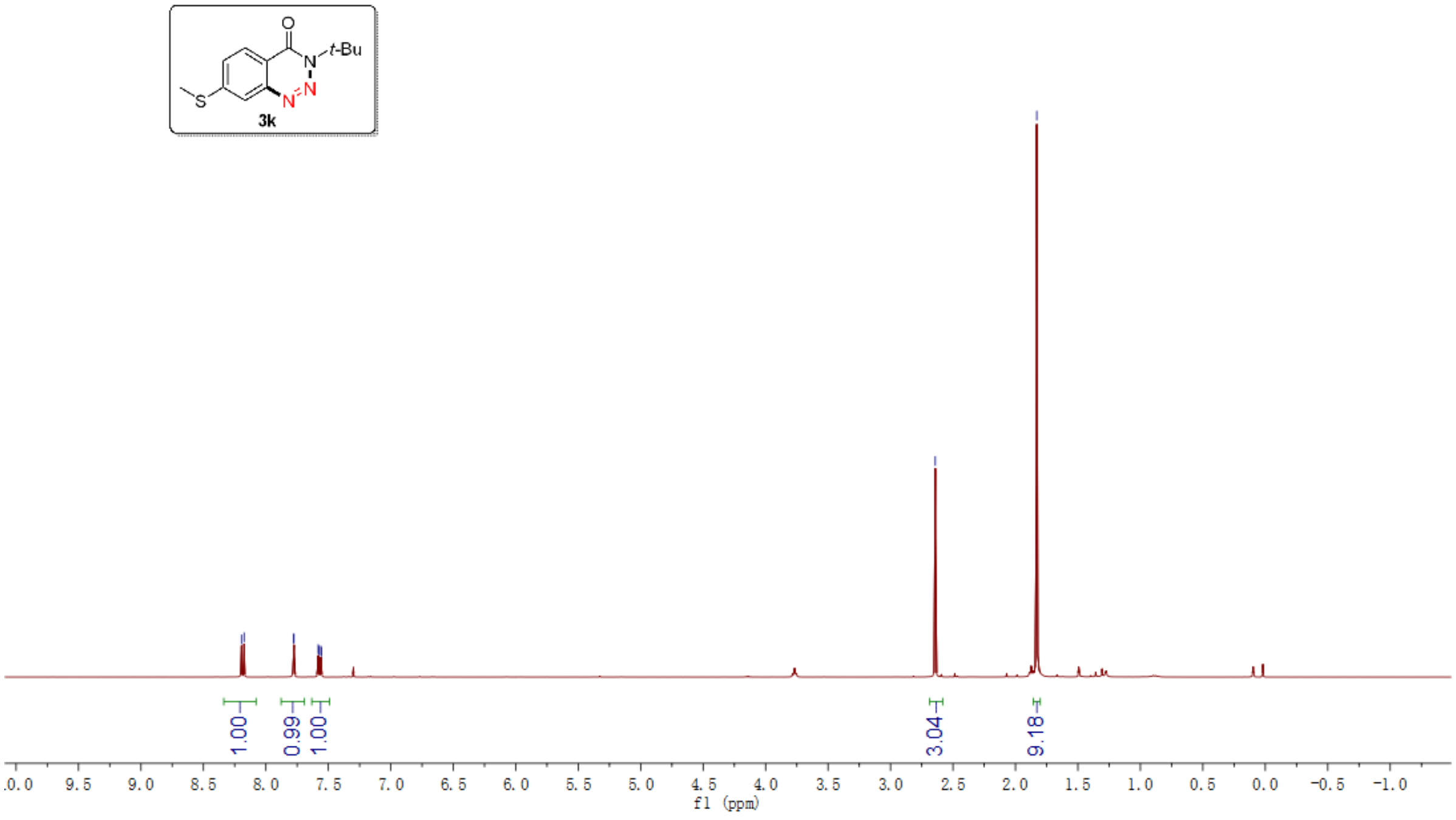
${ }^{13} \mathrm{C}$ - NMR spectrum of compound $\mathbf{3 k}\left(100 \mathrm{MHz}, \mathrm{CDCl}_{3}\right)$

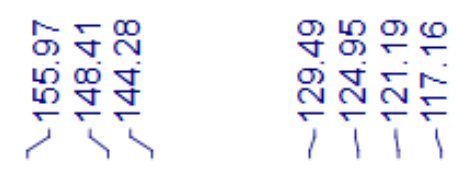

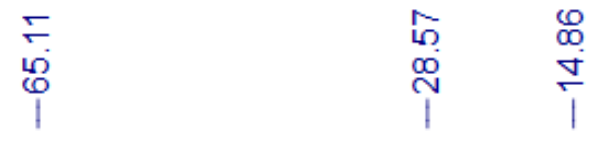
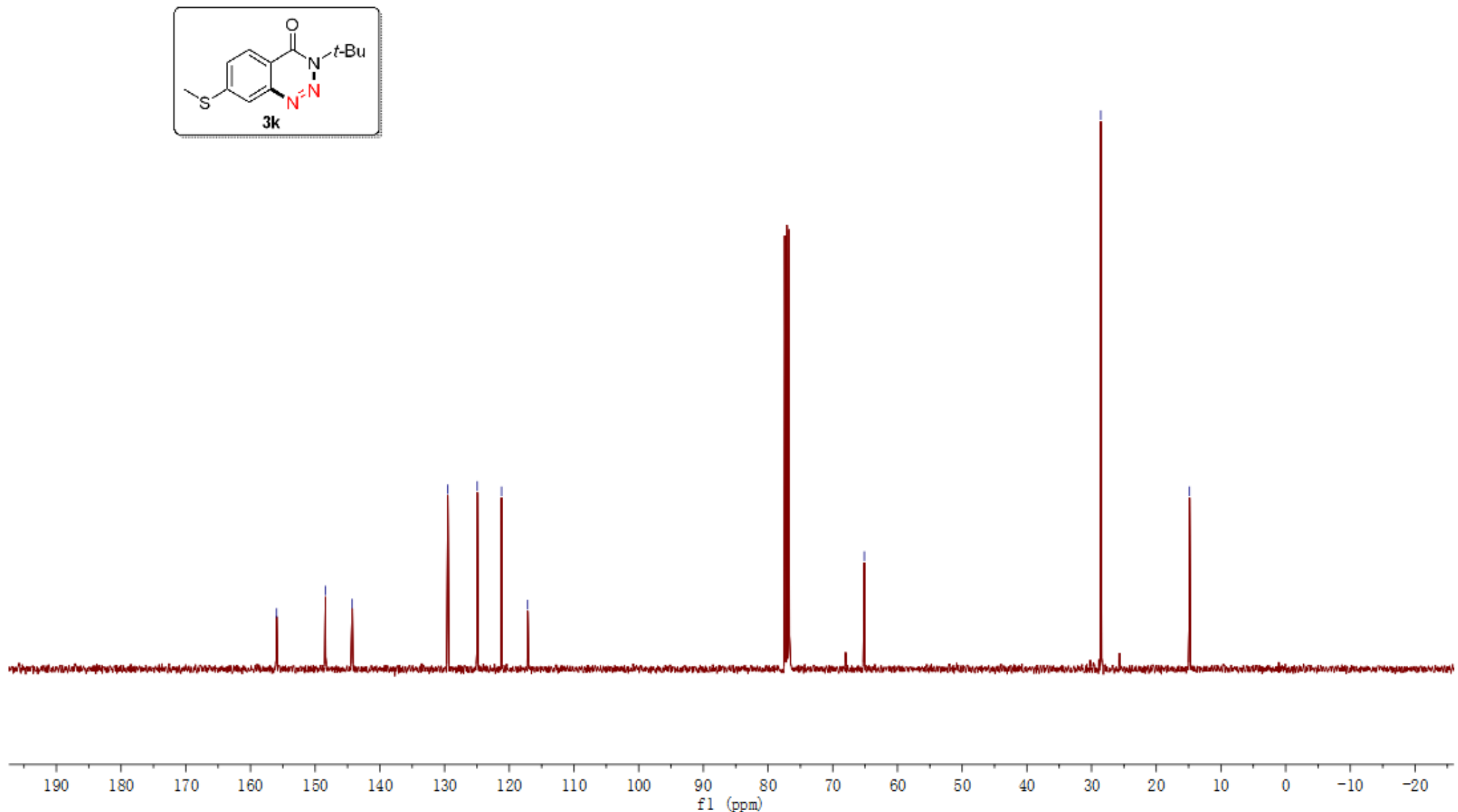
${ }^{1} \mathrm{H}$ - NMR spectrum of compound $3 \mathrm{I}\left(600 \mathrm{MHz}, \mathrm{CDCl}_{3}\right)$

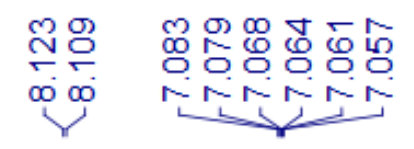

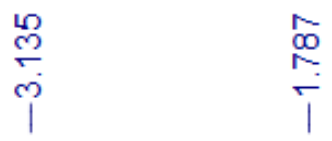
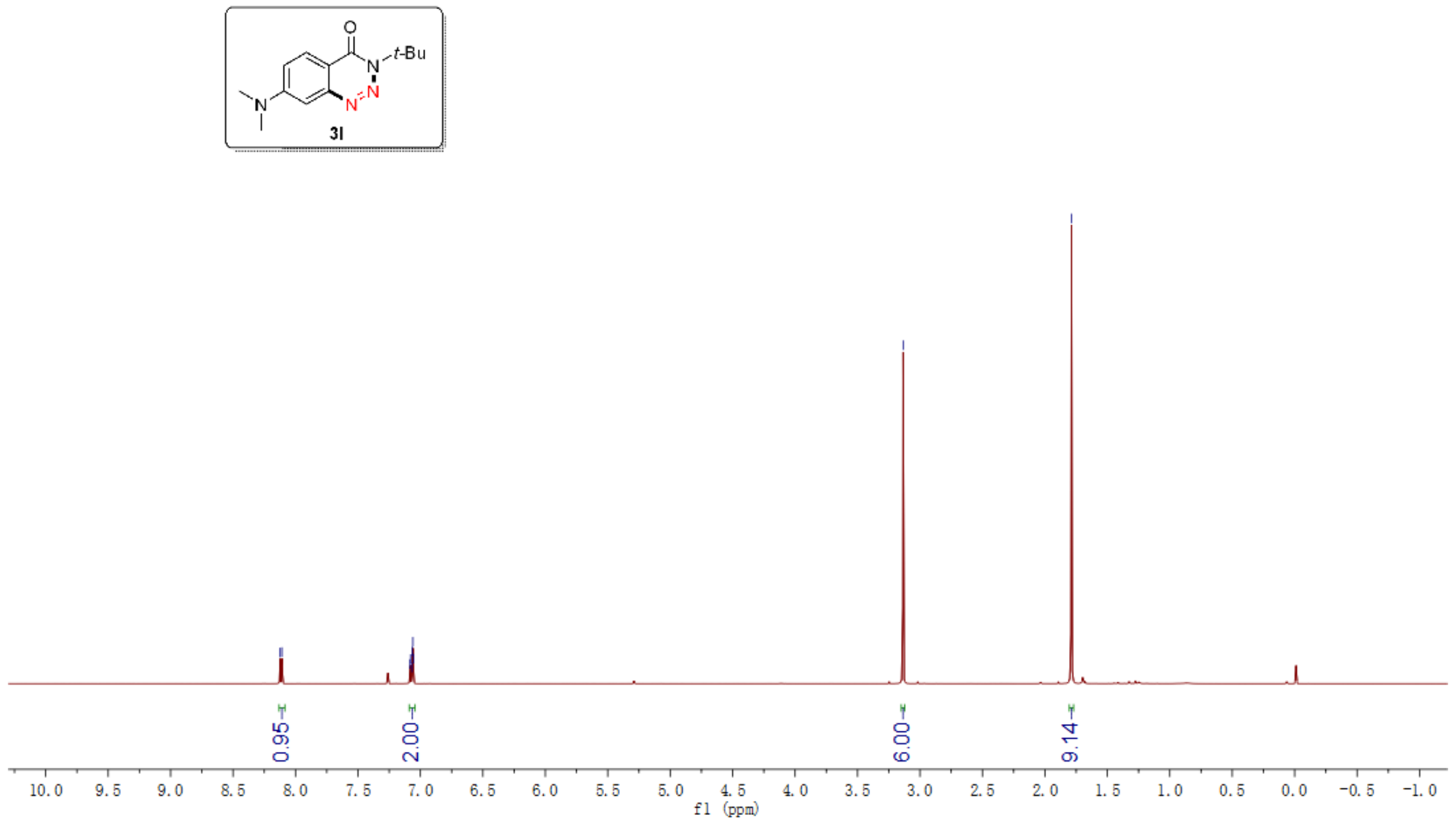
${ }^{13} \mathrm{C}$ - NMR spectrum of compound $31\left(100 \mathrm{MHz}, \mathrm{CDCl}_{3}\right)$

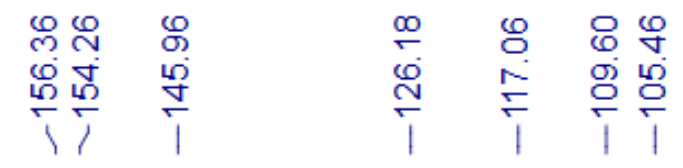

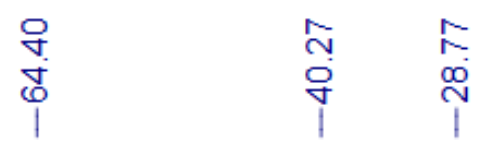
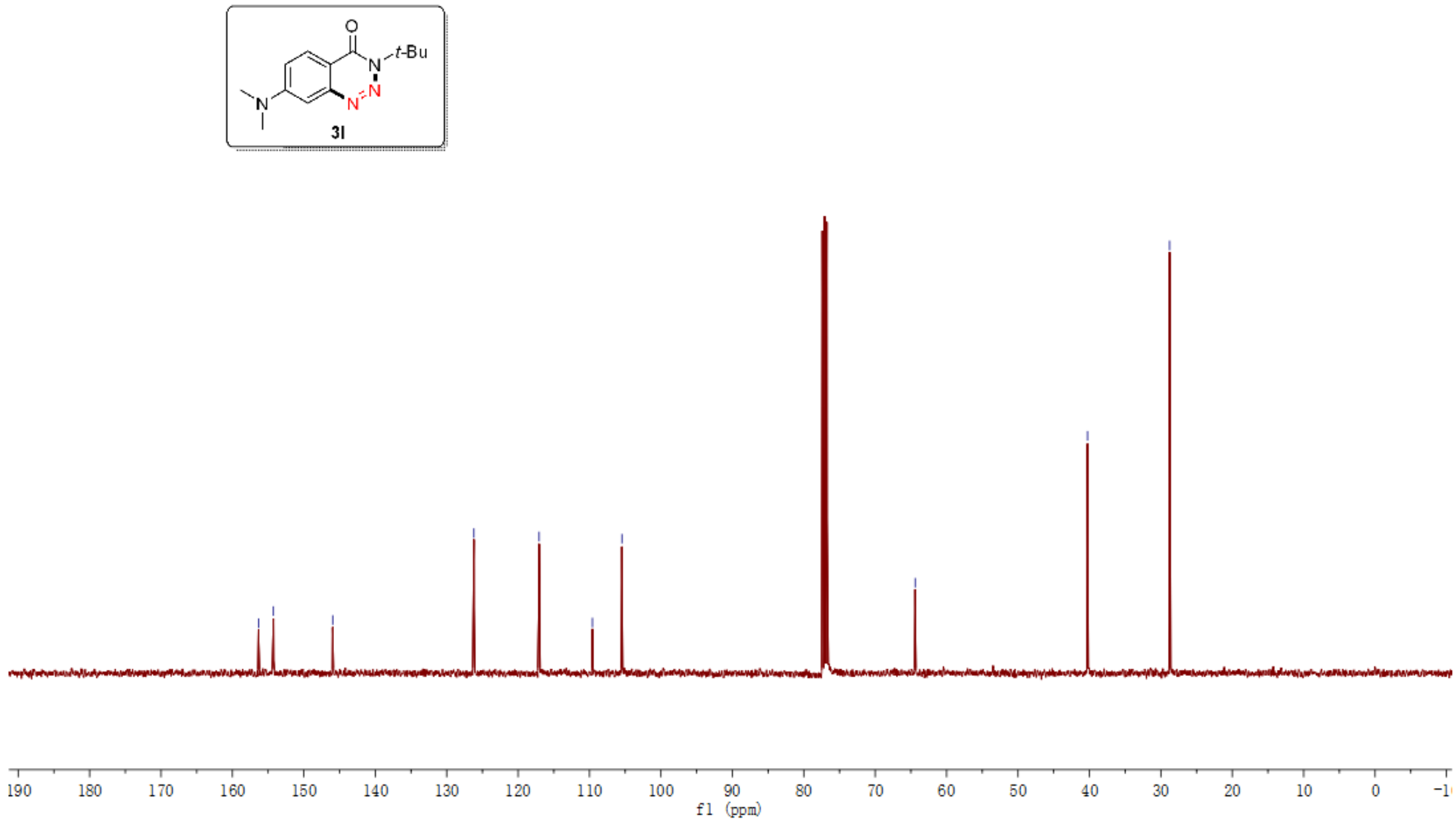
${ }^{1} \mathrm{H}$ - NMR spectrum of compound $\mathbf{3 m}\left(400 \mathrm{MHz}, \mathrm{CDCl}_{3}\right)$

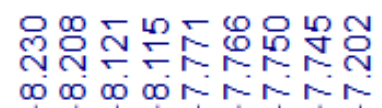

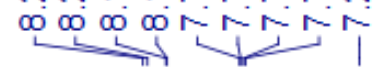

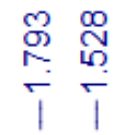

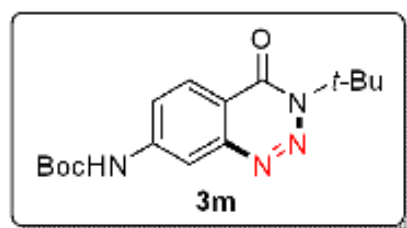

$3 \mathrm{~m}$

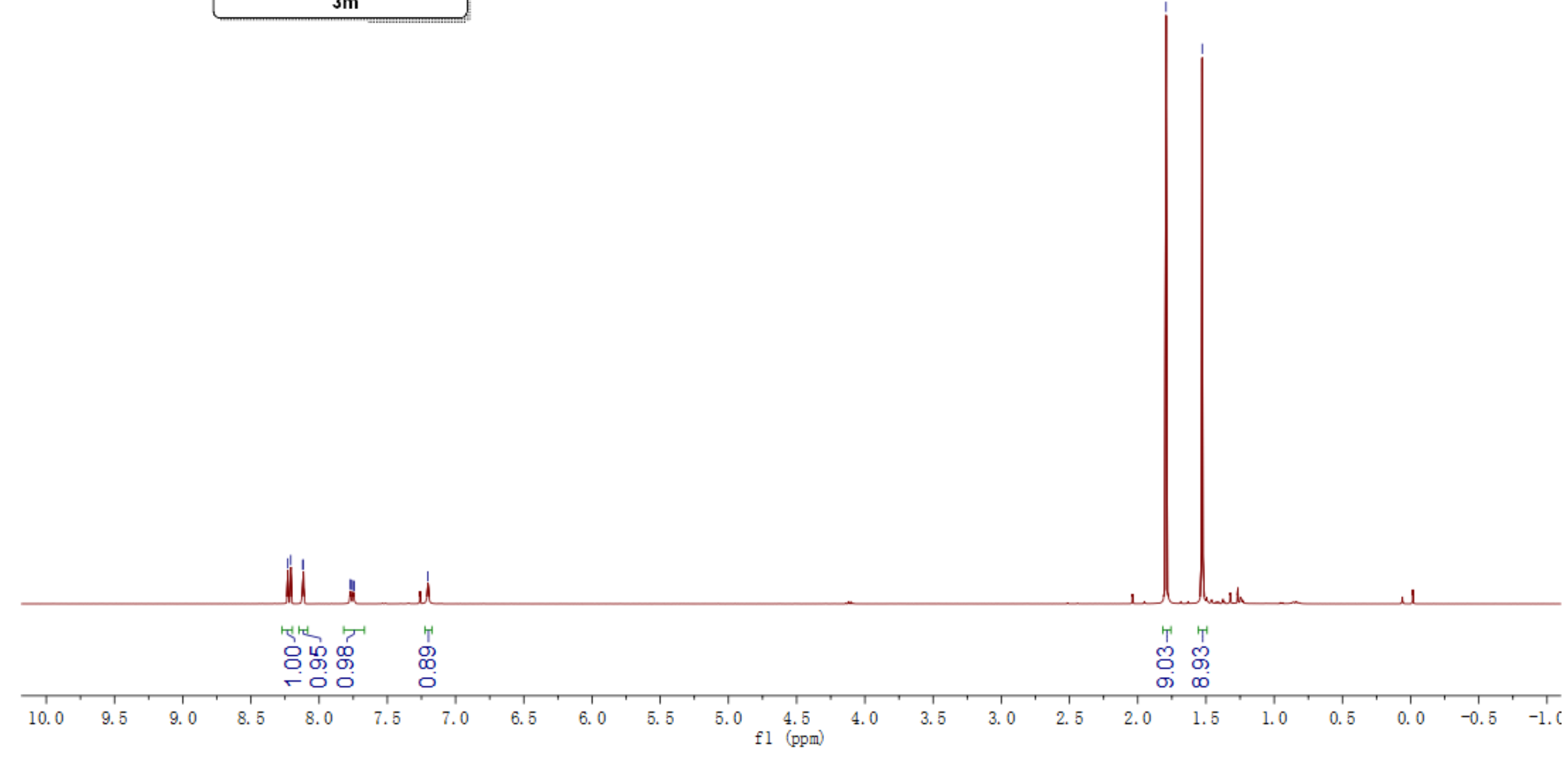


${ }^{13} \mathrm{C}$ - NMR spectrum of compound $\mathbf{3 m}\left(100 \mathrm{MHz}, \mathrm{CDCl}_{3}\right)$
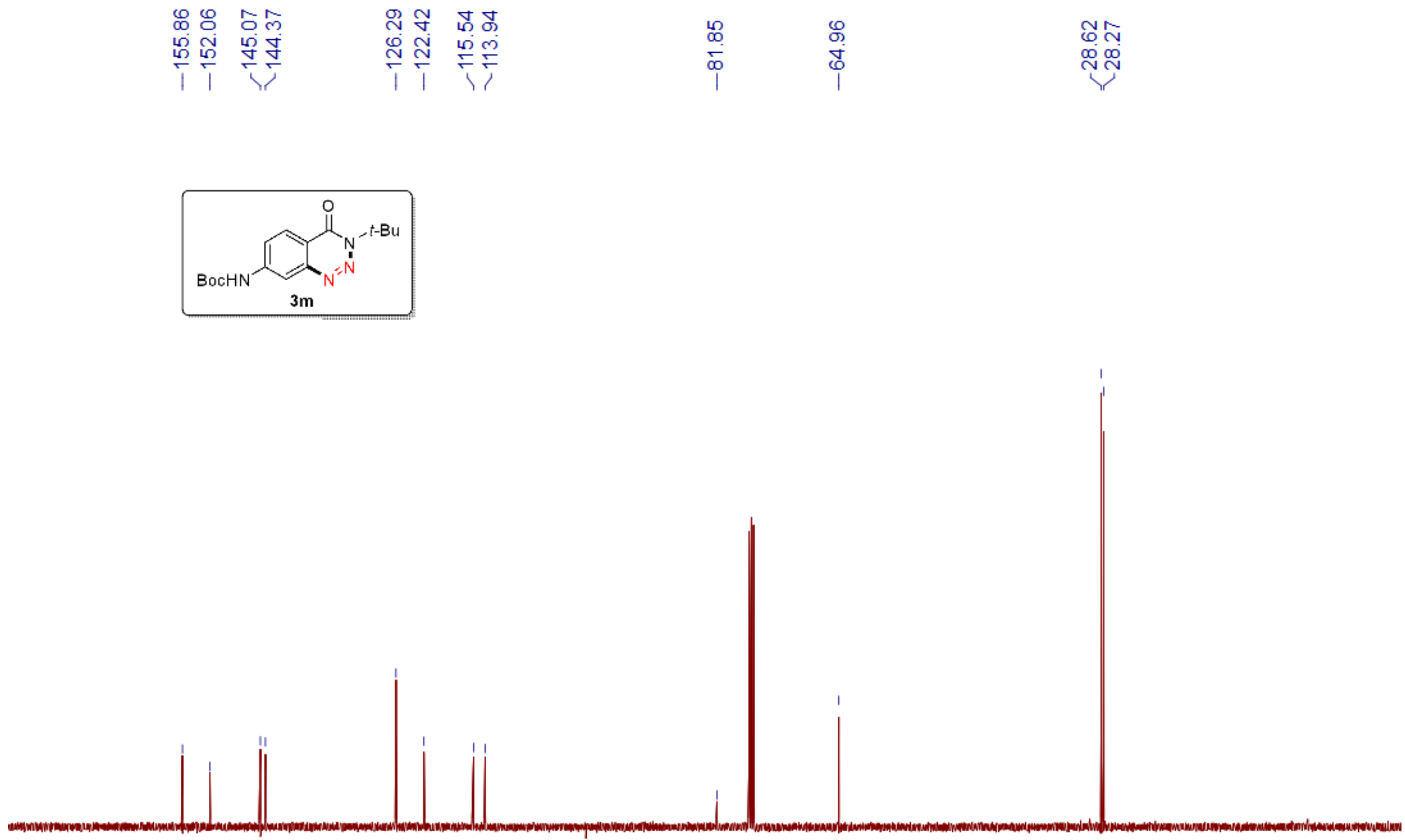
${ }^{1} \mathrm{H}$ - NMR spectrum of compound $\mathbf{3 n}\left(400 \mathrm{MHz}, \mathrm{CDCl}_{3}\right)$
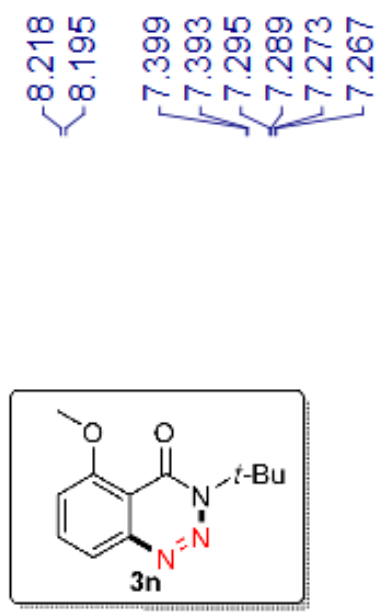

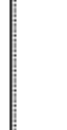

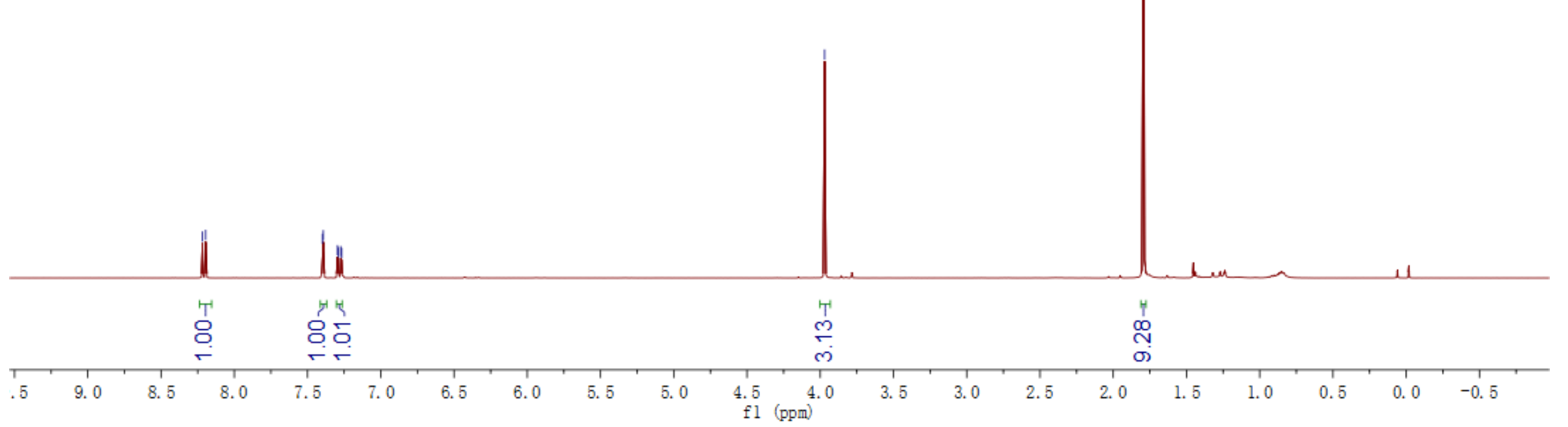


${ }^{13} \mathrm{C}$ - NMR spectrum of compound $3 \mathbf{n}\left(100 \mathrm{MHz}, \mathrm{CDCl}_{3}\right)$

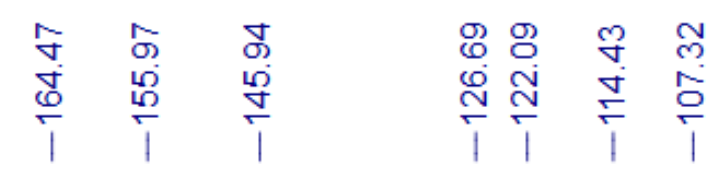

$\begin{array}{ll}0 & 8 \\ 0 & 0 \\ 0 & 0 \\ 1 & 1\end{array}$

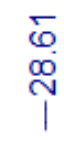
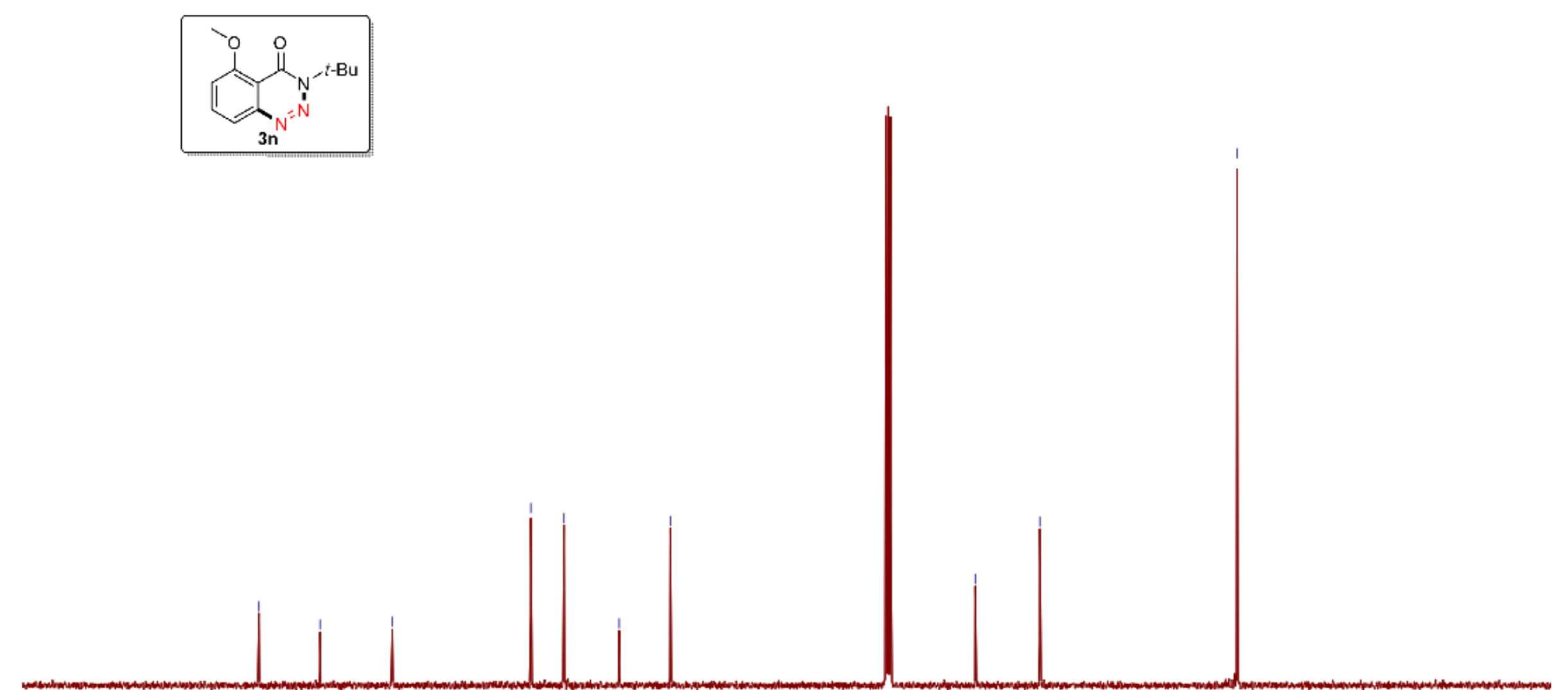
${ }^{1} \mathrm{H}$ - NMR spectrum of compound $\mathbf{3 o}\left(400 \mathrm{MHz}, \mathrm{CDCl}_{3}\right)$

ॠ

कु

$\stackrel{\infty}{\infty}$

$\stackrel{1}{\leftarrow}$
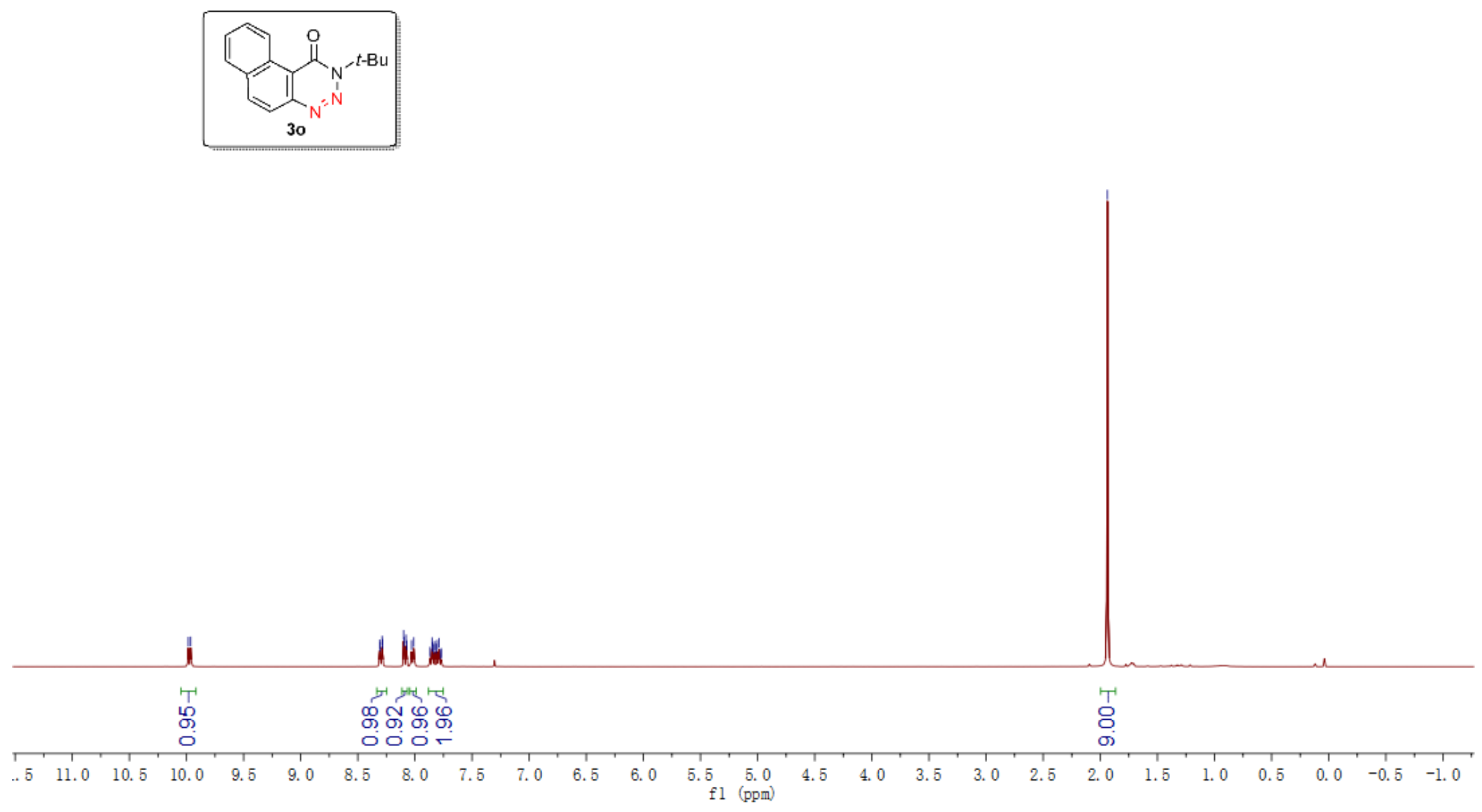
${ }^{13} \mathrm{C}$ - NMR spectrum of compound $3 \mathbf{3 o}\left(100 \mathrm{MHz}, \mathrm{CDCl}_{3}\right)$
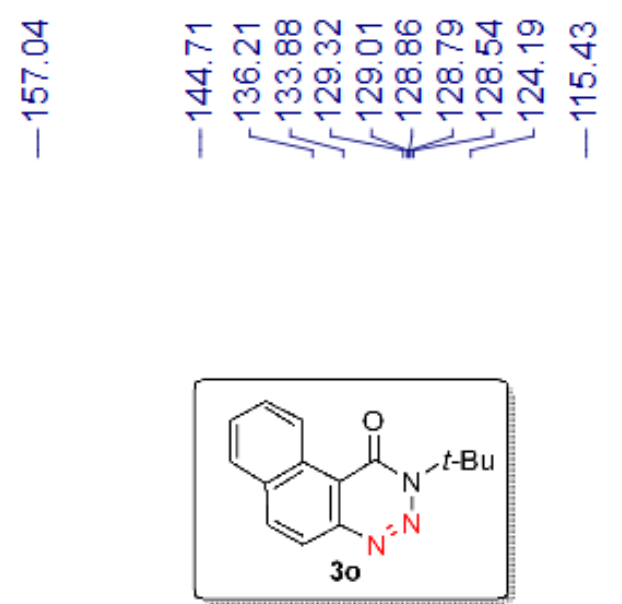

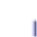

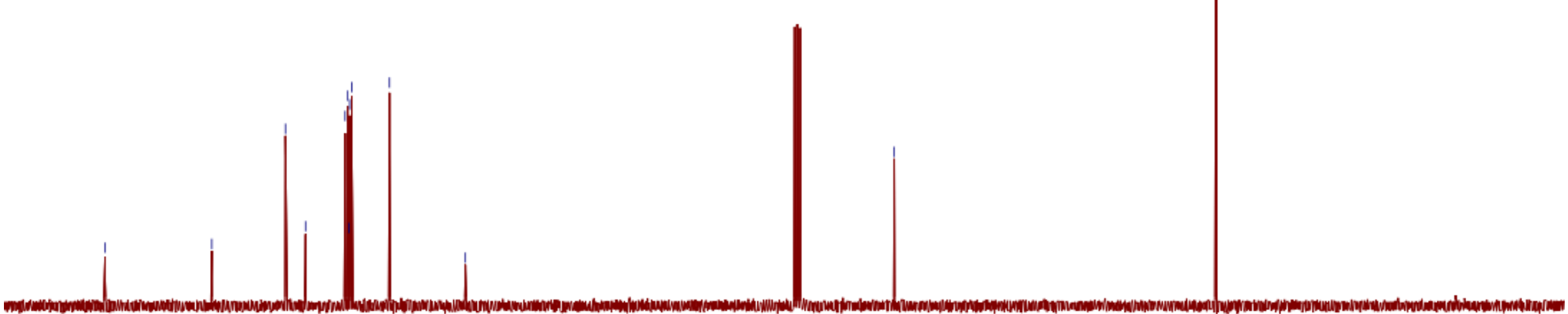

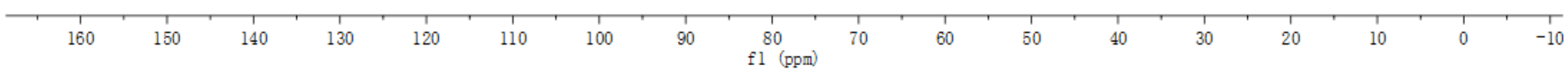


${ }^{1} \mathrm{H}$ - NMR spectrum of compound $\mathbf{3 p}\left(400 \mathrm{MHz}, \mathrm{CDCl}_{3}\right)$

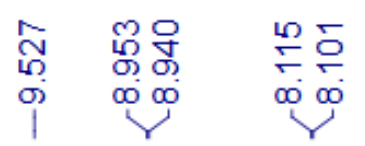
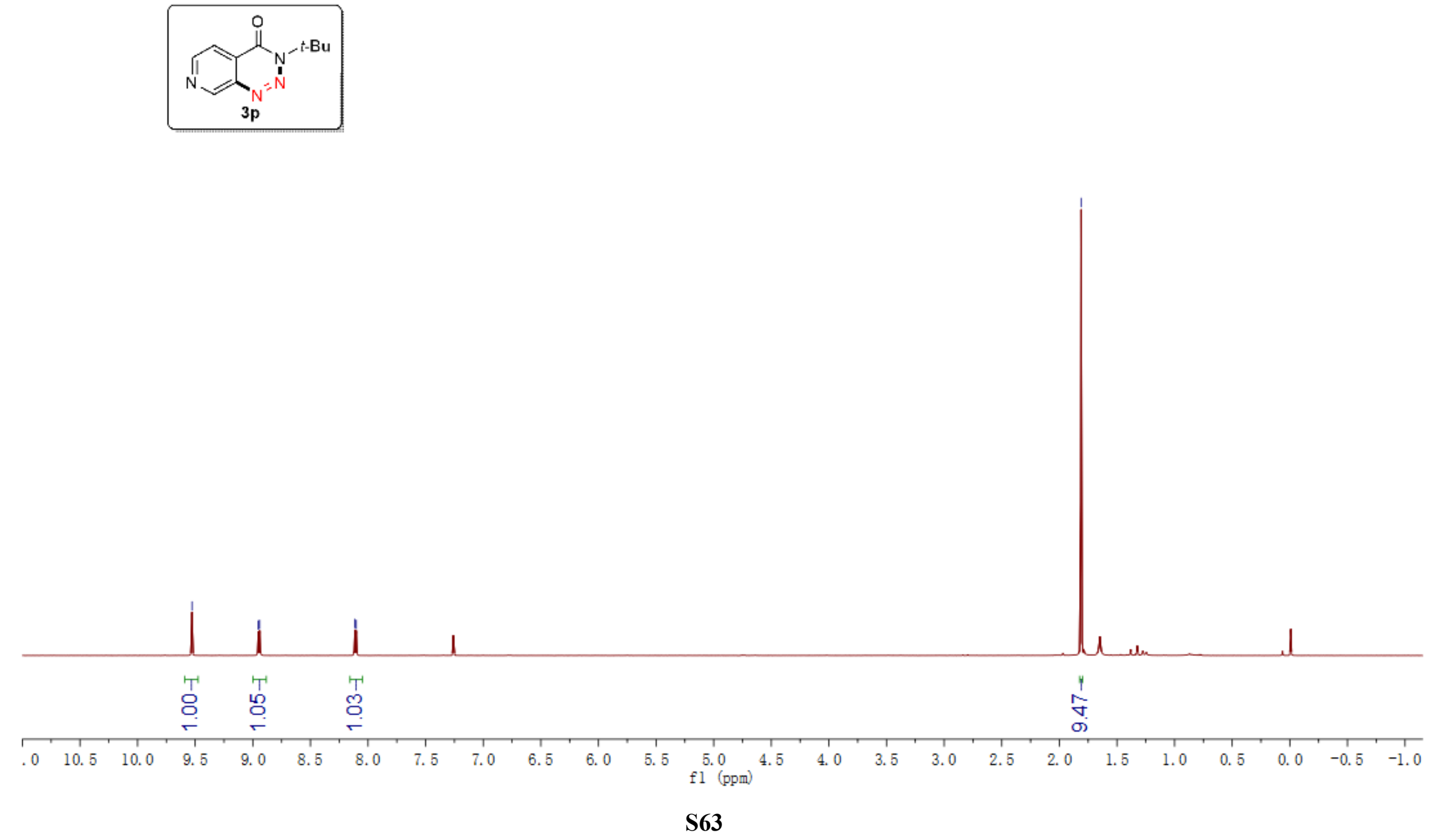
${ }^{13} \mathrm{C}$ - NMR spectrum of compound $\mathbf{3 p}\left(100 \mathrm{MHz}, \mathrm{CDCl}_{3}\right)$

$$
\text { 舟两 }
$$
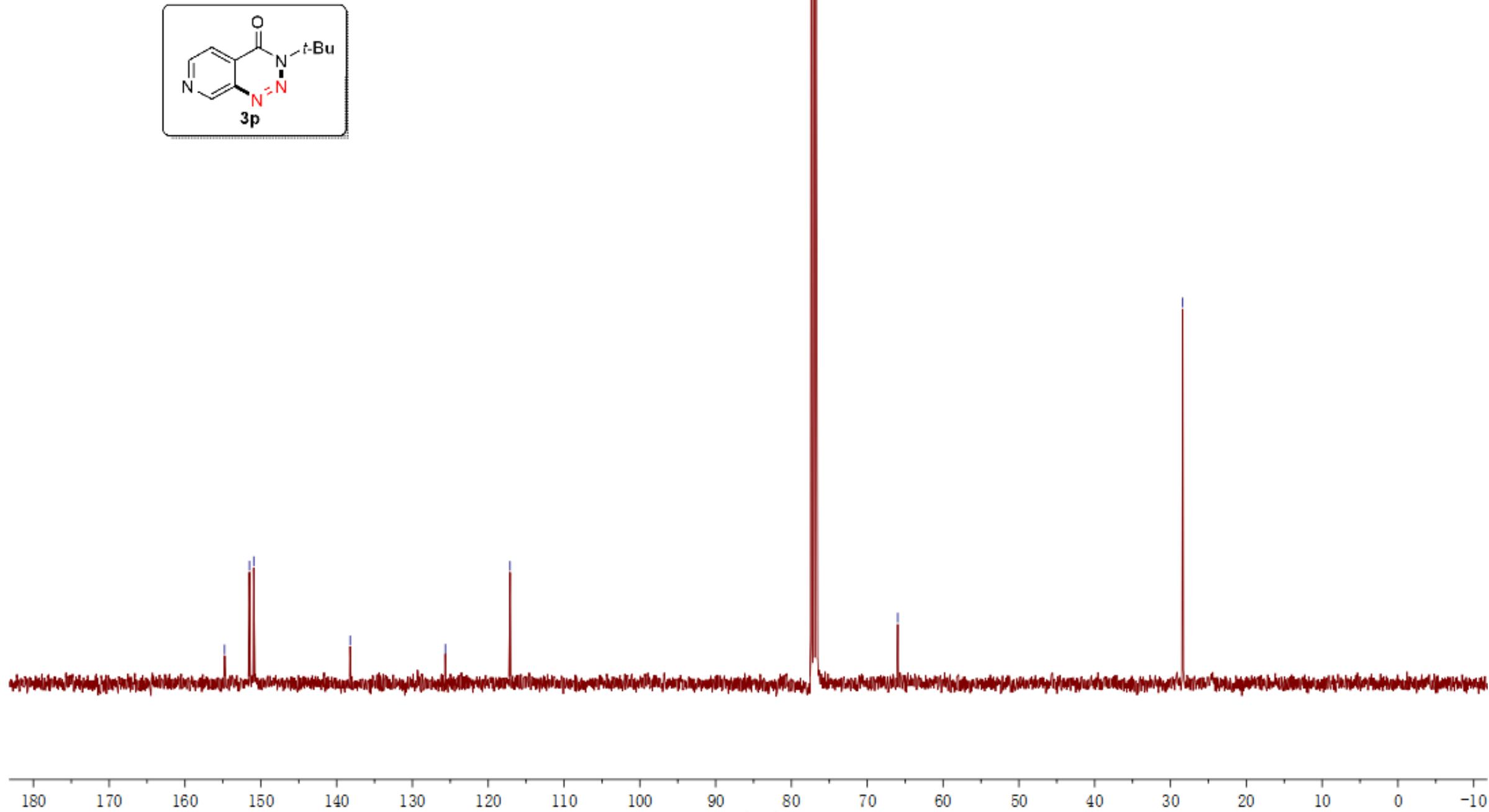

$140 \quad 130$

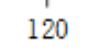

110 
${ }^{1} \mathrm{H}$ - NMR spectrum of compound $\mathbf{3 q}\left(400 \mathrm{MHz}, \mathrm{CDCl}_{3}\right)$
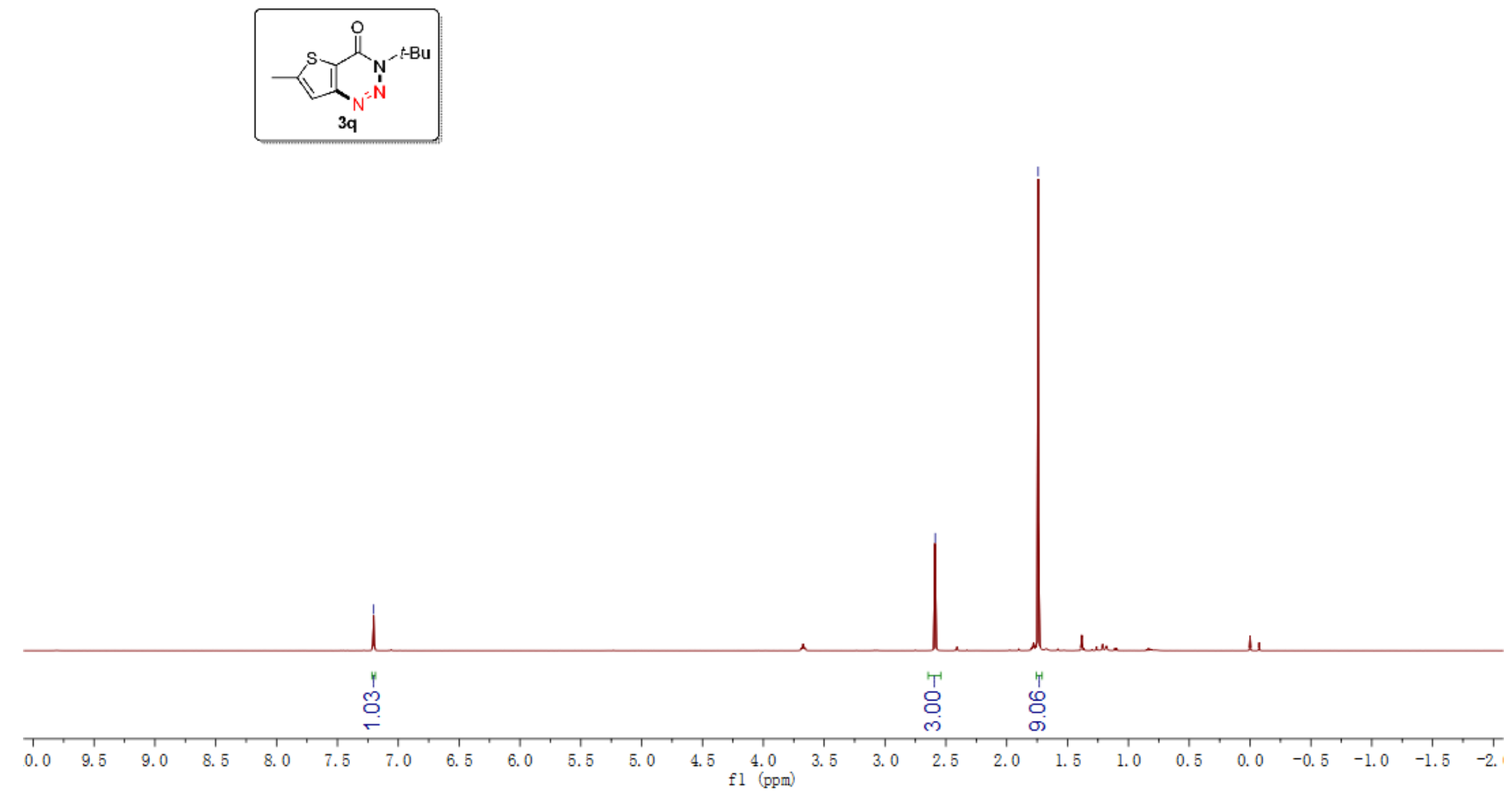
${ }^{13} \mathrm{C}$ - NMR spectrum of compound $\mathbf{3 q}\left(100 \mathrm{MHz}, \mathrm{CDCl}_{3}\right)$

落

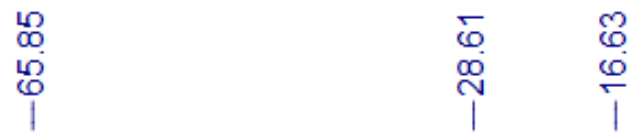
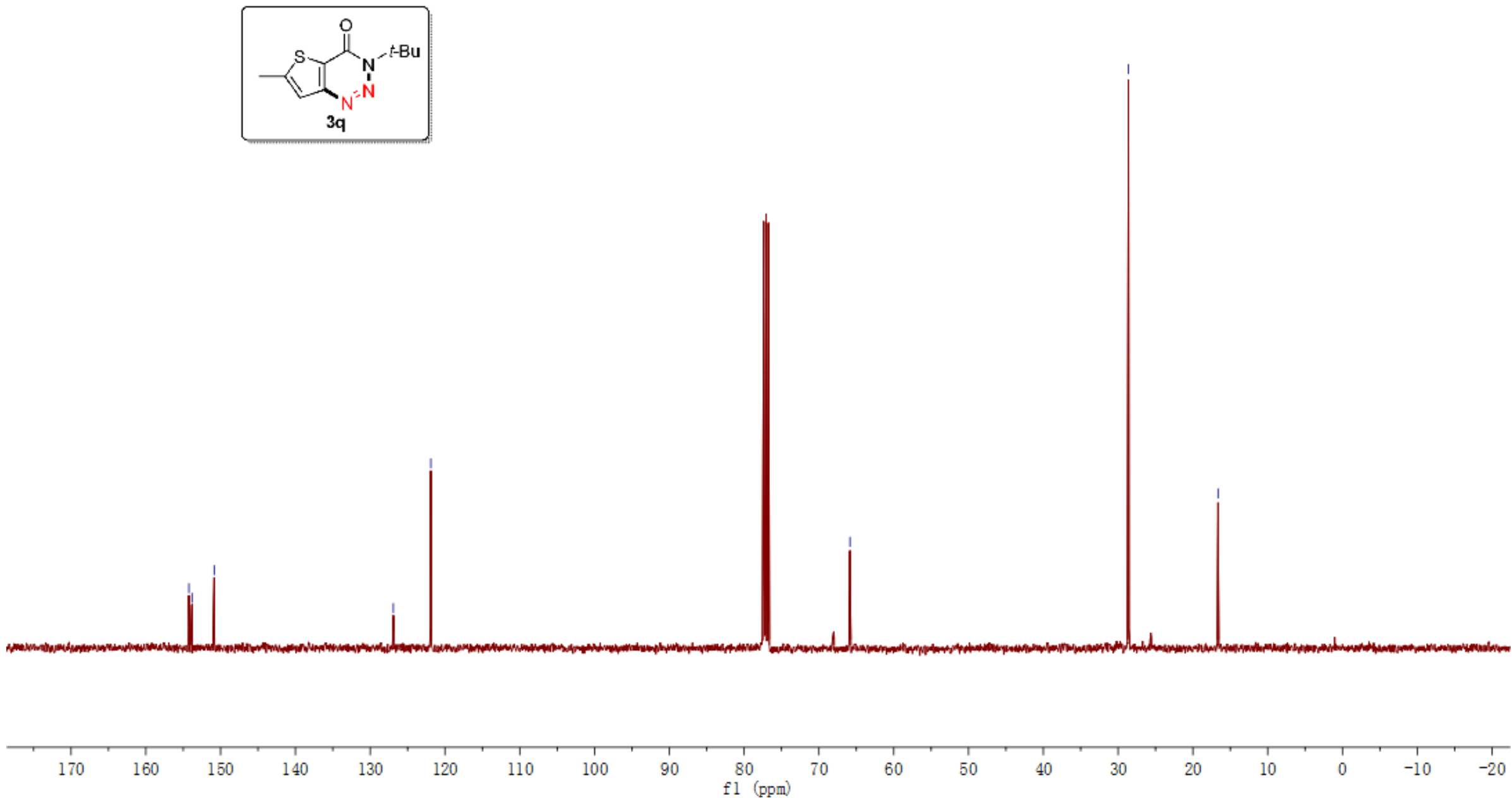
${ }^{1} \mathrm{H}$ - NMR spectrum of compound $\mathbf{3 r}\left(600 \mathrm{MHz}, \mathrm{CDCl}_{3}\right)$

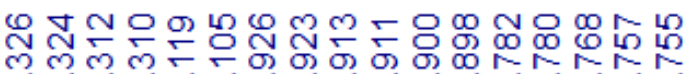
$\infty \infty \infty \infty \infty \infty \cdots-N-\infty$
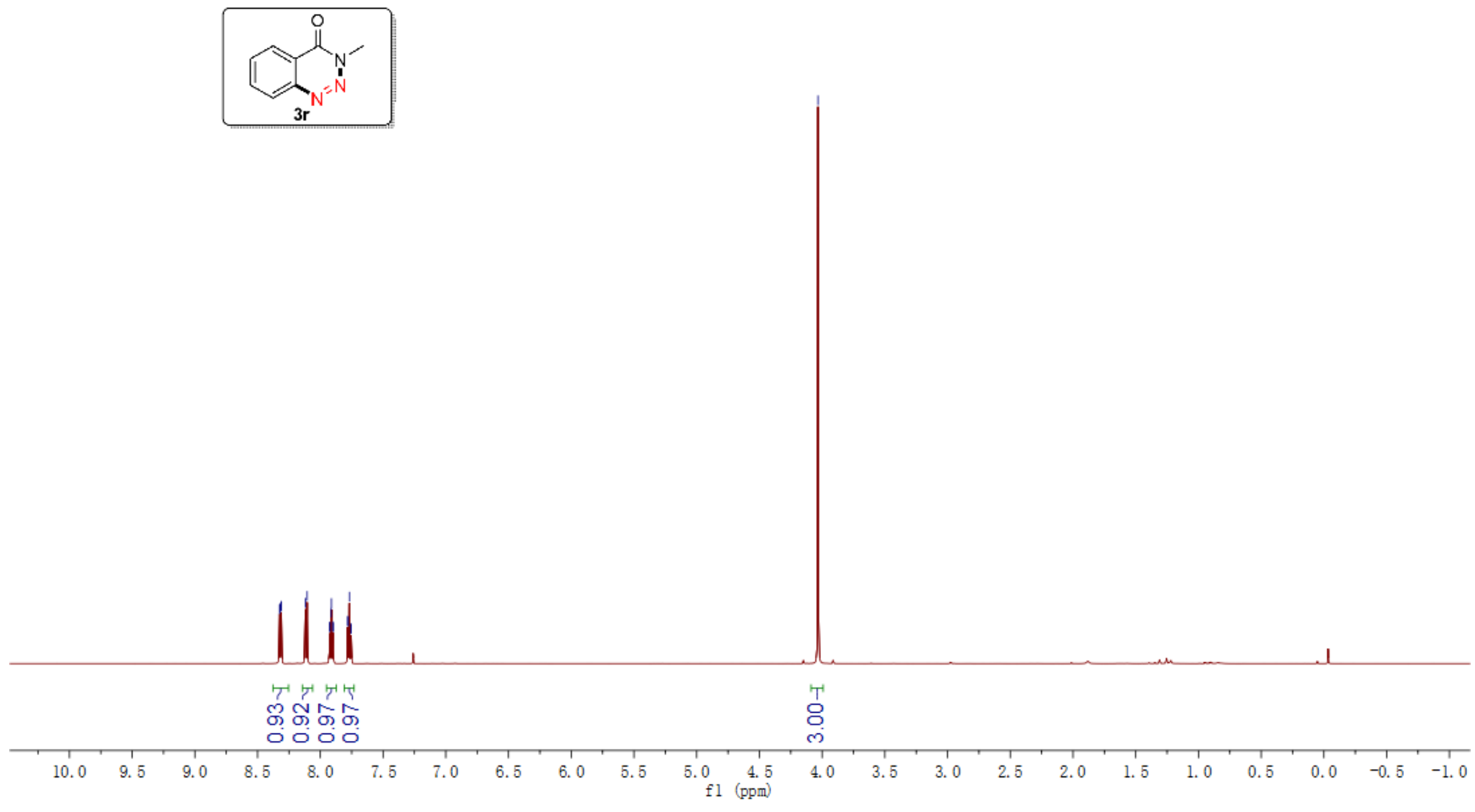
${ }^{13} \mathrm{C}$ - NMR spectrum of compound $\mathbf{3 r}\left(100 \mathrm{MHz}, \mathrm{CDCl}_{3}\right)$

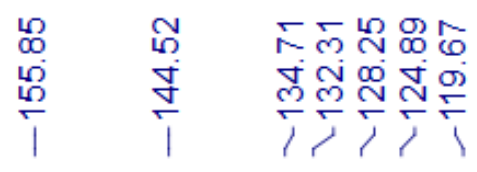

m

m
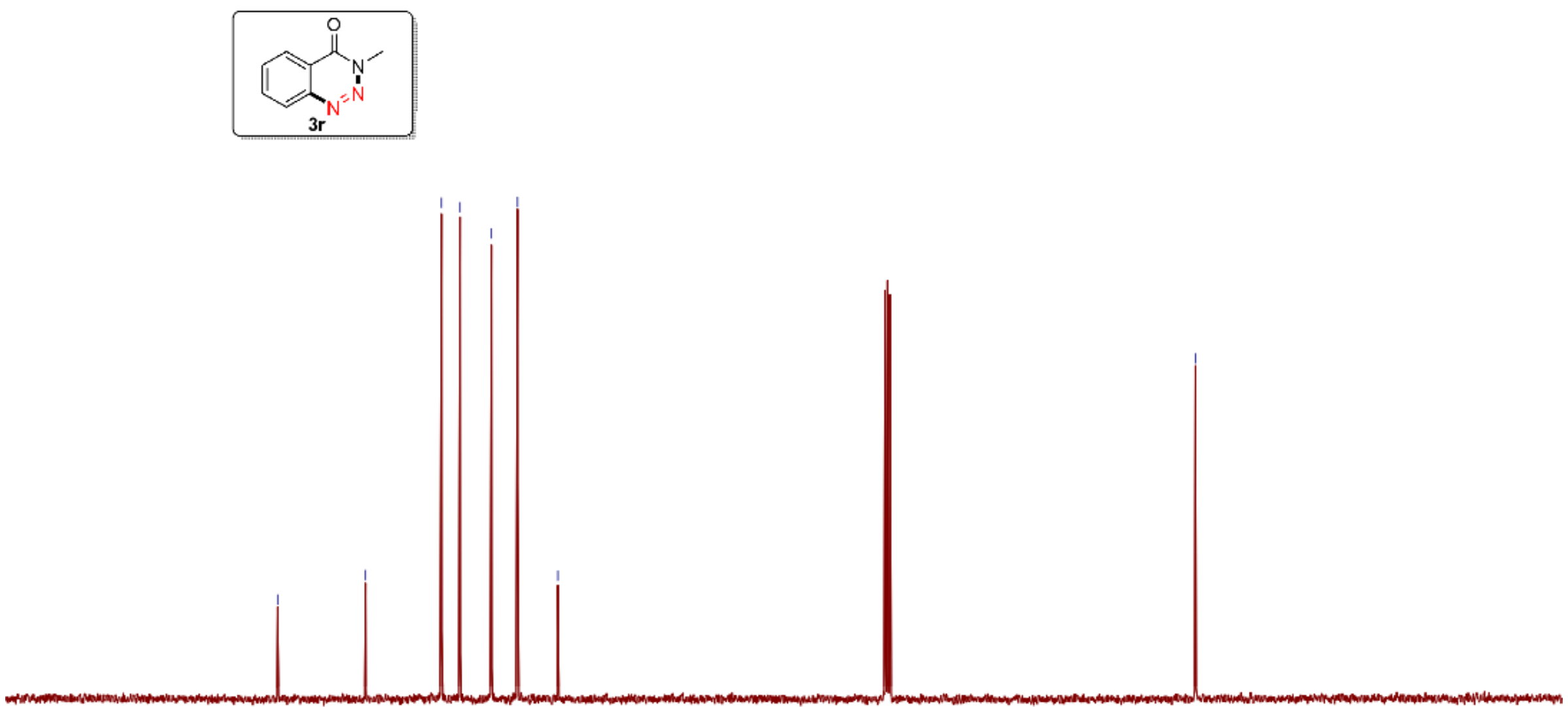

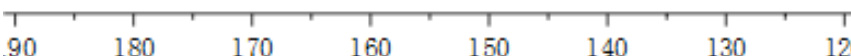

110

100

fl $\stackrel{90}{(\mathrm{ppm})}$

$\begin{array}{lll}1 & 1 \\ 80 & 70\end{array}$

60

50

40

30 
${ }^{1} \mathrm{H}$ - NMR spectrum of compound $3 \mathbf{s}\left(400 \mathrm{MHz}, \mathrm{CDCl}_{3}\right)$

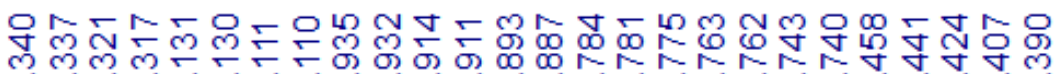

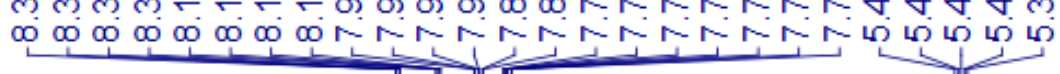
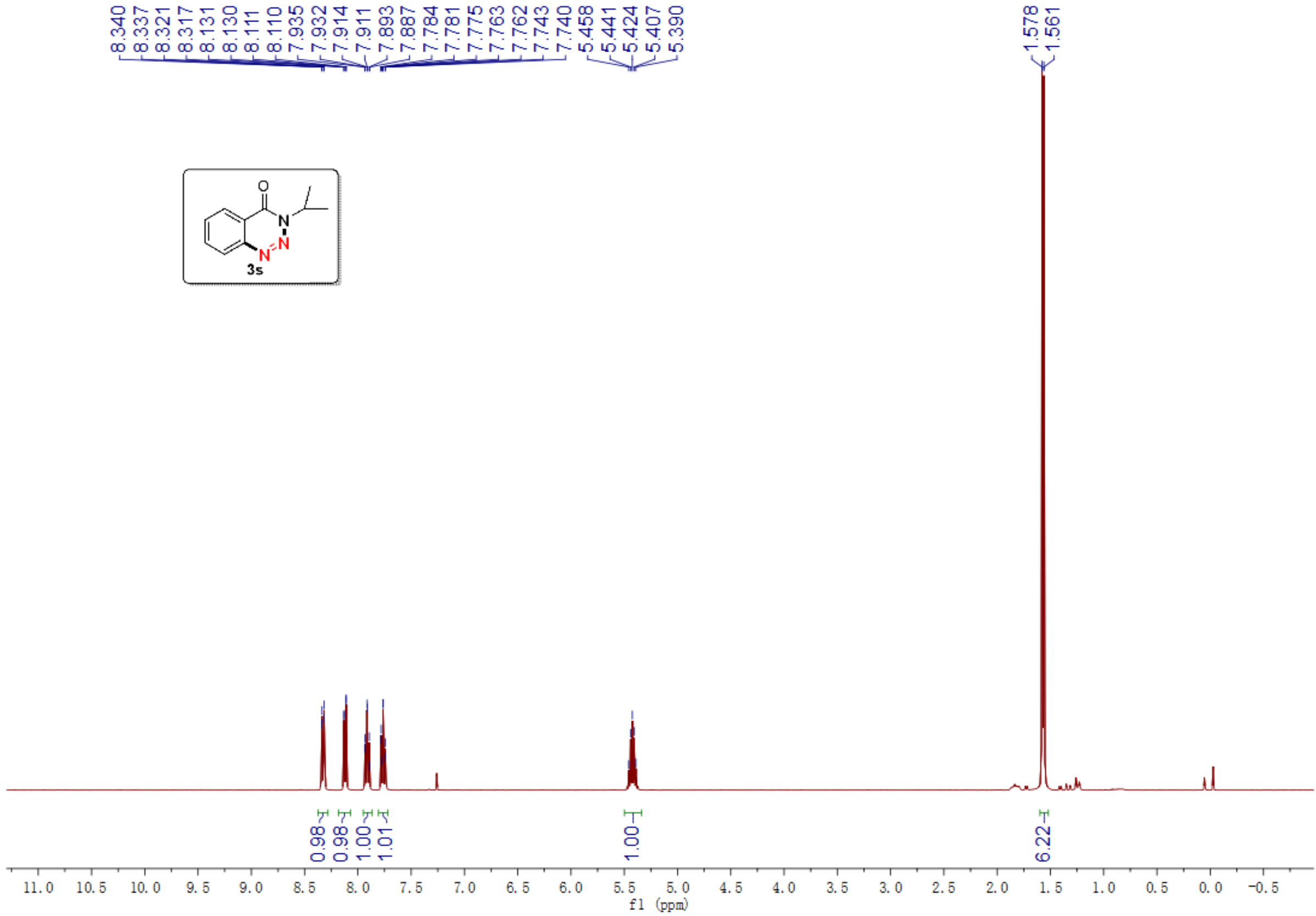
${ }^{13} \mathrm{C}$ - NMR spectrum of compound $3 \mathbf{s}\left(100 \mathrm{MHz}, \mathrm{CDCl}_{3}\right)$

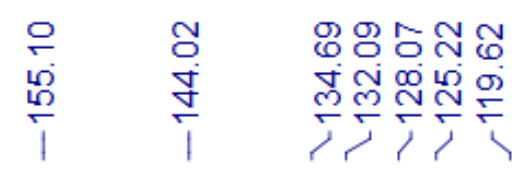

怘

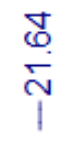
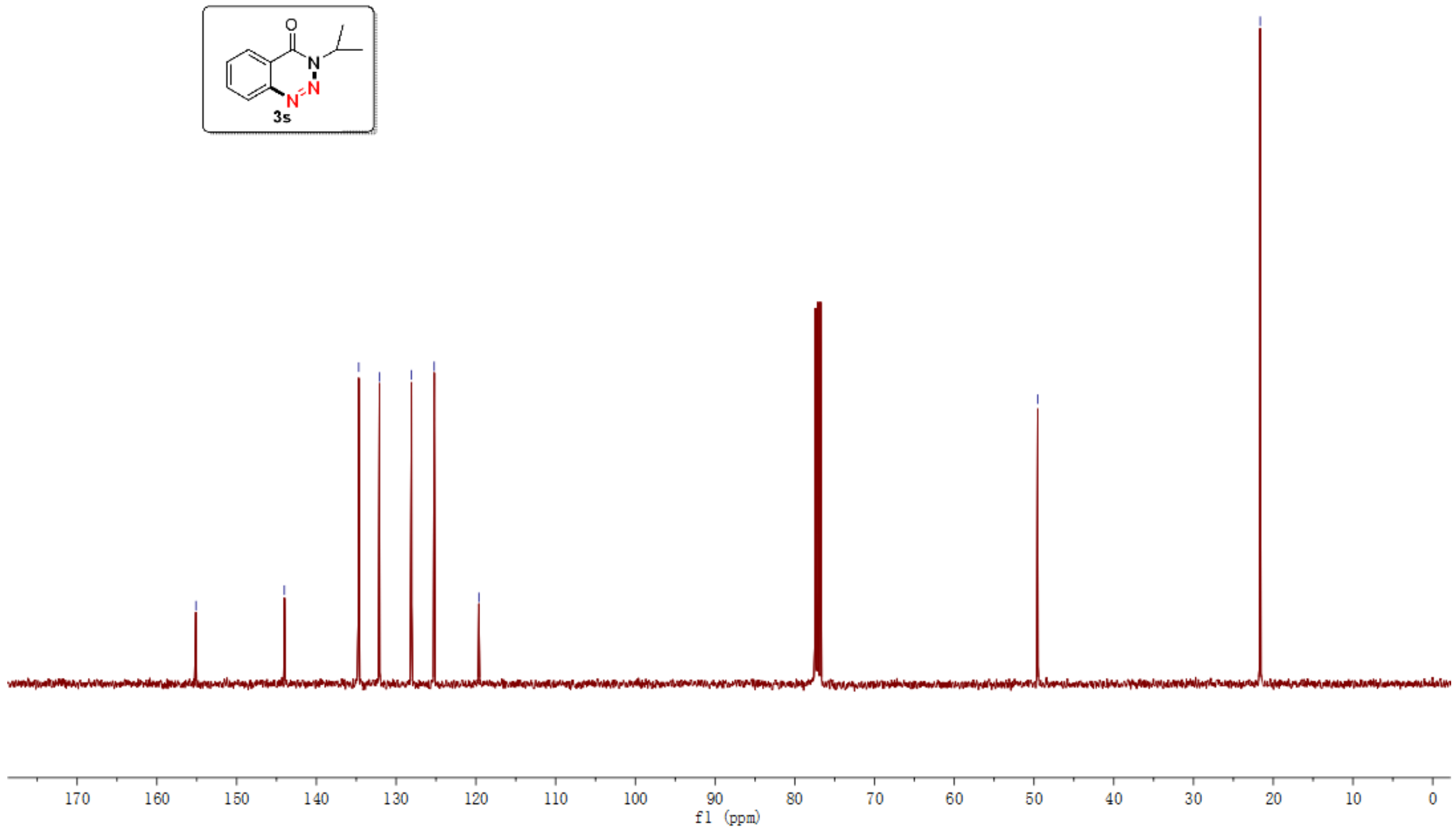
${ }^{1} \mathrm{H}$ - NMR spectrum of compound $\mathbf{3 t}\left(400 \mathrm{MHz}, \mathrm{CDCl}_{3}\right)$

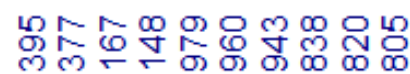

$\infty \infty \infty \infty \cdots \cdots$

กำ

讨

ํํำ

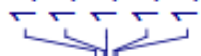
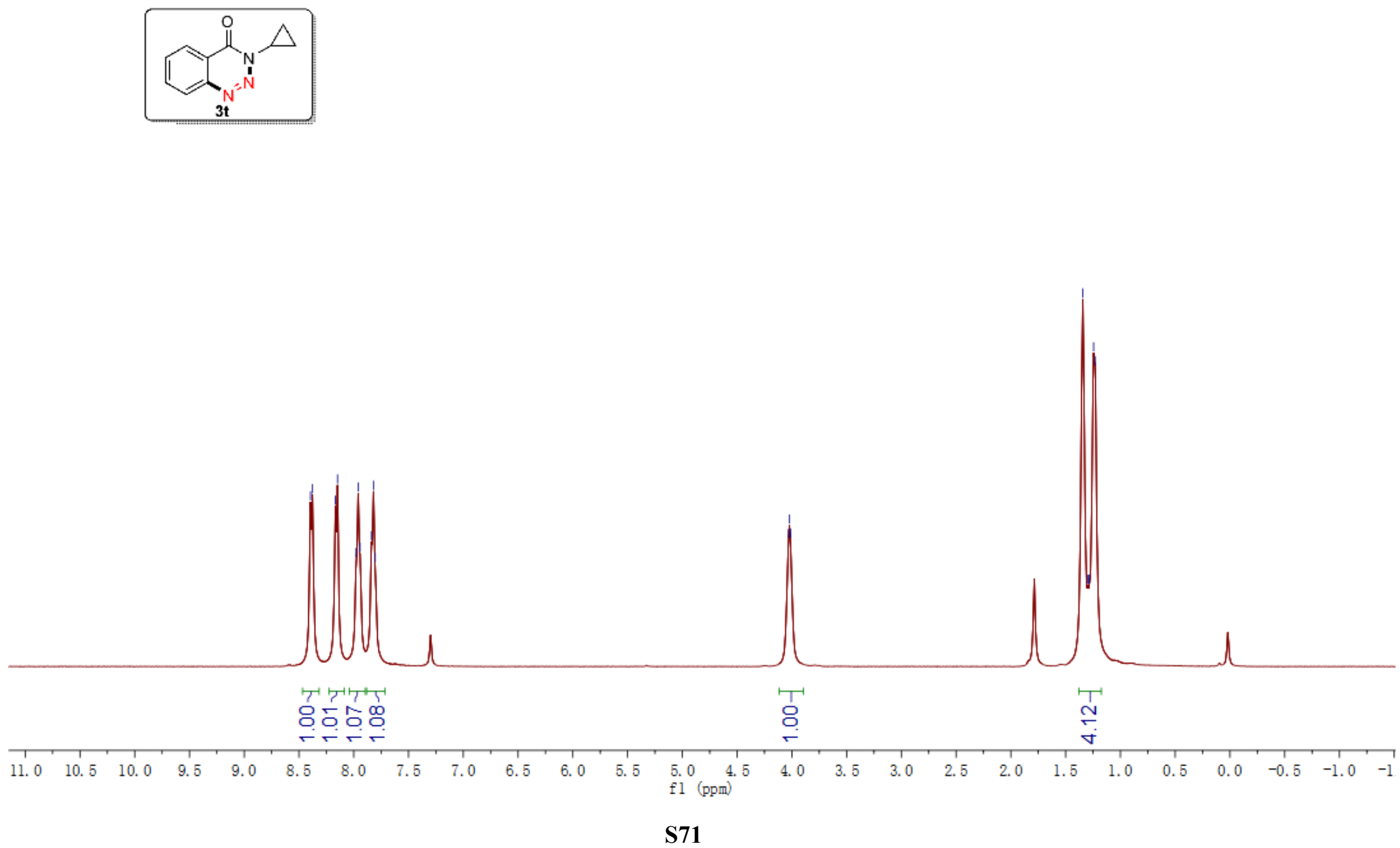
${ }^{13} \mathrm{C}$ - NMR spectrum of compound $\mathbf{3 t}\left(100 \mathrm{MHz}, \mathrm{CDCl}_{3}\right)$

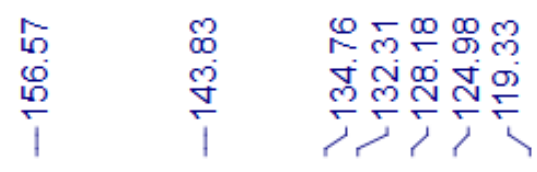
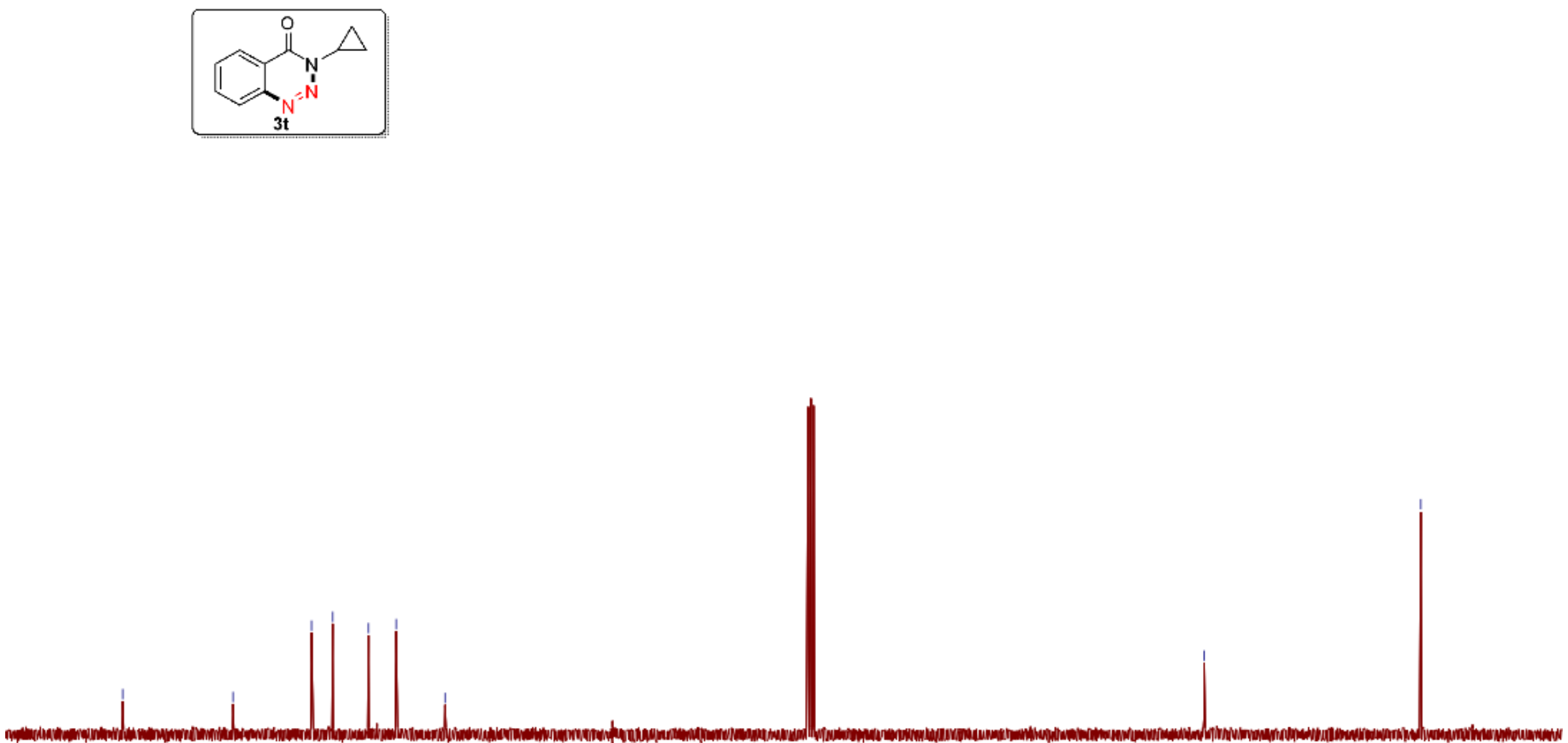

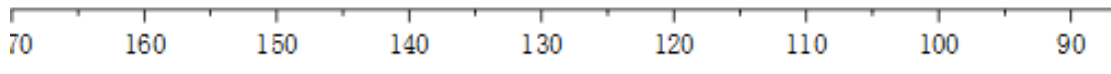


${ }^{1} \mathrm{H}$ - NMR spectrum of compound $\mathbf{3 u}\left(400 \mathrm{MHz}, \mathrm{CDCl}_{3}\right)$

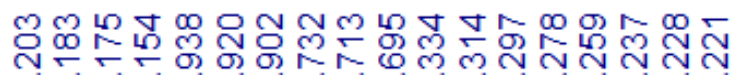

$\infty \infty \infty \infty \cdots$
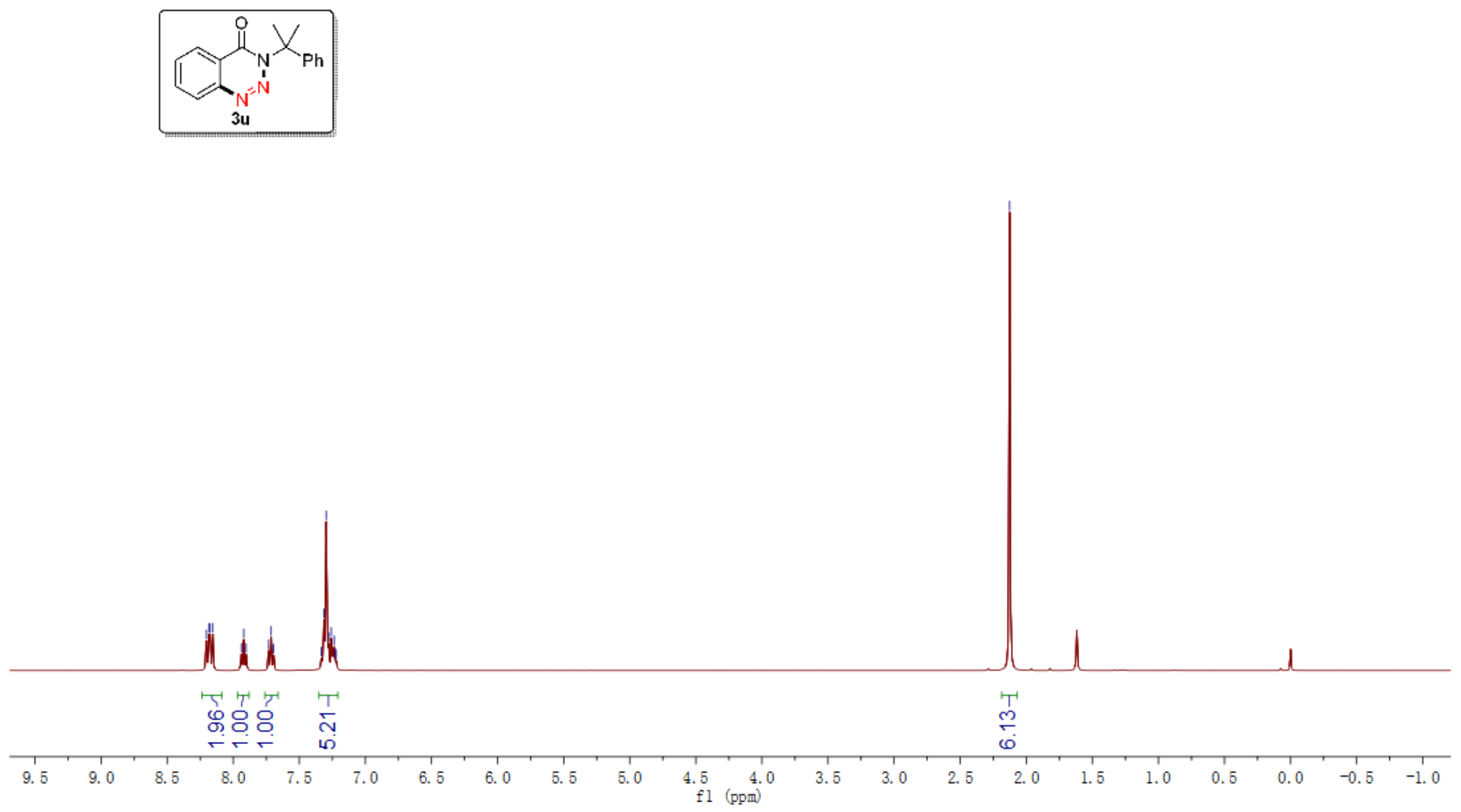
${ }^{13} \mathrm{C}$ - NMR spectrum of compound $\mathbf{3 u}\left(100 \mathrm{MHz}, \mathrm{CDCl}_{3}\right)$

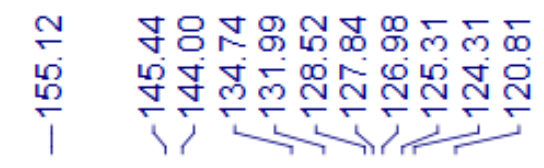

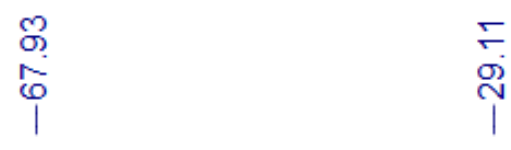
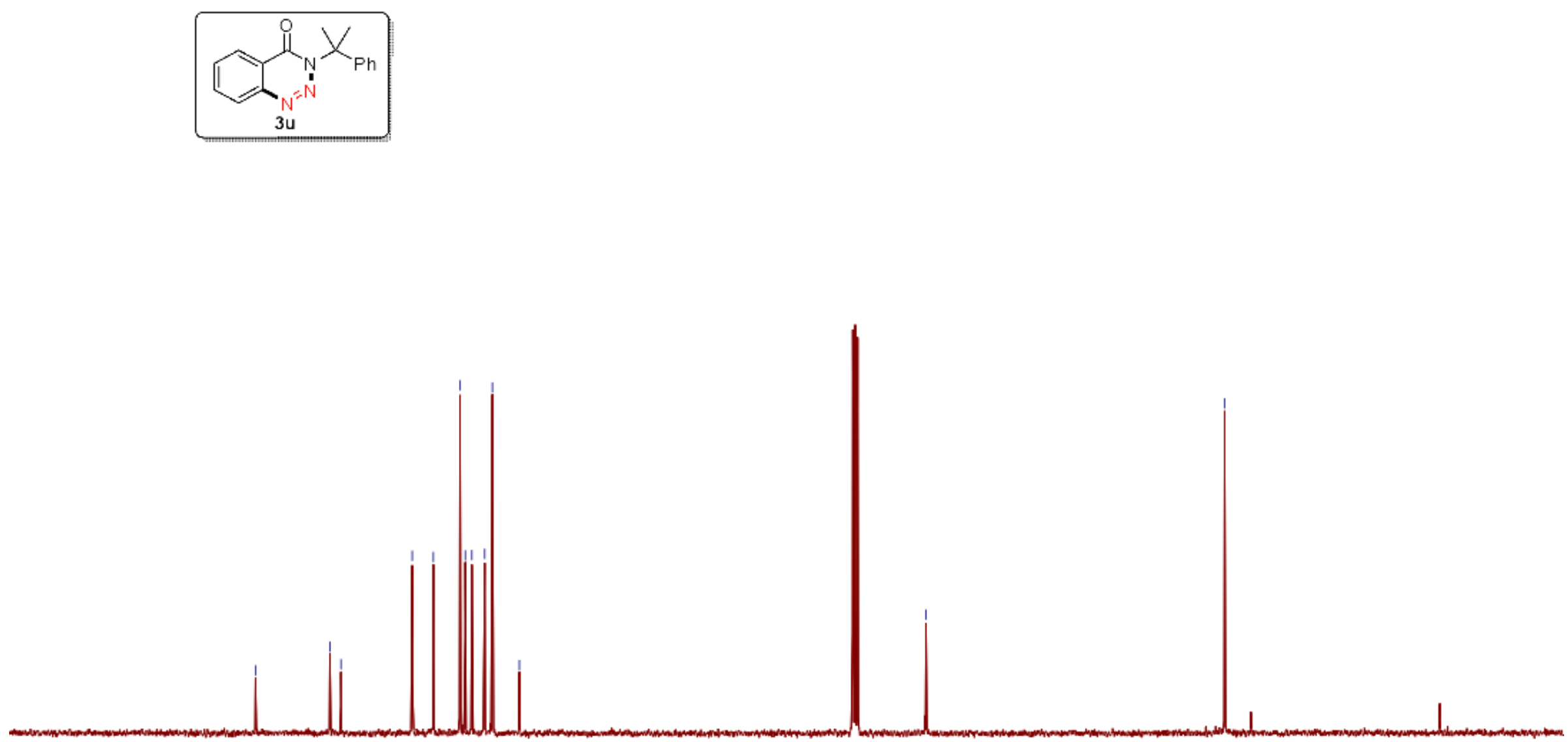

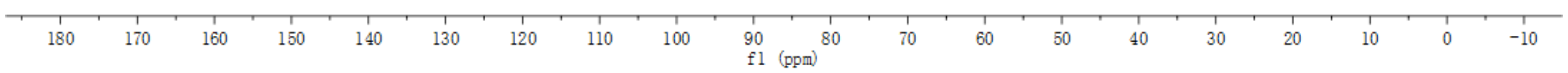


${ }^{1} \mathrm{H}$ - NMR spectrum of compound $\mathbf{3 v}\left(600 \mathrm{MHz}, \mathrm{CDCl}_{3}\right)$

ল্ল

$\infty \infty \infty \infty \infty \infty \cdots+\cdots$

พั่ํํำ

in
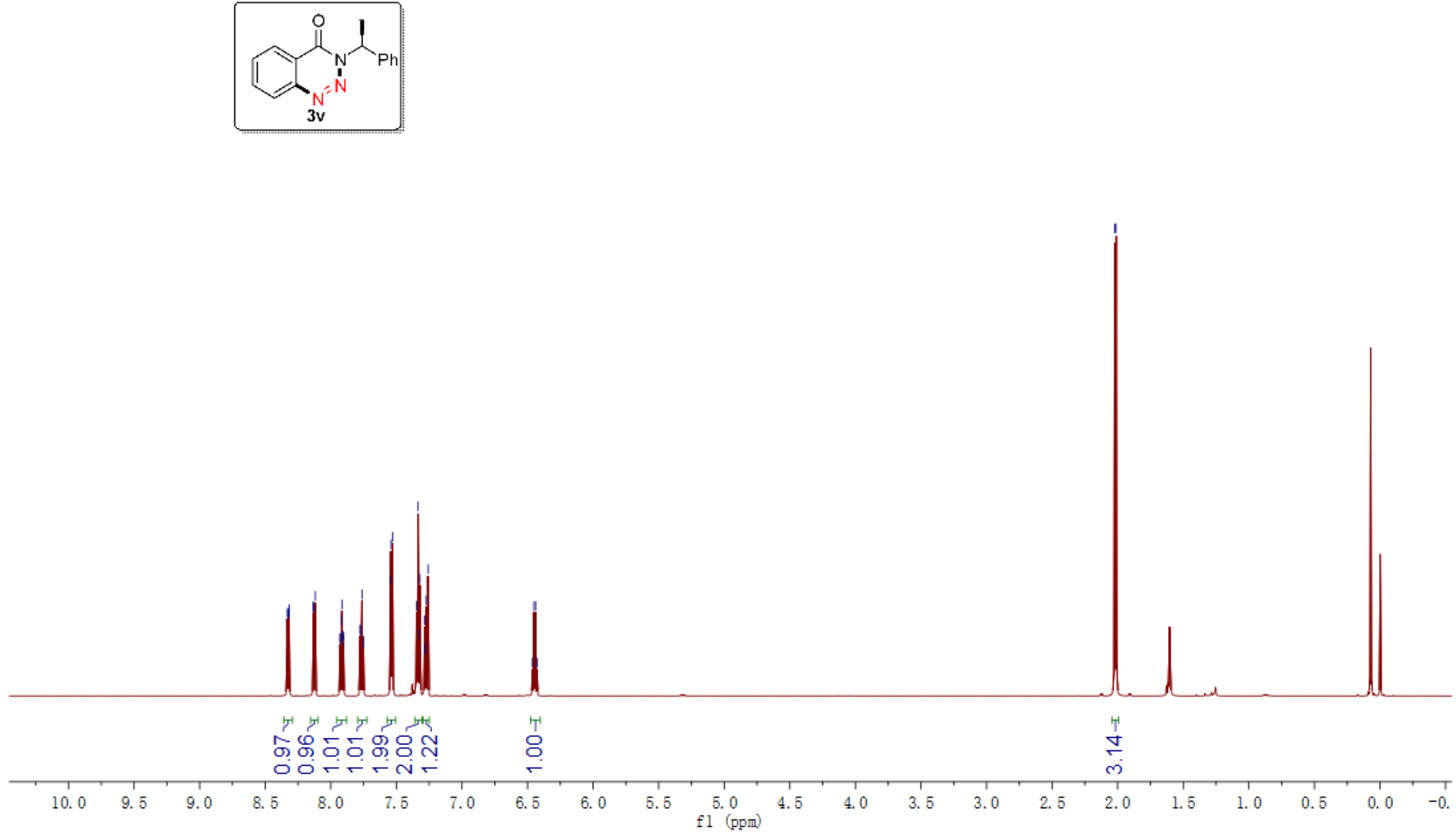
${ }^{13} \mathrm{C}$ - NMR spectrum of compound $\mathbf{3 v}\left(150 \mathrm{MHz}, \mathrm{CDCl}_{3}\right)$
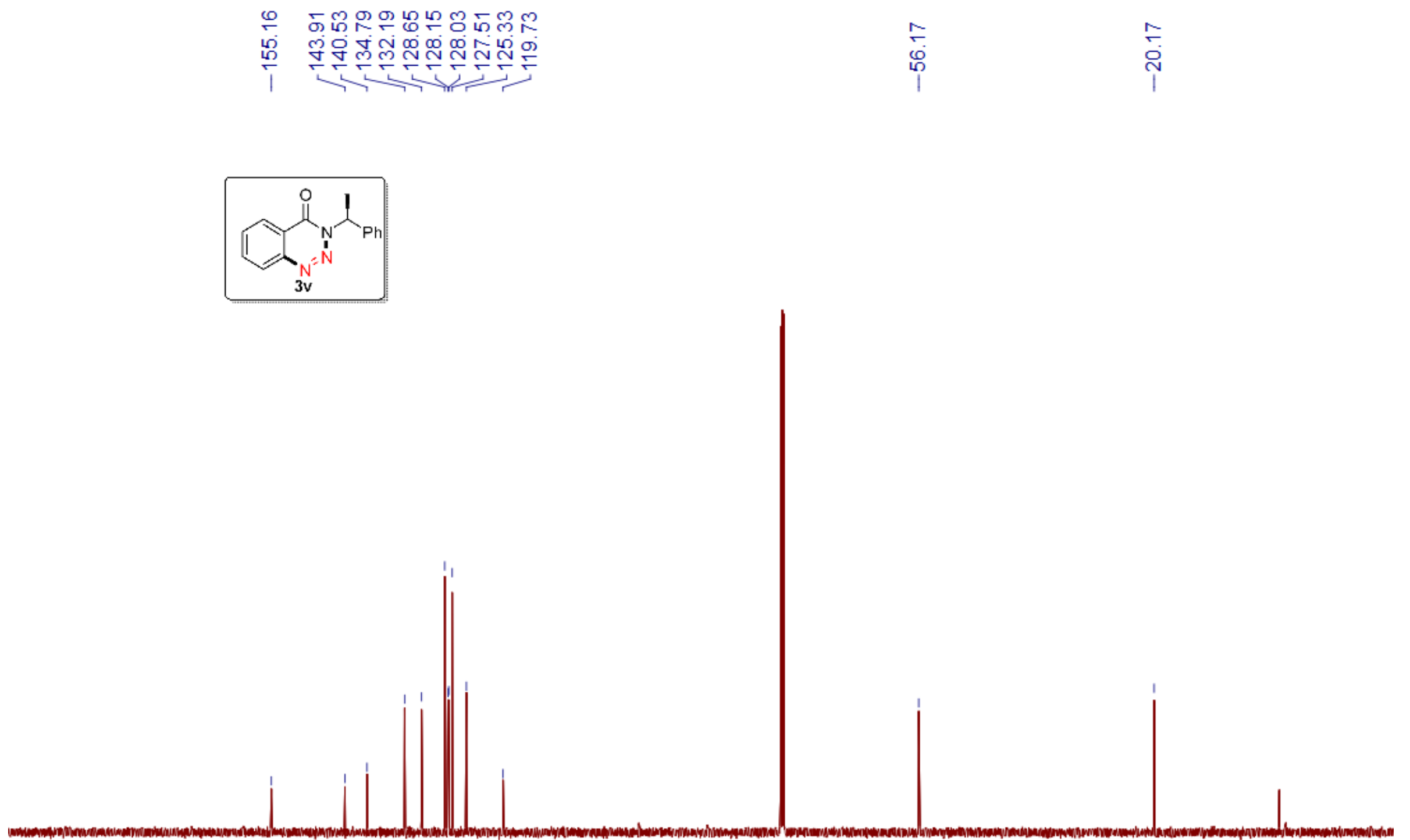
${ }^{1} \mathrm{H}$ - NMR spectrum of compound $\mathbf{3 w}\left(400 \mathrm{MHz}, \mathrm{CDCl}_{3}\right.$ )

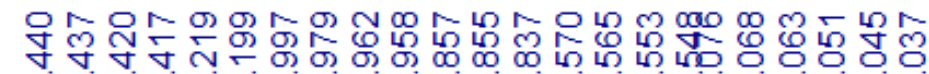

$\infty \infty \infty \infty \infty \infty \cdots+\cdots$
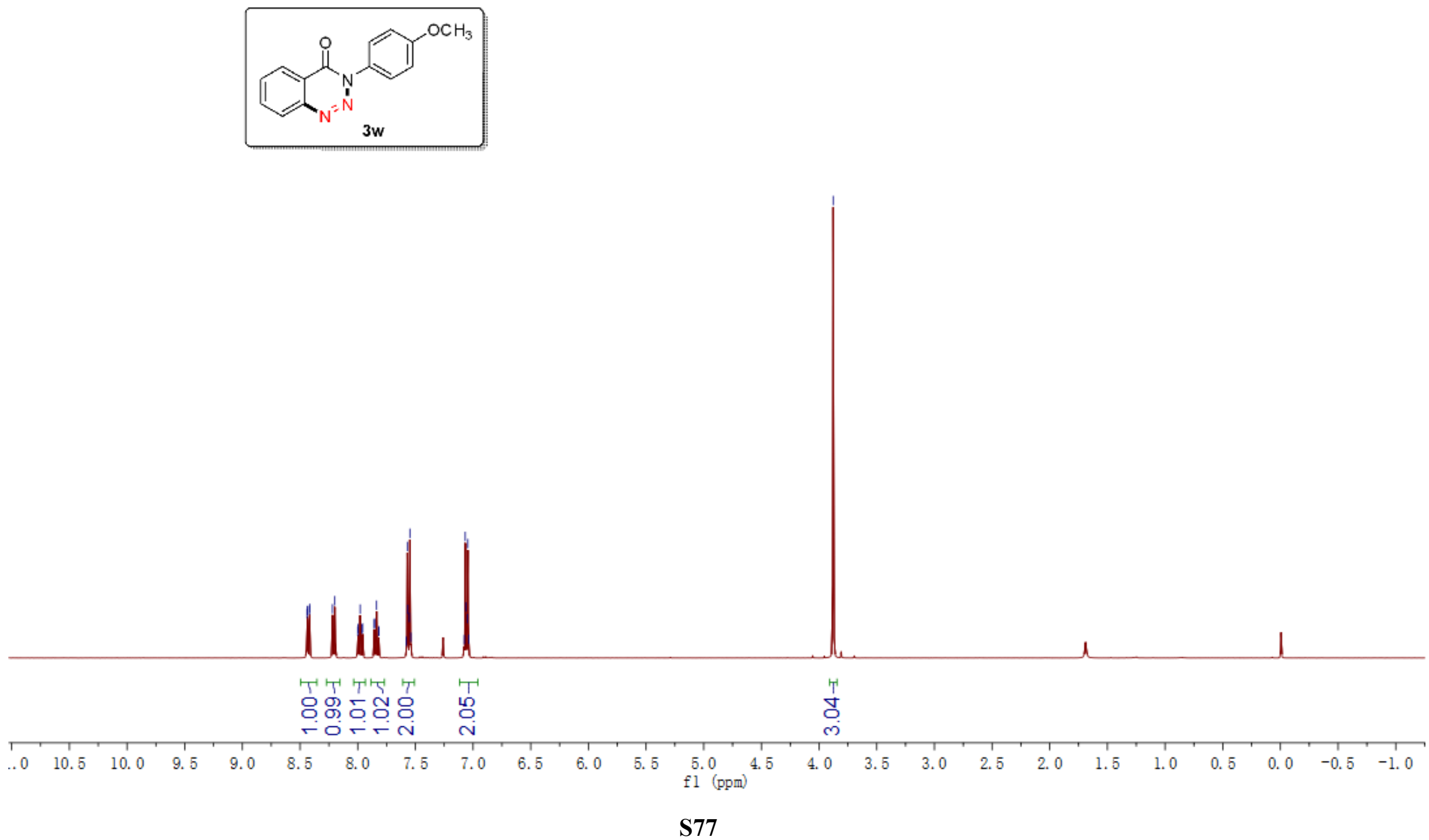
${ }^{13} \mathrm{C}$ - NMR spectrum of compound $\mathbf{3 w}\left(100 \mathrm{MHz}, \mathrm{CDCl}_{3}\right)$

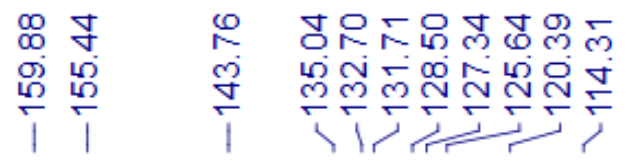

3
6
0
0
0
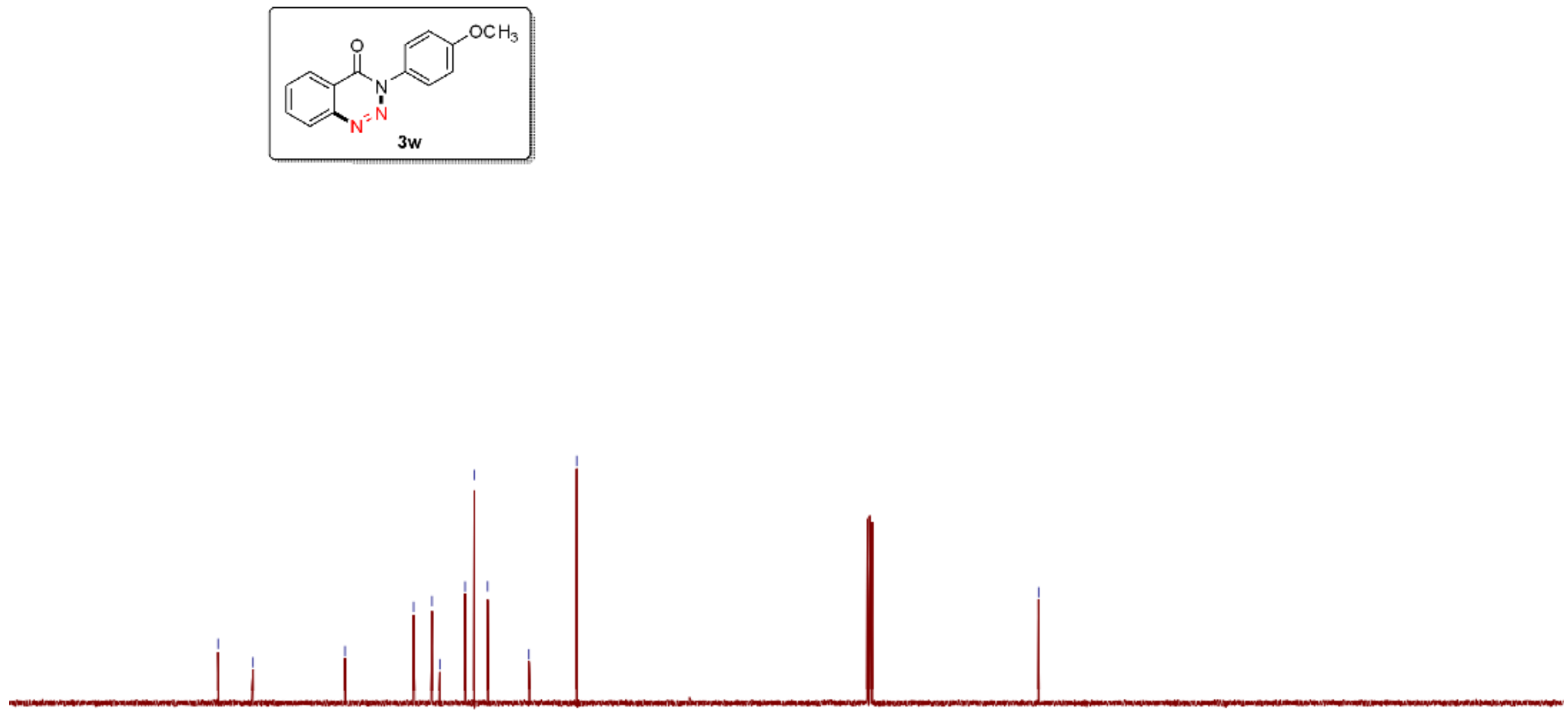

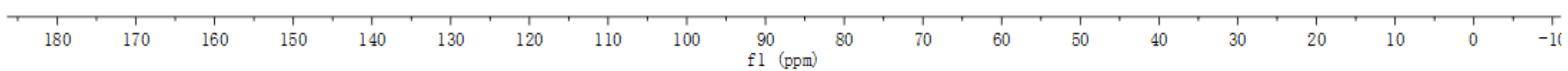


${ }^{1} \mathrm{H}$ - NMR spectrum of compound $\mathbf{3 x}\left(400 \mathrm{MHz}, \mathrm{CDCl}_{3}\right)$

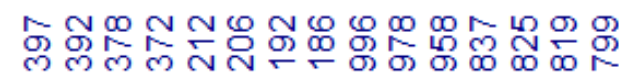

$\infty \infty \infty \infty \infty \infty \infty \cdots \infty \cdots$

$\stackrel{\infty}{\stackrel{\infty}{\infty}}$

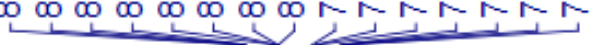
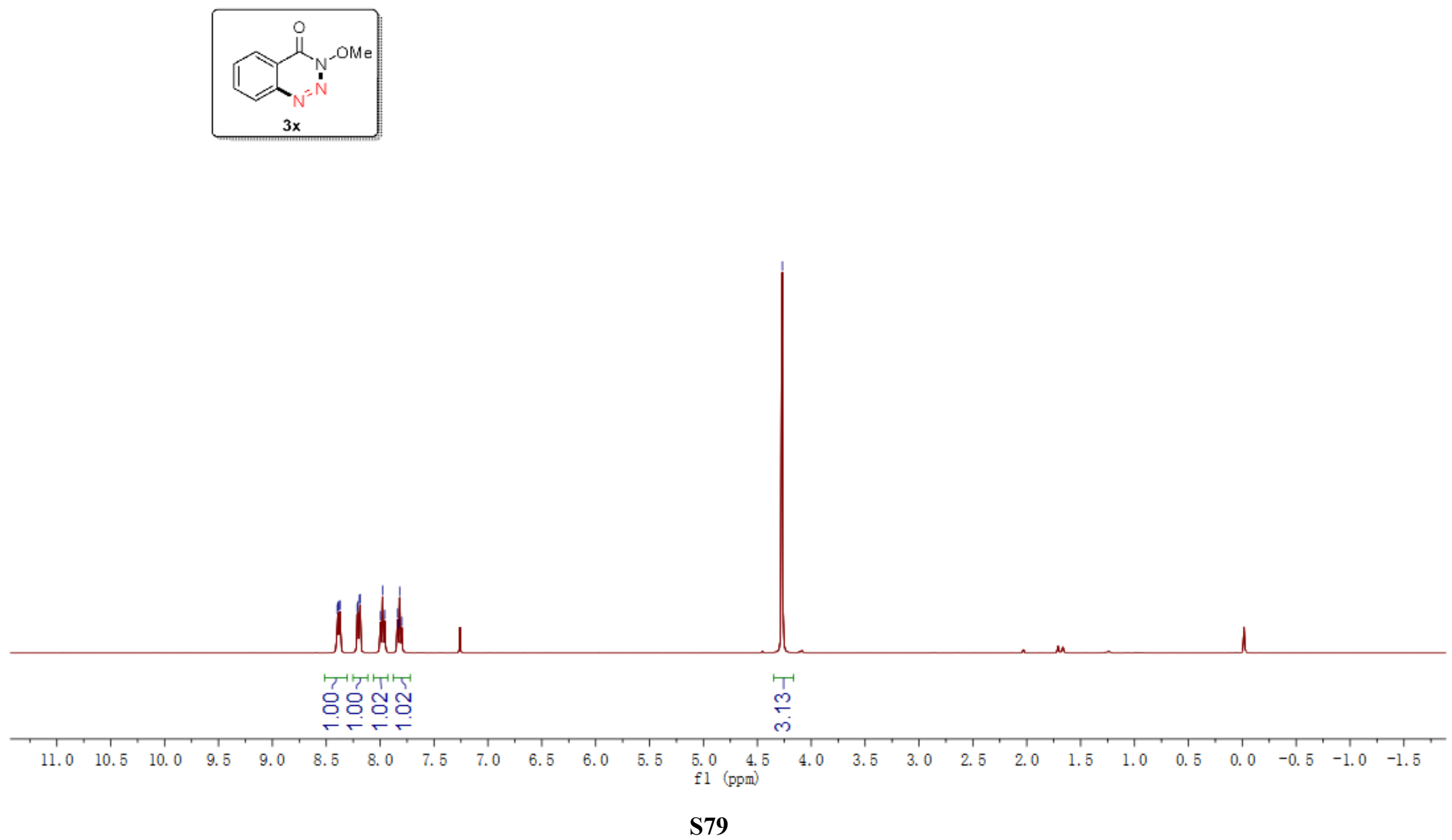
${ }^{13} \mathrm{C}$ - NMR spectrum of compound $\mathbf{3 x}\left(125 \mathrm{MHz}, \mathrm{CDCl}_{3}\right)$

$$
\text { i }
$$

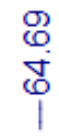
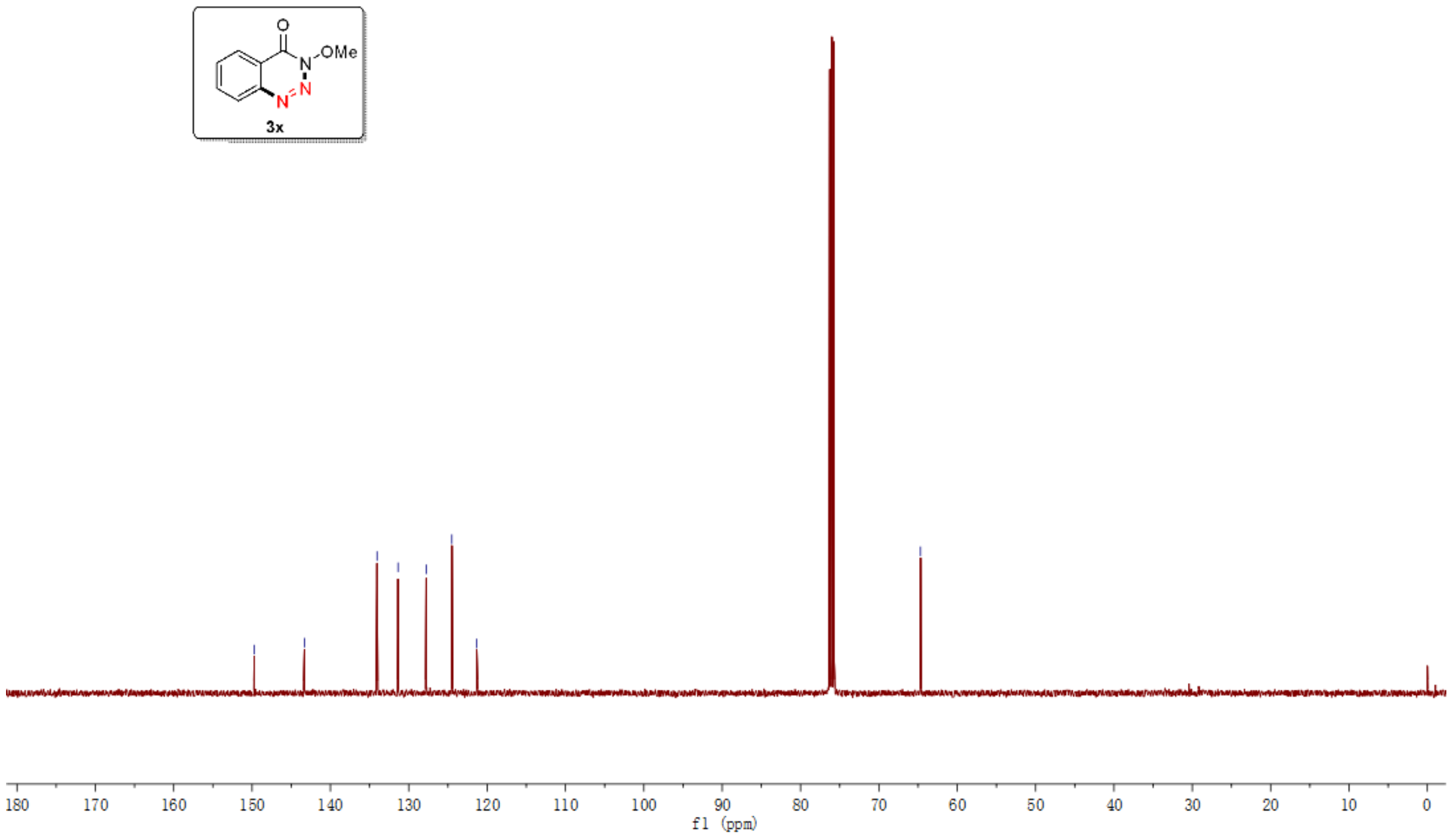

S80 
${ }^{1} \mathrm{H}$ - NMR spectrum of compound $\mathbf{5 a}\left(400 \mathrm{MHz}, \mathrm{CDCl}_{3}\right)$

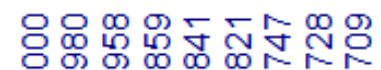

$\infty N \cdots N$

L
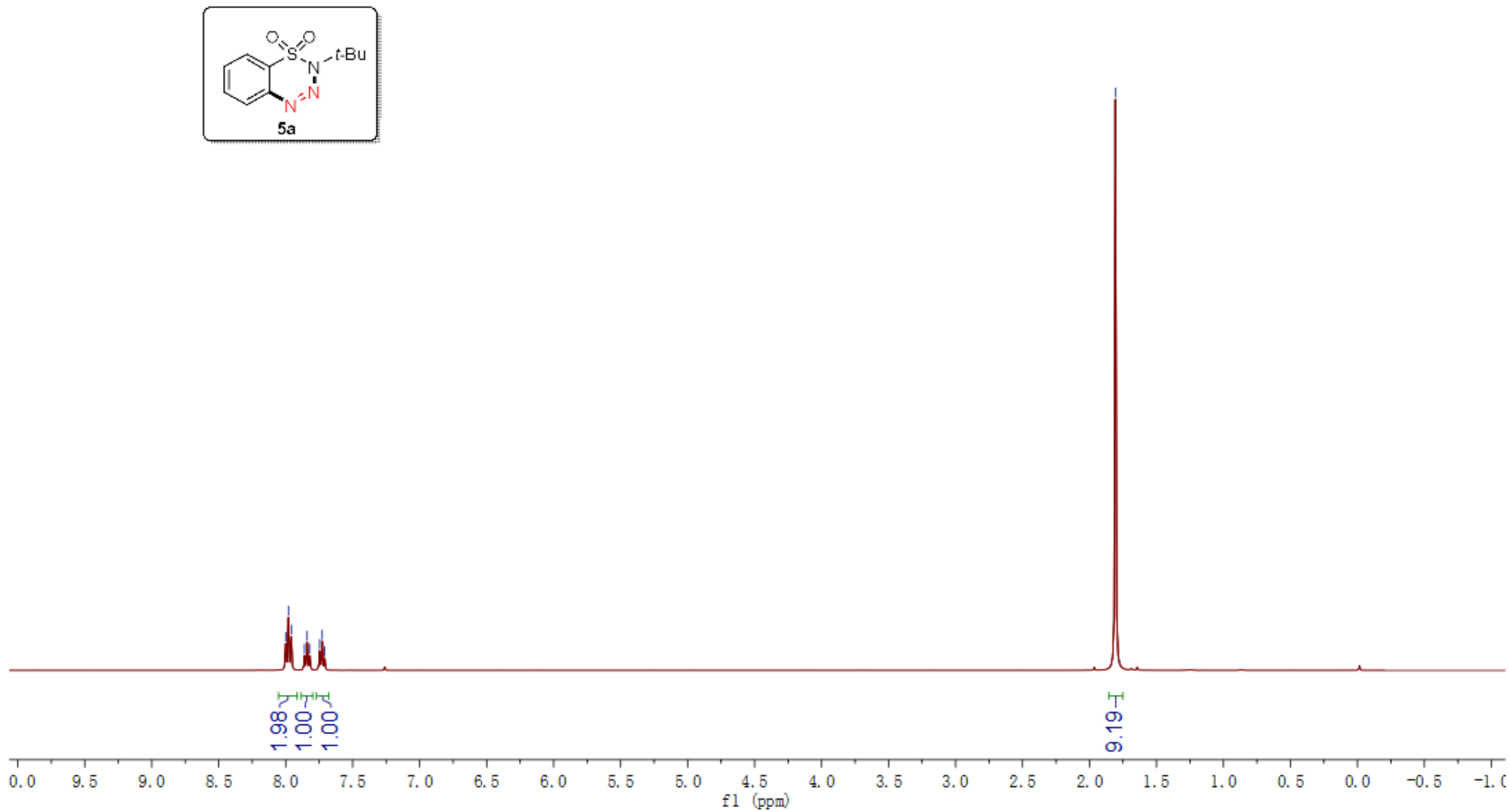
${ }^{13} \mathrm{C}$ - NMR spectrum of compound $\mathbf{5 a}\left(100 \mathrm{MHz}, \mathrm{CDCl}_{3}\right)$
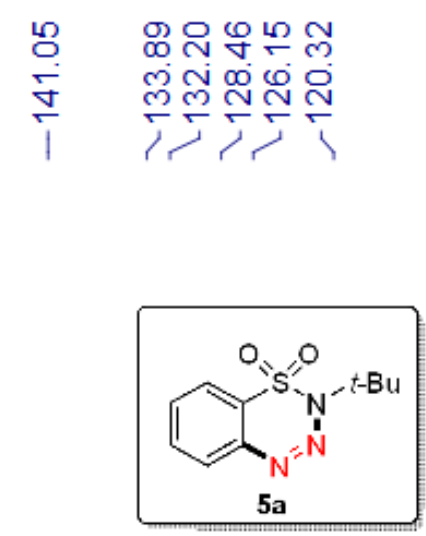

$\|\left.\right|_{1}$

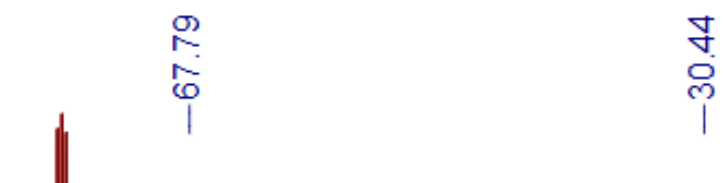

守

Dits

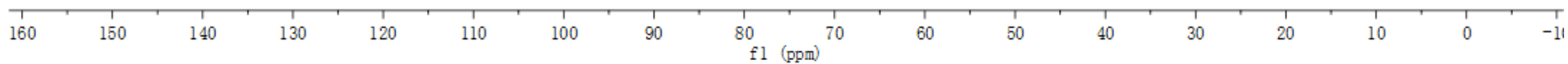


${ }^{1} \mathrm{H}$ - NMR spectrum of compound $\mathbf{5 b}\left(400 \mathrm{MHz}, \mathrm{CDCl}_{3}\right)$

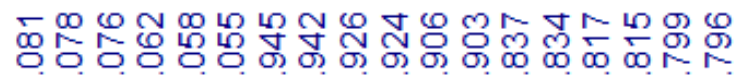

$\infty \infty \infty \infty \infty \infty N+N)$
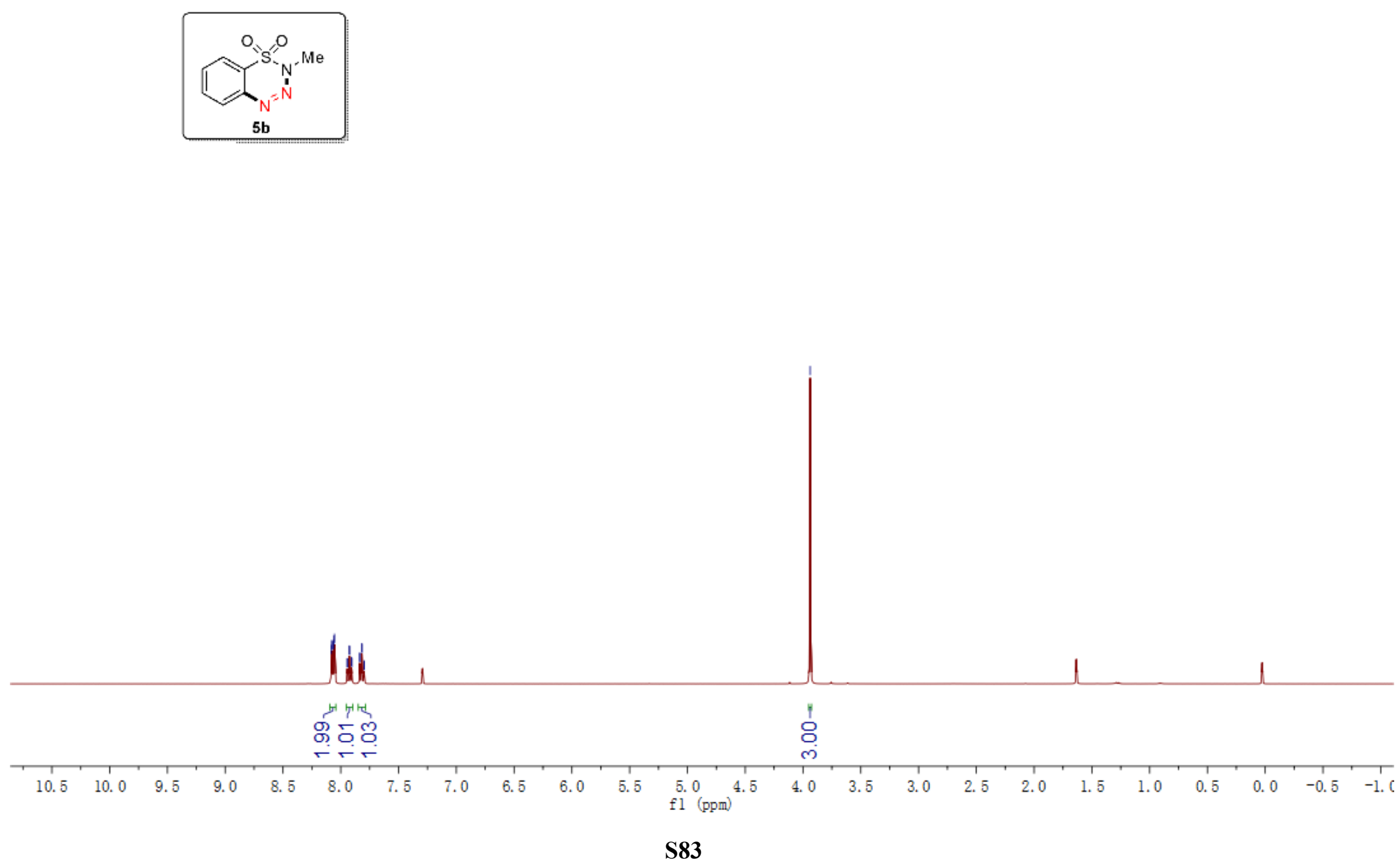
${ }^{13} \mathrm{C}$ - NMR spectrum of compound $\mathbf{5 b}\left(100 \mathrm{MHz}, \mathrm{CDCl}_{3}\right)$

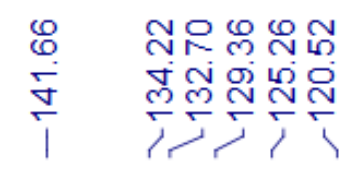

$\hat{\infty}$

$m$
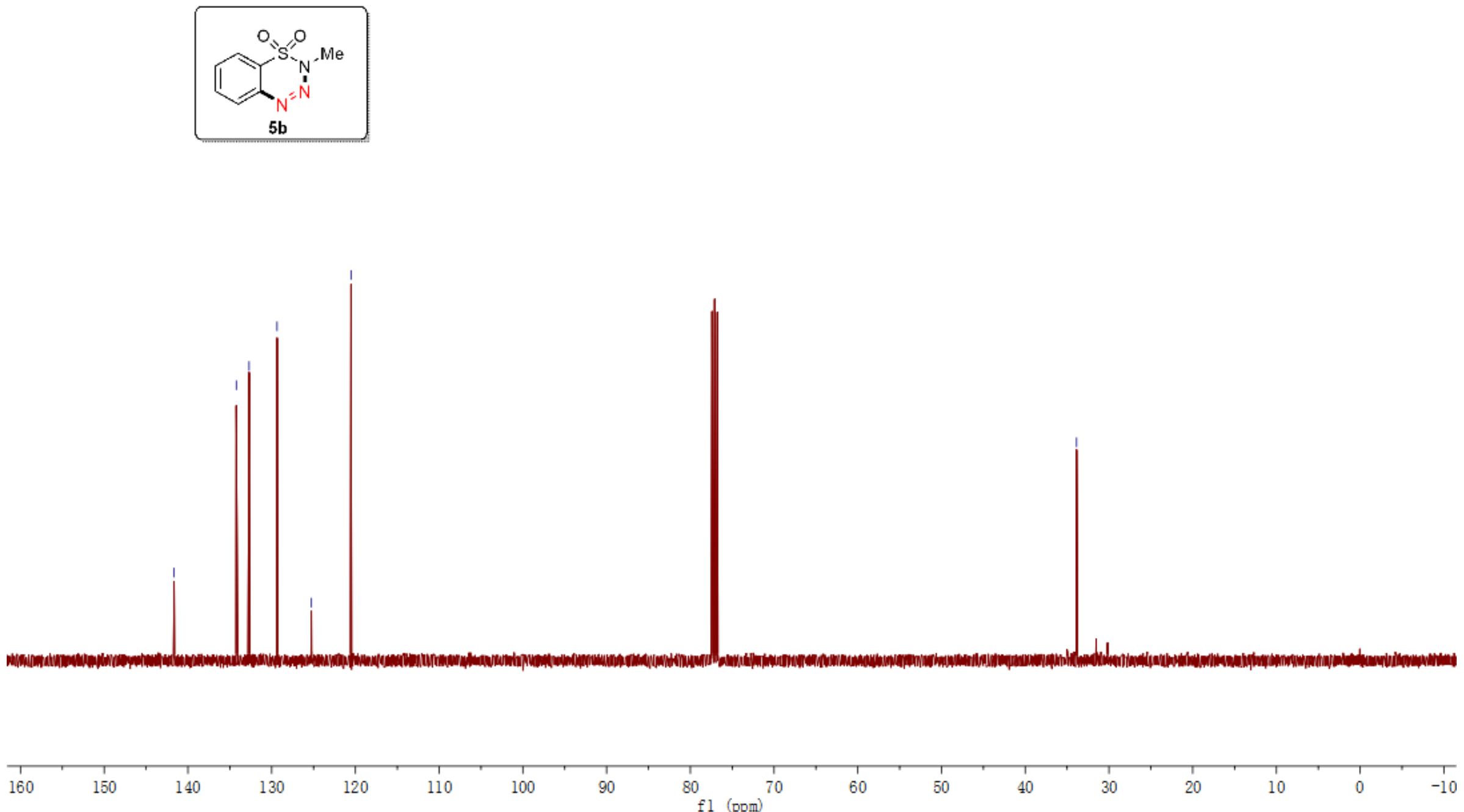
${ }^{1} \mathrm{H}$ - NMR spectrum of compound $\mathbf{5 c}\left(400 \mathrm{MHz}, \mathrm{CDCl}_{3}\right)$

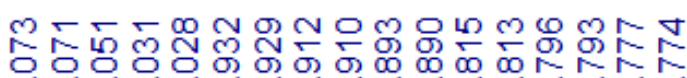

$\infty \infty \infty \infty \cdots \cdots N-N$

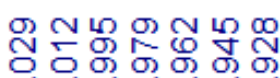

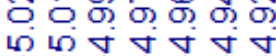

离品

$\sqrt{0.010}$
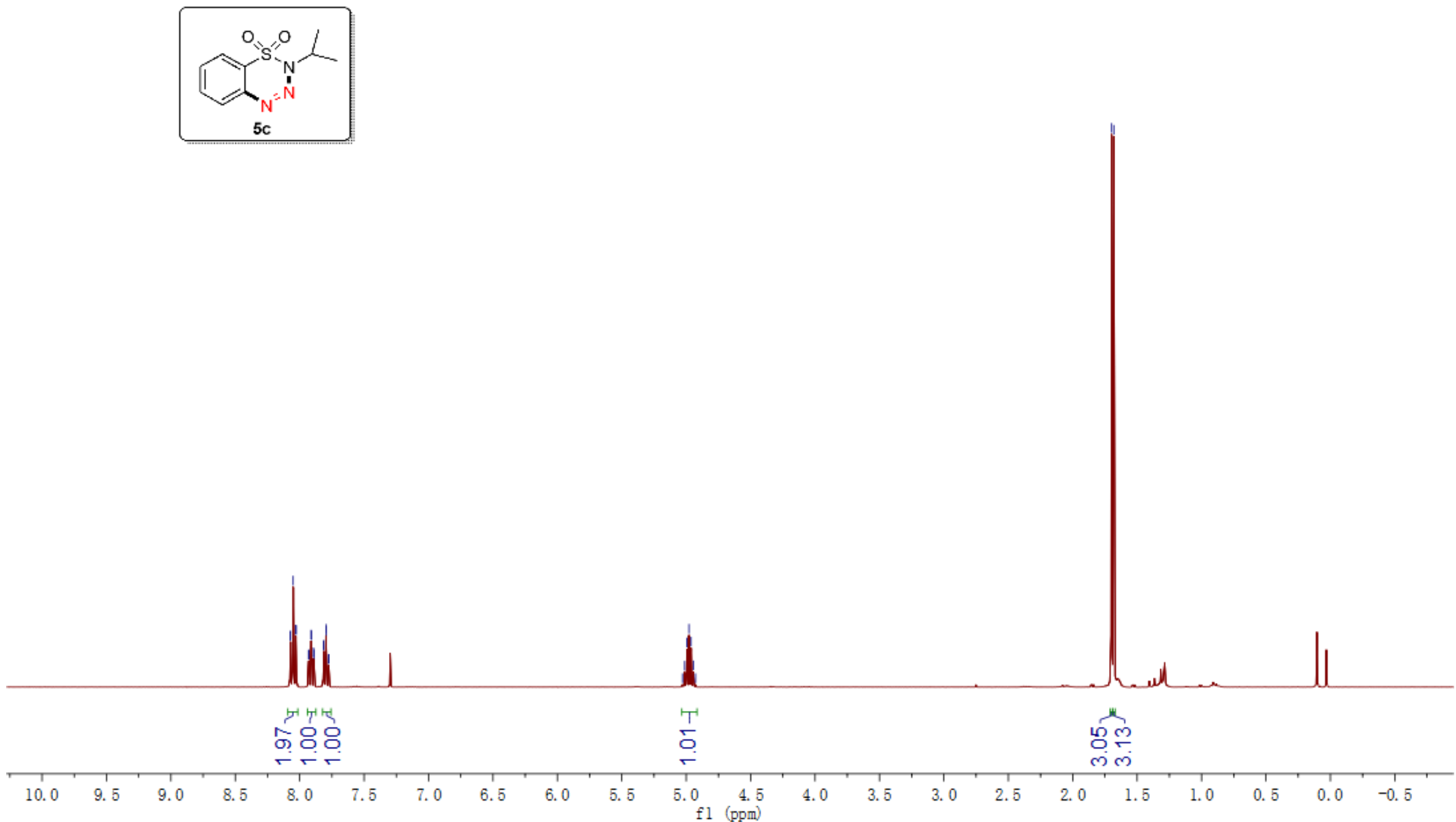
${ }^{13} \mathrm{C}$ - NMR spectrum of compound $\mathbf{5 c}\left(100 \mathrm{MHz}, \mathrm{CDCl}_{3}\right)$

$$
\text { i }
$$

$\stackrel{\oplus}{\circ}$

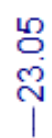

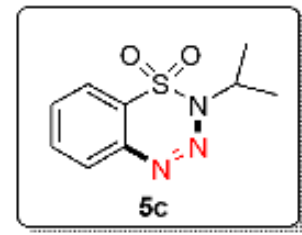

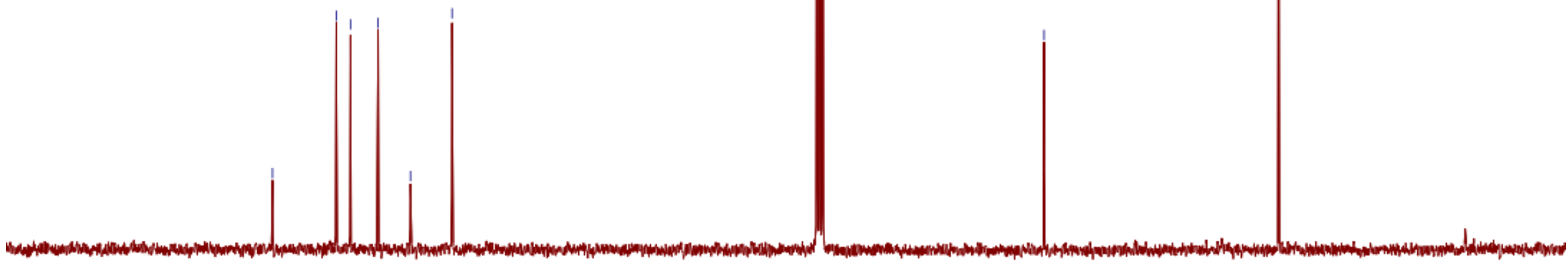

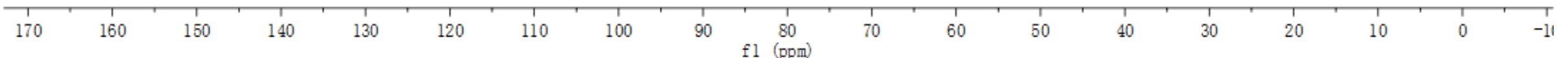


${ }^{1} \mathrm{H}$ - NMR spectrum of compound $\mathbf{5 d}\left(400 \mathrm{MHz}, \mathrm{CDCl}_{3}\right)$

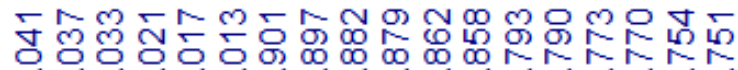

$\infty \infty \infty \infty \infty \infty \cdots$

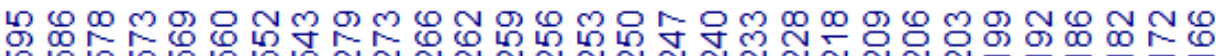

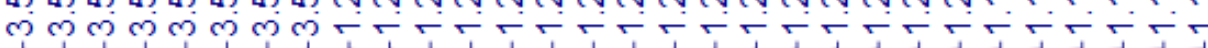
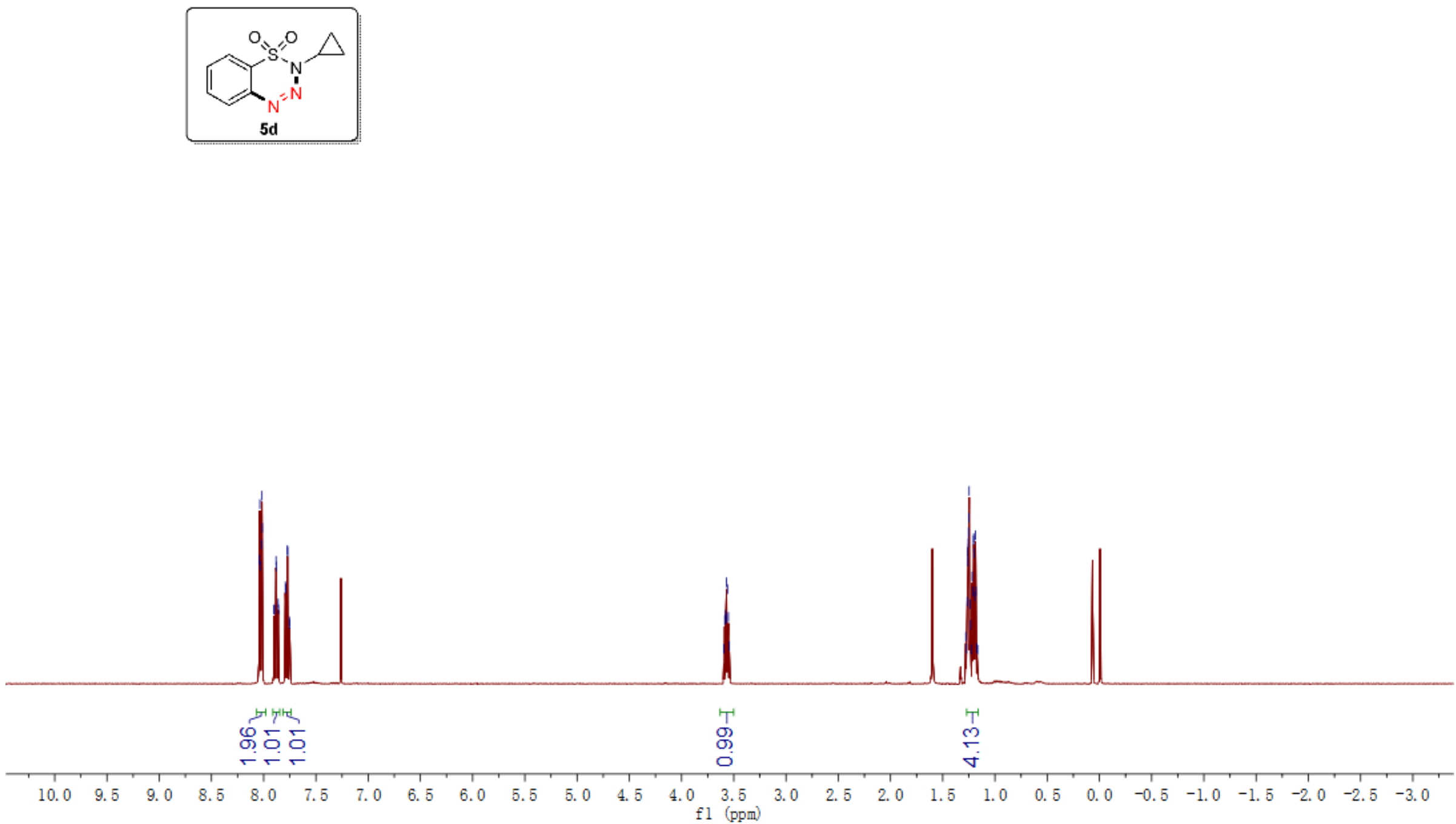
${ }^{13} \mathrm{C}$ - NMR spectrum of compound $\mathbf{5 d}\left(100 \mathrm{MHz}, \mathrm{CDCl}_{3}\right)$
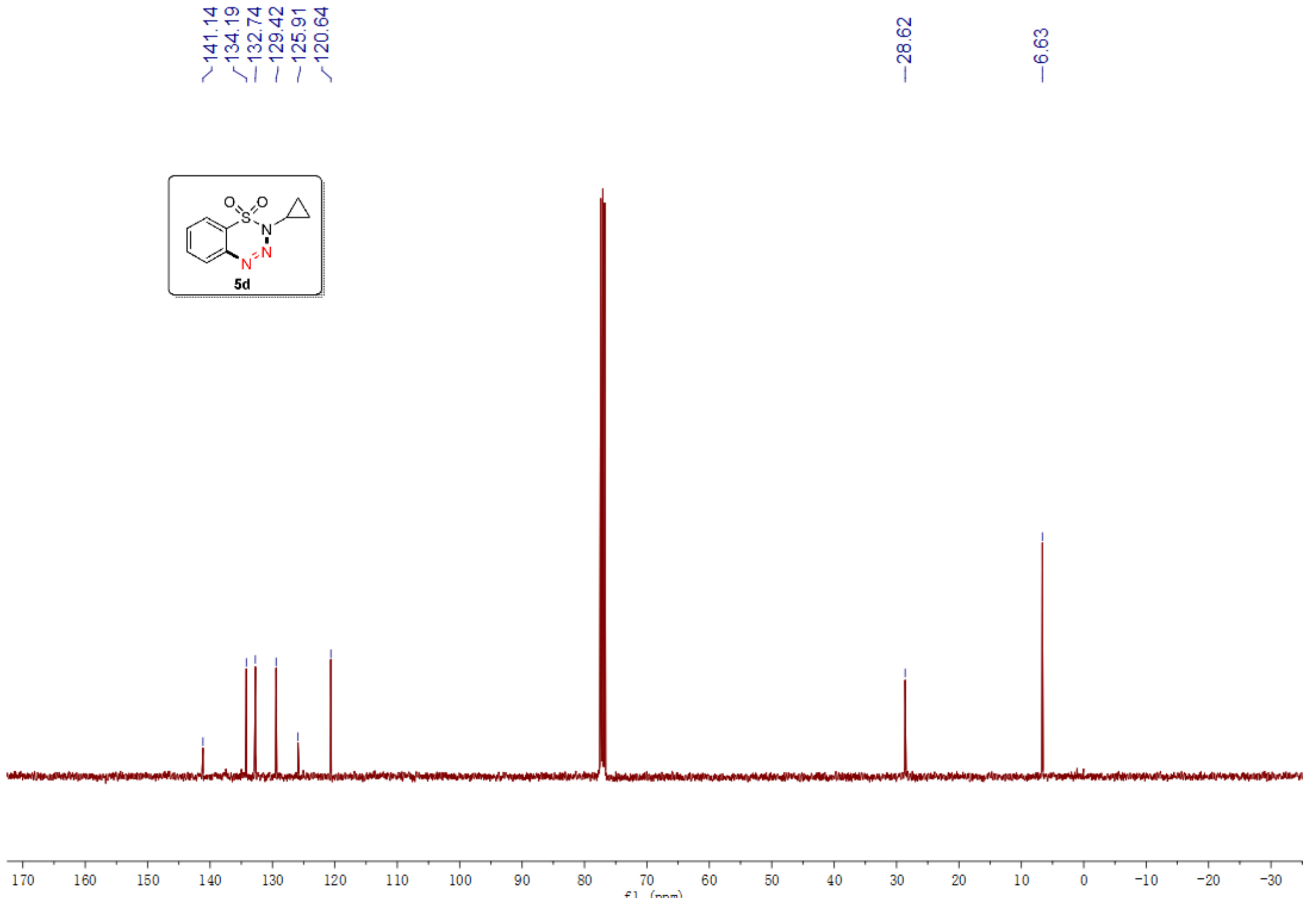

fl ${ }^{70}(\mathrm{ppm})$

1
60
50


${ }^{1} \mathrm{H}$ - NMR spectrum of compound $\mathbf{5 e}\left(400 \mathrm{MHz}, \mathrm{CDCl}_{3}\right)$

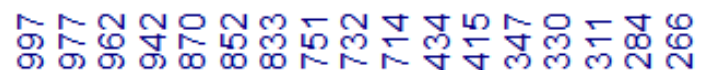

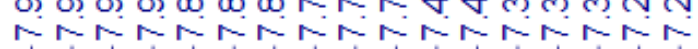
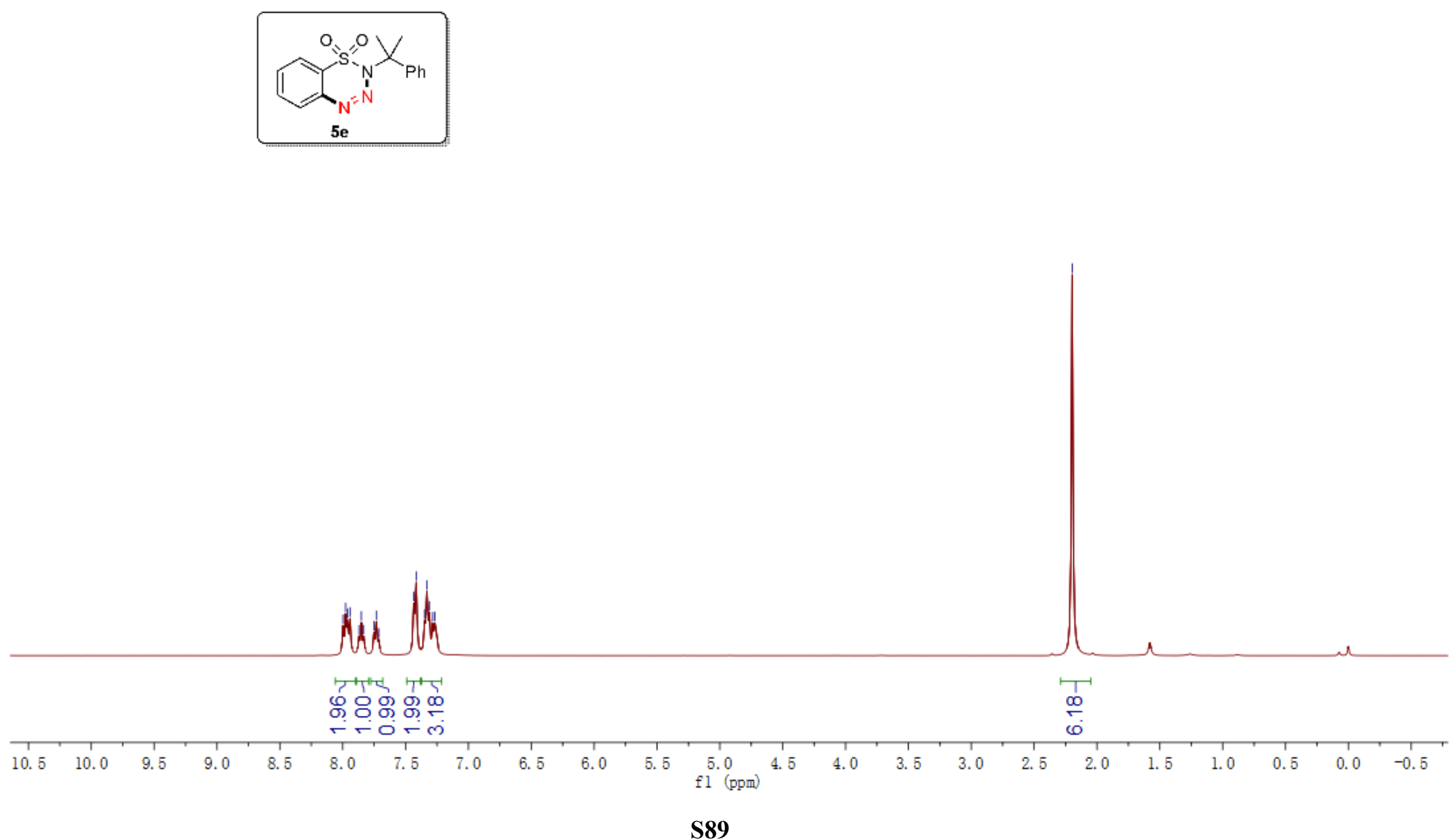
${ }^{13} \mathrm{C}$ - NMR spectrum of compound $\mathbf{5 e}\left(100 \mathrm{MHz}, \mathrm{CDCl}_{3}\right)$

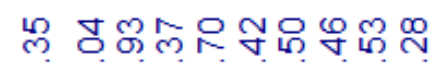

守

$\stackrel{\substack{\infty \\ \stackrel{\infty}{P}}}{\stackrel{n}{0}}$
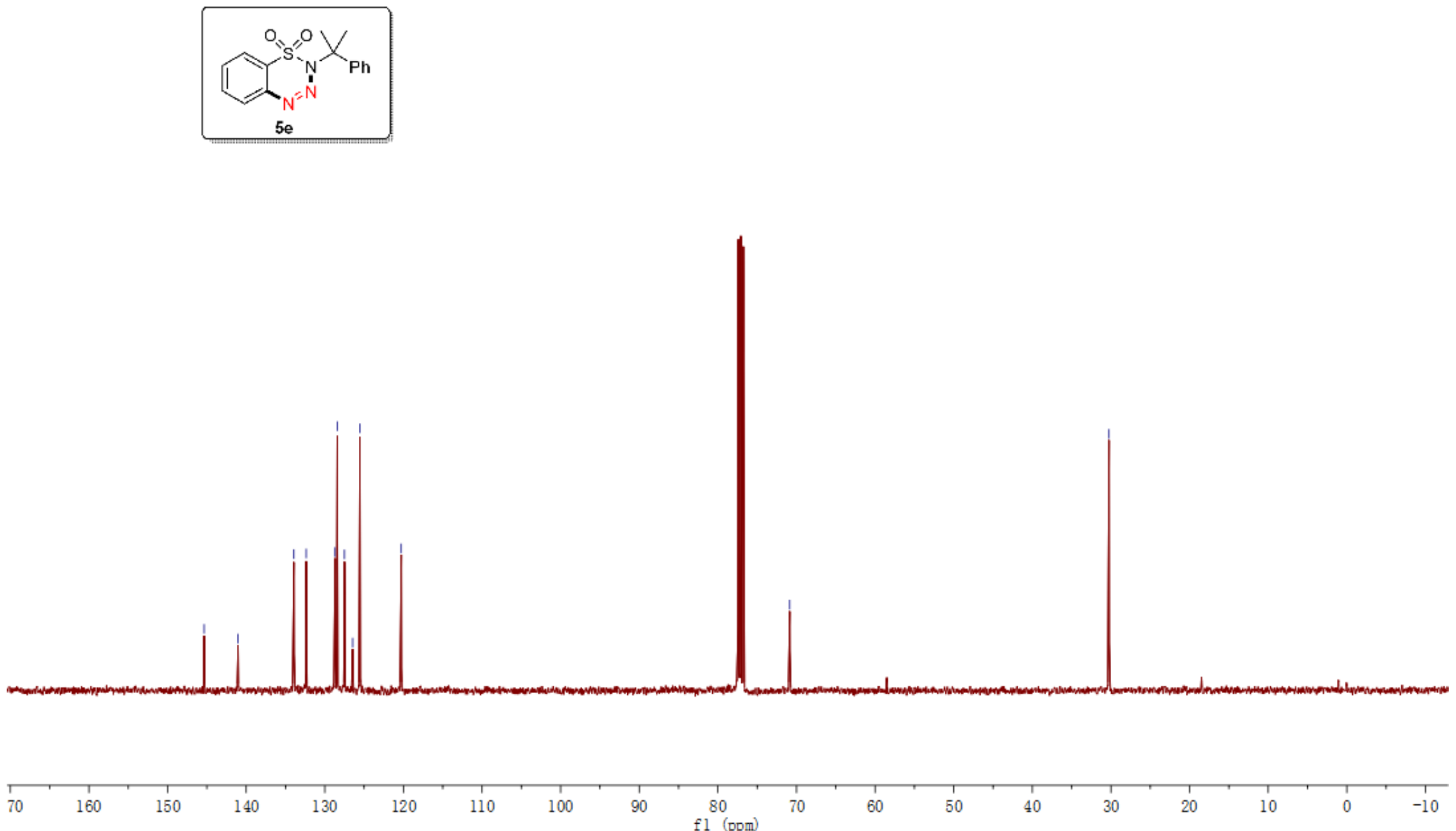
${ }^{1} \mathrm{H}$ - NMR spectrum of compound $\mathbf{5 f}\left(400 \mathrm{MHz}, \mathrm{CDCl}_{3}\right)$

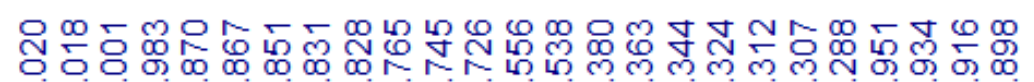

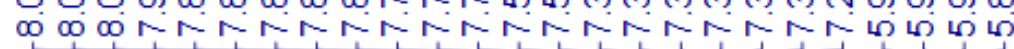

$\stackrel{\infty}{\circ} \stackrel{0}{\circ}$

Ni

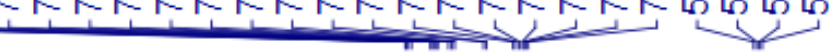
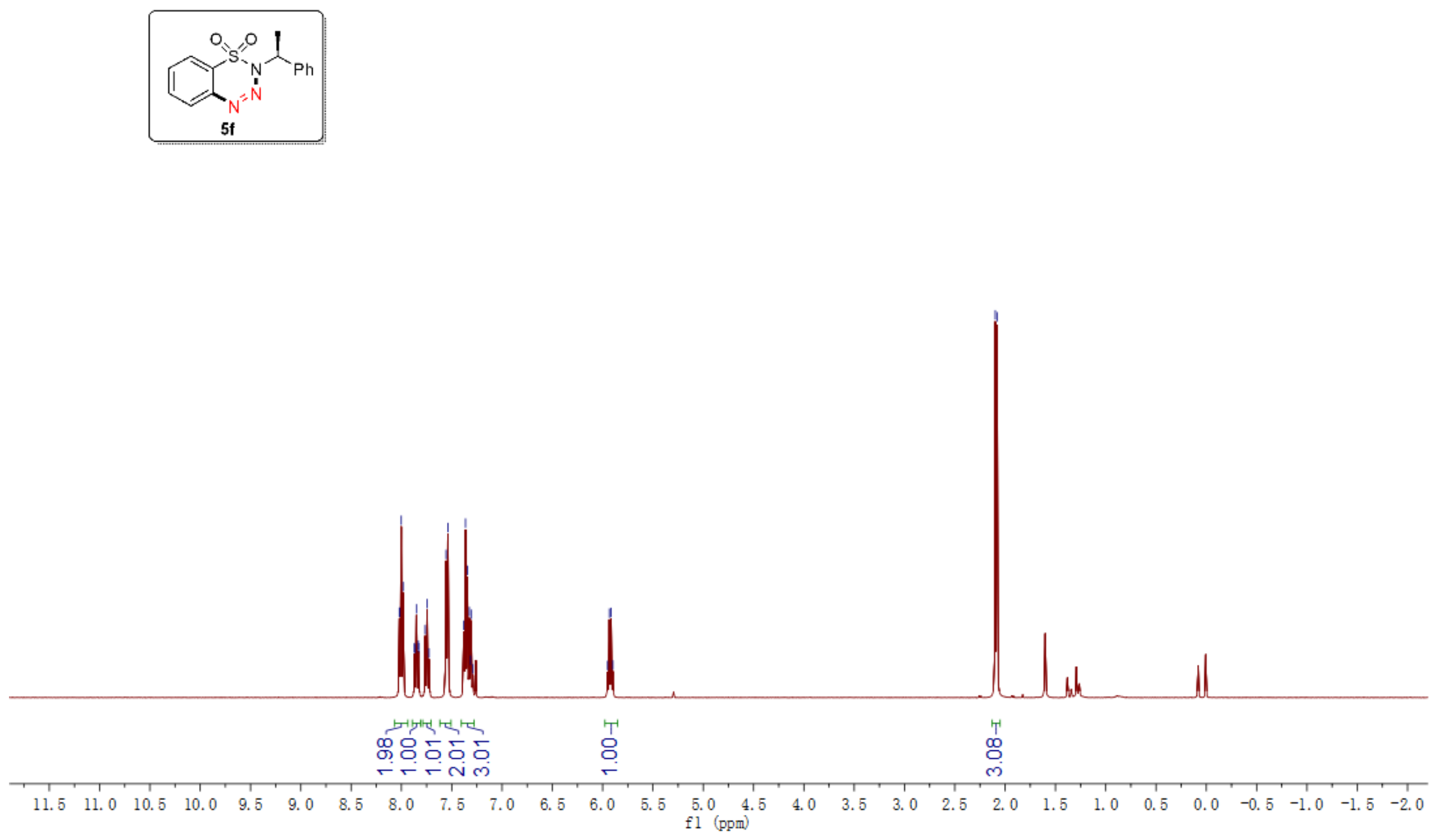
${ }^{13} \mathrm{C}$ - NMR spectrum of compound $\mathbf{5 f}\left(100 \mathrm{MHz}, \mathrm{CDCl}_{3}\right)$

垨

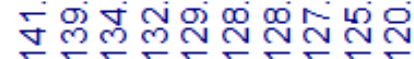

怘

i

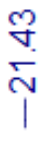
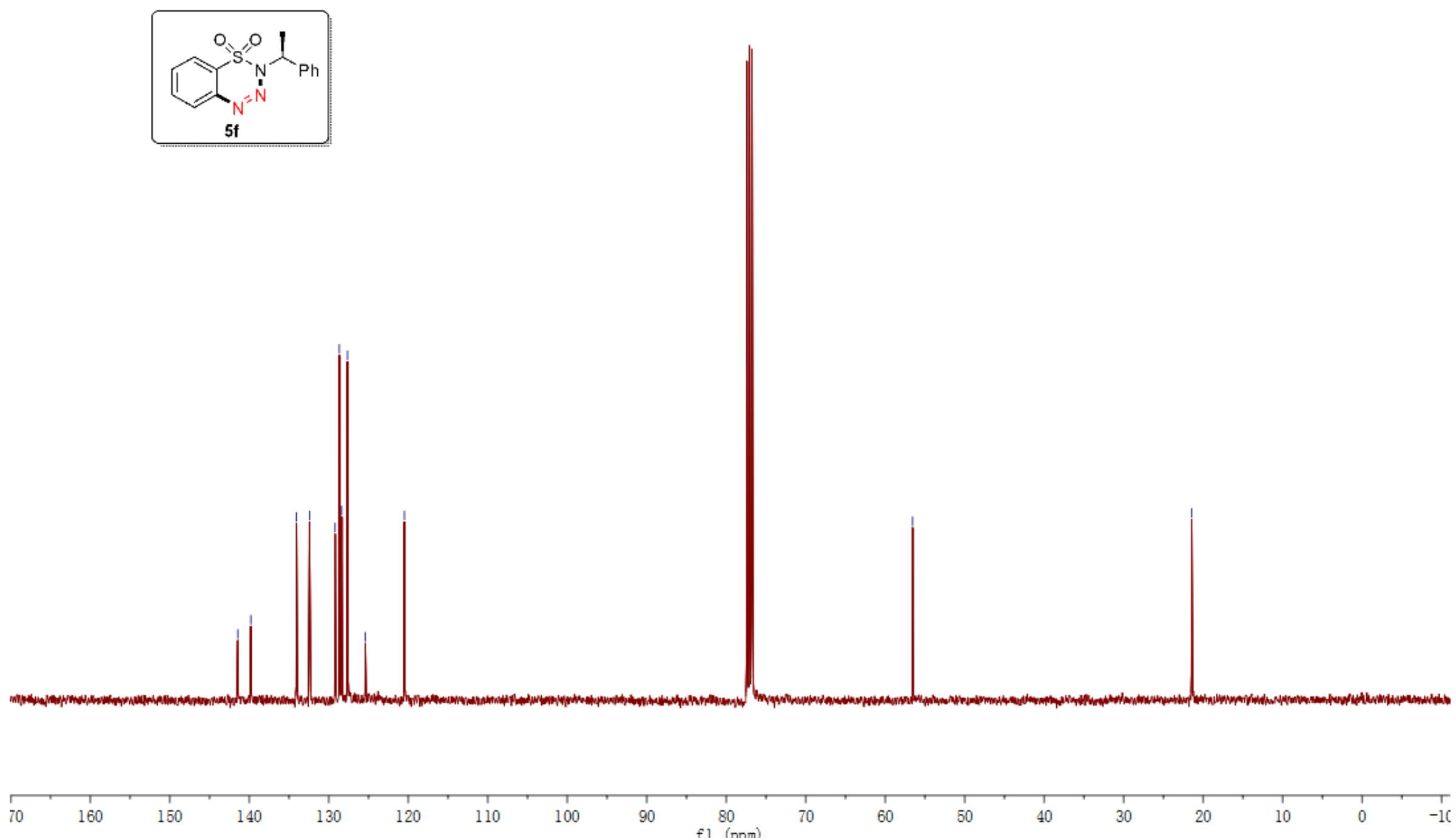

fl 80

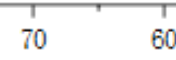

60

50

40

30

20

10

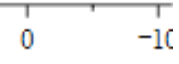


${ }^{1} \mathrm{H}$ - NMR spectrum of compound $\mathbf{5 g}\left(400 \mathrm{MHz}, \mathrm{CDCl}_{3}\right)$

$$
\begin{aligned}
& \text { 只 N } \\
& \text { 勿! }
\end{aligned}
$$
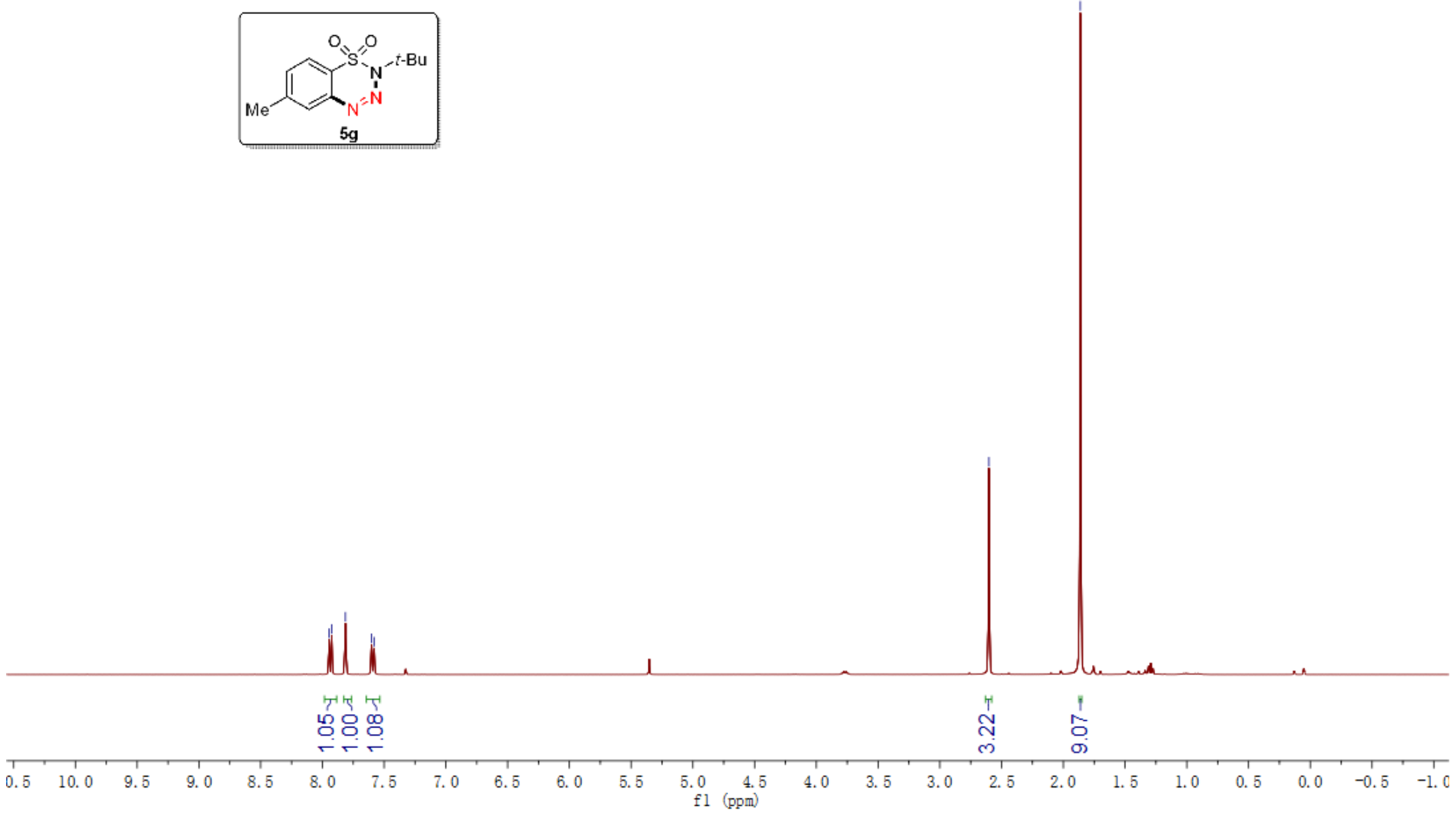
${ }^{13} \mathrm{C}$ - NMR spectrum of compound $\mathbf{5 g}\left(100 \mathrm{MHz}, \mathrm{CDCl}_{3}\right)$

官

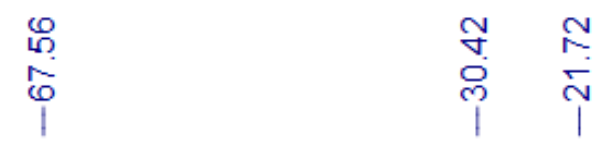
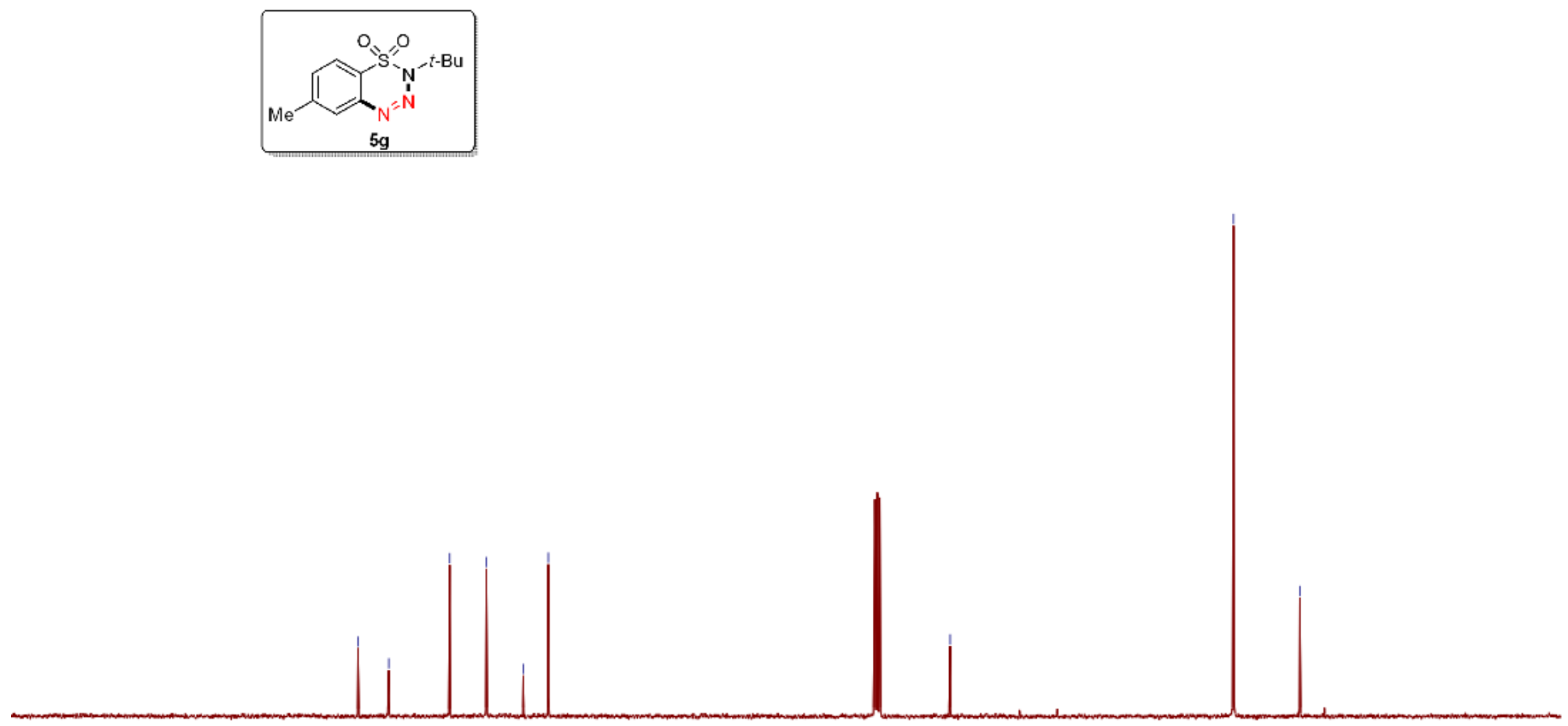

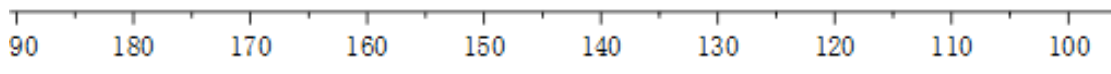

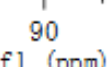

$80 \quad 70$

60

50

40

30

20 
${ }^{1} \mathrm{H}$ - NMR spectrum of compound $\mathbf{5 h}\left(400 \mathrm{MHz}, \mathrm{CDCl}_{3}\right)$

ֻ

NN

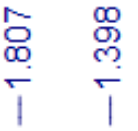
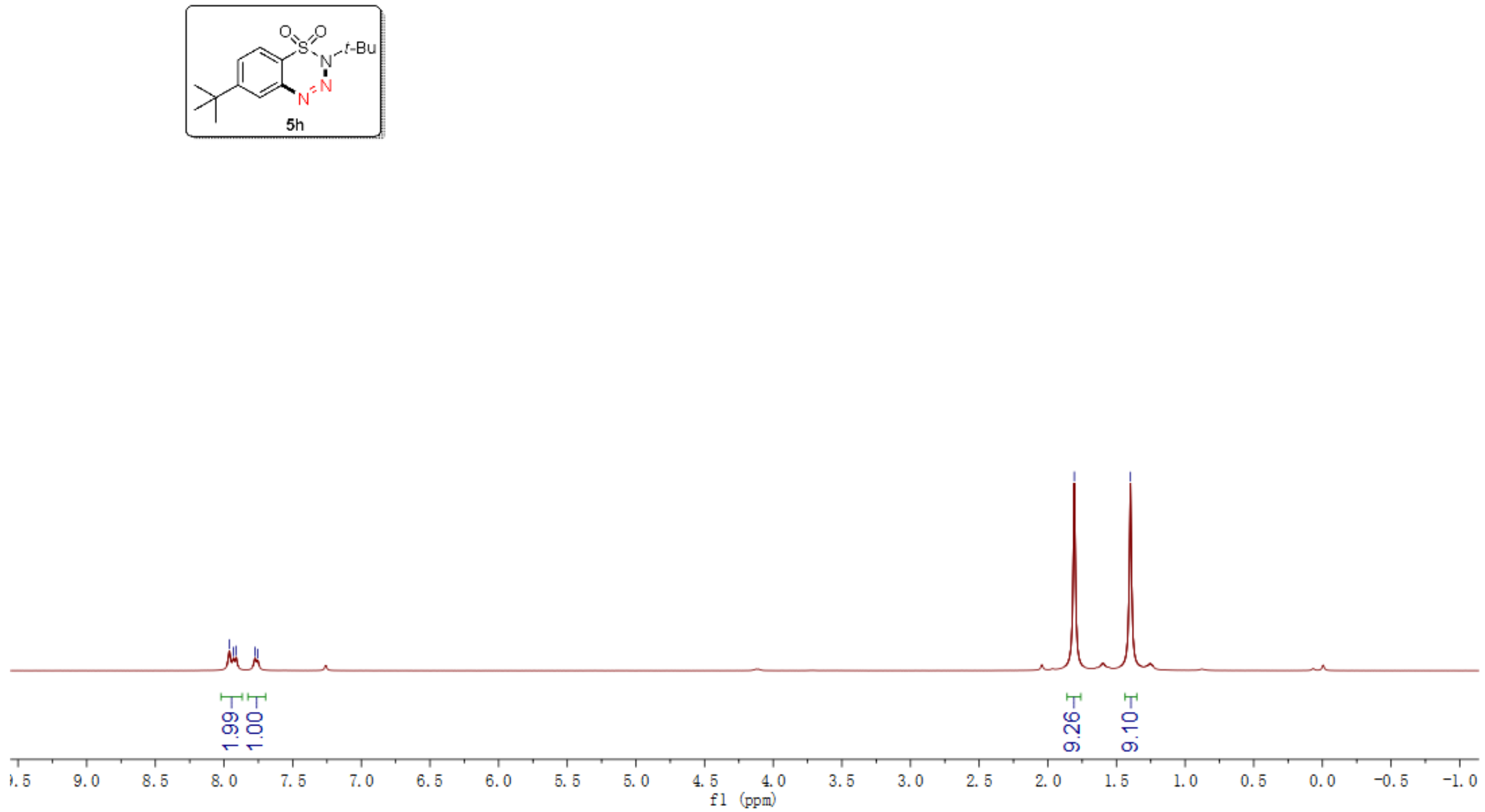
${ }^{13} \mathrm{C}$ - NMR spectrum of compound $\mathbf{5 h}\left(100 \mathrm{MHz}, \mathrm{CDCl}_{3}\right)$

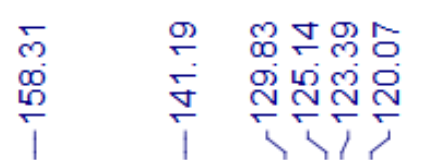
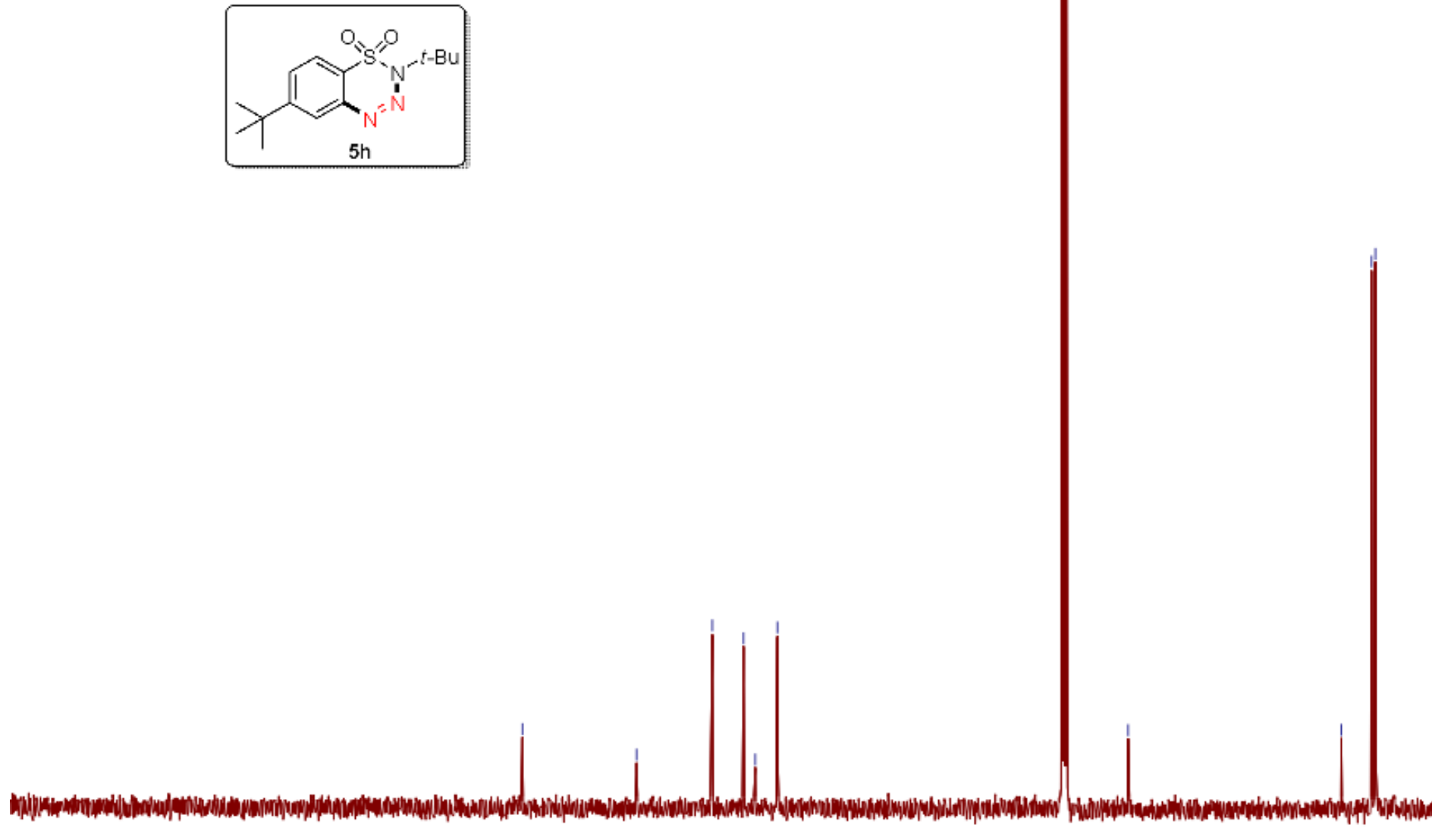

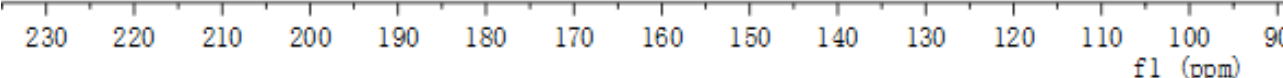

80

06

40

20

$\begin{array}{lll}-10 & -20 & -30\end{array}$ 
${ }^{1} \mathrm{H}$ - NMR spectrum of compound $\mathbf{5 i}\left(400 \mathrm{MHz}, \mathrm{CDCl}_{3}\right)$

மூ:

$\infty \infty \infty \cdots N-N$
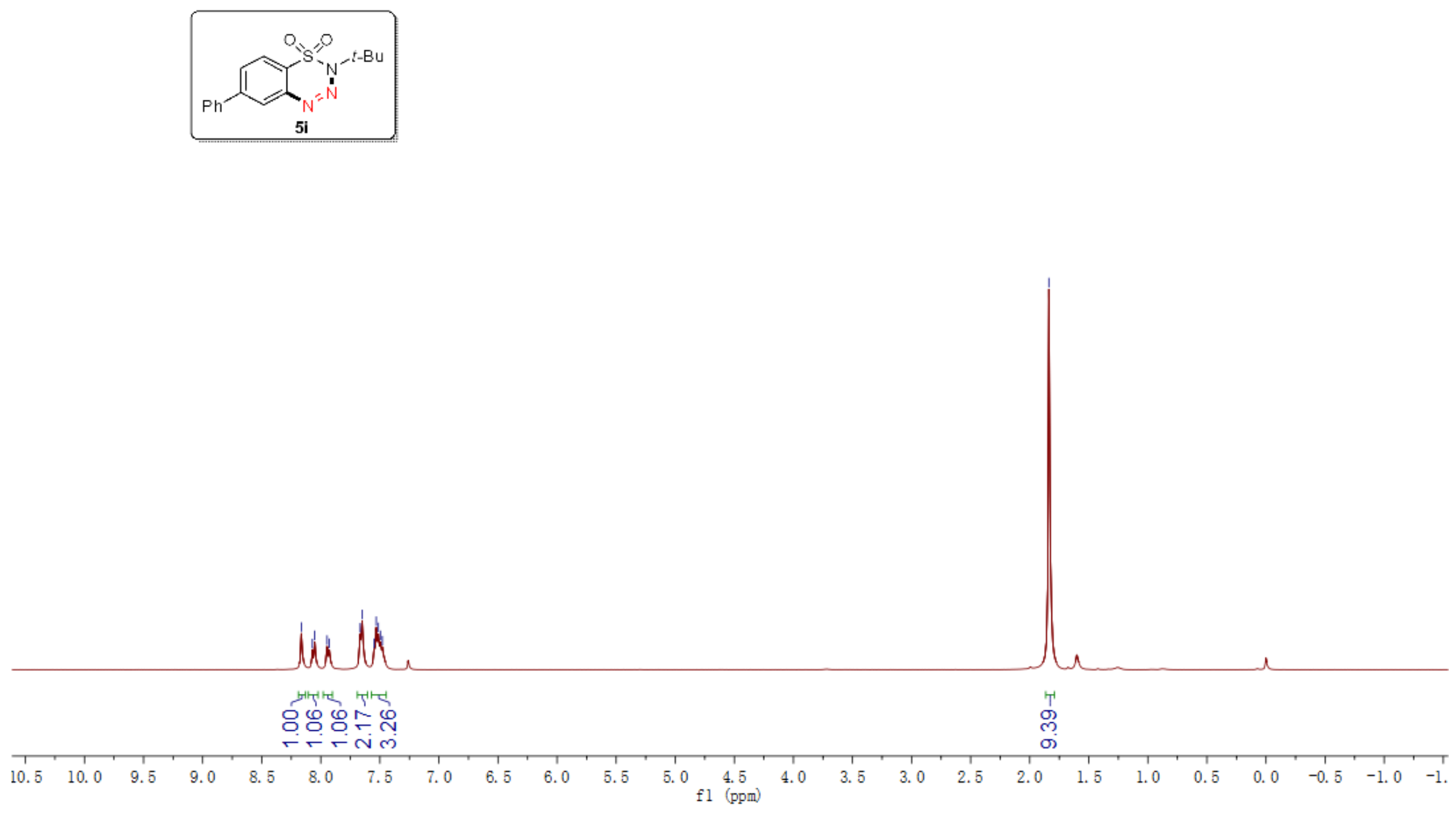
${ }^{13} \mathrm{C}$ - NMR spectrum of compound $\mathbf{5 i}\left(100 \mathrm{MHz}, \mathrm{CDCl}_{3}\right)$

ำ

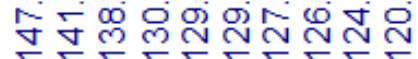

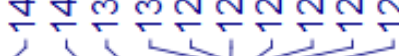

\begin{tabular}{ll}
$m$ & \multirow{5}{*}{} \\
0 & 0 \\
0 & 0
\end{tabular}
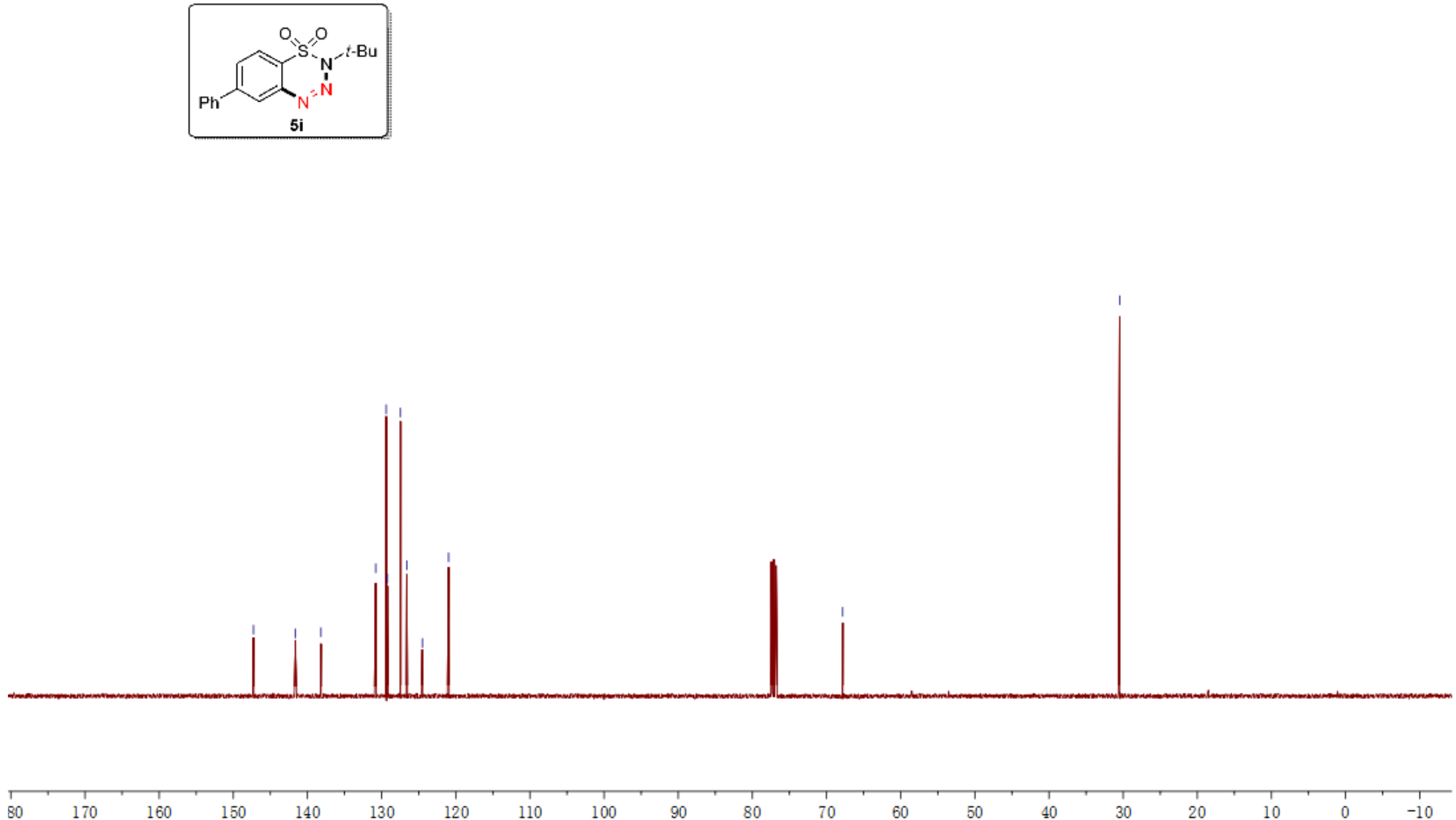

120

110 
${ }^{1} \mathrm{H}$ - NMR spectrum of compound $\mathbf{5 j}\left(600 \mathrm{MHz}, \mathrm{CDCl}_{3}\right)$

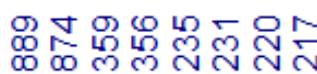

minn

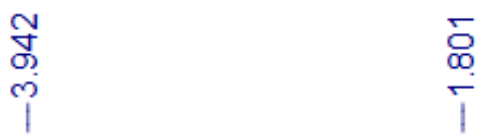
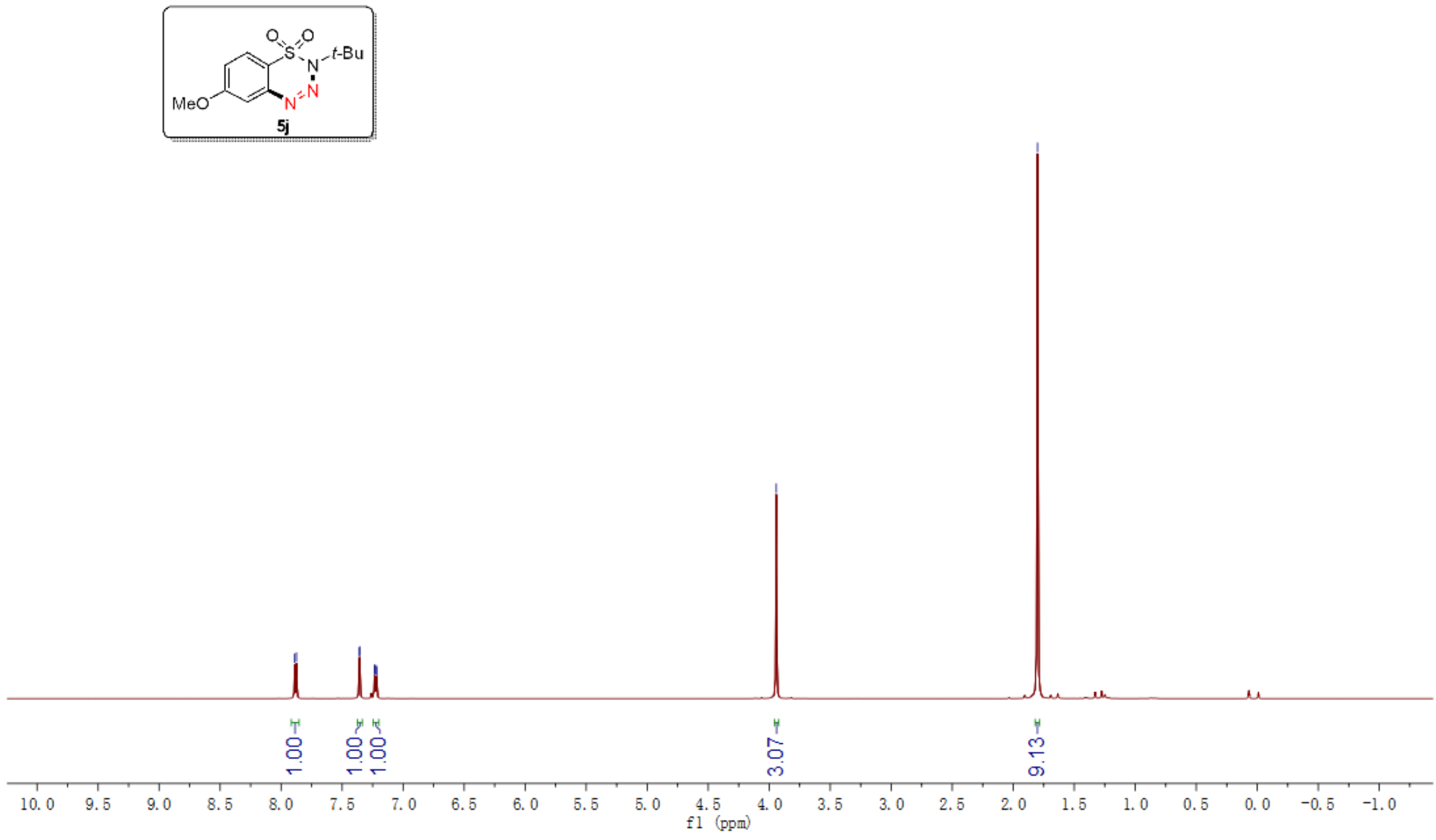
${ }^{13} \mathrm{C}$ - NMR spectrum of compound $\mathbf{5 j}\left(150 \mathrm{MHz}, \mathrm{CDCl}_{3}\right)$

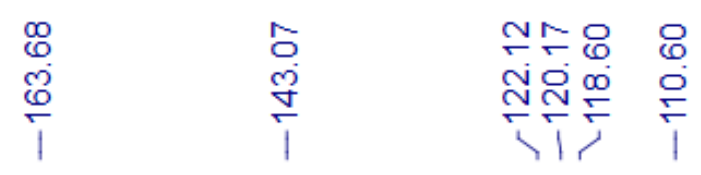

\begin{tabular}{lll}
\hline & 0 & $\infty$ \\
0 & 0 & $m$ \\
0 & 0 & 0 \\
1 & 1 & 0
\end{tabular}
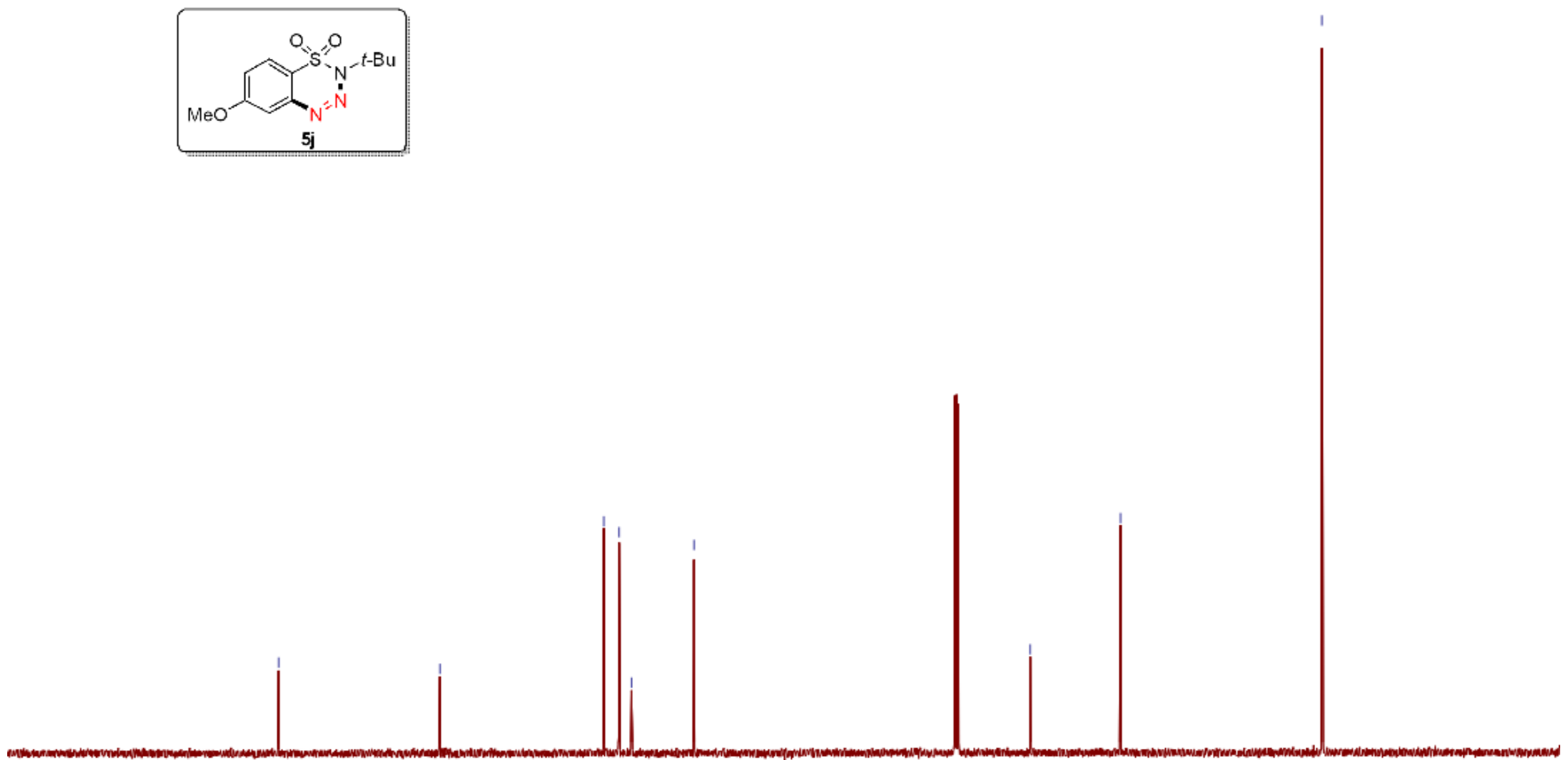
${ }^{1} \mathrm{H}$ - NMR spectrum of compound $\mathbf{5 k}\left(400 \mathrm{MHz}, \mathrm{CDCl}_{3}\right)$

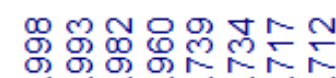

r m
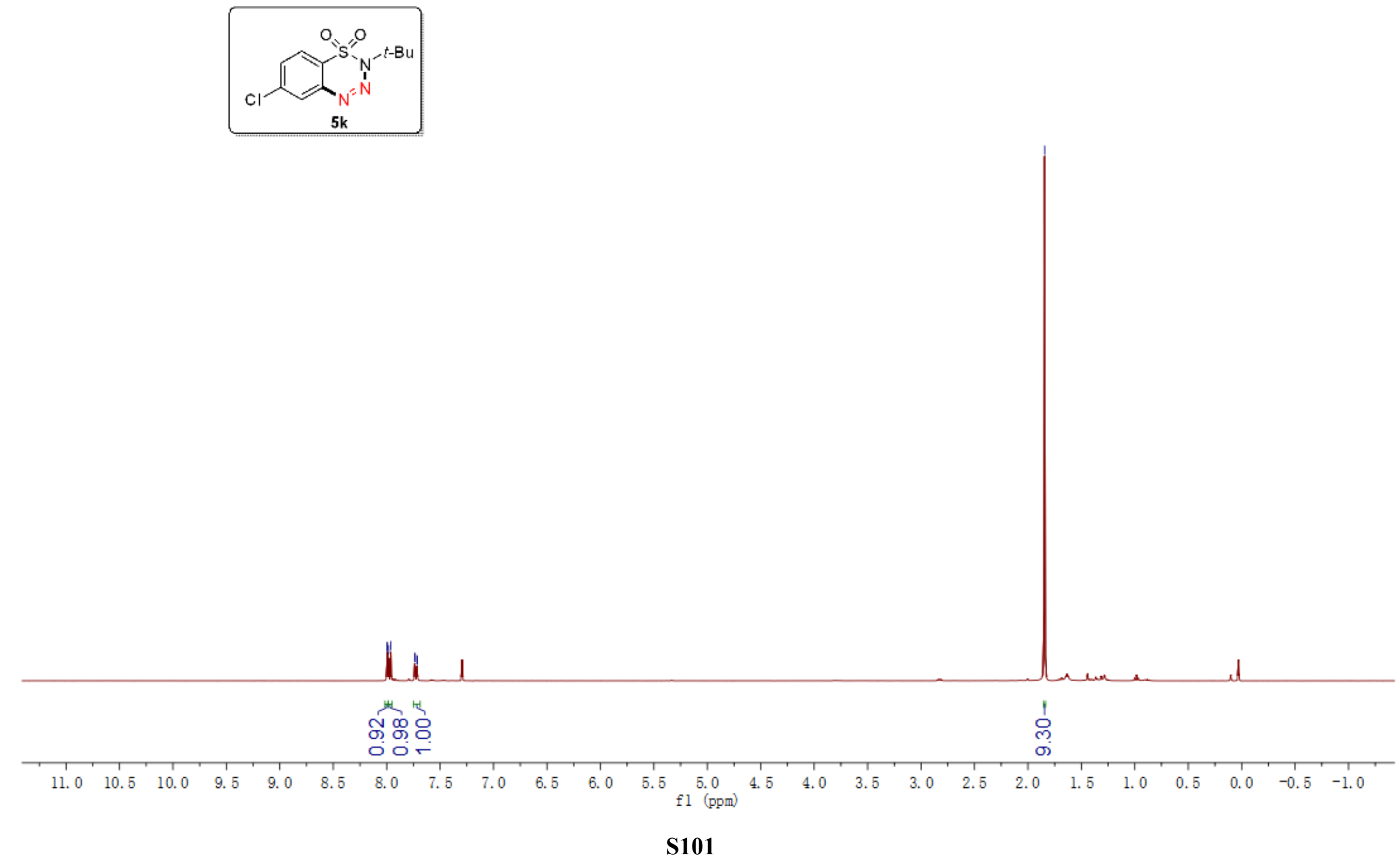
${ }^{13} \mathrm{C}$ - NMR spectrum of compound $\mathbf{5} \mathbf{k}\left(100 \mathrm{MHz}, \mathrm{CDCl}_{3}\right)$

한

(요

$\stackrel{\infty}{\infty}$

¿্ঠ

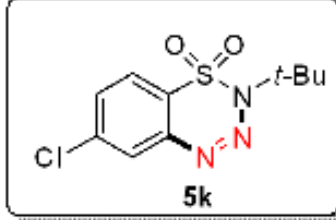

||
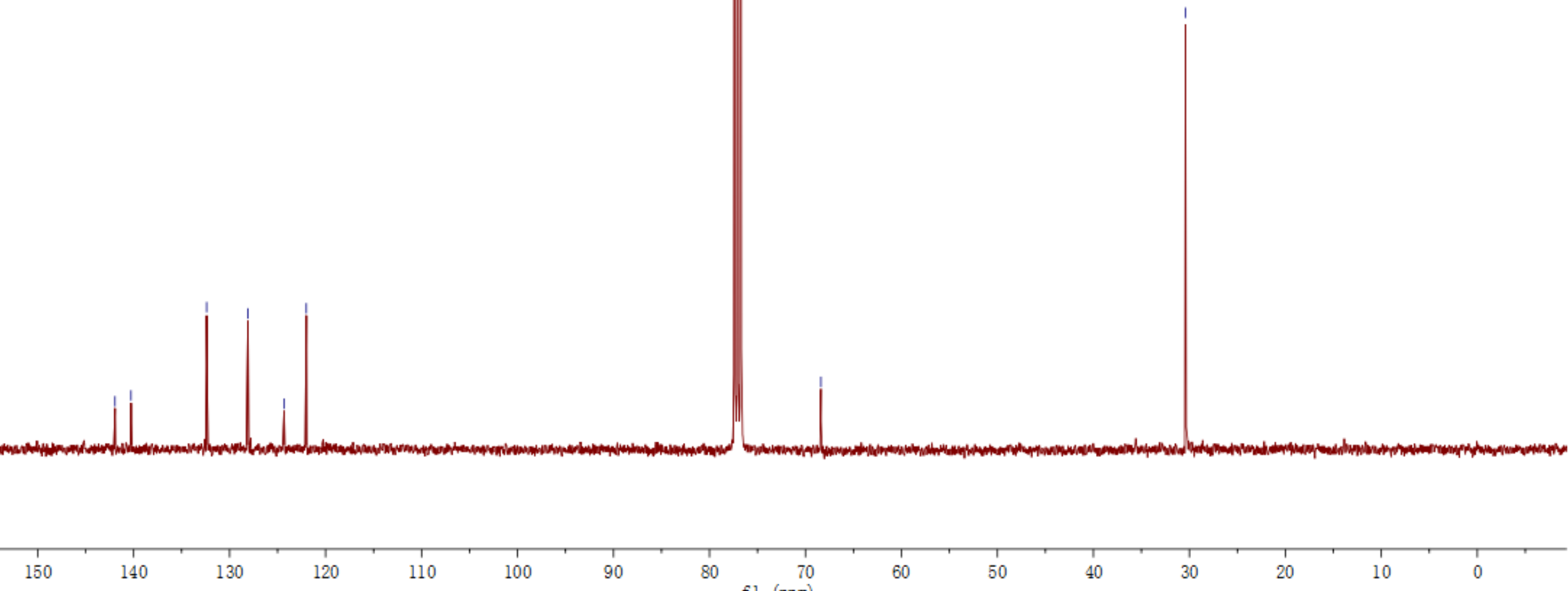

80

fl (ppm)

60

50

40

30

20

10

0 
${ }^{1} \mathrm{H}$ - NMR spectrum of compound 6 (400 MHz, DMSO- $d_{6}$ )

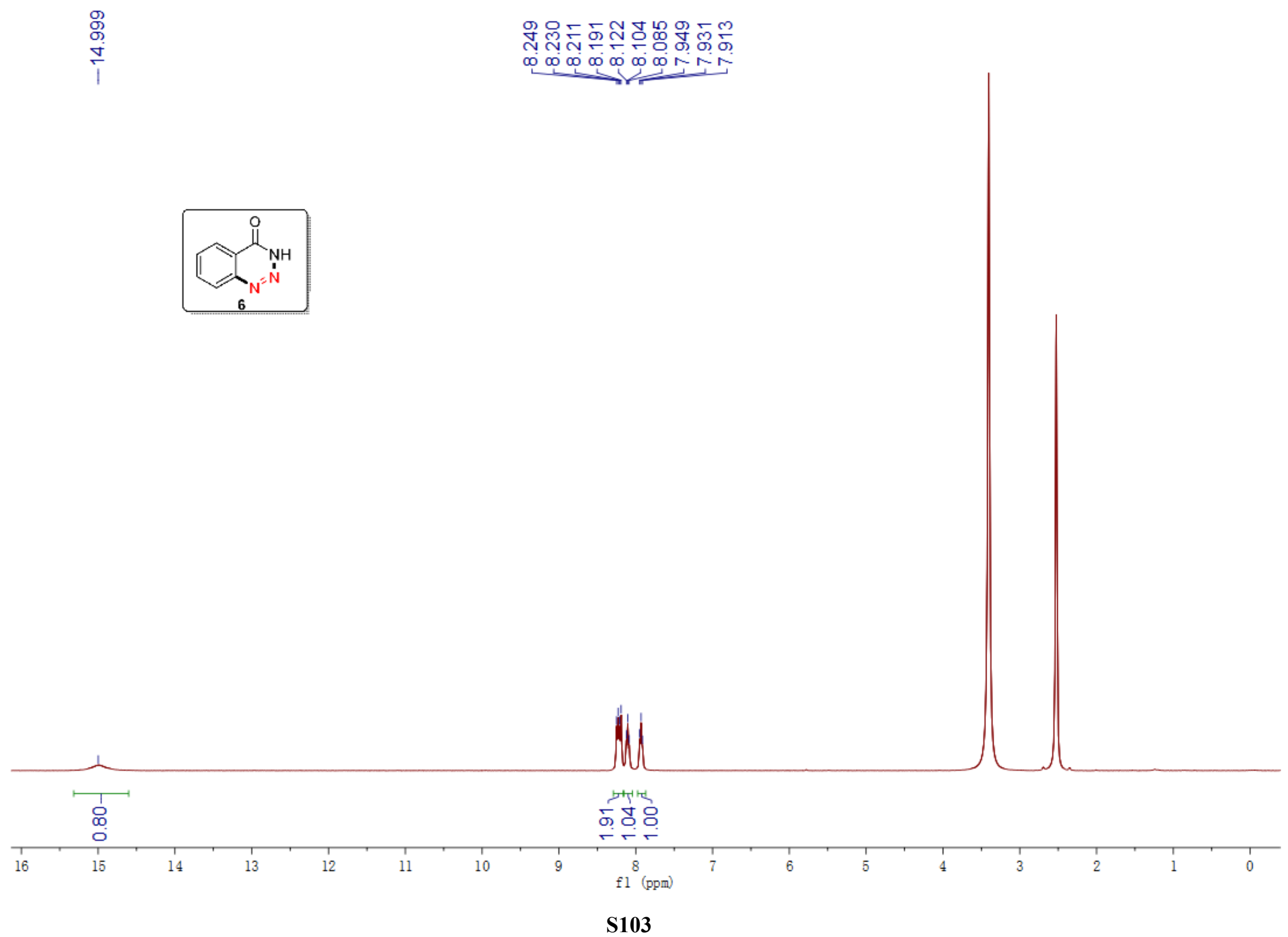


${ }^{13} \mathrm{C}$ - NMR spectrum of compound $6\left(150 \mathrm{MHz}, \mathrm{DMSO}-d_{6}\right)$

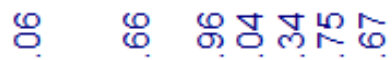

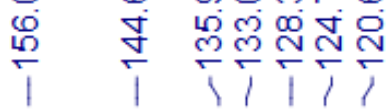
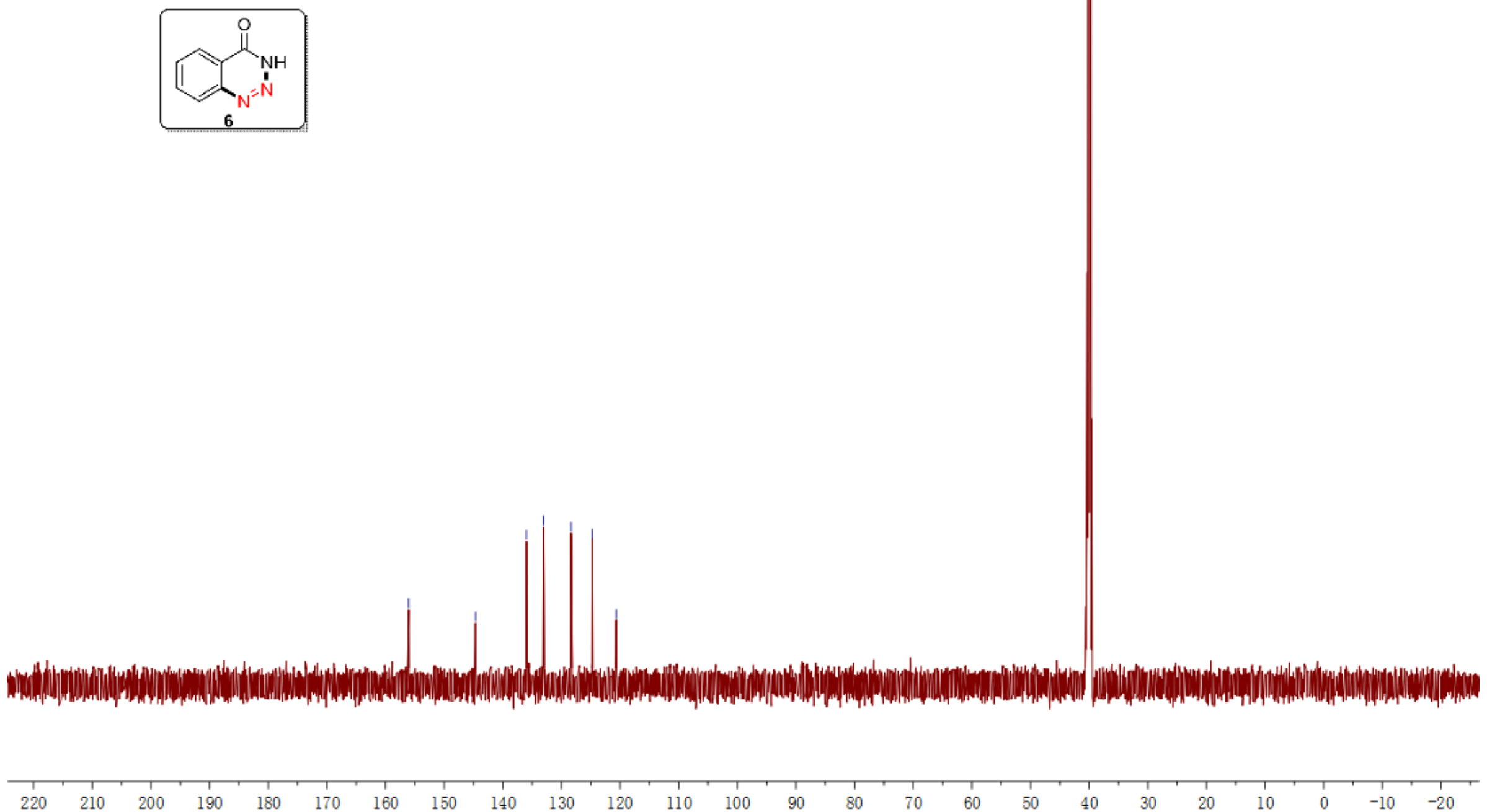
$110 \quad 100$

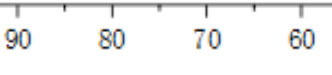


${ }^{1} \mathrm{H}$ - NMR spectrum of compound $7 \mathbf{a}\left(400 \mathrm{MHz}, \mathrm{CDCl}_{3}\right)$

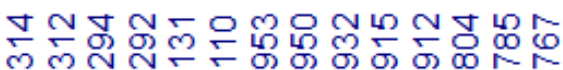

$\infty \infty \infty \infty \infty \infty \cdots$

$\underbrace{\infty}$
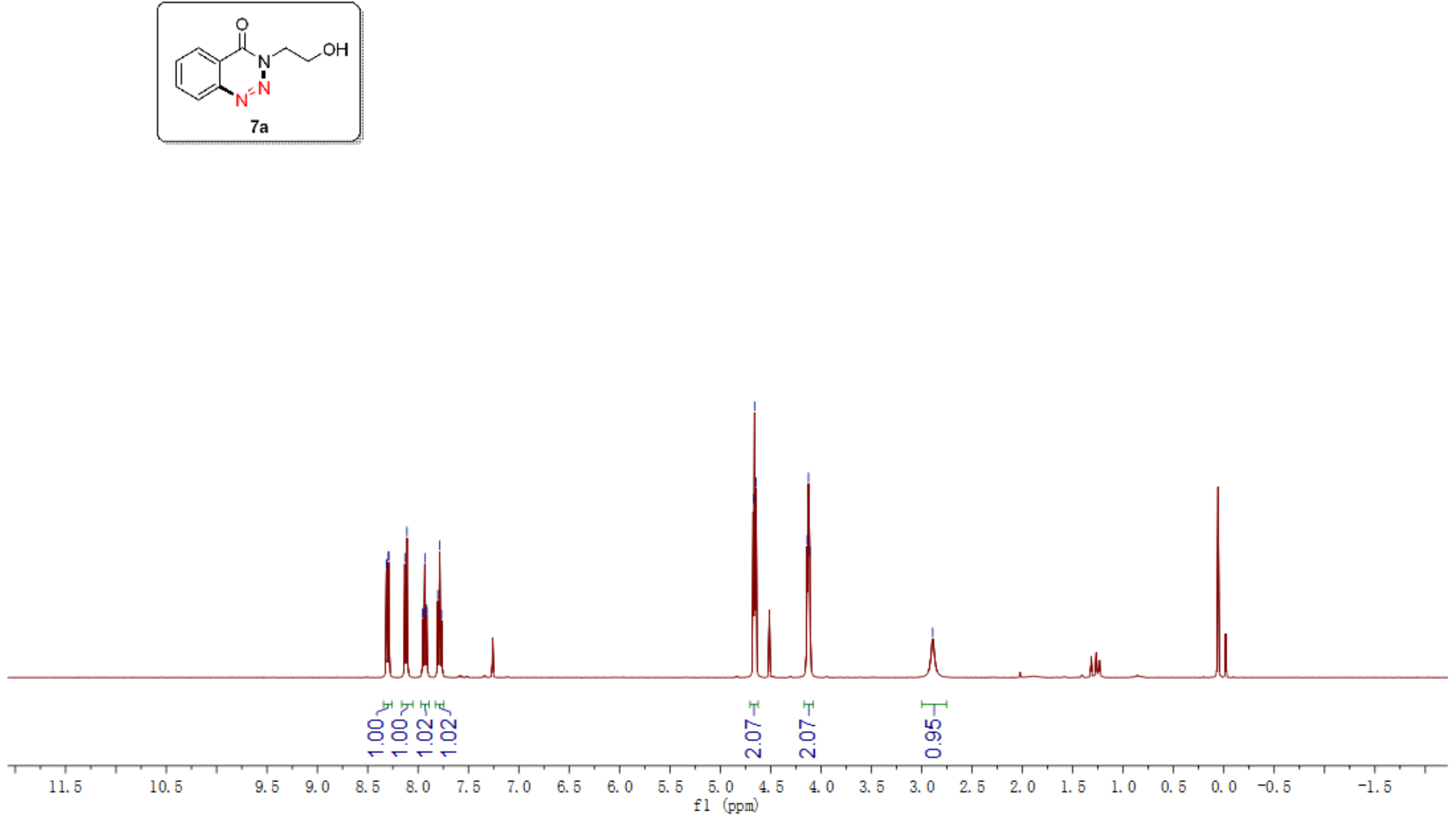
${ }^{13} \mathrm{C}$ - NMR spectrum of compound $7 \mathbf{a}\left(100 \mathrm{MHz}, \mathrm{CDCl}_{3}\right)$

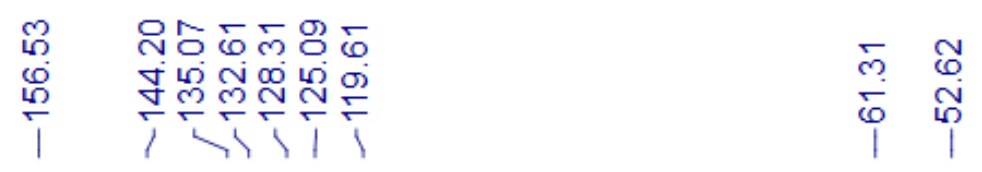
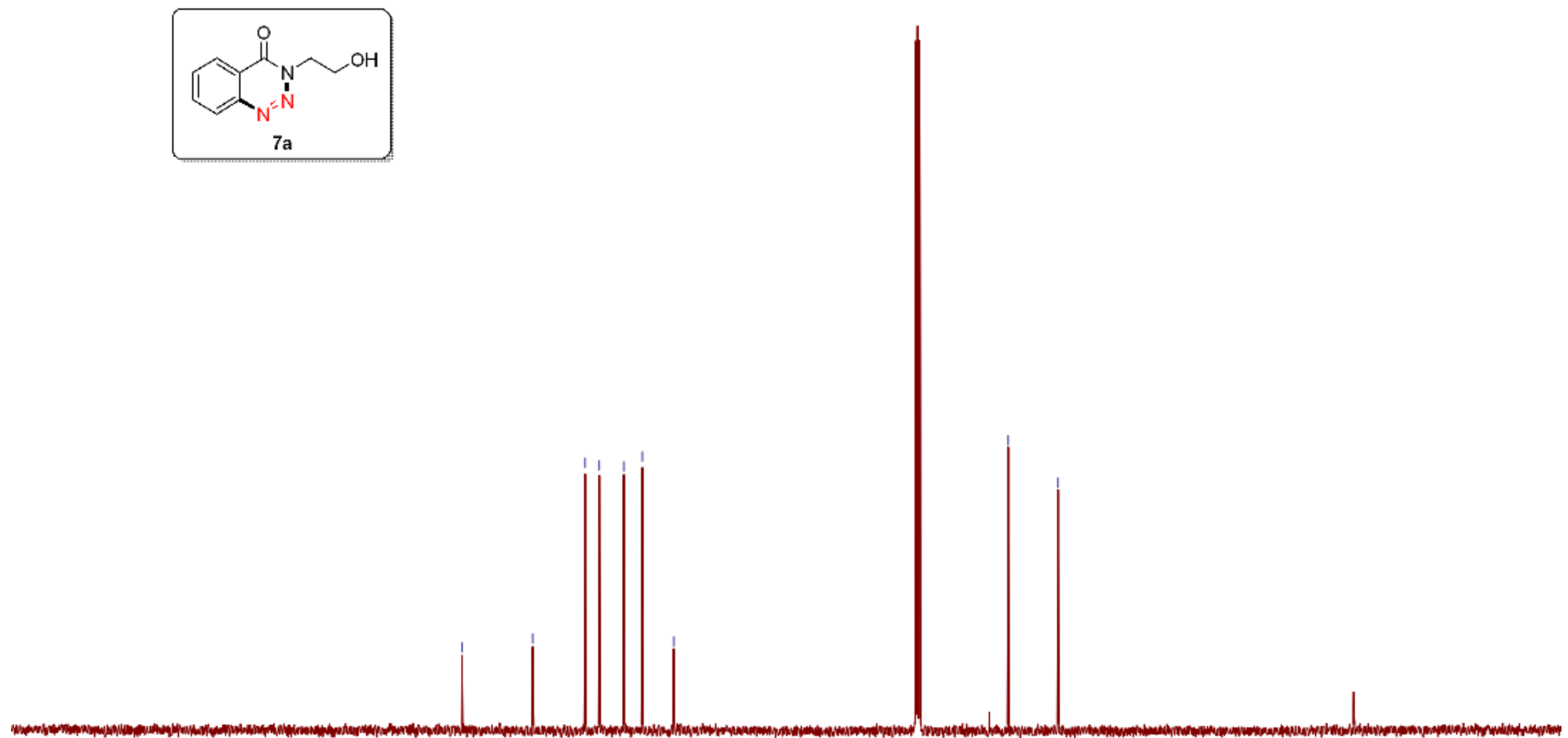

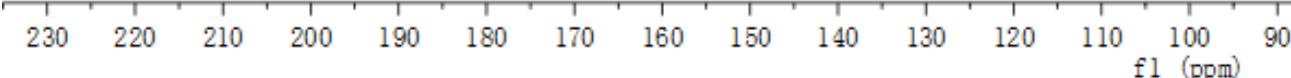

$80 \quad 70$

60

40

$30 \quad 20$

100

$\begin{array}{lll}-10 & -20 & -30\end{array}$ 
${ }^{1} \mathrm{H}$ - NMR spectrum of compound $7 \mathbf{b}\left(400 \mathrm{MHz}, \mathrm{CDCl}_{3}\right)$

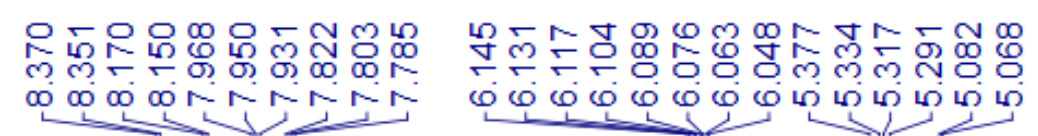
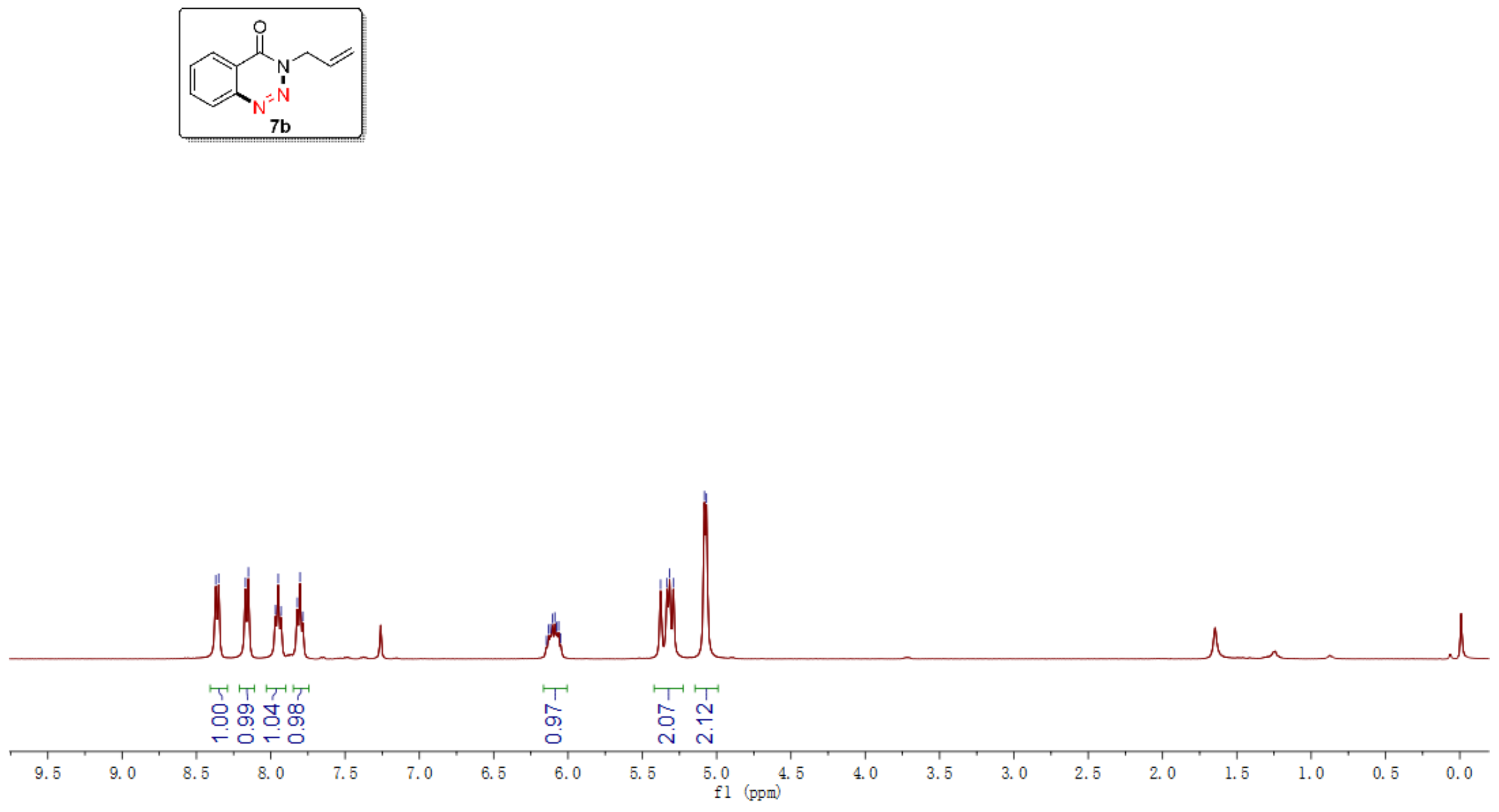
${ }^{13} \mathrm{C}-\mathrm{NMR}$ spectrum of compound $7 \mathbf{b}\left(100 \mathrm{MHz}, \mathrm{CDCl}_{3}\right)$

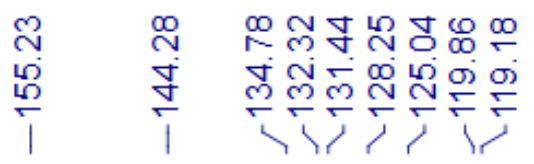

$\mathscr{8}$
No
I
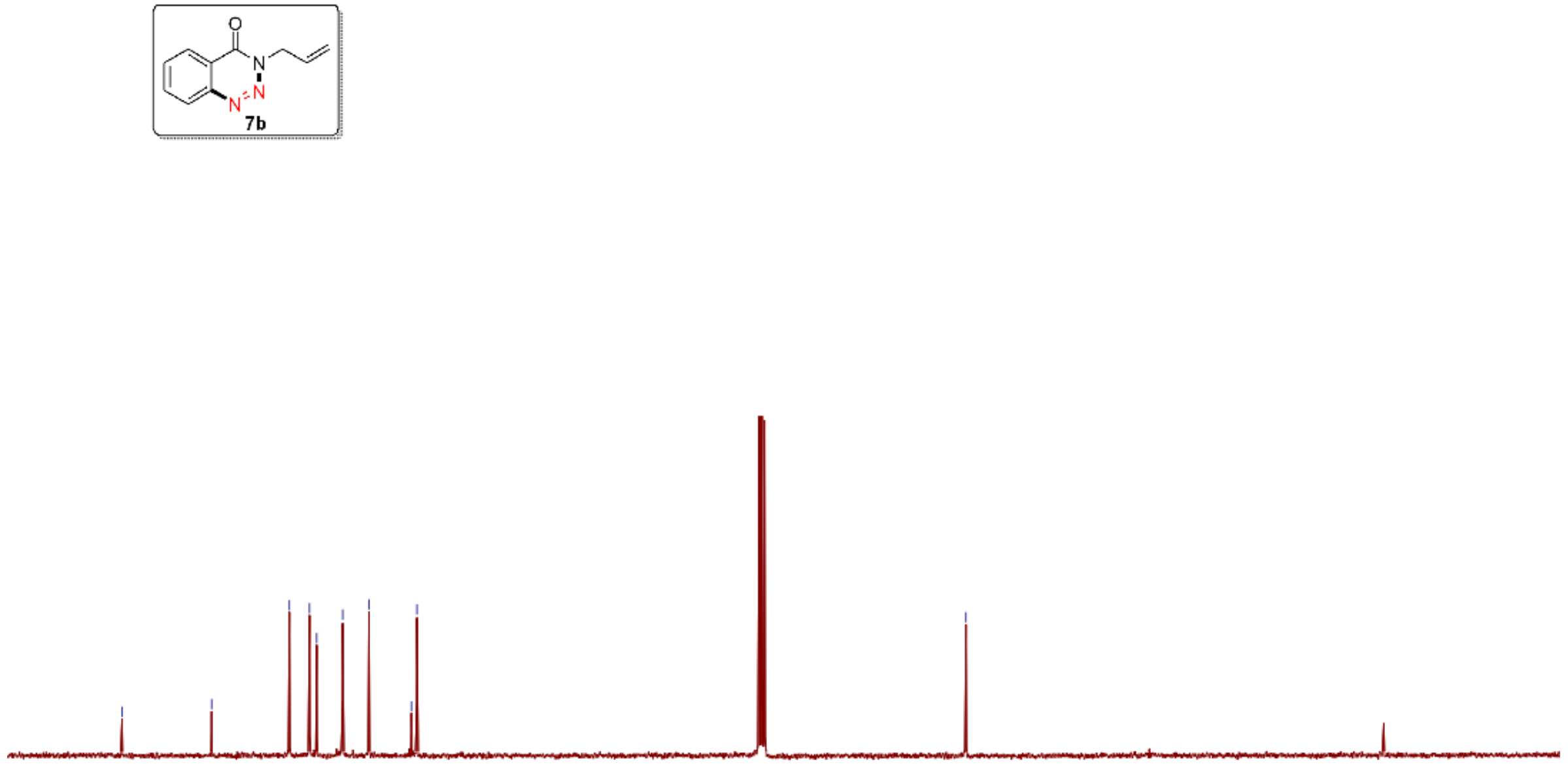
${ }^{1} \mathrm{H}$ - NMR spectrum of compound $\mathbf{7 c}\left(400 \mathrm{MHz}, \mathrm{CDCl}_{3}\right)$

๙

$\infty \infty \infty \infty \infty \cdots$

$\stackrel{\infty}{\sim} \stackrel{m}{N}$

กู่

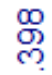

r

i
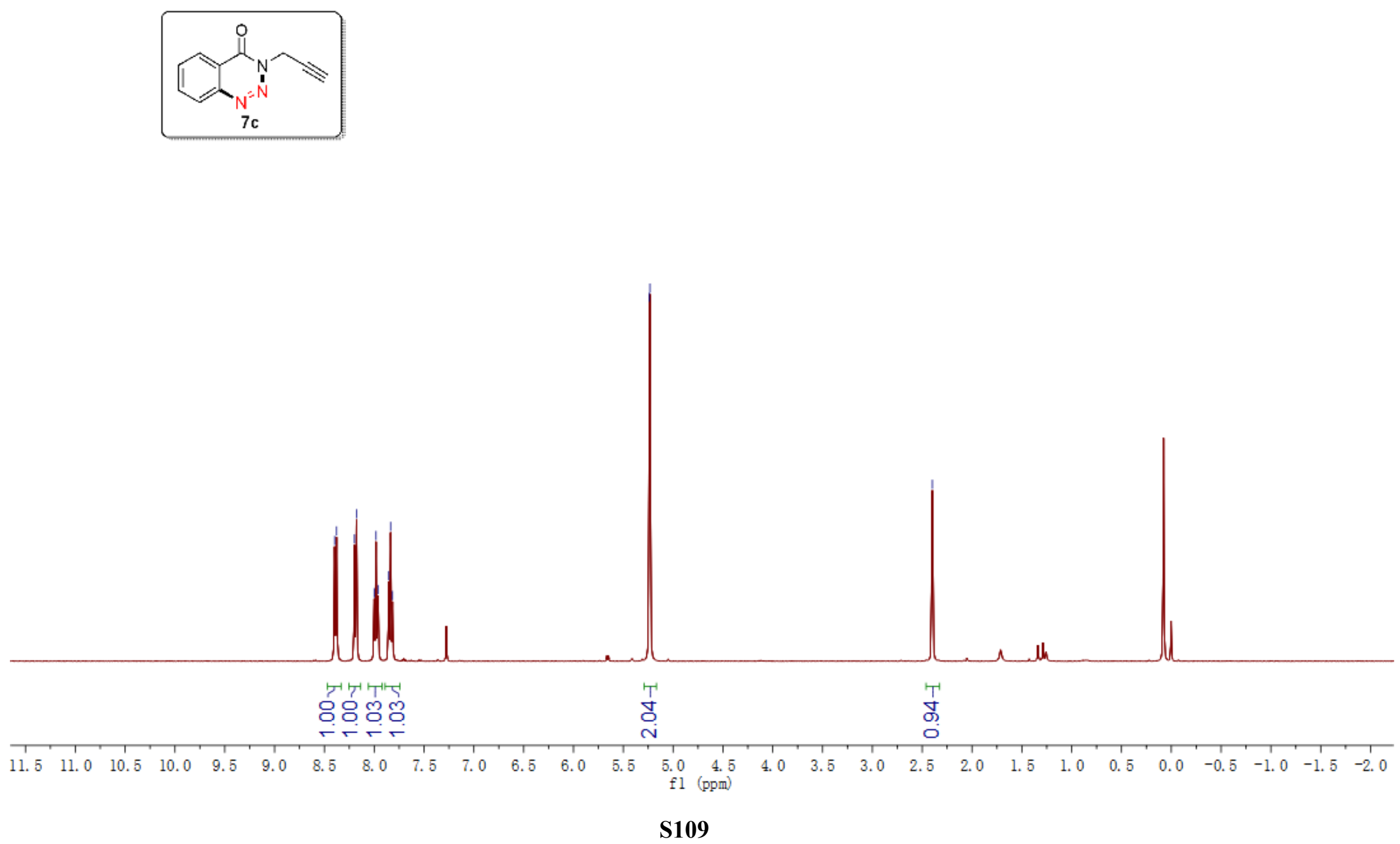
${ }^{13} \mathrm{C}$ - NMR spectrum of compound $7 \mathbf{c}\left(100 \mathrm{MHz}, \mathrm{CDCl}_{3}\right)$

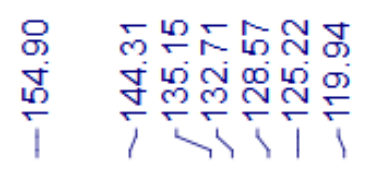

న.

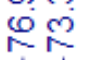

¿ั.

11

i)
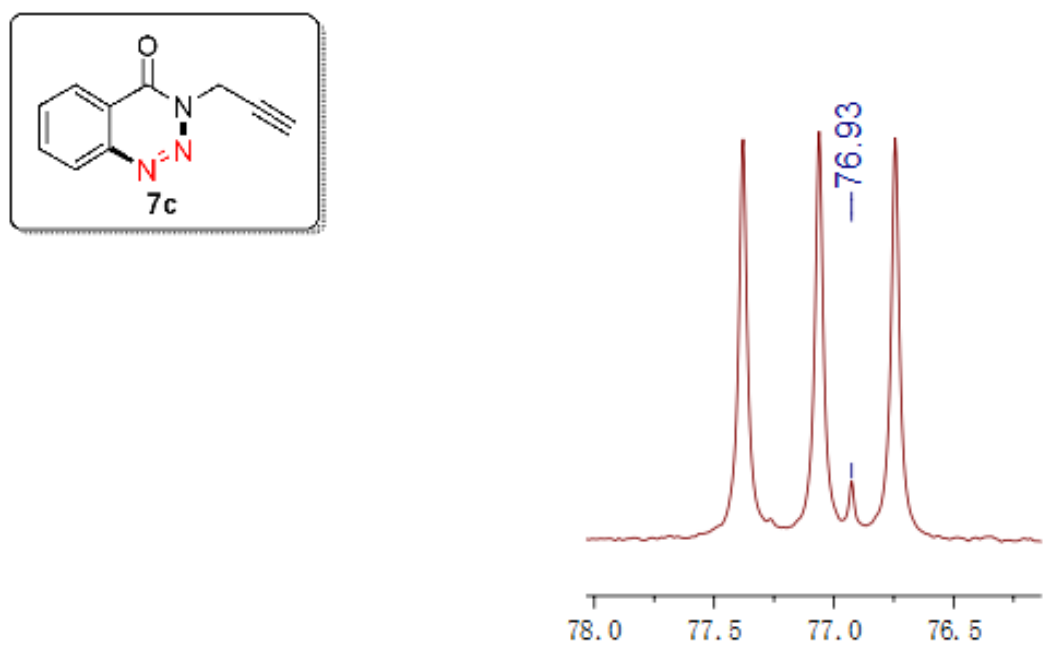

$230 \quad 220 \quad 210$ 
${ }^{1} \mathrm{H}$ - NMR spectrum of compound $\mathbf{7 d}\left(400 \mathrm{MHz}, \mathrm{CDCl}_{3}\right)$

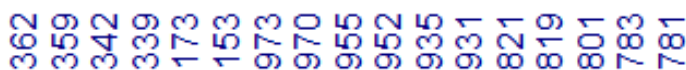

$\infty \infty \infty \infty \infty \cdots \infty$
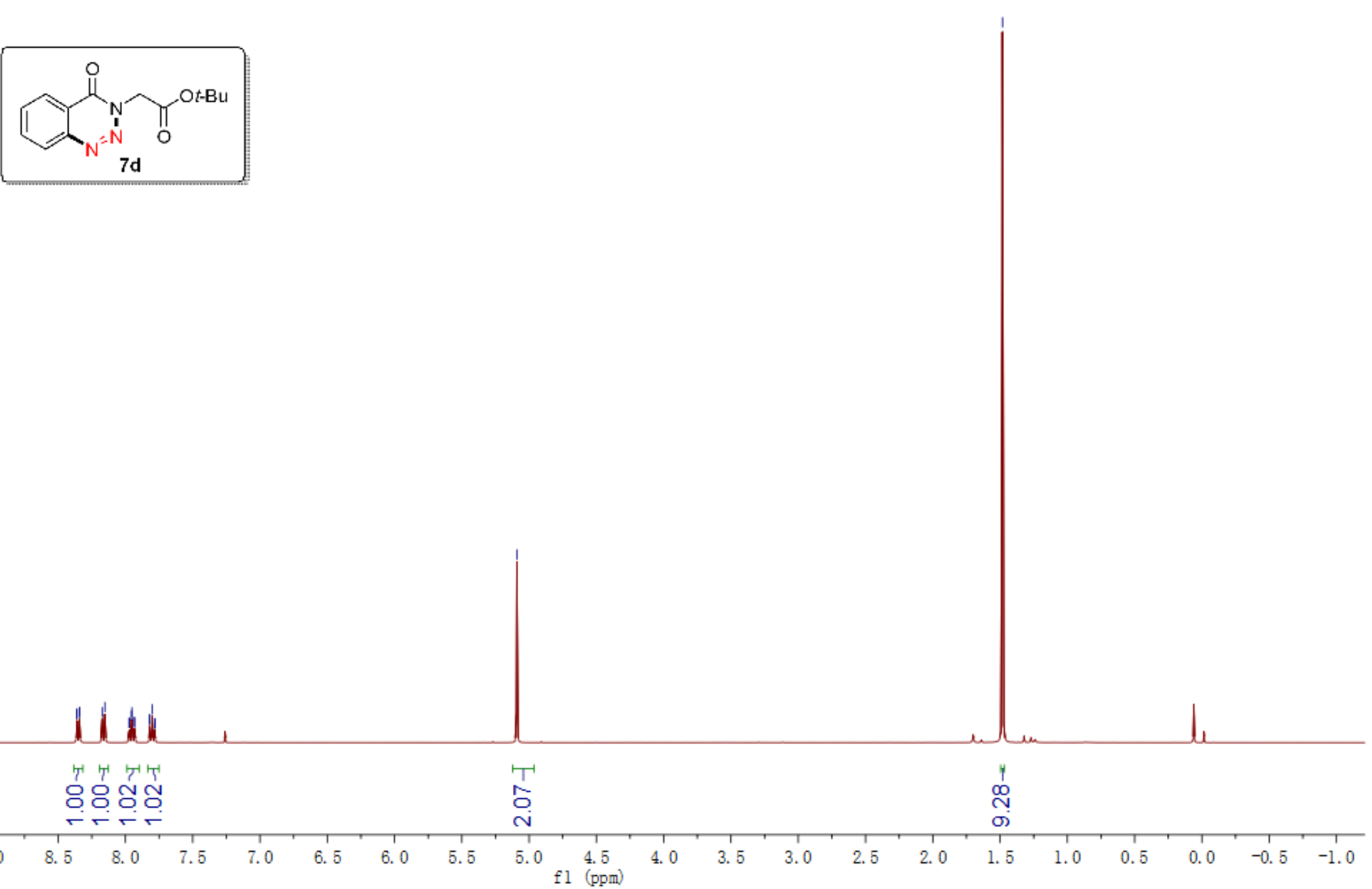
${ }^{13} \mathrm{C}$ - NMR spectrum of compound $7 \mathbf{d}\left(100 \mathrm{MHz}, \mathrm{CDCl}_{3}\right)$

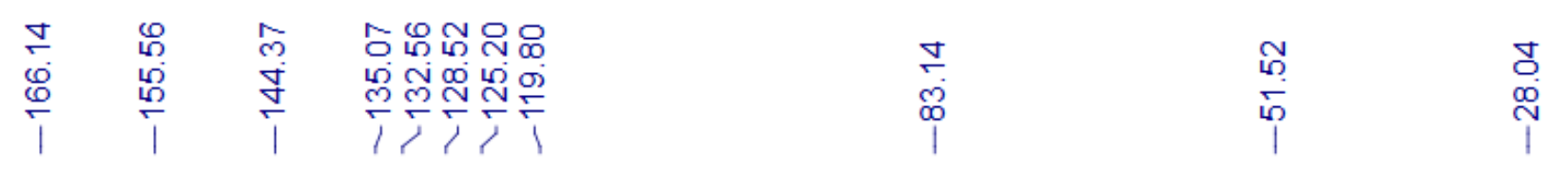
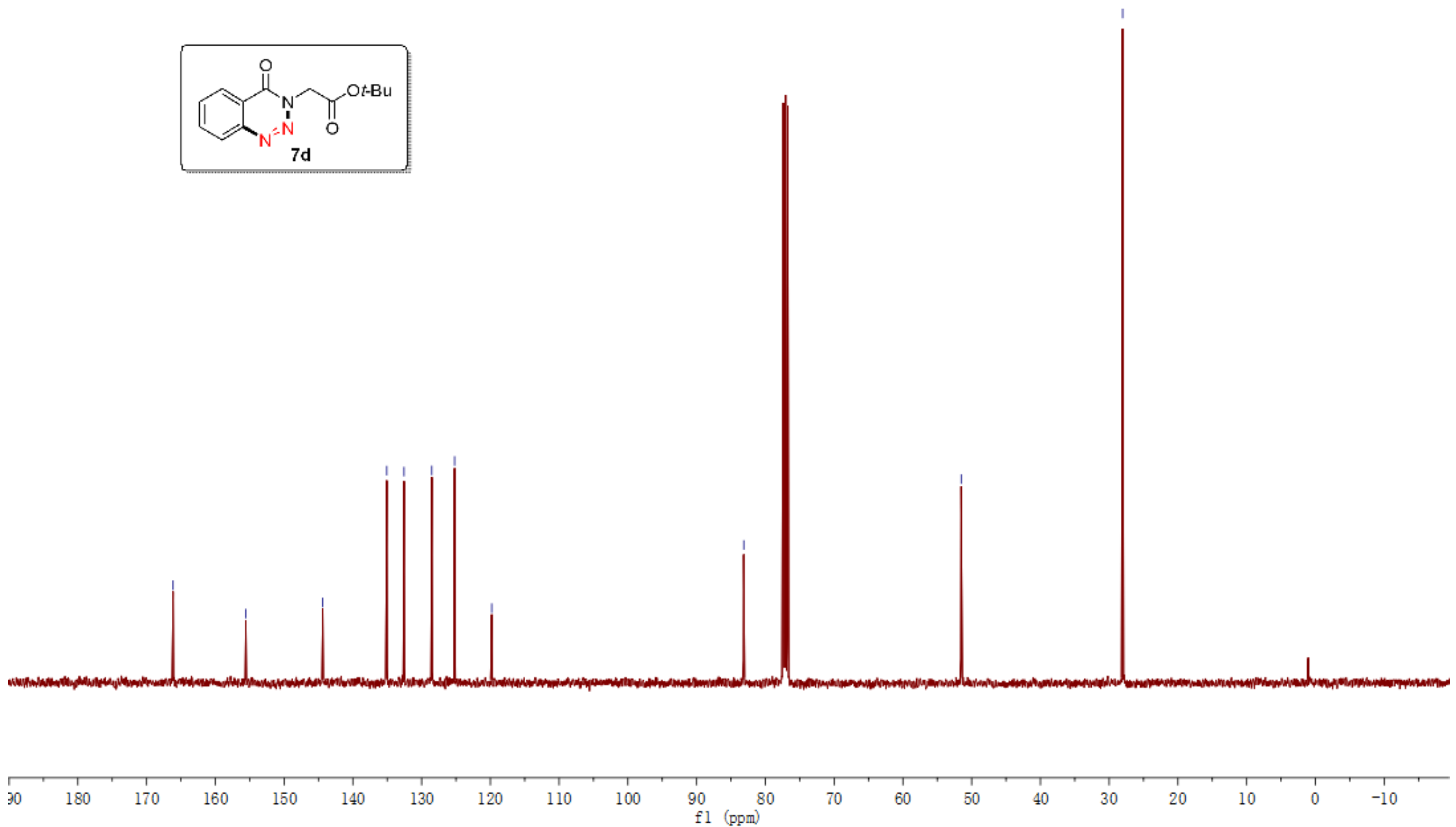
${ }^{1} \mathrm{H}$ - NMR spectrum of compound $7 \mathrm{e}\left(500 \mathrm{MHz}, \mathrm{CDCl}_{3}\right)$

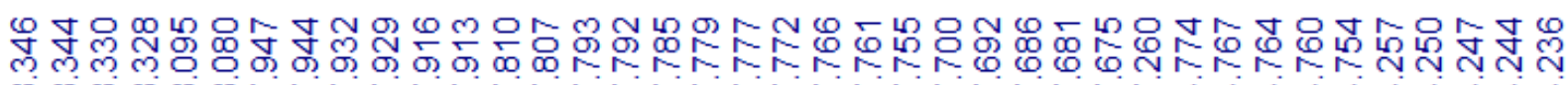

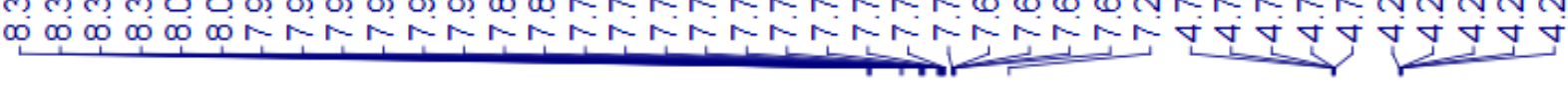
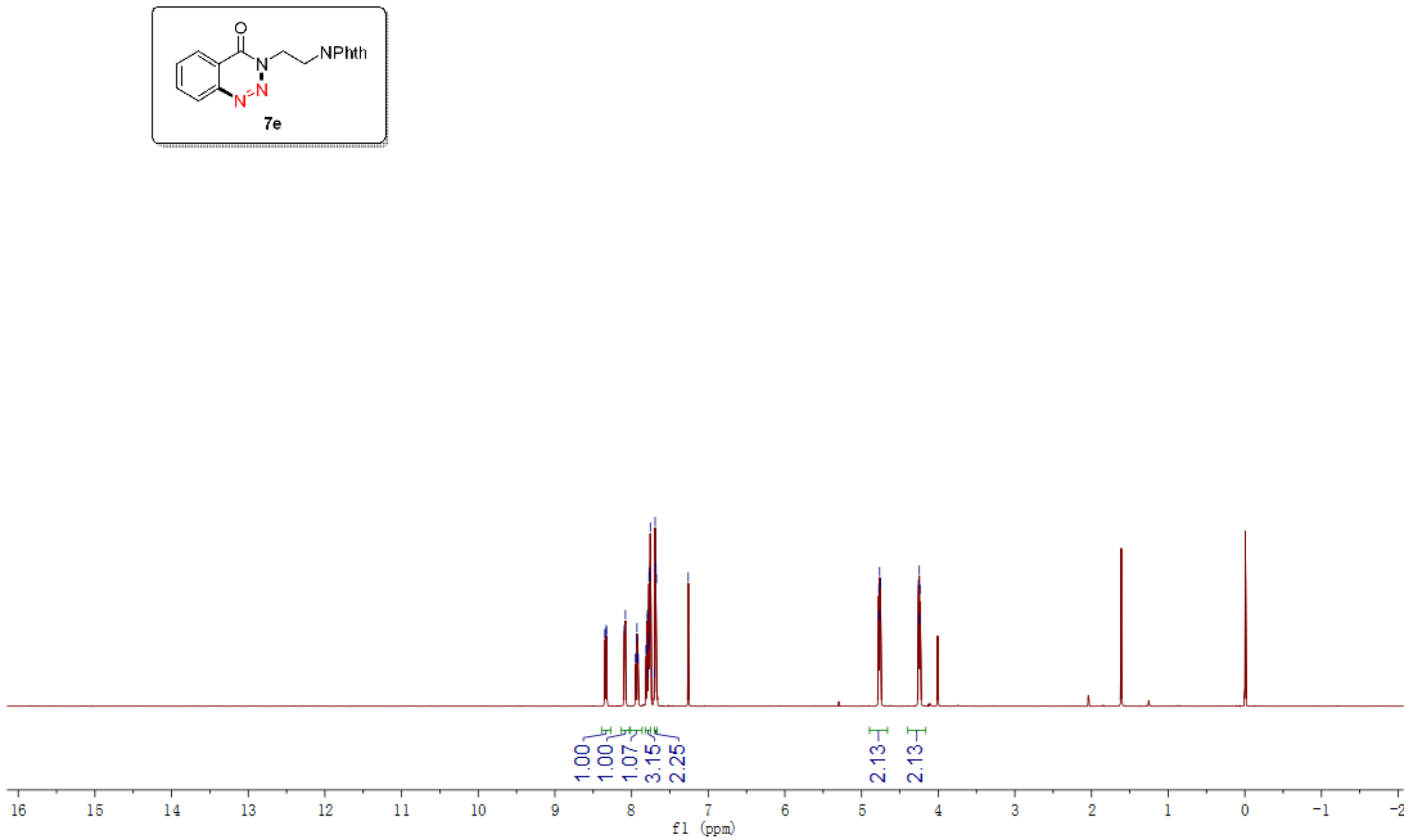
${ }^{13} \mathrm{C}$ - NMR spectrum of compound $7 \mathrm{e}\left(100 \mathrm{MHz}, \mathrm{CDCl}_{3}\right)$

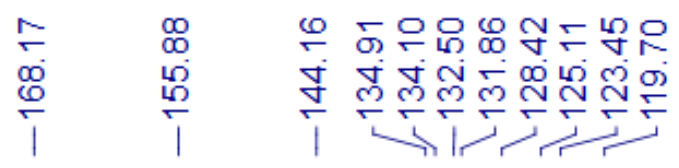

\begin{tabular}{ll}
$\mathbb{0}$ & 9 \\
0 & 0 \\
\multirow{1}{*}{} & 0
\end{tabular}
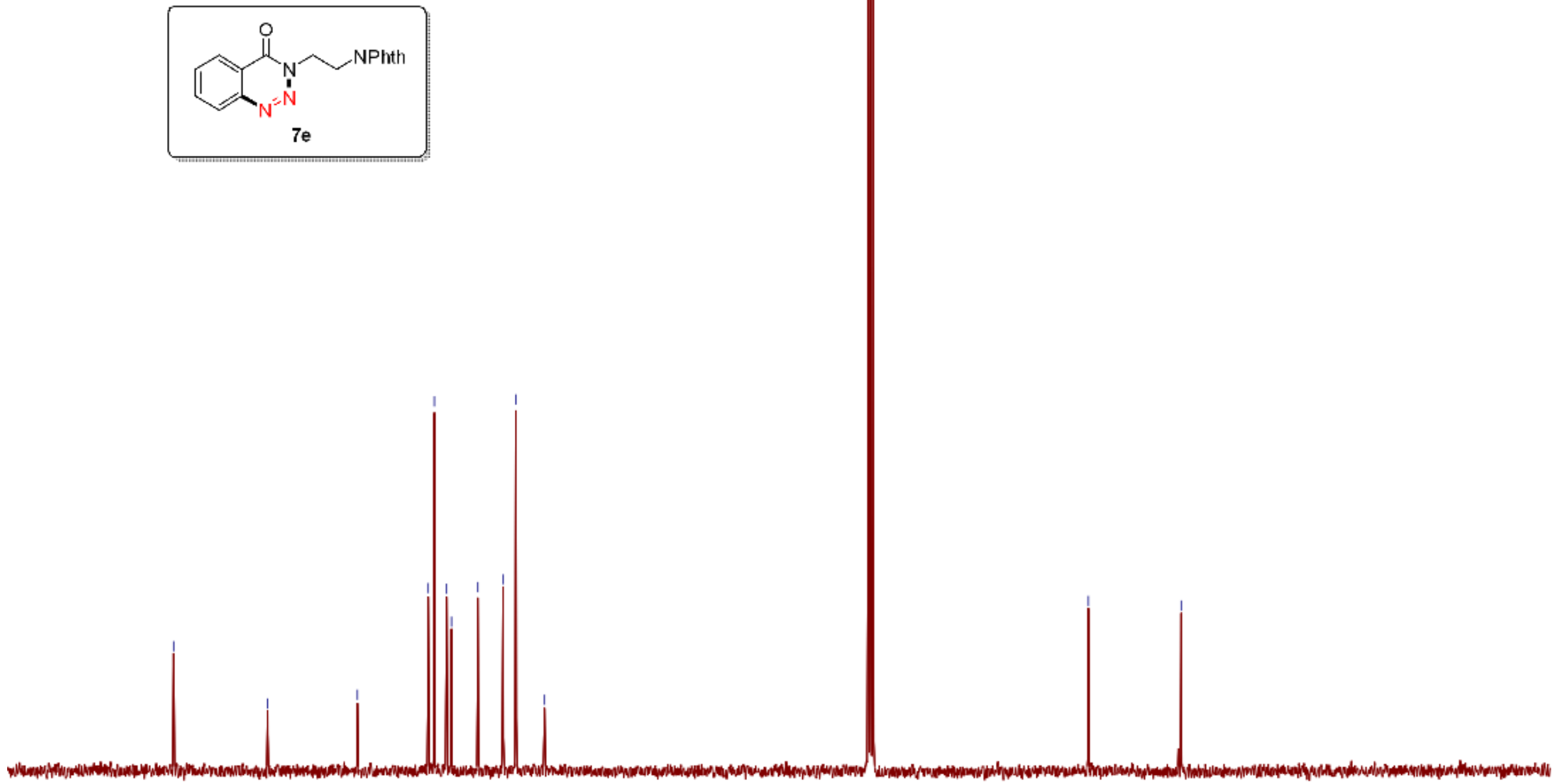

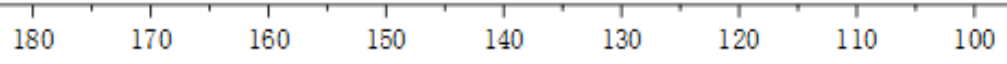
fl 190 
${ }^{1} \mathrm{H}$ - NMR spectrum of compound $9\left(500 \mathrm{MHz}, \mathrm{CDCl}_{3}\right)$
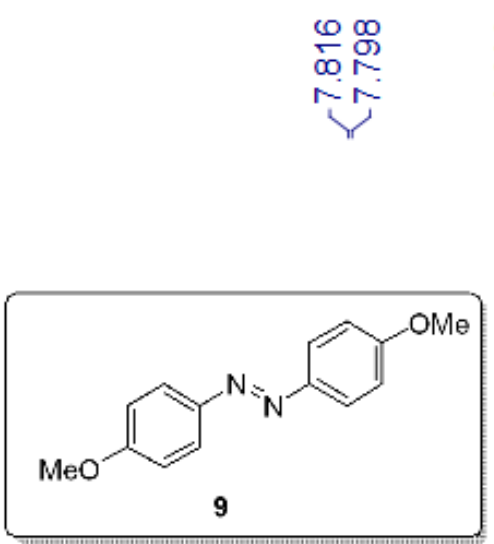

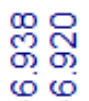

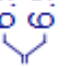

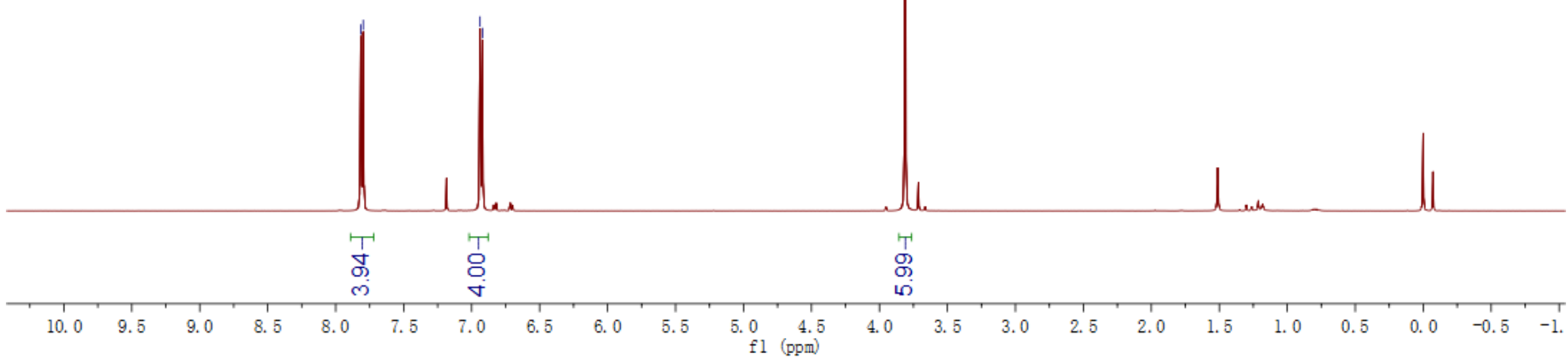


${ }^{13} \mathrm{C}$ - NMR spectrum of compound $9\left(125 \mathrm{MHz}, \mathrm{CDCl}_{3}\right)$

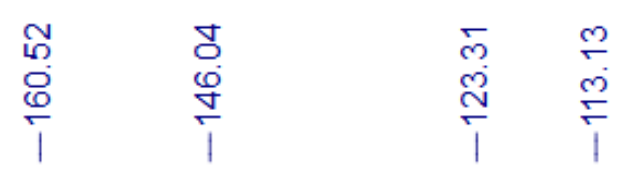


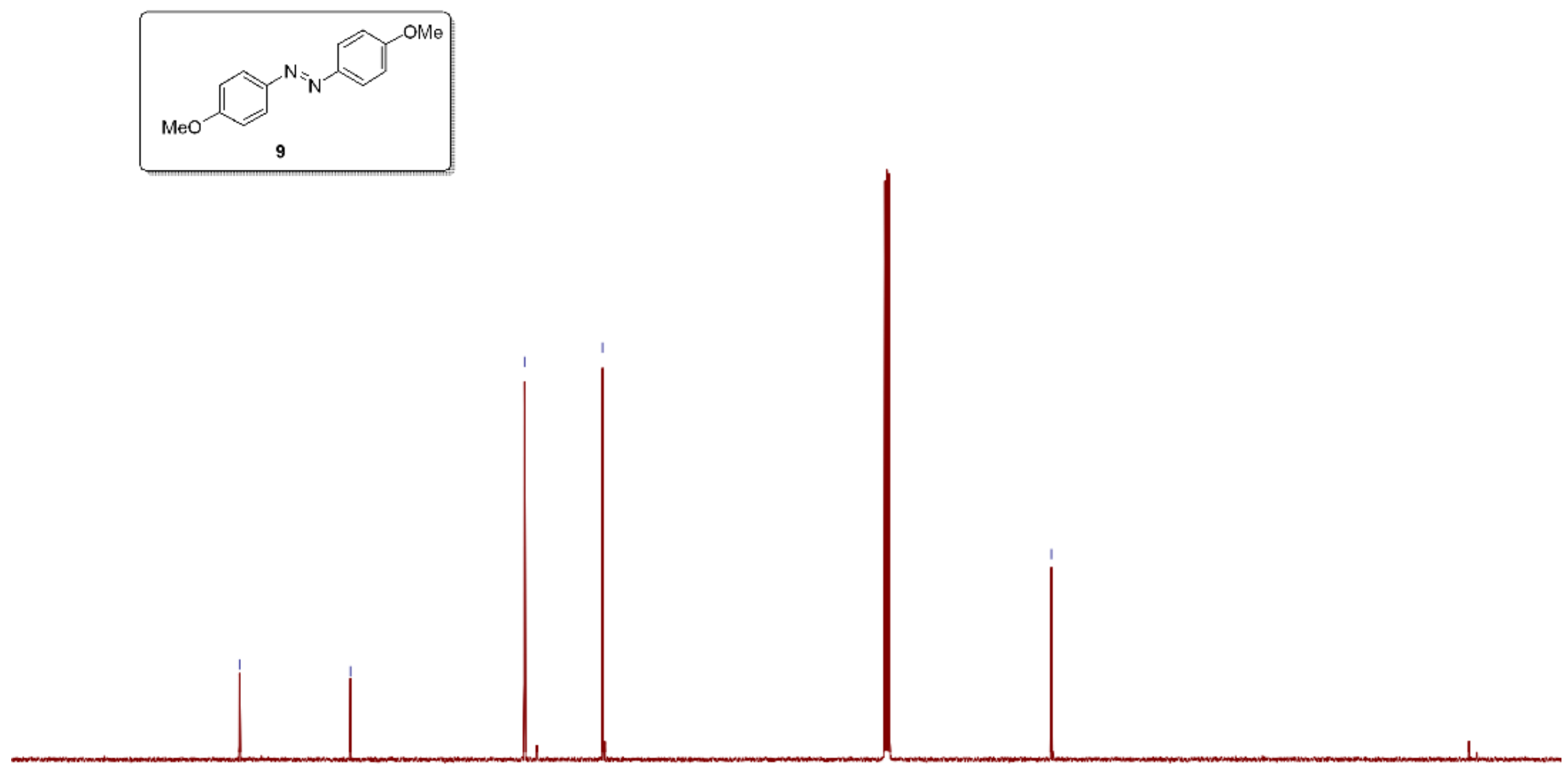

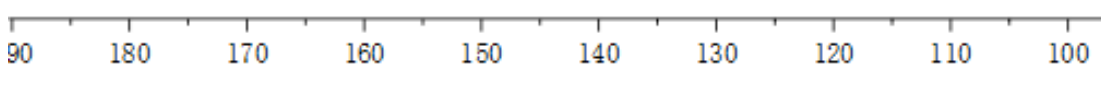

fl 190

$80 \quad 70$

60

50

40

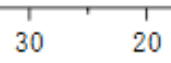

$20 \quad 10 \quad 10 \quad-10$ 